\title{
Development and Validation of a Standard Test Method For Sequential Batch Extraction of Waste With Acidic Extraction Fluid
}

\section{Topical Report}

\section{S.S. Sorini}

August 1993

Work Performed Under Contract No.: De-FC21-86MC11076

For

U.S. Department of Energy

Office of Fossil Energy

Morgantown Energy Technology Center

Morgantown, West Virginia

By

The University of Wyoming Research Corporation

Western Research Institute

Laramie, Wyoming 


\section{DISCLAIMER}

This report was prepared as an account of work sponsored by an agency of the United States Government. Neither the United States Government nor any agency thereof, nor any of their employees, makes any warranty, express or implied, or assumes any legal liability or responsibility for the accuracy, completeness, or usefulness of any information, apparatus, product, or process disclosed, or represents that its use would not infringe privately owned rights. Reference herein to any specific commercial product, process, or service by trade name, trademark, manufacturer, or otherwise does not necessarily constitute or imply its endorsement, recommendation, or favoring by the United States Government or any agency thereof. The views and opinions of authors expressed herein do not necessarily state or reflect those of the United States Government or any agency thereof.

This report has been reproduced directly from the best available copy.

Available to DOE and DOE contractors from the Office of Scientific and Technical Information, P.O. Box 62, Oak Ridge, TN 37831; prices available at (615) 576-8401.

Available to the public from the National Technical Information Service, U.S. Department of Commerce, 5285 Port Royal Rd., Springfield, VA 22161; phone orders accepted at (703) 487-4650. 


\section{DISCLAIMER}

Portions of this document may be illegible in electronic image products. Images are produced from the best available original document. 
Development and Validation of a Standard Test Method For Sequential Batch Extraction of Waste With Acidic Extraction Fluid

\author{
Topical Report
}

\author{
S.S. Sorini
}

Work Performed Under Contract No.: De-FC21-86MC11076

\author{
For \\ U.S. Department of Energy \\ Office of Fossil Energy \\ Morgantown Energy Technology Center \\ P.O. Box 880 \\ Morgantown, West Virginia 26507-0880
}

\author{
By \\ The University of Wyoming Research Corporation \\ Western Research Institute \\ P.O. Box 3395 \\ Laramie, Wyoming 82071
}

August 1993 
LIST OF TABLES.......................................

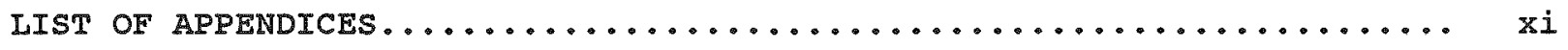

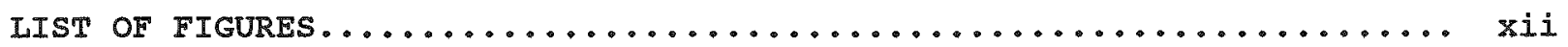

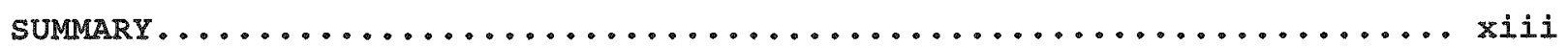

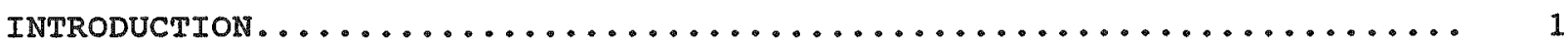

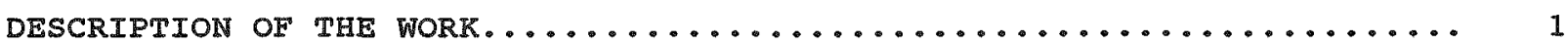

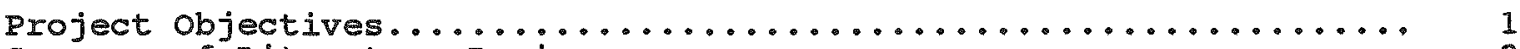

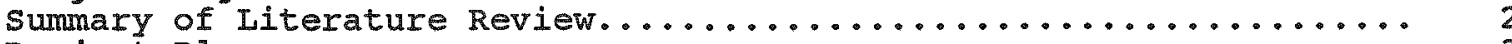

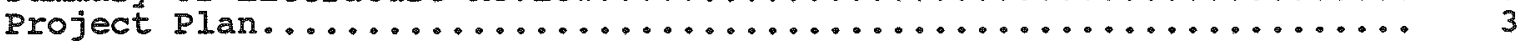

Development of the Sequential Batch Extraction Method Using

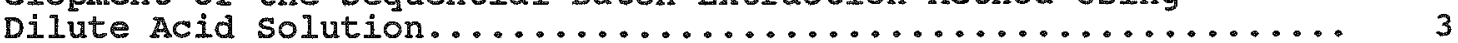

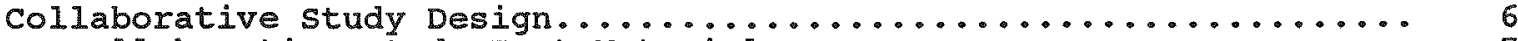

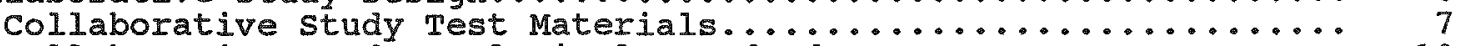

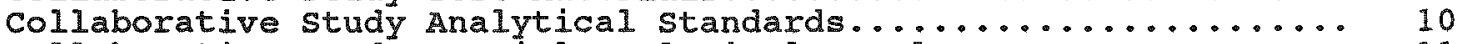

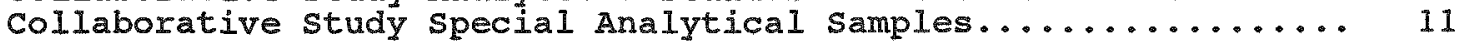

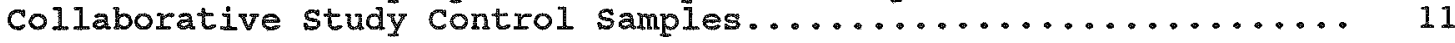

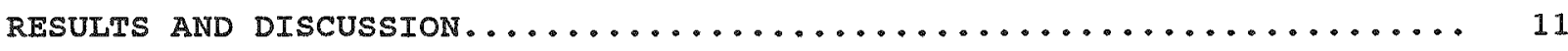

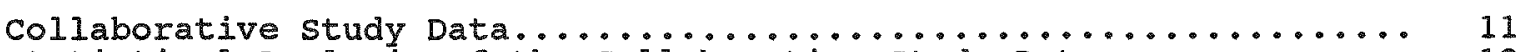

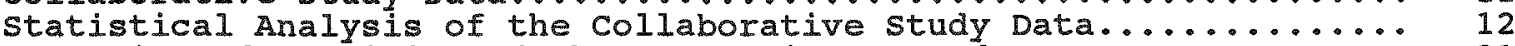

Estimated Precision of the Extraction Procedure.............. 31

Evaluation of the Data from Analysis of the special

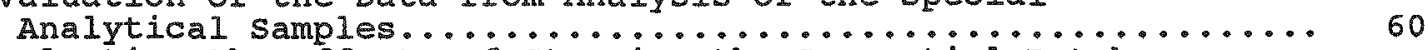

Evaluating the Effects of stopping the sequential Batch

Extraction Method over the Weekend..................... 65

Evaluation of the Control Data Versus Acidic Extraction

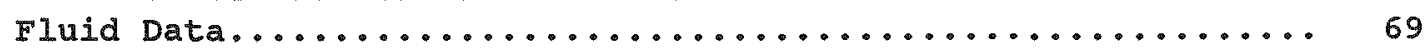

Analytical Bias Evaluations............................ 71

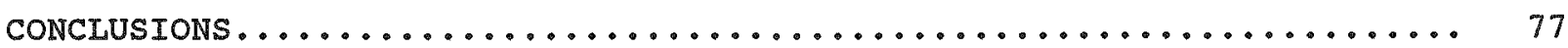

Estimated Precision of the Extraction Procedure................ 77

Effect of Filter Pore Size and Digestion Versus Nondigestion

on the Elemental Concentrations Determined in the

sequential Batch Extracts............................

Effect of stopping the sequential Batch Extraction Method

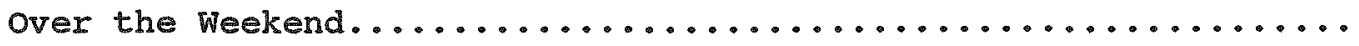

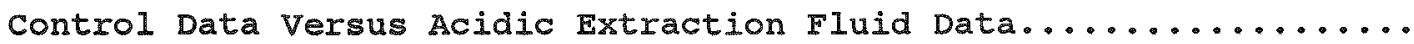

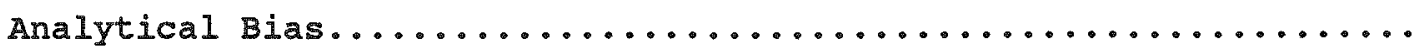

PRECISION AND BIAS STATEMENTS TO BE ADDED TO ASTM METHOD D5284-92..... 79

RECOMMENDATIONS .....................................

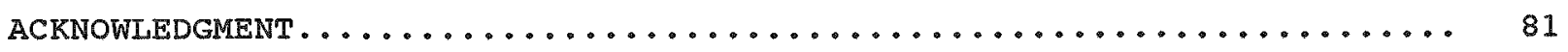

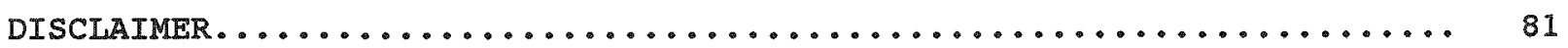

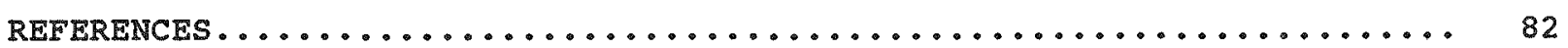




\section{LIST OF TABLES}

Table

1. Percent solid in the spray Dryer Waste and the composite

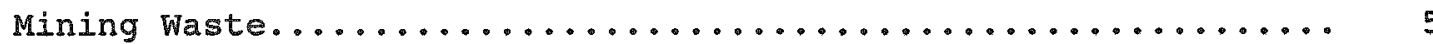

2. Elemental Concentration Ranges in the spray Dryer

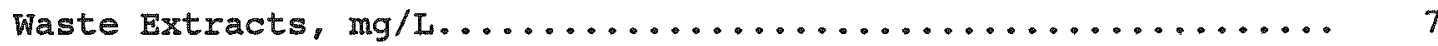

3. Elemental Concentration Ranges in the Composite Mining

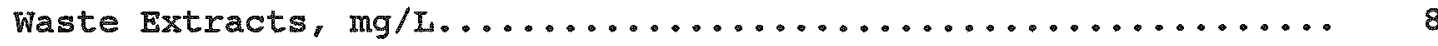

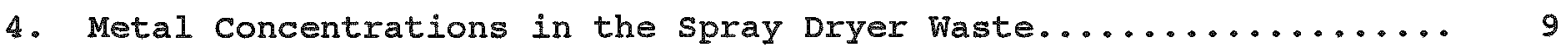

5. Spray Dryer Waste Homogeneity..............................

6. Metal Concentrations in the Composite Mining Waste............. 9

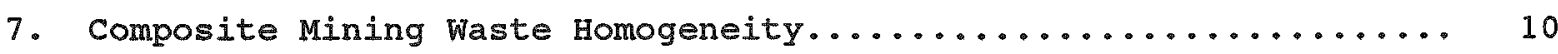

8. Elemental Concentrations in the spray Dryer waste

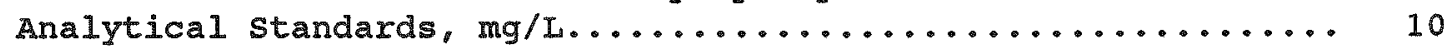

9. Elemental Concentrations in the Composite Mining Waste Analytical standards, $\operatorname{mg} /$ L....................... 10

10. Final statistics for Aluminum in the spray Dryer waste sequential Batch Extracts, $\mu g / g \ldots \ldots \ldots \ldots \ldots \ldots \ldots \ldots \ldots$

11. Final statistics for Barium in the spray Dryer Waste

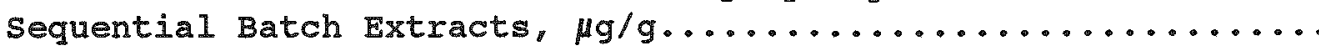

12. Final statistics for Boron in the spray Dryer waste

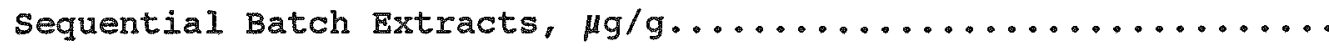

13. Final statistics for Calcium in the spray Dryer Waste sequential Batch Extracts, $\mu g / g \ldots \ldots \ldots \ldots \ldots \ldots \ldots \ldots \ldots \ldots \ldots . . . \ldots$

14. Final statistics for chromium in the spray Dryer waste Sequential Batch Extracts, $\mu g / g \ldots \ldots \ldots \ldots \ldots \ldots \ldots \ldots \ldots \ldots \ldots \ldots . . \ldots \ldots$

15. Final statistics for silicon in the spray Dryer Waste

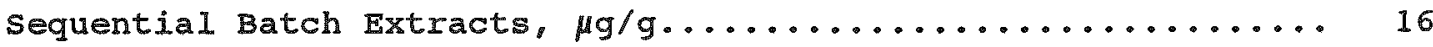

16. Final statistics for sodium in the spray Dryer waste

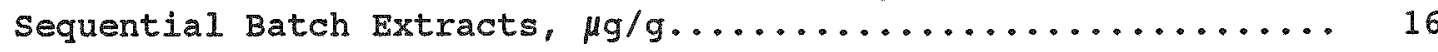

17. Final statistics for strontium in the spray Dryer waste

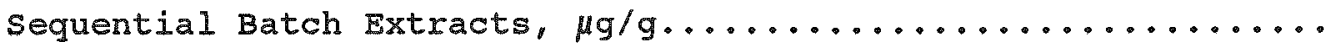




\section{IIST OF TABLES (continued)}

18. Final statistics for Barium in the composite Mining waste

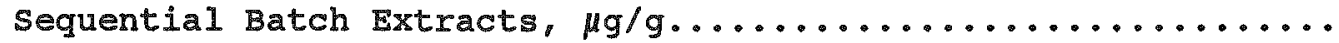

19. Final statistics for Calcium in the composite Mining Waste

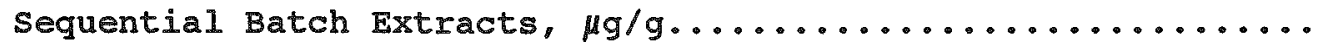

20. Final statistics for Lead in the composite Mining Waste

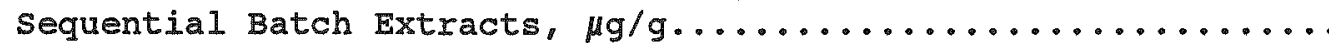

21. Final statistics for Magnesium in the Composite Mining waste

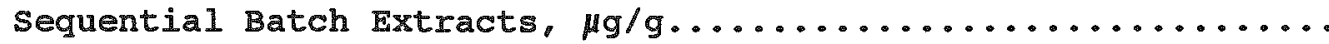

22. Final statistics for Manganese in the Composite Mining Waste Sequential Batch Extracts, $\mu g / g \ldots \ldots \ldots \ldots \ldots \ldots \ldots \ldots \ldots \ldots \ldots \ldots$

23. Final statistics for silicon in the Composite Mining Waste Sequential Batch Extracts, $\mu g / g \ldots \ldots \ldots \ldots \ldots \ldots \ldots \ldots \ldots \ldots \ldots \ldots . . \ldots \ldots$

24. Final statistics for zinc in the composite Mining Waste Sequential Batch Extracts, $\mu g / g \ldots \ldots \ldots \ldots \ldots \ldots \ldots \ldots \ldots \ldots . . . \ldots$

25. Final statistics for Aluminum in the spray Dryer Waste

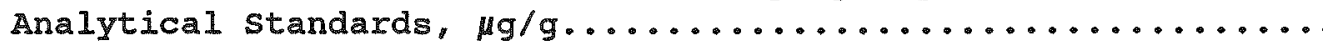

26. Final statistics for Barium in the spray Dryer Waste

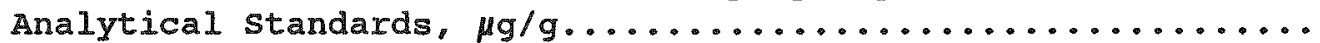

27. Final statistics for Boron in the spray Dryer Waste

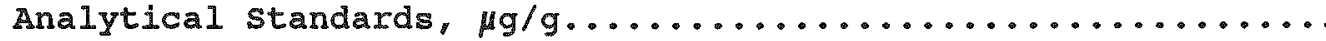

28. Final statistics for Calcium in the spray Dryer waste

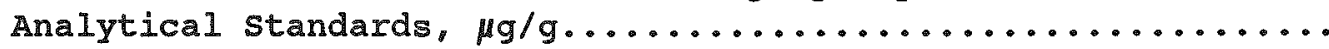

29. Final statistics for Chromium in the Spray Dryer Waste High Concentration Analytical standard, $\mu g / g \ldots . . \ldots \ldots \ldots . . . \ldots . .$.

30. Final statistics for silicon in the spray Dryer Waste

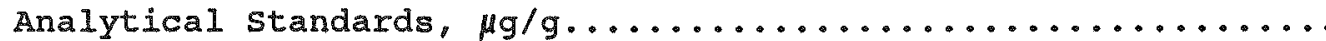

31. Final statistics for sodium in the spray Dryer waste

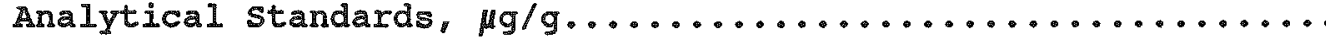

32. Final statistics for strontium in the spray Dryer Waste

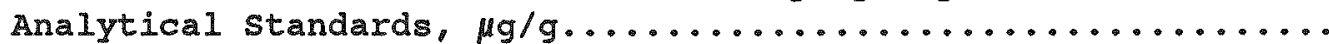

33. Final statistics for Barium in the Composite Mining Waste

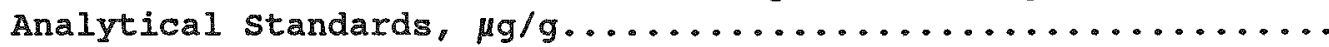

34. Final statistics for calcium in the composite Mining Waste

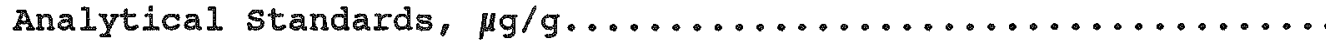




\section{LIST OF TABLES (continued)}

35. Final statistics for Lead in the composite Mining Waste

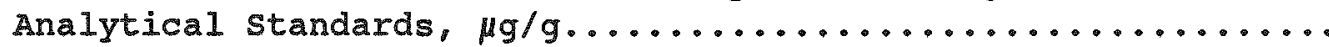

36. Final statistics for Magnesium in the composite Mining waste

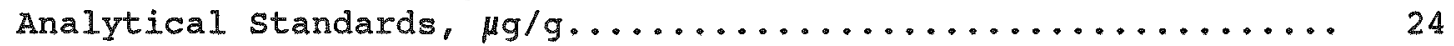

37. Final statistics for Manganese in the composite Mining waste

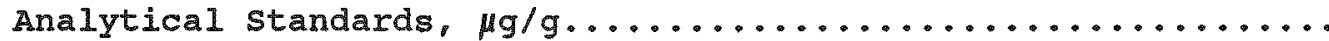

38. Final statistics for silicon in the Composite Mining Waste

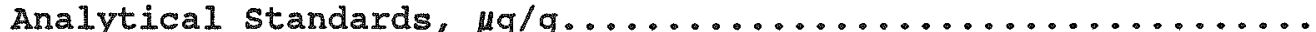

39. Final statistics for zinc in the composite Mining Waste Analytical standards, $\mu g / g \ldots \ldots \ldots \ldots \ldots \ldots \ldots \ldots \ldots \ldots \ldots . . \ldots \ldots \ldots$

40. Final statistics for Aluminum in the spray Dryer Waste special Analytical samples, $\mu g / g \ldots . . \ldots \ldots \ldots \ldots \ldots \ldots \ldots . . . \ldots$

41. Final statistics for Barium in the spray Dryer Waste special Analytical samples, $\mu g / g \ldots . . . . . . . . . . . . . . . .$.

42. Final statistics for Boron in the spray Dryer Waste special Analytical samples, $\mu g / g \ldots \ldots \ldots \ldots \ldots \ldots \ldots \ldots \ldots \ldots \ldots \ldots . . \ldots$

43. Final statistics for Calcium in the spray Dryer Waste special Analytical samples, $\mu g / g . \ldots . \ldots . \ldots \ldots \ldots . . . . . . .$.

44. Final statistics for chromium in the spray Dryer Waste

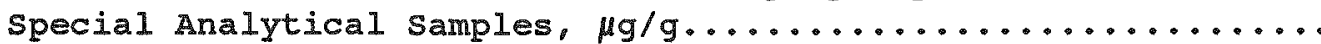

45. Final statistics for silicon in the spray Dryer Waste Special Analytical samples, $\mu g / g \ldots \ldots \ldots \ldots \ldots \ldots \ldots \ldots . . \ldots \ldots \ldots$

46. Final statistics for sodium in the spray Dryer Waste special Analytical samples, $\mu g / g \ldots \ldots \ldots \ldots \ldots \ldots \ldots \ldots \ldots \ldots . . . \ldots \ldots$

47. Final statistics for strontium in the spray Dryer waste

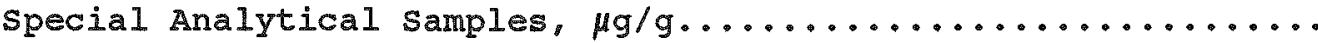

48. Final statistics for Barium in the Composite Mining Waste

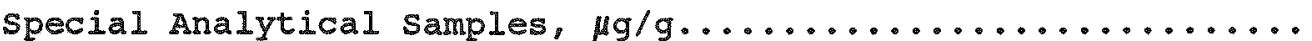

49. Final statistics for Calcium in the composite Mining waste

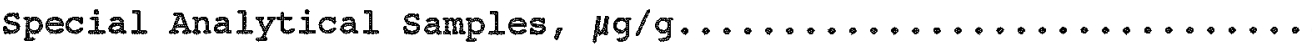

50. Final statistics for Lead in the composite Mining waste

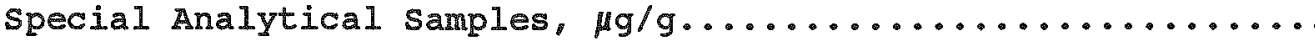

51. Final statistics for Magnesium in the composite Mining Waste

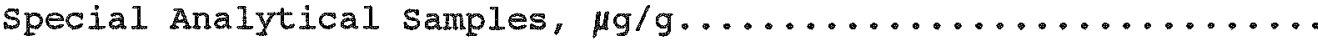




\section{LIST OF TABLES (continued)}

52. Final statistics for Manganese in the Composite Mining Waste special Analytical Samples, $\mu \mathrm{g} / \mathrm{g} . \ldots \ldots \ldots \ldots \ldots \ldots \ldots \ldots \ldots$

53. Final statistics for silicon in the composite Mining Waste Special Analytical samples, $\mu g / g \ldots \ldots \ldots \ldots \ldots \ldots \ldots \ldots \ldots \ldots \ldots$

54. Final statistics for zinc in the composite Mining waste

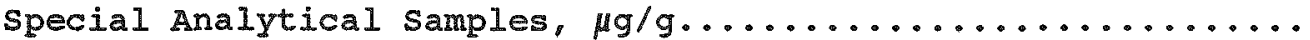

55. Estimated Multiple-Laboratory Precision of the sequential Batch Extraction Method Using Acidic Extraction Fluid in Extracting Aluminum from the Spray Dryer Waste, $\mu \mathrm{g} / \mathrm{g} . . . \ldots \ldots$

56. Estimated Multiple-Laboratory Precision of the sequential Batch Extraction Method Using Acidic Extraction Fluid in Extracting Barium from the spray Dryer Waste, $\mu g / g . . . \ldots \ldots \ldots$

57. Estimated Multiple-Laboratory Precision of the sequential Batch Extraction Method Using Acidic Extraction Fluid in Extracting Boron from the spray Dryer Waste, $\mu \mathrm{g} / \mathrm{g} \ldots . . \ldots \ldots \ldots$

58. Estimated Multiple-Laboratory Precision of the sequential Batch Extraction Method Using Acidic Extraction Fluid in Extracting Calcium from the spray Dryer Waste, $\mu \mathrm{g} / \mathrm{g} . . . \ldots \ldots \ldots$

59. Estimated Multiple-Laboratory Precision of the sequential Batch Extraction Method Using Acidic Extraction Fluid in Extracting Chromium from the spray Dryer waste, $\mu \mathrm{g} / \mathrm{g} . . . . . .$.

60. Estimated Multiple-Laboratory Precision of the Sequential Batch Extraction Method Using Acidic Extraction Fluid in Extracting silicon from the spray Dryer Waste, $\mu g / g . . . . . . .$.

61. Estimated Multiple-Laboratory Precision of the sequential Batch Extraction Method Using Acidic Extraction Fluid in Extracting sodium from the spray Dryer Waste, $\mu \mathrm{g} / \mathrm{g} \ldots . . \ldots \ldots \ldots$

62. Estimated Multiple-Laboratory Precision of the sequential Batch Extraction Method Using Acidic Extraction Fluid in Extracting Strontium from the spray Dryer Waste, $\mu \mathrm{g} / \mathrm{g} . . . . . .$.

63. Estimated Multiple-Laboratory Precision of the sequential Batch Extraction Method Using Acidic Extraction Fluid in Extracting Barium from the Composite Mining Waste, $\mu \mathrm{g} / \mathrm{g} . \ldots .$.

64. Estimated Multiple-Laboratory Precision of the sequential Batch Extraction Method Using Acidic Extraction Fluid in Extracting Calcium from the Composite Mining Waste, $\mu \mathrm{g} / \mathrm{g} . . . .$.

65. Estimated Multiple-Laboratory Precision of the Sequential Batch Extraction Method Using Acidic Extraction Fluid in Extracting Lead from the Composite Mining Waste, $\mu \mathrm{g} / \mathrm{g} . . . \ldots .$. 
66. Estimated Multiple-Laboratory Precision of the Sequential Batch Extraction Method Using Acidic Extraction Fluid in Extracting Magnesium from the Composite Mining

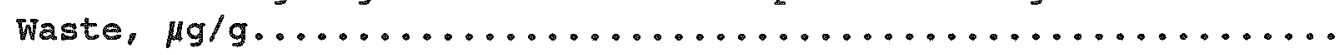

67. Estimated Multiple-Laboratory Precision of the sequential Batch Extraction Method Using Acidic Extraction Fluid in Extracting Manganese from the Composite Mining Waste, $\mu g / g . \ldots \ldots \ldots \ldots \ldots \ldots \ldots \ldots \ldots \ldots \ldots \ldots \ldots \ldots \ldots$

68. Estimated Multiple-Laboratory Precision of the sequential Batch Extraction Method Using Acidic Extraction Fluid in Extracting silicon from the Composite Mining Waste, $\mu \mathrm{g} / \mathrm{g} . . . .$.

69. Estimated Multiple-Laboratory Precision of the sequential Batch Extraction Method Using Acidic Extraction Fluid in Extracting zine from the Composite Mining Waste, $\mu \mathrm{g} / \mathrm{g} . . . . .$.

70. Estimated Single-operator Precision of the sequential Batch Extraction Method Using Acidic Extraction Fluid in Extracting Aluminum from the Spray Dryer

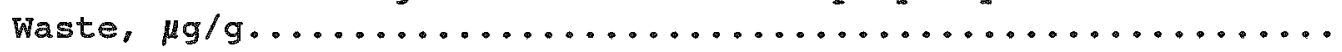

71. Estimated Single-operator Precision of the sequential Batch Extraction Method Using Acidic Extraction Fluid in Extracting Barium from the spray Dryer

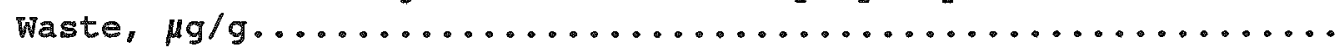

72. Estimated Single-operator Precision of the sequential Batch Extraction Method Using Acidic Extraction Fluid in Extracting Boron from the spray Dryer

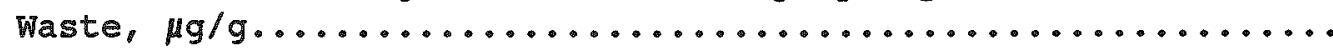

73. Estimated single-operator Precision of the sequential Batch Extraction Method Using Acidic Extraction Fluid in Extracting Calcium from the spray Dryer Waste, $\mu g / g . \ldots \ldots \ldots \ldots \ldots \ldots \ldots \ldots \ldots \ldots \ldots \ldots \ldots \ldots \ldots \ldots$

74. Estimated Single-operator precision of the sequential Batch Extraction Method Using Acidic Extraction Fluid in Extracting Chromium from the spray Dryer

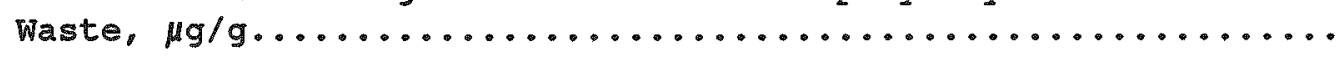

75. Estimated Single-operator Precision of the sequential Batch Extraction Method Using Acidic Extraction Fluid in Extracting silicon from the spray Dryer

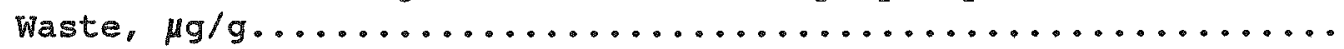

76. Estimated Single-Operator Precision of the sequential Batch Extraction Method Using Acidic Extraction Fluid in Extracting Sodium from the spray Dryer Waste, $\mu g / g \ldots \ldots \ldots \ldots \ldots \ldots \ldots \ldots \ldots \ldots \ldots \ldots \ldots \ldots . \ldots \ldots$ 


\section{LIST OF TABLES (continued)}

77. Estimated single-operator Precision of the Sequential Batch Extraction Method Using Acidic Extraction Fluid in Extracting strontium from the spray Dryer

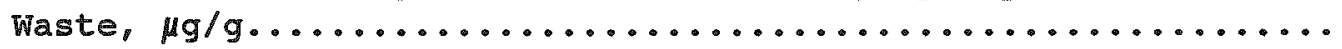

78. Estimated Single-operator Precision of the Sequential Batch Extraction Method Using Acidic Extraction Fluid in Extracting Barium from the Composite Mining Waste, $\mu g / g \ldots \ldots \ldots \ldots \ldots \ldots \ldots \ldots \ldots \ldots \ldots \ldots \ldots \ldots \ldots \ldots$

79. Estimated Single-operator Precision of the sequential Batch Extraction Method Using Acidic Extraction Fluid in Extracting Calcium from the Composite Mining Waste, $\mu g / g . \ldots \ldots \ldots \ldots \ldots \ldots \ldots \ldots \ldots \ldots \ldots \ldots \ldots \ldots \ldots . . \ldots \ldots$

80. Estimated Single-operator Precision of the sequential Batch Extraction Method Using Acidic Extraction Fluid in Extracting Lead from the Composite Mining Waste,$\mu g / g . \ldots \ldots \ldots \ldots \ldots \ldots \ldots \ldots \ldots \ldots \ldots \ldots \ldots \ldots \ldots$

81. Estimated single-operator Precision of the sequential Batch Extraction Method Using Acidic Extraction Fluid in Extracting Magnesium from the Composite Mining Waste, $\mu \mathrm{g} / \mathrm{g} . \ldots \ldots \ldots \ldots \ldots \ldots \ldots \ldots \ldots \ldots \ldots \ldots \ldots \ldots \ldots . . . \ldots \ldots$

82. Estimated Single-operator Precision of the seguential Batch Extraction Method Using Acidic Extraction Fluid in Extracting Manganese from the Composite Mining

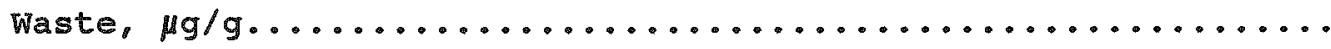

83. Estimated single-operator precision of the sequential Batch Extraction Method Using Acidic Extraction Fluid in Extracting Silicon from the composite Mining

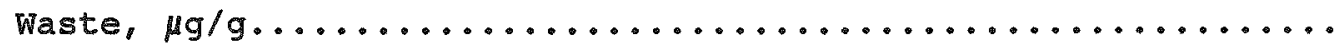

84. Estimated Single-operator Precision of the sequential Batch Extraction Method Using Acidic Extraction Fluid in Extracting zine from the composite Mining

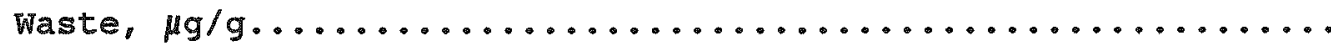

85. Statistical Evaluation of the Data from Analysis of the Spray Dryer Waste special Analytical samples for

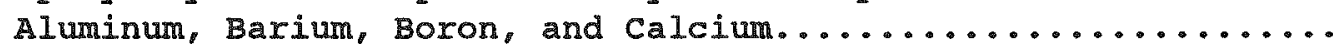

86. Statistical Evaluation of the Data from Analysis of the spray Dryer Waste special Analytical samples for Chromium, silicon, sodium, and strontium................

87. Statistical Evaluation of the Data from Analysis of the Composite Mining waste special Analytical Samples for

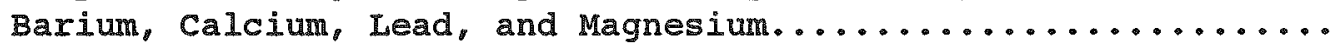




\section{IIST OF TABLES (continued)}

88. Statistical Evaluation of the Data from Analysis of the Composite Mining Waste special Analytical samples for

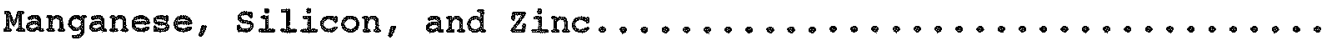

89. Evaluation of the Effects of stopping the Sequential Batch Extraction Method Over the Weekend Spray Dryer Waste: Extract $1 \ldots \ldots \ldots \ldots \ldots \ldots \ldots \ldots \ldots \ldots$

90. Evaluation of the Effects of stopping the Sequential Batch Extraction Method Over the Weekend Spray Dryer Waste: Extract $2 \ldots \ldots \ldots \ldots \ldots \ldots \ldots \ldots \ldots \ldots \ldots$

91. Evaluation of the Effects of stopping the sequential Batch Extraction Method over the weekend Spray Dryer Waste: Extract $3 \ldots \ldots \ldots \ldots \ldots \ldots \ldots \ldots \ldots \ldots \ldots \ldots$

92. Evaluation of the Effects of stopping the sequential Batch Extraction Method over the weekend Spray Dryer Waste: Extract $4 \ldots \ldots \ldots \ldots \ldots \ldots \ldots \ldots \ldots \ldots \ldots$

93. Evaluation of the Effects of stopping the Sequential Batch Extraction Method over the Weekend

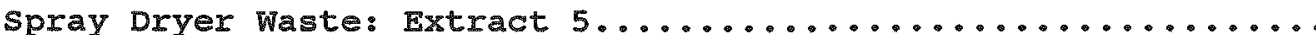

94. Evaluation of the Effects of stopping the sequential Batch Extraction Method Over the Weekend Composite Mining Waste: Extract $1 \ldots \ldots \ldots \ldots \ldots \ldots \ldots \ldots \ldots$

95. Evaluation of the Effects of stopping the sequential Batch Extraction Method Over the Weekend Composite Mining Waste: Extract $2 \ldots \ldots \ldots \ldots \ldots \ldots \ldots \ldots$

96. Evaluation of the Effects of stopping the sequential Batch Extraction Method over the Weekend Composite Mining Waste: Extract $3 \ldots \ldots \ldots \ldots \ldots \ldots \ldots \ldots \ldots$

97. Evaluation of the Effects of stopping the sequential

Batch Extraction Method over the Weekend

Composite Mining Waste: Extract $4 \ldots \ldots \ldots \ldots \ldots \ldots \ldots \ldots \ldots$

98. Evaluation of the Effects of stopping the sequential Batch Extraction Method over the Weekend Composite Mining Waste: Extract $5 \ldots \ldots \ldots \ldots \ldots \ldots \ldots \ldots \ldots \ldots$

99. Average pH Values of Control Extracts and Collaborative

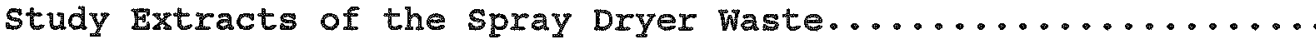

100. Average pH Values of Control Extracts and Collaborative study Extracts of the Composite Mining Waste................

101. Determination of Analytical Bias for Aluminum in the spray

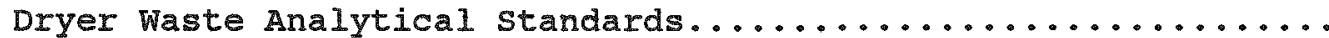


102. Determination of Analytical Bias for Barium in the spray Dryer Waste Analytical standards.....................

103. Determination of Analytical Bias for Boron in the spray

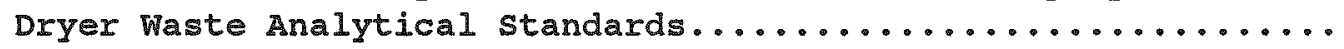

104. Determination of Analytical Bias for Calcium in the spray

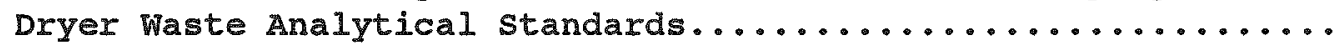

105. Determination of Analytical Bias for Chromium in the spray

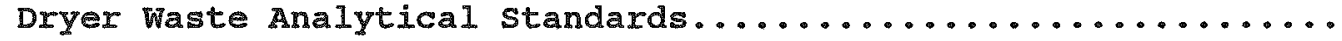

106. Determination of Analytical Bias for silicon in the spray

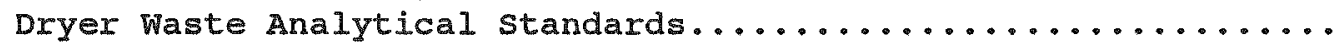

107. Determination of Analytical Bias for sodium in the spray

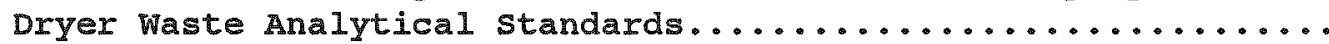

108. Determination of Analytical Bias for strontium in the spray

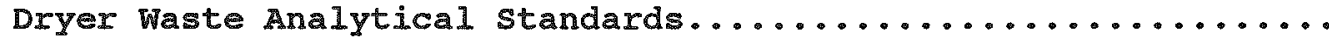

109. Determination of Analytical Bias for Barium in the Composite

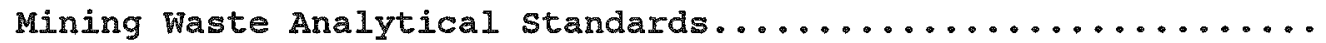

110. Determination of Analytical Bias for Calcium in the Composite Mining Waste Analytical standards...............

111. Determination of Analytical Bias for Lead in the Composite

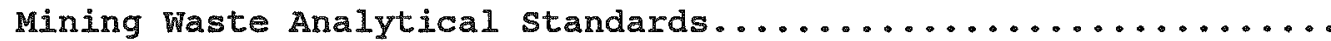

112. Determination of Analytical Bias for Magnesium in the

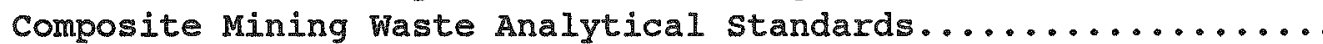

113. Determination of Analytical Bias for Manganese in the Composite Mining Waste Analytical standards...............

114. Determination of Analytical Bias for silicon in the Composite Mining Waste Analytical standards................

115. Determination of Analytical Bias for zinc in the Composite

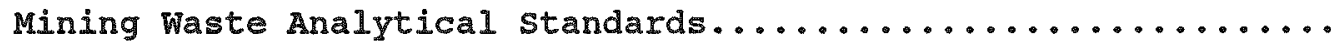


Appendix A. Test Method Used by the Participants in the

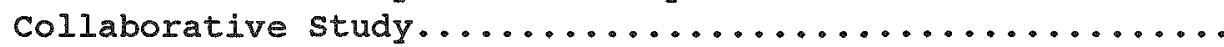

Appendix B. List of Participating Laboratories in the

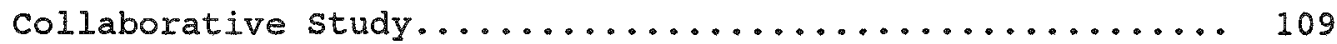

Appendix c. Collaborative study Instructions.................. 111

Appendix D. Collaborative study Questionnaire and sumary of Responses......................... 114

Appendix E. Collaborative study Data Summary sheets............... 119

Appendix F. ASTM Method D5284-92, standard Test Method for Sequential Batch Extraction of Waste with Acidic

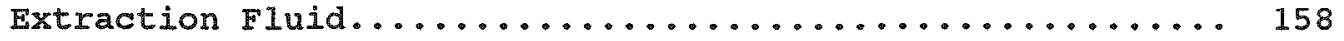

Appendix G. Precision and Bias statements to be Added to ASTM

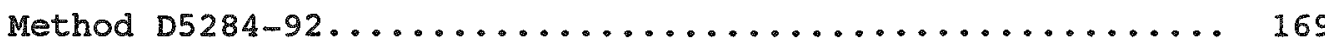


1. Estimated Precision of the sequential Batch Extraction Method for Aluminum in the spray Dryer Waste.............. 48

2. Estimated Precision of the sequential Batch Extraction Method for Barium in the spray Dryer waste................4 48

3. Estimated Precision of the sequential Batch Extraction Method for Boron in the spray Dryer waste.................550

4. Estimated Precision of the Sequential Batch Extraction Method for calcium in the spray Dryer waste............... 50

5. Estimated Precision of the sequential Batch Extraction Method for chromium in the spray Dryer waste...............

6. Estimated Precision of the sequential Batch Extraction Method for silicon in the spray Dryer waste................

7. Estimated Precision of the sequential Batch Extraction

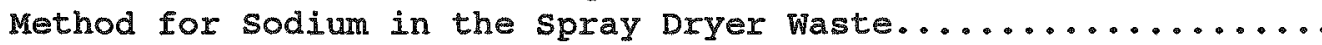

8. Estimated Precision of the sequential Batch Extraction Method for strontium in the spray Dryer waste..............

9. Estimated Precision of the sequential Batch Extraction Method for Barium in the composite Mining waste............

10. Estimated Precision of the sequential Batch Extraction Method for calcium in the composite Mining waste............ 55

11. Estimated Precision of the sequential Batch Extraction Method for Lead in the Composite Mining waste..............

12. Estimated Precision of the seguential Batch Extraction Method for Magnesium in the composite Mining Waste...........

13. Estimated Precision of the sequential Batch Extraction Method for Manganese in the Composite Mining Waste..........

14. Estimated Precision of the sequential Batch Extraction Method for silicon in the composite Mining Waste............

15. Estimated Precision of the Sequential Batch Extraction Method for zinc in the Composite Mining waste............... 
This project was conducted to develop and validate a sequential batch extraction method using a dilute acid solution as the extraction fluid. The project objectives were to (1) develop a sequential batch extraction procedure using extraction fluids simulating the composition and $\mathrm{pH}$ of acid rain, (2) determine the precision of the extraction procedure when applied to specific waste materials by conducting a collaborative study using the method, and (3) ballot the method within the American society for Testing and Materials (ASTM) to provide a new ASTM standard test method.

A draft method for sequential batch extraction of waste with an acidic extraction fluid was written by ASTM Task Group D34.02.01 on Waste Leaching Techniques. This method calls for mixing a known mass of waste with an acidic extraction fluid, prepared using a $60 / 40$ wt 8 mixture of sulfuric acid/nitric acid solution, and separating the liquid phase for analysis or testing. The $\mathrm{pH}$ of the extraction fluid is to reflect the $\mathrm{pH}$ of acidic precipitation in the geographic region where the material being tested is to be disposed. The procedure is conducted ten times in sequence on the same sample of material and generates ten extracts. These extracts can be analyzed for specific constituents or properties, used for biological testing procedures, etc. Use of the extracts is to be determined by the user of the method. The method can be applied to any material containing at least 5\% dry solids.

The draft method was balloted within ASTM Subcomnittee D34.02 on Physical and Chemical characterization and ASTM Main Committee D 44 on Waste Management. After passing these balloting processes, the method was balloted within the ASTM Society and passed society balloting on August 15, 1992. This new ASTM test method is designated as ASTM Method D5284-92, standard Test Method for Sequential Batch Extraction of Waste with Acidic Extraction Fluid.

A collaborative study, in which the method was used to extract two different waste materials, a spray dryer waste from an innovative clean coal technology process and a composite mining waste, using two different extraction fluid pH values, was conducted to determine the multiplelaboratory and single-operator precision of the extraction procedure. In the collaborative study, data were also generated for evaluating the effects of filter pore size and digestion versus nondigestion on analytical concentrations in the extracts from the test materials.

Based on the data generated by the collaborative study, precision and bias statements and information obtained from the study were prepared for addition to the new method. These were balloted concurrently within ASTM subcomittee D34.02 on Physical and chemical Characterization and ASTM Main Committee D34 on Waste Management as a modification to ASTM Method D5284-92. The proposed addition to the new method passed subcommittee and main committee balloting and will be balloted within the ASTM society in August 1993. Society approval is expected, and the precision and bias statements should be published as part of ASTM Method D5284-92 in 1994 . 
A general precision statement that covers all wastes, analytical parameters, and extraction fluid pH values can not be prepared because the data generated in the collaborative study are specific to the test materials, extraction fluid $\mathrm{pH}$ values, and elements of interest in this study. However, the data generated in this study give the user information on both multiplelaboratory and single-operator precision of the extraction procedure when it is applied to two different waste materials using two different extraction fluid pH values. As a result, the precision statement for this method provides information on the variability that can be expected between test results when the method is used by one or more laboratories. This information will help potential users assess the usefulness of the method in their proposed applications. The data also provide information to aid the user in making decisions concerning filtering the extraction slurries and analyzing the extracts.

Based on the information obtained in this study, the following conclusions can be drawn.

- The estimated precision of the sequential batch extraction method using acidic extraction fluid varies with the type of waste being tested and the element of interest.

- For the materials tested in this study, the estimated multiplelaboratory precision of the sequential batch extraction method for extracting many of the elements of interest varies somewhat with the elemental concentration extracted from the test material, and in many of these cases, there is a direct relationship between estimated multiple-laboratory standard deviation and extract concentration.

- For most elenents of interest in this study, the estimated precision of the extraction procedure varies with the extraction number in the sequence.

- For the test materials and elements of interest in this study, the estimated single-operator precision of the extraction method in many cases is much better than the estimated multiple-laboratory precision.

- Filter pore size, $0.45-\mu \mathrm{m}$ versus $0.8-\mu \mathrm{m}$, and digestion versus nondigestion affect certain elemental concentrations determined in the extracts of the spray dryer waste and composite mining waste. The effects of these variables on the elemental concentrations are waste and element specific.

- Elemental solubility in sequential batch water extracts versus acid solution extracts depends on the solution chemistry of the extraction slurries. As a result, the effect of using an acidic extraction fluid versus water depends on the material being tested, the $\mathrm{pH}$ of the extraction fluid, the element or parameter(s) of interest, and the extraction number in the sequence of the sequential batch extraction. 


\section{INTRODUCTION}

Single batch extraction methods, such as the Extraction Procedure (EP) Toxicity Test (U.S. EPA 1980), ASTM Method D3987 (ASTM 1992), and the Toxicity Characteristic Leaching Procedure (TCLP) (U.S. EPA 1990), are commonly used to characterize waste materials. A single batch extraction procedure can give useful information concerning the constituents that can be extracted from a material during its initial contact with a leaching medium. However, to evaluate the leaching characteristics of material during repeated exposure to an extraction fluid, a method involving multiple leaching sequences is useful. For example, sequential batch extractions can indicate whether or not the majority of soluble constituents is leached in the first extraction step, or if the buffering capacity of a matrix decreases with repeated exposure to the leaching fluid. For these reasons, a sequential batch extraction procedure, ASTM Method D4793 (ASTM 1992), was developed and validated as an ASTM standard method.

This method contains great detail and explanation to prevent confusion in performing the extraction steps and to ensure that with each sequence, the correct solid-to-liquid ratio is maintained. However, ASTM Method D4793 uses water as the extraction fluid, and the increasing occurrence of acid rain throughout the world has resulted in a need for a detailed, multiple-step extraction procedure involving extraction fluids simulating acid rain solutions.

The purpose of this project was to develop a sequential batch extraction method using a dilute acid solution as the extraction fluid; and to conduct a collaborative study using the method to determine its precision when applied to two different waste materials using two different extraction fluid $\mathrm{pH}$ values. Data generated in the collaborative study are to aid the user of the method by providing information on the variability that can be expected when the method is used by one or more laboratories.

The sequential batch extraction procedure that was developed and validated requires an extraction fluid having a pH representative of precipitation in the geographic region where the material being tested is to be placed. The new standard allows flexibility in selection of extraction fluid $\mathrm{pH}$ in an attempt to more closely represent site-specific conditions. The method can be used to evaluate the buffering capacity of materials repeatedly in contact with acidic media, such as acid rain, and provides a relatively fast and inexpensive procedure for examining the effects of acidic media on the leachability of various waste types and treated waste materials. It can be used as a screening method or in conjunction with long-term modeling or field studies. Sequential batch extraction data are useful for site assessment, for modeling source term depletion, and for risk assessment.

\section{DESCRIPTION OF THE WORK}

\section{Project objectives}

The purpose of this research was to develop and validate a sequential batch extraction method using dilute acid solution as the extraction fluid. The project objectives were to (1) develop a sequential batch extraction 
procedure using extraction fluids simulating the composition and pH of acid rain, (2) determine the precision of the extraction procedure when applied to specific waste materials by conducting a collaborative study using the method, and (3) ballot the method within ASTM to provide a new ASTM standard test method.

\section{Summary of Literature Review}

Single batch extraction methods are commonly used to characterize the leachability of materials. These methods include the Extraction Procedure (EP) Toxicity Test (U.S. EPA 1980), ASTM Method D3987, standard Test Method for shake Extraction of solid waste with water (ASTM 1992), the Toxicity Characteristic Leaching Procedure (TCLP) (U.S. EPA 1990), the California Waste Extraction Test (WET) (California Environmental Protection Agency 1991), and the Canadian Leachate Extraction Procedure (Canadian General standard Board 1987). These procedures vary in their test parameters and extraction fluids.

ASTM Method D3987 uses reagent water as the extraction fluid to simulate leaching due to contact with groundwater and precipitation. Depending on the "alkalinity" of the sample, the TCLP uses either a sodium acetate buffer solution having a $\mathrm{pH}$ of $4.93 \pm 0.05$ or a dilute acetic acid solution having a $\mathrm{pH}$ of $2.88 \pm 0.05$ as the extraction fluid. These extraction fluids are to simulate leaching conditions in a disposal site containing 958 municipal waste and $5 \%$ industrial waste. The California WET uses a $0.2 \mathrm{M}$ sodium citrate solution having a pH of $5.0+0.1$ as the extraction fluid. The citric acid buffer is used because it is a common metal complexing agent found naturally in soil and water. When using these methods, the final pH of the extract reflects the interaction of the extractant with the buffering capacity of the waste. The EP Toxicity Test and the Canadian Leachate Extraction Procedure both use addition of $0.5 \mathrm{~N}$ acetic acid solution (up to a volume of four times the sample mass) to maintain the $\mathrm{pH}$ of the extraction slurry at $5.0 \pm 0.2$. Acetic acid is a common organic acid found in landfills.

All of these methods provide information on the constituents that can be extracted from a material during its initial contact with a specific leaching medium. However, they do not provide information on the leaching characteristics of the material during repeated exposure to the extraction fluid. In addition, these methods do not provide extraction fluid flexibility to address site-specific disposal conditions.

There is a single batch extraction method in which the extraction fluid used is a function of the region of the united states where the sample site is located. This is the synthetic precipitation Leaching Procedure (U.S. EPA Method 1312) (U.S. EPA 1990). An extraction fluid, prepared by adding a $60 / 40$ wt mixture of sulfuric and nitric acids to reagent water to give a pH of $4.20 \pm 0.05$, is used to test samples from sites located east of the Mississippi River, and an extraction fluid, prepared in the same manner but having a pH of $5.00 \pm 0.05$, is used to test samples from sites located west of the Mississippi River. As with the other single batch extraction procedures, this method does not provide information on whether or not the majority of soluble constituents is leached in the first extraction step, or if the buffering capacity of the matrix decreases with repeated exposure to the extraction fluid. 
U.S. Environmental Protection Agency (EPA) Method 1320, Multiple Extraction Procedure (U.S. EPA 1990), is intended to simulate the leaching a waste will undergo from repeated contact with acid precipitation when disposed in an improperly designed sanitary landfill. This EPA method specifies use of a single extraction fluid having a pH of $3.0 \pm 0.2$. This gives no flexibility in extraction fluid pH, and as a result, regional variability in the $\mathrm{pH}$ of precipitation (Environment Canada Federal/Provincial Research and Monitoring Coordination Committee 1990) is ignored. In addition, Method 1320 provides very little detail on how to perform the multiple extractions and contains no data on method performance.

RecentIy, ASTM Method D4793, Standard Test Method for sequential Batch Extraction of Waste with water (ASTM 1992), was developed and validated. This method provides the detail and explanation necessary to prevent confusion in performing the sequential batch extraction procedure and to ensure that the correct solid-to-liquid ratio is maintained from sequence-tosequence. However, this procedure uses water as the extraction fluid. Increasing deposition of acid-forming constituents, sulfate and nitrate, from the atmosphere (Hidy 1987) generates a need for a sequential batch extraction method, which uses an extraction fluid containing sulfuric and nitric acids and having a pH representative of precipitation in the geographic region where the sample site is located.

\section{Project Plan}

The following tasks were performed to meet the project objectives. These tasks are described in detail in subsequent sections of this report.

Task 1. Develop a draft method for sequential batch extraction of waste with acidic extraction fluid.

Task 2. Ballot the draft method within ASTM subcommittee D34.02 on Physical and Chemical Characterization and address negative votes and comments from the balloting.

Task 3. Coordinate an interlaboratory experimental program by designing and conducting a collaborative study that will provide information on the precision of the method, as well as, other information that will be beneficial to users of the method.

Task 4. Ballot the draft method within ASTM Main Committee D34 on waste Management and address negative votes and comments from the balloting.

Task 5. Ballot the draft method at the society level of ASTM.

Development of the Sequential Batch Extraction Method Using Dilute Acid Solution

A draft method for sequential batch extraction of waste with acidic extraction fluid was written by ASTM Task Group D34.02.01 on waste Leaching Techniques. This method was developed by modifying ASTM Method D4793-88, standard Test Method for sequential Batch Extraction of Waste with Water, to use an acidic extraction fluid. The method calls for mixing a known mass of 
waste with an acidic extraction fluid, prepared using a 60/40 wt 8 mixture of sulfuric acid/nitric acid solution, and separating the liquid phase for analysis or testing. The acid ratio of $60 / 40$ wt 8 sulfuric acid/nitric acid was selected because about two-thirds of the acid in rainfall is sulfuric acid and one-third is nitric acid (Gordon 1987). The pH of the extraction fluid is to reflect the $\mathrm{pH}$ of acidic precipitation in the geographic region where the material being tested is to be placed. The procedure is conducted ten times in sequence on the same sample of material and generates ten solutions. These extracts can be analyzed for specific constituents or properties, used for biological testing procedures, etc. Use of the extracts is to be determined by the user of the method. The procedure can be applied to any material containing at least $5 \%$ dry solids.

After the task group prepared and reviewed the draft method, it was balloted within ASTM Subcommittee D34.02 on Physical and chemical characterization for subcommittee review. The task group incorporated comments and suggestions received from the subcommittee, and based on the favorable balloting results, recommended that the collaborative study to validate the method begin. A copy of the test method which was used by the participants in the collaborative study is included as Appendix $\mathbb{A}$. This version of the draft test method was then balloted once again at the subcommittee level.

As a result of the second subcommittee balloting, changes to the method concerning sample holding time from field collection to the first extraction step and the amount of sample that should be sent to the laboratory to ensure a representative sample for extraction were made (section 7). These changes required the method to be balloted once again at subcommittee level. However, since the changes dealt with sampling, they were not applicable to the collaborative study, and as a result, did not affect the study.

One other change to the draft method that was decided by the task group was to use a 50-gram sample of waste, rather than a 100-gram sample, for determination of solid content (section 9.2.2). Due to the nature of the test materials used in the collaborative study (dry powders), the solid contents determined for the two materials do not vary with the use of a $50-$ gram sample or a 100-gram sample. This is shown by the data listed in Table 1. These data were generated by performing duplicate solid content determinations, as specified in the draft method, using 50- and 100-gram samples of each test material.

Because technical changes were made to the method, it was necessary to once again ballot the method at the subcommittee level. It was recommended by the task group that the method be balloted concurrently within AstM subcommittee D34.02 and ASTM Main Committee D34 on Waste Management.

The voting results from the concurrent balloting of the draft method were discussed by the task group. Based on the comments received, technical changes were made to five sections of the method, and one note was changed to a section. Although additional technical changes were made to the draft method, these did not affect the application of the method in the collaborative study. 
Table 1. Percent Solid in the Spray Dryer Waste and the composite Mining Waste

\section{Spray Dryer waste}

Mass of Sample Used

$$
50.0 \mathrm{~g}
$$

$50.0 \mathrm{~g}$

100.09

100.09
Pexcent solid

$97.8 \%$

$98.2 \%$

98.08

97.8 8

Composite Mining Waste

Mass of Sample Used

$50.0 \mathrm{~g}$

$50.0 \mathrm{~g}$

$100.0 \mathrm{~g}$

100.09
Percent solid

99.68

99.28

99.68

99.48

One of the changes made to the method requires that the first four extraction sequences be performed without interruption. This was specified in the collaborative study. The second change made to the method specifies using 95-98 wt sulfuric acid and 69-71 wt 8 nitric acid to prepare the extraction fluid. This was added to reagent section 6.3 to clarify the term "concentrated" which is used in describing the reagents in section 9.3. The third change involved modifying the requirement of discarding extraction fluid that does not meet the pH specification to requiring that the extraction fluid not be used in the extraction procedure if it does not meet the pH specification. Another change involved changing the allowable temperature fluctuation specified for the drying oven in the apparatus section to a value that is consistent with the requirements given in the procedure. A \pm value for allowable temperature fluctuation was added to one of the sections requiring use of the oven. It was also brought up by the subcomittee that a note is not technically part of a method. As a result, the task group decided to change one of the notes in the method to a section.

Because only six sections of this lengthy method were technically changed, it was recommended by the subcomittee that only those sections be balloted, first within the subcommittee and then at main committee 1 evel. The results of the subcomittee balloting were 63 affirmative votes, zero negative votes, and 57 abstentions, and the results of the main committee balloting were 131 affirmative votes, zero negative votes, and 200 abstentions. The draft method was then balloted at society level and passed society balloting on August 15, 1992. The method is now designated as AsTM standard Test Method D5284-92. A copy of Method D5284-92 is included as Appendix F. 


\section{Collaborative study Design}

The collaborative study of this sequential batch extraction method was designed so an estimated analytical error could be determined and subtracted from the total error due to both performing the extraction method and analyzing the resulting extracts. In this way, the error due to the extraction procedure can be estimated. In the collaborative study, data were also generated for evaluating the effects of filter pore size and digestion versus nondigestion on analytical concentrations in the extracts from the test materials used in the study.

Ten laboratories participated individually or as teams in the collaborative study. The laboratories extracted two different waste materials in duplicate using extraction fluids having different pH values. The resulting extracts were then analyzed for specific inorganic analytes. six analytical standards were analyzed in triplicate to generate data for calculating analytical error. In addition, eight special samples were analyzed by the laboratories to generate data for evaluating the effects of filter pore size and digestion versus nondigestion on analytical values. Filter pore size can affect elemental concentrations in the extracts, and analytical values may be affected by sample digestion.

The laboratories that participated in the collaborative study are Battelle Pacific Northwest Laboratories, Browning-Ferris Industries, Chemical Waste Management, CORE Laboratories, Dell Engineering, Matrix Analytical, Resource Materials Testing, Union Carbide Corporation, U.S. Bureau of Mines spokane Research center, and western Research Institute. Additional information concerning the collaborative study participants is given in Appendix B.

In some cases, the laboratories teamed together to generate the collaborative study data. Resource Materials Testing teamed with CORE Laboratories by performing the extractions and sending the extracts to CORE Laboratories for analysis. The same was true for Union Carbide corporation and western Research Institute. Union Carbide generated extracts and Western Research Institute analyzed them. Browning-Ferris Industries and Western Research Institute also teamed together in the collaborative study. Browning-Ferris Industries performed the analytical portion of the study and extractions of one of the test materials, and Western Research Institute performed extractions of the other test material. In the collaborative study, the participants and teams of participants were randomly designated as laboratory \#1, \#2, \#3, etc. Eight complete data sets were generated by the laboratories in the collaborative study.

The collaborative study participants were sent a copy of the method to be performed (Appendix A), a list of special instructions to be followed in the study (Appendix C), laboratory report forms for recording their data, and a questionnaire on general information concerning performance of the method (Appendix D). In addition, the participants were sent a container of each of the two test materials, analytical standards for analysis, and special analytical samples for analysis. 


\section{Collaborative study Test Materials}

The two test materials used in the collaborative study were a spray dryer waste from an innovative clean coal technology process, designated as waste $\mathrm{U}$, and a composite mining waste, designated as waste $M$. These materials were evaluated for their usefulness in the study by extracting them using the draft method and reviewing the elemental concentrations in their extracts. The spray dryer waste was extracted using an extraction fluid having a pH of $4.3 \pm 0.05$, representing a pH value of acidic precipitation in the eastern United States (Environment Canada Federal/Provincial Research and Monitoring Coordination committee 1990). The composite mining waste was extracted using an extraction fluid having a pH of $5.0 \pm 0.05$, representing a pH value of acidic precipitation in the western united states (Environment canada Federal/Provincial Research and Monitoring Coordination Committee 1990).

The criteria used in selecting appropriate test materials for the study were that the materials should produce extracts having seven to ten elements with concentrations above instrumental detection limits in all ten extracts, and that these elements should include some of the metals regulated under the Resource Conservation and Recovery Act (RCRA) (1984). Nondigested extracts of potential test materials were analyzed using inductively coupled plasma (ICP) spectrometry (EPA Method 6010) (U.S. EPA 1990).

The spray dryer waste met the selection criteria based on the concentrations of eight elements in the ten extracts generated by extracting the waste with $\mathrm{pH} 4.3$ extraction fluid. These eight elements and their concentration ranges in the extracts are listed in Table 2 . The composite mining waste was selected for use in the collaborative study based on the concentrations of seven elements in the ten extracts generated by extracting this material with extraction fluid having a pH of 5.0. These seven elements and their concentration ranges in the extracts are listed in Table 3.

Table 2. Elemental Concentration Ranges in the spray Dryer Waste Extracts, ing/L

Element

Aluminum

Barium

Boron

Calcium

chromium

silicon

sodium

strontium
Concentration Range

$\begin{array}{llc}1.3 & - & 8.7 \\ 0.24 & - & 2.1 \\ 0.1 & - & 0.36 \\ 89 & - & 190 \\ 0.01 & - & 0.14 \\ 3.4 & - & 8.2 \\ 1.5 & - & 180 \\ 1.2 & - & 9.8\end{array}$


Table 3. Elemental concentration Ranges in the Composite Mining Waste Extracts, mg/L

Element

Barium

Calcium

Lead

Magnesium

Manganese

silicon

zinc
Concentration Range

$\begin{array}{ccc}0.03 & - & 0.96 \\ 11 & - & 83 \\ 0.15 & - & 4.5 \\ 0.21 & - & 5.0 \\ 0.02 & - & 2.0 \\ 0.41 & - & 2.0 \\ 0.01 & - & 17\end{array}$

Prior to preparing subsamples of the test materials to be sent to the participating laboratories in the collaborative study, a bulk quantity of each material was thoroughly mixed and tested for homogeneity. The spray dryer waste was mixed by rotating the material end-over-end at 30 rotations per minute for 14 days. After 14 days of mixing, 25 five-gram subsamples were withdrawn at random from the bulk material. Then the bulk sample was rotated end-over-end for an additional seven days. After seven days of mixing, one five-gram sample was withdrawn from the bulk material. The 26 samples of spray dryer waste were digested (EPA Method 3050) (U.S. EPA 1990) and analyzed for calcium using ICP spectrometry (EPA Method 6010) (U.S. EPA 1990). The data generated from analysis of the first 25 samples were used to establish a 958 confidence interval for the concentration of calcium in the spray dryer waste (Guttman et al. 1971). The concentration of calcium in the 26 th sample was compared to this $95 \%$ confidence interval. The criterion for homogeneity was if the concentration of calcium in the $26 \mathrm{th}$ sample fell within the $95 \%$ confidence interval, then the bulk material was homogeneous. Calcium was selected as the analyte for this determination because of its abundance in the spray dryer waste. Metal concentrations in the spray dryer waste are listed in Table 4. Results of the statistical evaluation of the data from the spray dryer waste homogeneity testing are listed in Table 5. These data suggest that the bulk sample of spray dryer waste had acceptable homogeneity for use in the collaborative study.

The same procedure was followed for the composite mining waste, except the bulk material was rotated end-over-end for three weeks before 25 subsamples were withdrawn. A longer initial mixing time was used because the mining waste was a composite sample containing various mining-related materials and may have required a longer mixing time to achieve the desired homogeneity. Calcium was also selected as the analyte of interest for this determination. Metal concentrations in the mining sample are listed in Table 6. The results of the statistical evaluation of the data generated in testing homogeneity of the composite mining waste are listed in Table 7. These data suggest that the bulk material had acceptable homogeneity for use in the collaborative study. 
Table 4. Metal Concentrations in the Spray Dryer waste

\begin{tabular}{lc}
\hline Metal & Concentration \\
\hline Aluminum & $8.05 \%$ \\
Barium & $0.376 \%$ \\
Boron & $659 \mathrm{mg} / \mathrm{kg}$ \\
Calcium & $23.5 \mathrm{~g}$ \\
Chromium & $45.3 \mathrm{mg} / \mathrm{kg}$ \\
Copper & $139 \mathrm{mg} / \mathrm{kg}$ \\
Iron & $3.48 \mathrm{8}$ \\
Lithium & $54.1 \mathrm{mg} / \mathrm{kg}$ \\
Magnesium & 3.098 \\
Nickel & $36.1 \mathrm{mg} / \mathrm{kg}$ \\
Silicon & $13.8 \mathrm{~g}$ \\
Sodium & $1.17 \mathrm{8}$ \\
Strontium & $0.248 \mathrm{~g}$ \\
Vanadium & $147 \mathrm{mg} / \mathrm{kg}$ \\
zinc & $123 \mathrm{mg} / \mathrm{kg}$ \\
\hline
\end{tabular}

Table 5. Spray Dryer waste Homogeneity

25 samples analyzed for calcium

$$
\begin{aligned}
& \overline{\mathrm{x}}=20.28 \\
& \mathrm{~s}=0.59 \\
& 95 \% \text { confidence interval }=20.0 \%-20.4 \%
\end{aligned}
$$

After additional mixing, 1 sample withdrawn and analyzed, calcium concentration $=20.18$

Table 6. Metal Concentrations in the Composite Mining Waste

\begin{tabular}{ll}
\hline Metal & Concentration \\
\hline Arsenic & $0.23 \mathrm{mg} / \mathrm{g}$ \\
Aluminum & $0.78 \mathrm{mg} / \mathrm{g}$ \\
Boron & $0.04 \mathrm{mg} / \mathrm{g}$ \\
Barium & $0.34 \mathrm{mg} / \mathrm{g}$ \\
Calcium & $1.03 \mathrm{~g}$ \\
Cadmium & $0.02 \mathrm{mg} / \mathrm{g}$ \\
Chromium & $0.03 \mathrm{mg} / \mathrm{g}$ \\
Copper & $0.48 \mathrm{mg} / \mathrm{g}$ \\
Iron & $1.99 \mathrm{~s}$ \\
Magnesium & $2.29 \mathrm{mg} / \mathrm{g}$ \\
Manganese & $1.88 \mathrm{mg} / \mathrm{g}$ \\
Lead & $3.77 \mathrm{~s}$ \\
Silicon & $0.51 \mathrm{mg} / \mathrm{g}$ \\
Zinc & $3.27 \mathrm{mg} / \mathrm{g}$ \\
\hline
\end{tabular}


Table 7. Composite Mining Waste Homogeneity

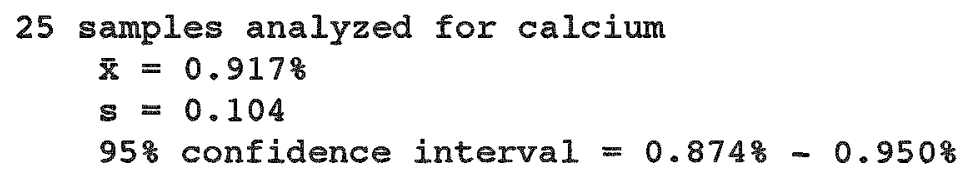

After additional mixing, 1 sample withdrawn and analyzed, calcium concentration $=0.9248$

\section{Collaborative study Analytical standards}

Based on the elemental concentrations in the sequential batch extracts of the spray dryer waste and the composite mining waste, low, medium, and high concentration analytical standards containing the elemental concentrationslisted in Tables 8 and 9 were prepared. The standards listed in Table 8 had a dilute acid solution matrix of $\mathrm{pH} 4.3$, and the standards listed in Table 9 had a dilute acid solution matrix of $\mathrm{pH} 5.0$. The acidic matrices of the standards were prepared as specified in the draft method (Appendix A). The standards were sent to the participating laboratories to be analyzed in triplicate using the same analytical method used to analyze the extracts.

Table 8. Elemental Concentrations in the spray Dryer waste Analytical standards, mg/L

\begin{tabular}{lccc}
\hline Element & Low standards & Medium Standards & High standards \\
\hline Aluminum & 1.5 & 6.0 & 8.5 \\
Barium & 0.25 & 1.5 & 2.5 \\
Boron & 0.15 & 0.2 & 0.4 \\
Calcium & 100 & 130 & 200 \\
Chromium & 0.01 & 0.03 & 0.15 \\
Silicon & 3.5 & 5.0 & 8.0 \\
Sodium & 1.5 & 5.0 & 180 \\
strontium & 1.0 & 2.5 & 10.0 \\
\end{tabular}

Table 9. Elemental Concentrations in the Composite Mining Waste Analytical standards, $\mathrm{mg} / \mathrm{L}$

\begin{tabular}{lccc}
\hline Element & Low standards & Medium Standards & High Standards \\
\hline Barium & 0.05 & 0.25 & 0.90 \\
Calcium & 12.0 & 30.0 & 80.0 \\
Lead & 0.25 & 1.5 & 4.5 \\
Magnesium & 0.30 & 0.65 & 5.0 \\
Manganese & 0.05 & 0.30 & 2.0 \\
Silicon & 0.50 & 1.5 & 2.0 \\
zinc & 0.05 & 2.0 & 15.0 \\
& & & \\
\hline
\end{tabular}




\section{Collaborative study Special Analytical samples}

special analytical samples were prepared for analysis by the participating laboratories. Four extractions of the spray dryer waste and four extractions of the composite mining waste were performed using the first extraction step in the draft method (Appendix $A$ ). Two extracts from each waste were generated by filtering the extraction slurries through $0.45-\mu \mathrm{m}$ pore-size filters, and two extracts from each waste were generated by filtering the extraction slurries through $0.8-\mu \mathrm{m}$ pore-size filters. These are common filter pore sizes used in separating the liquid extraction phase for analysis. One of the $0.45-\mu \mathrm{m}$ extracts and one of the $0.8-\mu \mathrm{m}$ extracts from each waste were digested (EPA Method 3010) (U.S. EPA 1990). The 0.8- $\mu \mathrm{m}$ and $0.45-\mu \mathrm{m}$ filtrates and digestates were divided into eight portions and sent to the collaborative study participants for analysis to generate data for evaluating the effects of filter pore size and digestion versus nondigestion on elemental concentrations in the extracts.

\section{Collaborative study Control samples}

To generate baseline data to be compared with the data generated in the collaborative study, the composite mining waste and the spray dryer waste were extracted using the draft method (Appendix $A$ ) and deionized, distilled water as the extraction fluid. The extractions and analysis of the extracts were performed by Western Research Institute. These extractions were performed to determine if the acidic extraction fluids have any effect on the extractability of the two test materials used in the collaborative study.

The spray dryer waste and the composite mining waste were extracted in duplicate, and the resulting extracts were analyzed using ICP spectrometry (EPA Method 6010) (U.S. EPA 1990). The extracts were not digested prior to analysis.

\section{RESULTS AND DISCUSSION}

\section{Collaborative study Data}

The elemental concentrations determined in the collaborative study extracts, including those determined in the control extracts, were converted to microgram of element extracted per gram of sample and compensated, if necessary, for carry-over from one extraction step to the next. These calculations were performed by western Research Institute. Compensating the data for carry-over was performed as specified in section 10.9 of the method included as Appendix A. The data were corrected for carry-over if the difference between the corrected value and the uncorrected value was greater than 10\%. Differences less than $10 \%$ were not taken into account because those differences are less than the expected differences due to analytical error. The elemental concentrations determined in the analytical standards and the special analytical samples were also converted to units of microgram per gram.

As mentioned, the participants and teams of participants in the collaborative study were randomly designated as laboratory \#1, \#2, \#3, etc. Eight data sets were generated by the laboratories in the collaborative study. However, laboratories \#4 and \#6 did not follow the study 
specifications (see Appendix D), and as a result, the data generated by these laboratories were not used in the statistical evaluation to determine the precision of the extraction procedure. All of the participants analyzed their extracts using ICP spectrometry, and none of them, with the exception of laboratories \#7 and \#8, digested the extracts prior to analysis. The analytical methods used by the participants are listed in Appendix $D$, and collaborative study data summary sheets are included as Appendix E. To reduce the bulkiness of this report, the raw data generated by the laboratories are not presented; however, they are available from western Research Institute upon request.

\section{Statistical Malysis of the Collaborative study Data}

The data generated in the collaborative study can be divided into three categories. These are listed below.

1. Data from extraction of the test materials and analysis of the resulting extracts, which were used to determine the mean concentration for each element in each extract, $\bar{x}$, and the total standard deviation of the extraction procedure-plus-analysis of the extracts, $\mathbf{s}$.

2. Data from analysis of the analytical standards, which were used to determine the mean concentration for each level of analytical standard, $\vec{x}_{a}$, and the standard deviation for analysis of the analytical standards, $\mathbf{s}_{\mathrm{a}} \cdot$

3. Data from analysis of the special analytical samples, which were used to calculate the mean concentration for each type of special sample, $\bar{x}$, and the standard deviation, s.

ASTM Practice D2777, standard Practice for Determination of Precision and Bias of Applicable Methods of Committee D19 on Water (ASTM 1991) was used to evaluate the collaborative study data. The steps involved in the data analysis are listed below.

1. Tabulating the data.

2. Eliminating "outlier" laboratories (laboratories that were so consistently high or low that their results were unreasonable).

3. Eliminating any individual "outlier" data points.

4. Calculating multiple-laboratory total standard deviation (extraction procedure precision-plus-analytical precision).

5. Calculating single-operator total standard deviation (extraction procedure precision-plus-analytical precision).

6. Calculating multiple-laboratory analytical precision.

7. Calculating single-operator analytical precision.

8. Estimating multiple-laboratory extraction procedure precision.

9. Estimating single-operator extraction procedure precision. 
10. Calculating multiple-laboratory precision for analysis of the special analytical samples and evaluating the resulting data.

11. Comparing laboratory \#6 data (extractions continued through the weekend, Appendix D) versus data (final statistics) from laboratories \#1, 2, 3, 5, 7 , and 8 (extractions stopped over the weekend).

12. Comparing control data (water extracts) versus acidic extraction fluid data (final statistics).

13. Calculating bias for the analytical standards.

The steps outlined in ASTM Practice D2777 were applied to the data in the three categories described above. After calculations to eliminate "outlier" laboratories were performed, the mean concentration and standard deviation were calculated. The data were then evaluated for individual "outlier" data points at the 5\% significance level. If a data point was determined to be an "outlier", it was removed from the data set, and the mean and standard deviation were recalculated using the renaining data.

The statistical values that were calculated after "outlier" laboratory and individual "outlier" data points were eliminated from the data sets, are the final statistics for the collaborative study data. These values for the data resulting from extraction of the spray dryer waste and analysis of the resulting extracts are given in Tables 10-17. The final statistics for the data resulting from extraction of the composite mining waste and analysis of the resulting extracts are given in Tables 18-24. The final statistics for the data resulting from analysis of the spray dryer waste analytical standards are given in Tables 25-32, and these values for the data resulting from analysis of the composite mining waste analytical standards are given in Tables 33-39. The final statistics for the data resulting from analysis of the spray dryer waste special analytical samples are given in Tables 40-47, and these values for the data resulting from analysis of the composite mining waste special analytical samples are given in Tables 48-54.

Table 10. Final statistics for Aluminun in the Spray Dryer Waste sequential Batch Extracts, $\mu \mathrm{g} / \mathrm{g}$

\begin{tabular}{rccc}
\hline Extract & $\begin{array}{c}\text { Mean } \\
\text { Concentration } \\
\overline{\bar{x}}\end{array}$ & $\begin{array}{c}\text { standard } \\
\mathbf{s}\end{array}$ & $\begin{array}{c}\text { Deviation } \\
\text { Single-operator } \\
\text { standard Deviation } \\
\text { so }\end{array}$ \\
\hline 1 & 25.5 & 3.2 & 1.8 \\
2 & 69.3 & 10.4 & 2.7 \\
3 & 108 & 10 & 3 \\
4 & 128 & 10 & 3 \\
5 & 145 & 10 & 2 \\
6 & 160 & 12 & 2 \\
7 & 164 & 14 & 2 \\
8 & 167 & 15 & 2 \\
9 & 174 & 14 & 2 \\
10 & 183 & 12 &
\end{tabular}


Table 11. Final statistics for Barium in the spray Dryer waste sequential Batch Extracts, $\mu \mathrm{g} / \mathrm{g}$

\begin{tabular}{|c|c|c|c|}
\hline Extract & $\begin{array}{c}\text { Mean } \\
\text { Concentration } \\
\overline{\bar{x}}\end{array}$ & $\begin{array}{c}\text { Standard Deviation } \\
\mathrm{s}\end{array}$ & $\begin{array}{c}\text { single-operator } \\
\text { standard Deviation } \\
\mathbf{s}_{0}\end{array}$ \\
\hline 1 & 14 & 7 & 1 \\
\hline 2 & 62 & 34 & 1 \\
\hline 3 & 45 & 19 & 2 \\
\hline 4 & 38 & 12 & 7 \\
\hline 5 & 41.3 & 5.4 & 1.2 \\
\hline 6 & 32.1 & 1.1 & 0.7 \\
\hline 7 & 26.8 & 2.4 & 0.5 \\
\hline 8 & 24 & 4 & 0.3 \\
\hline 9 & 23.8 & 4.0 & 0.7 \\
\hline 10 & 19 & 3 & 1 \\
\hline
\end{tabular}

Table 12. Final statistics for Boron in the spray Dryer Waste sequential Batch Extracts, $\mu g / g$

\begin{tabular}{cccc}
\hline Extract & $\begin{array}{c}\text { Mean } \\
\text { Concentration } \\
\bar{x}\end{array}$ & $\begin{array}{c}\text { Standard Deviation } \\
\mathbf{s}\end{array}$ & $\begin{array}{c}\text { Single-operator } \\
\text { standard Deviation } \\
\mathbf{s}_{0}\end{array}$ \\
\hline 1 & 1.8 & 0.4 & 0.3 \\
2 & 1.7 & 0.8 & 0.5 \\
3 & 2.3 & 0.9 & 0.6 \\
4 & 2.6 & 0.5 & 0.4 \\
5 & 3.2 & 1.0 & 0.5 \\
6 & 3.6 & 0.8 & 0.3 \\
7 & 4.5 & 1.0 & 0.4 \\
8 & 4.4 & 0.8 & 0.4 \\
9 & 5.0 & 1.0 & 0.4 \\
\hline 10 & 5.3 & 1.3 & 0.5 \\
\hline
\end{tabular}


Table 13. Final statistics for Calcium in the spray Dryer Waste sequential Batch Extracts, $\mu \mathrm{g} / \mathrm{g}$

\begin{tabular}{cccc}
\hline Extract & $\begin{array}{c}\text { Mean } \\
\text { Concentration } \\
\bar{x}\end{array}$ & $\begin{array}{c}\text { Standard Deviation } \\
\mathbf{s}\end{array}$ & $\begin{array}{c}\text { Single-operator } \\
\text { Standard Deviation } \\
\text { so }\end{array}$ \\
\hline 1 & 3,755 & 261 & 154 \\
2 & 3,096 & 276 & 47 \\
3 & 2,795 & 111 & 55 \\
4 & 2,529 & 129 & 40 \\
5 & 2,301 & 79 & 37 \\
6 & 2,207 & 104 & 20 \\
7 & 2,119 & 79 & 32 \\
8 & 2,047 & 68 & 34 \\
9 & 1,960 & 107 & 55 \\
10 & 1,831 & 395 & 185 \\
\hline
\end{tabular}

Table 14. Final statistics for Chromium in the Spray Dryer waste sequential Batch Extracts, $\mu \mathrm{g} / \mathrm{g}$

\begin{tabular}{cccc}
\hline Extract & $\begin{array}{c}\text { Mean } \\
\text { Concentration } \\
\overline{\mathrm{x}}\end{array}$ & $\begin{array}{c}\text { Standard Deviation } \\
\mathbf{s}\end{array}$ & $\begin{array}{c}\text { Single-operator } \\
\text { standard Deviation } \\
\mathbf{s}_{0}\end{array}$ \\
\hline 1 & 2.8 & 0.3 & 0.2 \\
2 & 1.1 & 0.4 & $0.2^{\mathrm{a}}$ \\
\hline
\end{tabular}

a Values for the other extracts can not be calculated because the data sets contain "less-than" values. 
Table 15. Final statistics for silicon in the spray Dryer Waste sequential Batch Extracts, $\mu \mathrm{g} / \mathrm{g}$

\begin{tabular}{cccc}
\hline Extract & $\begin{array}{c}\text { Mean } \\
\text { Concentration } \\
\overline{\mathbf{x}}\end{array}$ & $\begin{array}{c}\text { Standard } \\
\mathbf{5}\end{array}$ & $\begin{array}{c}\text { Seviation } \\
\text { Single-operator } \\
\text { standard Deviation } \\
\text { s。 }\end{array}$ \\
\hline 1 & 181 & 15 & 6 \\
2 & 136 & 6 & 4 \\
3 & 106 & 8 & 2 \\
4 & 96 & 14 & 2 \\
5 & 97 & 8 & 3 \\
6 & 85.6 & 5.0 & 1.9 \\
7 & 80.1 & 6.8 & 2.6 \\
8 & 75.3 & 10.6 & 2.9 \\
9 & 69.1 & 9.3 & 4.4 \\
10 & 64.8 & 5.9 & 3.5 \\
\hline
\end{tabular}

Table 16. Final statistics for sodiun in the spray Dryer waste sequential Batch Extracts, $\mu \mathrm{g} / \mathrm{g}$

\begin{tabular}{cccc}
\hline Extract & $\begin{array}{c}\text { Mean } \\
\text { Concentration } \\
\overline{\mathbf{X}}\end{array}$ & $\begin{array}{c}\text { Standard } \\
\mathbf{s}\end{array}$ & $\begin{array}{c}\text { Deviation } \\
\text { single-operator } \\
\text { Standard Deviation } \\
\mathbf{s}_{0}\end{array}$ \\
\hline 1 & 3,515 & 342 & 50 \\
2 & 224 & 47 & 10 \\
3 & 100 & 11 & 5 \\
4 & 96 & 39 & 6 \\
5 & 98 & 24 & 5 \\
6 & 62 & 19 & 3 \\
7 & 50 & 11 & 2 \\
8 & 60 & 30 & 5 \\
9 & 60 & 15 & 4 \\
10 & 51 & 25 & 4 \\
\hline
\end{tabular}


Table 17. Final statistics for strontium in the spray Dryer Waste sequential Batch Extracts, $\mu \mathrm{g} / \mathrm{g}$

\begin{tabular}{rccc}
\hline Extract & $\begin{array}{c}\text { Mean } \\
\text { Concentration } \\
\overline{\mathrm{x}}\end{array}$ & $\begin{array}{c}\text { Standard } \\
\mathbf{s}\end{array}$ & $\begin{array}{c}\text { Deviation } \\
\text { Single-operator } \\
\text { standard Deviation } \\
\mathbf{S}_{0}\end{array}$ \\
\hline 1 & 214 & 18 & 6 \\
2 & 98 & 10 & 4 \\
3 & 61.9 & 5.3 & 4.1 \\
4 & 47.6 & 4.3 & 1.6 \\
5 & 42.7 & 3.4 & 1.5 \\
6 & 32.2 & 2.2 & 1.4 \\
7 & 26.8 & 1.7 & 1.2 \\
9 & 24.8 & 4.3 & 0.8 \\
10 & 25.4 & 3.1 & 3.3 \\
\hline
\end{tabular}

Table 18. Final statistics for Barium in the Composite Mining Waste Sequential Batch Extracts, $\mu \mathrm{g} / \mathrm{g}$

\begin{tabular}{rccc}
\hline Extract & $\begin{array}{c}\text { Mean } \\
\text { Concentration } \\
\overline{\mathrm{x}}\end{array}$ & $\begin{array}{c}\text { Standard } \\
\mathbf{s}\end{array}$ & $\begin{array}{c}\text { Seviation } \\
\text { Single-operator } \\
\text { standard Deviation } \\
\mathbf{s}_{0}\end{array}$ \\
\hline 1 & 1.9 & 0.3 & 0.2 \\
2 & 3.2 & 0.6 & 0.5 \\
3 & 2.3 & 0.3 & 0.2 \\
4 & 2.8 & 1.0 & 0.3 \\
5 & 6 & 2 & 1 \\
6 & 10 & 4 & 1 \\
7 & 15 & 1 & 0.2 \\
8 & $---^{\mathrm{a}}$ & $--^{\mathrm{a}}$ & $--^{\mathrm{a}}$ \\
9 & 12 & 2 & 1 \\
10 & 15 & 1 & 1 \\
\hline
\end{tabular}

a Value can not be calculated because the data set contains "less-than" values. 
Table 19. Final statistics for Calcium in the composite Mining Waste Sequential Batch Extracts, $\mu \mathrm{g} / \mathrm{g}$

\begin{tabular}{cccc}
\hline Extract & $\begin{array}{c}\text { Mean } \\
\text { Concentration } \\
\bar{x}\end{array}$ & $\begin{array}{c}\text { Standard } \\
\mathbf{s}\end{array}$ & $\begin{array}{c}\text { Single-operator } \\
\text { Standard } \\
\text { Deviation } \\
\text { s }\end{array}$ \\
\hline 1 & 1,815 & 238 & 139 \\
2 & 628 & 100 & 27 \\
3 & 448 & 64 & 22 \\
4 & 383 & 148 & 17 \\
5 & 268 & 16 & 14 \\
6 & 229 & 30 & 11 \\
7 & 228 & 26 & 9 \\
8 & 230 & 26 & 19 \\
9 & 222 & 20 & 19 \\
10 & 214 & 26 & 12 \\
\hline
\end{tabular}

Table 20. Final statistics for Lead in the composite Mining Waste sequential Batch Extracts, $\mu \mathrm{g} / \mathrm{g}$

\begin{tabular}{cccc}
\hline Extract & $\begin{array}{c}\text { Mean } \\
\text { Concentration } \\
\overline{\mathbf{x}}\end{array}$ & $\begin{array}{c}\text { Standard Deviation } \\
\mathbf{8}\end{array}$ & $\begin{array}{c}\text { Single-operator } \\
\text { standard Deviation } \\
\mathbf{s}_{0}\end{array}$ \\
\hline 1 & 63 & 8 & 3 \\
2 & 73 & 14 & $6^{\mathrm{a}}$ \\
\hline
\end{tabular}

a Values for the other extracts can not be calculated because the data sets contain "less-than" values. 
Table 21. Final statistics for Magnesium in the composite Mining Waste Sequential Batch Extracts, $\mu \mathrm{g} / \mathrm{g}$

\begin{tabular}{cccc}
\hline Extract & $\begin{array}{c}\text { Mean } \\
\text { Concentration } \\
\overline{\mathrm{x}}\end{array}$ & $\begin{array}{c}\text { Standard } \\
\text { Deviation }\end{array}$ & $\begin{array}{c}\text { Single-operator } \\
\text { Standard Deviation } \\
\text { so }\end{array}$ \\
\hline 1 & 107 & 12 & 7 \\
2 & 20 & 5 & 1 \\
3 & 12 & 2 & 1 \\
4 & 11 & 3 & 1 \\
5 & 10 & 2 & 1 \\
6 & 6 & 0.4 & 0.4 \\
7 & 5 & 0.5 & 0.3 \\
8 & 5 & 2 & 1 \\
9 & 6 & 1 & 1 \\
10 & 5 & 2 & 0.3 \\
\hline
\end{tabular}

Table 22. Final statistics for Manganese in the composite Mining Waste Sequential Batch Extracts, $\mu \mathrm{g} / \mathrm{g}$

\begin{tabular}{cccc}
\hline Extract & $\begin{array}{c}\text { Mean } \\
\text { Concentration } \\
\overline{\mathrm{x}}\end{array}$ & $\begin{array}{c}\text { Standard Deviation } \\
\mathrm{s}\end{array}$ & $\begin{array}{c}\text { single-Operator } \\
\text { standard Deviation } \\
\text { s。 }\end{array}$ \\
\hline 1 & 36.7 & 5.5 & 1.1 \\
2 & 9 & 2 & 1 \\
3 & 3.6 & 1.3 & 0.3 \\
4 & 1.8 & 1.5 & 0.2 \\
5 & 0.65 & 0.15 & $0.12^{\mathrm{a}}$ \\
\hline
\end{tabular}

a Values for the other extracts can not be calculated because the data sets contain "less-than" values. 
Table 23. Final statistics for silicon in the composite Mining Waste Sequential Batch Extracts, $\mu g / g$

\begin{tabular}{cccc}
\hline Extract & $\begin{array}{c}\text { Mean } \\
\text { Concentration } \\
\overline{\mathrm{x}}\end{array}$ & $\begin{array}{c}\text { Standard } \\
\mathbf{s}\end{array}$ & $\begin{array}{c}\text { Deviation } \\
\text { Single-operator } \\
\text { standard Deviation } \\
\text { S。 }\end{array}$ \\
\hline 1 & 55 & 21 & 2 \\
2 & 51 & 13 & 3 \\
3 & 35 & 6 & 3 \\
4 & 26 & 7 & 2 \\
5 & 20 & 3 & 2 \\
6 & 15 & 2 & 1 \\
7 & 14 & 2 & 1 \\
8 & 14 & 3 & 1 \\
9 & 14 & 2 & 1 \\
10 & 14 & 3 & 1 \\
\hline
\end{tabular}

Table 24. Final statistics for $\mathrm{zinc}$ in the Composite Mining Waste Sequential Batch Extracts, $\mu \mathrm{g} / \mathrm{g}$

\begin{tabular}{cccc}
\hline Extract & $\begin{array}{c}\text { Mean } \\
\text { Concentration } \\
\overline{\mathrm{x}}\end{array}$ & $\begin{array}{c}\text { Standard } \\
\mathbf{s}\end{array}$ & $\begin{array}{c}\text { Deviation } \\
\text { Single-operator } \\
\text { standard Deviation } \\
\text { S。 }\end{array}$ \\
\hline 1 & 297 & 35 & 31 \\
2 & 39 & 13 & 2 \\
3 & 13 & 10 & $1^{\text {a }}$ \\
\hline
\end{tabular}

a Values for the other extracts can not be calculated because the data sets contain "less-than" values. 
Table 25. Final statistics for Aluminum in the spray Dryer Waste Analytical Standards, $\mu \mathrm{g} / \mathrm{g}$

\begin{tabular}{lccc}
\hline Standard & $\begin{array}{c}\text { Mean } \\
\text { Concentration } \\
\overline{\mathbf{x}}_{\mathrm{a}}\end{array}$ & $\begin{array}{c}\text { Standard } \\
\text { Deviation }\end{array}$ & $\begin{array}{c}\text { Single-Operator } \\
\text { Standard Deviation } \\
\mathbf{s}_{0}\end{array}$ \\
\hline Low & 29 & 3 & 1 \\
Medium & 118 & 9 & 2 \\
High & 172 & 11 & 9 \\
\hline
\end{tabular}

Table 26. Final statistics for Barium in the Spray Dryer Waste Analytical standards, $\mu g / g$

\begin{tabular}{lccc}
\hline Standard & $\begin{array}{c}\text { Mean } \\
\text { Concentration } \\
\overline{\mathbf{x}}_{\mathrm{a}}\end{array}$ & $\begin{array}{c}\text { Standard Deviation } \\
\mathbf{s}_{\mathbf{a}}\end{array}$ & $\begin{array}{c}\text { Single-operator } \\
\text { standard Deviation } \\
\mathbf{s}_{0}\end{array}$ \\
\hline Low & 7 & 4 & 0.1 \\
Medium & 31 & 5 & 0.5 \\
High & 50 & 4 & 1 \\
\hline
\end{tabular}

Table 27. Final statistics for Boron in the spray Dryer waste Analytical standards, $\mu g / g$

\begin{tabular}{lccc}
\hline Standard & $\begin{array}{c}\text { Mean } \\
\text { Concentration } \\
\overline{\mathrm{x}}_{\mathrm{a}}\end{array}$ & $\begin{array}{c}\text { Standard Deviation } \\
\mathbf{s}_{\mathrm{a}}\end{array}$ & $\begin{array}{c}\text { Single-operator } \\
\text { Standard } \\
\text { Deviation } \\
\mathbf{s}_{\mathrm{o}}\end{array}$ \\
\hline Low & 4 & 3 & 0.2 \\
Medium & 5 & 3 & 0.3 \\
High & 9 & 3 & 0.4 \\
\hline
\end{tabular}


Table 28. Final statistics for Calcium in the spray Dryer Waste Analytical Standards, $\mu \mathrm{g} / \mathrm{g}$

\begin{tabular}{lccc}
\hline Standard & $\begin{array}{c}\text { Mean } \\
\text { Concentration } \\
\overline{\mathrm{K}}_{\mathrm{a}}\end{array}$ & standard Deviation & \multicolumn{2}{c}{$\begin{array}{c}\text { Single-Operator } \\
\text { Standard Deviation } \\
\mathbf{s}_{\mathrm{a}}\end{array}$} \\
\hline Low & 1,983 & 149 & 43 \\
Medium & 2,611 & 212 & 62 \\
High & 3,927 & 182 & 120 \\
\hline
\end{tabular}

Table 29. Final statistics for chromium in the spray Dryer waste High concentration Analytical standard, $\mu g / g$

\begin{tabular}{|c|c|c|c|}
\hline standard & $\begin{array}{c}\text { Mean } \\
\text { Concentration } \\
\mathrm{x}_{\mathrm{a}}\end{array}$ & $\begin{array}{c}\text { standard Deviation } \\
\mathbf{s}_{\mathbf{a}}\end{array}$ & $\begin{array}{c}\text { single-operator } \\
\text { standard Deviation } \\
s_{0}\end{array}$ \\
\hline High & 2.8 & 0.2 & $0.1^{a}$ \\
\hline
\end{tabular}

a Values for the other standards can not be calculated because the data sets contain "Iess-than" values.

rable 30. Final statistics for silicon in the spray Dryer Waste Analytical standards, $\mu g / 9$

\begin{tabular}{|c|c|c|c|}
\hline standard & $\begin{array}{c}\text { Mean } \\
\text { Concentration } \\
\bar{x}_{a}\end{array}$ & $\begin{array}{l}\text { standard Deviation } \\
\qquad s_{a}\end{array}$ & $\begin{array}{c}\text { single-operator } \\
\text { standard Deviation } \\
s_{0}\end{array}$ \\
\hline Low & 75 & 6 & 3 \\
\hline Medium & 107 & 8 & 3 \\
\hline High & 171 & 9 & 8 \\
\hline
\end{tabular}


Table 31. Final statistics for sodium in the spray Dryer Waste Analytical standards, $\mu g / \mathrm{g}$

\begin{tabular}{lccc}
\hline Standard & $\begin{array}{c}\text { Mean } \\
\text { Concentration } \\
\bar{x}_{\text {a }}\end{array}$ & standard Deviation & $\begin{array}{c}\text { Single-Operator } \\
\text { Standard Deviation } \\
\text { so }_{0}\end{array}$ \\
Low & 48 & 28 & 3 \\
Medium & 116 & 28 & 6 \\
High & 3,669 & 397 & 161 \\
\hline
\end{tabular}

Table 32. Final statistics for strontium in the spray Dryer Waste Analytical standards, $\mu \mathrm{g} / \mathrm{g}$

\begin{tabular}{lccc}
\hline Standard & $\begin{array}{c}\text { Mean } \\
\text { Concentration } \\
\bar{x}_{\mathrm{a}}\end{array}$ & $\begin{array}{c}\text { Standard Deviation } \\
\mathbf{s}_{\mathbf{a}}\end{array}$ & $\begin{array}{c}\text { Single-operator } \\
\text { standard Deviation } \\
\mathbf{s}_{0}\end{array}$ \\
Low & 19 & 1 & 0.2 \\
Medium & 46.7 & 2.7 & 1 \\
High & 188 & 10 & 3 \\
\hline
\end{tabular}

Table 33. Final statistics for Barium in the composite Mining Waste Analytical standards, $\mu g / g$

\begin{tabular}{lccc}
\hline Standard & $\begin{array}{c}\text { Mean } \\
\text { Concentration } \\
\bar{x}_{a}\end{array}$ & $\begin{array}{c}\text { Standard Deviation } \\
\mathbf{s}_{a}\end{array}$ & $\begin{array}{c}\text { Single-operator } \\
\text { standard } \\
\text { Deviation } \\
s_{0}\end{array}$ \\
\hline Low & 3 & 4 & 0.2 \\
Medium & 7 & 5 & 0.05 \\
High & 20 & 5 & 0.4 \\
\hline
\end{tabular}


Table 34. Final statistics for calcium in the composite Mining Waste Analytical standards, $\mu \mathrm{g} / \mathrm{g}$

\begin{tabular}{lccc}
\hline Standard & $\begin{array}{c}\text { Mean } \\
\text { Concentration } \\
\overline{\mathrm{x}}_{\mathrm{a}}\end{array}$ & $\begin{array}{c}\text { Standard Deviation } \\
\mathbf{s}_{\mathrm{a}}\end{array}$ & $\begin{array}{c}\text { single-Operator } \\
\text { standard Deviation } \\
\mathbf{s}_{0}\end{array}$ \\
\hline Low & 248 & 11 & 5 \\
Medium & 622 & 42 & 9 \\
High & 1,614 & 110 & 33 \\
\hline
\end{tabular}

Table 35. Final statistics for Lead in the Composite Mining Waste Analytical standards, $\mu \mathrm{g} / \mathrm{g}$

\begin{tabular}{lccc}
\hline Standard & $\begin{array}{c}\text { Mean } \\
\text { Concentration } \\
\bar{x}_{a}\end{array}$ & $\begin{array}{c}\text { standard Deviation } \\
\mathrm{s}_{\mathrm{a}}\end{array}$ & $\begin{array}{c}\text { Single-operator } \\
\text { Standard Deviation } \\
\mathbf{s}_{0}\end{array}$ \\
\hline Low & 5 & 1 & 0.5 \\
Medium & 31 & 1 & 1 \\
High & 91 & 4 & 3 \\
\hline
\end{tabular}

Table 36. Final statisties for Magnesium in the composite Mining Waste Analytical standards, $\mu g / g$

\begin{tabular}{lccc}
\hline Standard & $\begin{array}{c}\text { Mean } \\
\text { Concentration } \\
\overline{\mathbf{x}}_{\mathrm{a}}\end{array}$ & $\begin{array}{c}\text { Standard Deviation } \\
\mathbf{s}_{\mathbf{a}}\end{array}$ & $\begin{array}{c}\text { Single-operator } \\
\text { standard Deviation } \\
\mathbf{s}_{0}\end{array}$ \\
\hline Low & 6 & 1 & 0.2 \\
Medium & 14 & 1 & 0.4 \\
High & 102 & 5 & 1 \\
\hline
\end{tabular}


Table 37. Final statistics for Manganese in the Composite Mining Waste Mnalytical Standards, $\mu \mathrm{g} / \mathrm{g}$

\begin{tabular}{lccc}
\hline Standard & $\begin{array}{c}\text { Mean } \\
\text { Concentration } \\
\overline{\mathrm{x}}_{\mathrm{a}}\end{array}$ & $\begin{array}{c}\text { Standard Deviation } \\
\mathbf{s}_{\mathrm{a}}\end{array}$ & $\begin{array}{c}\text { Single-Operator } \\
\text { standard Deviation } \\
\mathbf{s}_{0}\end{array}$ \\
\hline Low & 1.0 & 0.1 & 0.03 \\
Medium & 6.1 & 0.3 & 0.2 \\
High & 39.9 & 1.7 & 0.5 \\
\hline
\end{tabular}

Table 38. Final statistics for silicon in the composite Mining Waste Analytical standards, $\mu g / g$

\begin{tabular}{lccc}
\hline Standard & $\begin{array}{c}\text { Mean } \\
\text { Concentration } \\
\overline{\mathbf{x}}_{\mathrm{a}}\end{array}$ & $\begin{array}{c}\text { Standard Deviation } \\
\mathbf{s}_{\mathrm{a}}\end{array}$ & $\begin{array}{c}\text { Single-operator } \\
\text { Standard Deviation } \\
\mathbf{s}_{\mathrm{o}}\end{array}$ \\
\hline Low & 13 & 2 & 1 \\
Medium & 34 & 3 & 1 \\
High & 44 & 3 & 1 \\
\hline
\end{tabular}

Table 39. Final statistics for zinc in the Composite Mining Waste Analytical standards, $\mu g / g$

\begin{tabular}{lccc}
\hline Standard & $\begin{array}{c}\text { Mean } \\
\text { Concentration } \\
\overline{\mathrm{x}}_{\mathrm{a}}\end{array}$ & $\begin{array}{c}\text { Standard Deviation } \\
\mathrm{s}_{\mathrm{a}}\end{array}$ & $\begin{array}{c}\text { Single-operator } \\
\text { Standard Deviation } \\
\mathbf{s}_{\mathrm{o}}\end{array}$ \\
\hline Low & 3.6 & 4.8 & 1.0 \\
Medium & 41.6 & 1.2 & 0.8 \\
High & 312 & 11 & 6 \\
\hline
\end{tabular}


Table 40. Final statistics for Aluminum in the spray Dryer waste special Analytical samples, $\mu g / g$

\begin{tabular}{lcc}
\hline Sample Type & $\begin{array}{c}\text { Mean concentration } \\
\overline{\mathbf{x}}\end{array}$ & Standard Deviation \\
\hline $\mathbf{s}$
\end{tabular}

Table 41. Final statistics for Barium in the spray Dryer waste special Analytical Samples, $\mu g / g$

\begin{tabular}{ccc}
\hline Sample Type & Mean $\begin{array}{c}\text { Concentration } \\
\overline{\mathrm{x}}\end{array}$ & $\begin{array}{c}\text { Standard Deviation } \\
\mathrm{s}\end{array}$ \\
\hline $\begin{array}{l}0.45-\mu \mathrm{m} \text { Filtrate } \\
\begin{array}{l}0.45-\mu \mathrm{m} \text { Filtrate } \\
\text { with Digestion }\end{array}\end{array}$ & 2.3 & 0.8 \\
$\begin{array}{l}0.8-\mu \mathrm{m} \text { Filtrate } \\
0.8-\mu \mathrm{m} \text { Filtrate }\end{array}$ & 75.3 & 6.7 \\
with Digestion & 2.6 & 1.2 \\
\hline
\end{tabular}

Table 42. Pinal statistics for Boron in the spray Dryer waste special Analytical Samples, $\mu g / g$

\begin{tabular}{lcc}
\hline Sample Type & $\begin{array}{c}\text { Mean } \\
\text { Concentration } \\
\bar{x}\end{array}$ & Standard Deviation \\
s
\end{tabular}


Table 43. Final statistics for Calcium in the spray Dryer Waste special Analytical samples, $\mu g / g$

\begin{tabular}{lcc}
\hline Sample Type & $\begin{array}{c}\text { Mean Concentration } \\
\overline{\mathrm{x}}\end{array}$ & $\begin{array}{c}\text { Standard Deviation } \\
\mathbf{s}\end{array}$ \\
\hline $0.45-\mu \mathrm{m}$ Filtrate & 4,077 & 542 \\
$\begin{array}{l}0.45-\mu \mathrm{m} \text { Filtrate } \\
\text { with Digestion }\end{array}$ & 3,592 & 553 \\
$0.8-\mu \mathrm{m}$ Filtrate & 3,981 & 534 \\
$\begin{array}{l}0.8-\mu \mathrm{m} \text { Filtrate } \\
\text { with Digestion }\end{array}$ & 3,582 & 481 \\
\hline
\end{tabular}

Table 44. Final statistics for chromium in the spray Dryer waste special Analytical Samples, $\mu g / g$

\begin{tabular}{lcc}
\hline Sample Type & Mean concentration & $\begin{array}{c}\text { standard Deviation } \\
\mathrm{s}\end{array}$ \\
\hline $0.45-\mu \mathrm{m}$ Filtrate & 2.7 & 0.2 \\
$\begin{array}{l}0.45-\mu \mathrm{m} \text { Filtrate } \\
\text { with Digestion }\end{array}$ & 2.6 & 0.1 \\
$0.8-\mu \mathrm{m}$ Filtrate & & \\
$\begin{array}{l}0.8-\mu \mathrm{m} \text { Filtrate } \\
\text { with Digestion }\end{array}$ & 2.6 & 0.3 \\
\hline
\end{tabular}

Table 45. Final statistics for silicon in the spray Dryer Waste special Analytical Samples, $\mu g / g$

\begin{tabular}{lcc}
\hline Sample Type & $\begin{array}{c}\text { Mean concentration } \\
\bar{x}\end{array}$ & Standard Deviation \\
s
\end{tabular}


Table 46. Final statistics for sodium in the spray Dryer waste special Analytical Samples, $\mu g / g$

\begin{tabular}{lcc}
\hline Sample Type & $\begin{array}{c}\text { Mean concentration } \\
\overline{\mathrm{x}}\end{array}$ & $\begin{array}{c}\text { Standard Deviation } \\
\mathrm{s}\end{array}$ \\
\hline $\begin{array}{l}0.45-\mu \mathrm{m} \text { Filtrate } \\
0.45-\mu \mathrm{m} \text { Filtrate } \\
\text { with Digestion }\end{array}$ & 3,510 & 509 \\
$0.8-\mu \mathrm{m}$ Filtrate & 3,132 & 475 \\
$\begin{array}{l}0.8-\mu \mathrm{m} \text { Filtrate } \\
\text { with Digestion }\end{array}$ & 3,471 & 510 \\
\hline
\end{tabular}

Table 47. Final statistics for strontium in the spray Dryer Waste special Analytical samples, $\mu \mathrm{g} / \mathrm{g}$

\begin{tabular}{lcc}
\hline \multicolumn{1}{c}{ Sample Type } & $\begin{array}{c}\text { Mean Concentration } \\
\overline{\mathrm{x}}\end{array}$ & Standard Deviation \\
\hline $0.45-\mu \mathrm{m}$ Filtrate & 209 & 8 \\
$\begin{array}{l}0.45-\mu \mathrm{m} \text { Filtrate } \\
\text { with Digestion }\end{array}$ & 199 & 2 \\
$\begin{array}{l}0.8-\mu \mathrm{m} \text { Filtrate } \\
0.8-\mu \mathrm{m} \text { Filtrate } \\
\text { with Digestion }\end{array}$ & 208 & 7 \\
\hline
\end{tabular}

Table 48. Final statistics for Barium in the Composite Mining Waste special Analytical samples, $\mu g / g$

\begin{tabular}{lcc}
\hline Sample Type & $\begin{array}{c}\text { Mean } \\
\text { Concentration }\end{array}$ & $\begin{array}{c}\text { standard Deviation } \\
\mathbf{s}\end{array}$ \\
\hline $\begin{array}{l}0.45-\mu m \text { Filtrate } \\
0.45-\mu m \text { Filtrate }\end{array}$ & 1.5 & 0.2 \\
with Digestion & 1.7 & 0.2 \\
$0.8-\mu$ m Filtrate & 1.6 & 0.2 \\
$\begin{array}{l}0.8-\mu \text { m Filtrate } \\
\text { with Digestion }\end{array}$ & 4.4 & 0.5 \\
\hline
\end{tabular}


Table 49. Final statistics for Calcium in the composite Mining Waste special Analytical samples, $\mu \mathrm{g} / \mathrm{g}$

\begin{tabular}{|c|c|c|}
\hline Sample Type & $\underset{\bar{x}}{\text { Mean Concentration }}$ & $\underset{\mathbb{S}}{\text { standard Deviation }}$ \\
\hline $0.45-\mu \mathrm{m}$ Filtrate & 1,652 & 209 \\
\hline 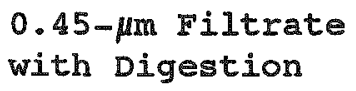 & 1,635 & 222 \\
\hline $0.8-\mu \mathrm{m}$ Filtrate & 1,712 & 231 \\
\hline $\begin{array}{l}0.8 \text { - } \mu \mathrm{m} \text { Filtrate } \\
\text { with Digestion }\end{array}$ & 1,592 & 221 \\
\hline
\end{tabular}

Table 50. Final statistics for Lead in the Composite Mining Waste special Analytica1 Samples, $\mu \mathrm{g} / \mathrm{g}$

\begin{tabular}{|c|c|c|c|}
\hline Sample Type & Mean Concentration & standard & $\begin{array}{l}\text { Deviation } \\
\mathbf{s}\end{array}$ \\
\hline $0.45-\mu \mathrm{m}$ Filtrate & 68 & & 6 \\
\hline $\begin{array}{l}0.48-\mu \mathrm{m} \text { Filtrate } \\
\text { with Digestion }\end{array}$ & 63 & & 5 \\
\hline $0.8-\mu \mathrm{m}$ Filtrate & 60 & & 6 \\
\hline $\begin{array}{l}0.8-\mu \mathrm{m} \text { Filtrate } \\
\text { with Digestion }\end{array}$ & 56 & & 5 \\
\hline
\end{tabular}

Table 51. Final statistics for Magnesium in the composite Mining Waste Special Analytical Samples, $\mu g / g$

\begin{tabular}{lcc}
\hline Sample Type & Mean Concentration & \multicolumn{2}{c}{ Standard Deviation } \\
s
\end{tabular}


Table 52. Final statistics for Manganese in the composite Mining Waste special Analytical Sanples, $\mu g / g$

\begin{tabular}{lcc}
\hline Sample Type & $\begin{array}{c}\text { Mean } \\
\text { Concentration }\end{array}$ & $\begin{array}{c}\text { standard Deviation } \\
\mathrm{s}\end{array}$ \\
\hline $0.45-\mu \mathrm{m}$ Filtrate & 36.2 & 3.6 \\
$\begin{array}{l}0.45-\mu \mathrm{m} \text { Filtrate } \\
\text { with Digestion }\end{array}$ & 34.2 & 3.1 \\
$\begin{array}{l}0.8-\mu \mathrm{m} \text { Filtrate } \\
\begin{array}{l}0.8-\mu \mathrm{m} \text { Filtrate } \\
\text { with Digestion }\end{array}\end{array}$ & 35.4 & 3.5 \\
\hline
\end{tabular}

Table 53. Final statistics for silicon in the Composite Mining Waste special Analytical Samples, $\mu \mathrm{g} / \mathrm{g}$

\begin{tabular}{|c|c|c|}
\hline Sample Type & $\underset{\overline{\mathrm{x}}}{\text { Mean Concentration }}$ & $\underset{\mathbf{s}}{\text { Standard Deviation }}$ \\
\hline $0.45-\mu \mathrm{m}$ Filtrate & 67 & 5 \\
\hline $\begin{array}{l}0.45-\mu \mathrm{m} \text { Filtrate } \\
\text { with Digestion }\end{array}$ & 63 & 8 \\
\hline $0.8-\mu \mathrm{m}$ Filtrate & 57 & 5 \\
\hline $\begin{array}{l}0.8-\mu \mathrm{m} \text { Filtrate } \\
\text { with Digestion }\end{array}$ & 49 & 8 \\
\hline
\end{tabular}

Table 54. Final statistics for zinc in the composite Mining Waste special Analytical Samples, $\mu \mathrm{g} / \mathrm{g}$

\begin{tabular}{ccc}
\hline Sample Type & $\begin{array}{c}\text { Mean } \\
\text { Concentration } \\
\overline{\mathrm{x}}\end{array}$ & $\begin{array}{c}\text { Standard Deviation } \\
\mathbf{s}\end{array}$ \\
\hline $0.45-\mu \mathrm{m}$ Filtrate & 298 & 30 \\
$\begin{array}{l}0.45-\mu \mathrm{m} \text { Filtrate } \\
\text { with Digestion }\end{array}$ & 298 & 38 \\
$0.8-\mu \mathrm{m}$ Filtrate & & 38 \\
$\begin{array}{l}0.8-\mu \mathrm{m} \text { Filtrate } \\
\text { with Digestion }\end{array}$ & 366 & 39 \\
\hline
\end{tabular}




\section{Estimated Precision of the Extraction Procedure}

The estimated multiple-laboratory precision of the extraction procedure, $s_{e}$, for each element of interest in the two test materials was calculated using the equation:

$$
s_{e}=\left[s^{2}-s_{a}^{2}\right]^{1 / 2}
$$

where $s$ is the standard deviation of the extraction procedure-plus-analysis of the extract, and $s_{a}$ is the standard deviation for analysis of the analytical standard containing the concentration of the specific element closest to its concentration in the extract. The estimated multiplelaboratory precisions of the extraction procedure for the elements of interest in the spray dryer waste are listed in Tables 55-62. The estimated multiple-laboratory precisions of the extraction procedure for the elements of interest in the composite mining waste are listed in Tables 63-69.

Table 55. Estimated Multiple-Laboratory precision of the sequential Batch Extraction Method Using Acidic Extraction Fluid in Extracting Aluminum from the Spray Dryer Waste, $\mu g / g$

\begin{tabular}{cccccc}
\hline & $\begin{array}{c}\text { Mean } \\
\text { Concentration } \\
\text { in Extract } \\
\text { Extract }\end{array}$ & $\begin{array}{c}\text { Mean } \\
\text { Concentration } \\
\text { in Analytical } \\
\text { Standard } \\
\overline{\mathbf{x}}_{\mathrm{a}}\end{array}$ & $\begin{array}{c}\text { Total } \\
\text { Precision } \\
\mathbf{s}\end{array}$ & $\begin{array}{c}\text { Analytical } \\
\text { Precision } \\
\mathbf{s}_{\mathbf{a}}\end{array}$ & $\begin{array}{c}\text { Estimated } \\
\text { Precision of } \\
\text { Extraction } \\
\text { Procedure } \\
\mathbf{s}_{\mathrm{e}}\end{array}$ \\
\hline 1 & 25.5 & 29 & 3.2 & 3 & 1 \\
2 & 69.3 & 29 & 10.4 & 3 & 10 \\
3 & 108 & 118 & 10 & 9 & 4 \\
4 & 128 & 118 & 10 & 9 & 4 \\
5 & 145 & 118 & 10 & 9 & 4 \\
6 & 160 & 172 & 12 & 11 & 5 \\
7 & 164 & 172 & 14 & 11 & 9 \\
8 & 167 & 172 & 15 & 11 & 9 \\
9 & 174 & 172 & 14 & 11 & 5 \\
10 & 183 & 172 & 12 & 11 & 9 \\
\hline
\end{tabular}


Table 56. Estimated Multiple-Laboratory Precision of the sequential Batch Extraction Method Using Acidic Extraction Fluid in Extracting Bariun from the Spray Dryer Waste, $\mu g / g$

\begin{tabular}{|c|c|c|c|c|c|}
\hline $\begin{array}{r}\text { con } \\
i \\
\text { Extract }\end{array}$ & $\begin{array}{l}\text { Mean } \\
\text { =entration } \\
\text { Extract } \\
\quad \overline{\mathbf{x}}\end{array}$ & $\begin{array}{c}\text { Mean } \\
\text { Concentration } \\
\text { in Analytical } \\
\text { standard } \\
\bar{x}_{a}\end{array}$ & $\begin{array}{c}\text { Total } \\
\text { Precision } \\
\mathbf{s}\end{array}$ & $\begin{array}{c}\text { Analytical } \\
\text { Precision } \\
\mathbf{s}_{\mathbf{a}}\end{array}$ & $\begin{array}{c}\text { Estimated } \\
\text { Precision of } \\
\text { Extraction } \\
\text { Procedure } \\
\mathbf{s}_{e}\end{array}$ \\
\hline 1 & 14 & 7 & 7 & 4 & 6 \\
\hline 2 & 62 & 50 & 34 & 4 & 34 \\
\hline 3 & 45 & 50 & 19 & 4 & 19 \\
\hline 4 & 38 & 31 & 12 & 5 & 11 \\
\hline 5 & 41.3 & 50 & 5.4 & 4 & 4 \\
\hline 6 & 32.1 & 31 & 1.1 & 5 & $--^{a}$ \\
\hline 7 & 26.8 & 31 & 2.4 & 5 & $--^{a}$ \\
\hline 8 & 24 & 31 & 4 & 5 & $--^{a}$ \\
\hline 9 & 23.8 & 31 & 4.0 & 5 & $--^{a}$ \\
\hline 10 & 19 & 31 & 3 & 5 & $-\ldots$ \\
\hline
\end{tabular}

a The multiple-laboratory standard deviation of the extraction procedure can not be determined.

Table 57. Estimated Multiple-Iaboratory Precision of the sequential Batch Extraction Method Using Acidic Extraction Fluid in Extracting Boron from the Spray Dryer Waste, $\mu \mathrm{g} / \mathrm{g}$

\begin{tabular}{|c|c|c|c|c|c|}
\hline Extract & $\begin{array}{l}\text { Mean } \\
\text { Concentration } \\
\text { in Extract } \\
\bar{x}\end{array}$ & $\begin{array}{c}\text { Mean } \\
\text { Concentration } \\
\text { in Analytical } \\
\text { standard } \\
\overline{\mathbf{x}}_{a}\end{array}$ & $\begin{array}{c}\text { Total } \\
\text { Precision } \\
\mathbf{s}\end{array}$ & $\begin{array}{c}\text { Analytical } \\
\text { Precision } \\
\mathrm{s}_{\mathrm{a}}\end{array}$ & $\begin{array}{c}\text { Estimated } \\
\text { Precision of } \\
\text { Extraction } \\
\text { Procedure } \\
\mathbf{s}_{e}\end{array}$ \\
\hline 1 & 1.8 & 4 & 0.4 & 3 & $--^{a}$ \\
\hline 2 & 1.7 & 4 & 0.8 & 3 & $--^{a}$ \\
\hline 3 & 2.3 & 4 & 0.9 & 3 & $--^{a}$ \\
\hline 4 & 2.6 & 4 & 0.5 & 3 & $-\ldots-^{a}$ \\
\hline 5 & 3.2 & 4 & 1.0 & 3 & $--^{a}$ \\
\hline 6 & 3.6 & 4 & 0.8 & 3 & $--^{a}$ \\
\hline 7 & 4.5 & 4 & 1.0 & 3 & $---^{a}$ \\
\hline 8 & 4.4 & 4 & 0.8 & 3 & $--^{a}$ \\
\hline 9 & 5.0 & 5 & 1.0 & 3 & $---^{\mathrm{a}}$ \\
\hline 10 & 5.3 & 5 & 1.3 & 3 & $---^{a}$ \\
\hline
\end{tabular}

a The multiple-laboratory standard deviation of the extraction procedure can not be determined. 
Table 58. Estimated Multiple-Laboratory Precision of the sequential Batch Extraction Method Using Acidic Extraction Fluid in Extracting Calciun from the Spray Dryer Waste, $\mu \mathrm{g} / \mathrm{g}$

\begin{tabular}{|c|c|c|c|c|c|}
\hline Extract & $\begin{array}{c}\text { Mean } \\
\text { Concentration } \\
\text { in Extract } \\
\bar{x}\end{array}$ & $\begin{array}{c}\text { Mean } \\
\text { Concentration } \\
\text { in Analytical } \\
\text { standard } \\
\overline{\mathrm{x}}_{\mathrm{a}}\end{array}$ & $\begin{array}{c}\text { Total } \\
\text { Precision } \\
\mathbf{s}\end{array}$ & $\begin{array}{c}\text { Analytical } \\
\text { Precision } \\
s_{a}\end{array}$ & $\begin{array}{c}\text { Estimated } \\
\text { Precision of } \\
\text { Extraction } \\
\text { Procedure } \\
\mathbf{s}_{e}\end{array}$ \\
\hline 1 & 3,755 & 3,927 & 261 & 182 & 187 \\
\hline 2 & 3,096 & 2,611 & 276 & 212 & 177 \\
\hline 3 & 2,795 & 2,611 & 111 & 212 & $--^{a}$ \\
\hline 4 & 2,529 & 2,611 & 129 & 212 & $--^{a}$ \\
\hline 5 & 2,301 & 2,611 & 79 & 212 & $--^{a}$ \\
\hline 6 & 2,207 & 1,983 & 104 & 149 & $--^{a}$ \\
\hline 7 & 2,119 & 1,983 & 79 & 149 & $--^{a}$ \\
\hline 8 & 2,047 & 1,983 & 68 & 149 & $--^{a}$ \\
\hline 9 & 1,960 & 1,983 & 107 & 149 & $--^{a}$ \\
\hline 10 & 1,831 & 1,983 & 395 & 149 & 366 \\
\hline
\end{tabular}

a The multiple-laboratory standard deviation of the extraction procedure can not be determined.

Table 59. Estimated Multiple-Laboratory Precision of the sequential Batch Extraction Method Using Acidic Extraction Fluid in Extracting Chromium from the Spray Dryer Waste, $\mu \mathrm{g} / \mathrm{g}$

\begin{tabular}{cccccc}
\hline & $\begin{array}{c}\text { Mean } \\
\text { Concentration } \\
\text { in Extract } \\
\overline{\mathrm{x}}\end{array}$ & $\begin{array}{c}\text { Moncentration } \\
\text { in Analytical } \\
\text { Standard } \\
\overline{\mathbf{x}}_{\mathrm{a}}\end{array}$ & $\begin{array}{c}\text { Total } \\
\text { Precision } \\
\mathbf{s}\end{array}$ & $\begin{array}{c}\text { Analytical } \\
\text { Precision } \\
\mathbf{s}_{\mathrm{a}}\end{array}$ & $\begin{array}{c}\text { Estimated } \\
\text { Precision of } \\
\text { Extraction } \\
\text { Procedure } \\
\mathrm{s}_{\mathrm{e}}\end{array}$ \\
\hline 1 & 2.8 & 2.8 & 0.3 & 0.2 & 0.2 \\
2 & 1.1 & 2.8 & 0.4 & 0.2 & $0.3^{\mathrm{a}}$ \\
\hline
\end{tabular}

a Values for the other extracts can not be calculated because the data sets contain "less-than" values. 
Table 60. Estimated Multiple-Laboratory Precision of the sequential Batch Extraction Method Using Acidic Extraction Fluid in Extracting silicon from the spray Dryer Waste, $\mu \mathrm{g} / \mathrm{g}$

\begin{tabular}{|c|c|c|c|c|c|}
\hline Extract & $\begin{array}{c}\text { Mean } \\
\text { Concentration } \\
\text { in Extract } \\
\overline{\mathrm{x}}\end{array}$ & $\begin{array}{c}\text { Mean } \\
\text { Concentration } \\
\text { in Analytical } \\
\text { standard } \\
\overline{\mathrm{x}}_{\mathrm{a}}\end{array}$ & $\begin{array}{c}\text { Total } \\
\text { Precision } \\
\mathbf{s}\end{array}$ & $\begin{array}{c}\text { Analytical } \\
\text { Precision } \\
\mathbf{s}_{\mathbf{a}}\end{array}$ & $\begin{array}{c}\text { Estimated } \\
\text { Precision of } \\
\text { Extraction } \\
\text { Procedure } \\
\mathrm{s}_{\mathrm{e}}\end{array}$ \\
\hline 1 & 181 & 171 & 15 & 9 & 12 \\
\hline 2 & 136 & 107 & 6 & 8 & $--^{2}$ \\
\hline 3 & 106 & 107 & 8 & 8 & 0 \\
\hline 4 & 96 & 107 & 14 & 8 & 11 \\
\hline 5 & 97 & 107 & 8 & 8 & 0 \\
\hline 6 & 85.6 & 75 & 5.0 & 6 & $--^{a}$ \\
\hline 7 & 80.1 & 75 & 6.8 & 6 & 3 \\
\hline 8 & 75.3 & 75 & 10.6 & 6 & 9 \\
\hline 9 & 69.1 & 75 & 9.3 & 6 & 7 \\
\hline 10 & 64.8 & 75 & 5.9 & 6 & $---^{a}$ \\
\hline
\end{tabular}

a The multiple-laboratory standard deviation of the extraction procedure can not be determined.

Table 61. Estimated Multiple-Laboratory Precision of the Sequential Batch Extraction Method Using Acidic Extraction Fluid in Extracting Sodium from the Spray Dryer Waste, $\mu \mathrm{g} / \mathrm{g}$

\begin{tabular}{|c|c|c|c|c|c|}
\hline Extract & $\begin{array}{c}\text { Mean } \\
\text { Concentration } \\
\text { in Extract } \\
t \\
t\end{array}$ & $\begin{array}{c}\text { Mean } \\
\text { Concentration } \\
\text { in Analytical } \\
\text { Standard } \\
\overline{\mathrm{x}}_{\mathrm{a}}\end{array}$ & $\begin{array}{c}\text { Total } \\
\text { Precision } \\
\mathbf{s}\end{array}$ & $\begin{array}{c}\text { Analytical } \\
\text { Precision } \\
\mathrm{s}_{\mathrm{a}}\end{array}$ & $\begin{array}{c}\text { Estimated } \\
\text { Precision of } \\
\text { Extraction } \\
\text { Procedure } \\
\mathbf{s}_{\mathrm{e}}\end{array}$ \\
\hline 1 & 3,515 & 3,669 & 342 & 397 & $---^{a}$ \\
\hline 2 & 224 & 116 & 47 & 28 & 38 \\
\hline 3 & 100 & 116 & 11 & 28 & $--^{a}$ \\
\hline 4 & 96 & 116 & 39 & 28 & 27 \\
\hline 5 & 98 & 116 & 24 & 28 & $--^{a}$ \\
\hline 6 & 62 & 48 & 19 & 28 & $---^{a}$ \\
\hline 7 & 50 & 48 & 11 & 28 & $--^{a}$ \\
\hline 8 & 60 & 48 & 30 & 28 & 11 \\
\hline 9 & 60 & 48 & 15 & 28 & $---^{a}$ \\
\hline 10 & 51 & 48 & 25 & 28 & $--^{a}$ \\
\hline
\end{tabular}

a The multiple-laboratory standard deviation of the extraction procedure can not be determined. 
Table 62. Estimated Multiple-Laboratory Precision of the sequential Batch Extraction Method Using Acidic Extraction Fluid in Extracting strontiun from the spray Dryer Waste, $\mu g / g$

\begin{tabular}{rccccc}
\hline & $\begin{array}{c}\text { Mean } \\
\text { Concentration } \\
\text { in Extract } \\
\text { Extract }\end{array}$ & $\begin{array}{c}\text { Mean } \\
\text { Concentration } \\
\text { in Analytical } \\
\text { Standard } \\
\overline{\bar{x}}_{\mathrm{a}}\end{array}$ & $\begin{array}{c}\text { Total } \\
\text { Precision } \\
\mathbf{s}\end{array}$ & $\begin{array}{c}\text { Analytical } \\
\text { Precision } \\
\mathbf{s}_{\mathrm{a}}\end{array}$ & $\begin{array}{c}\text { Estimated } \\
\text { Precision of } \\
\text { Extraction } \\
\text { Procedure } \\
\mathbf{s}_{\mathrm{e}}\end{array}$ \\
\hline 1 & 214 & 188 & 18 & 10 & 15 \\
2 & 98 & 46.7 & 10 & 2.7 & 10 \\
3 & 61.9 & 46.7 & 5.3 & 2.7 & 4.6 \\
4 & 47.6 & 46.7 & 4.3 & 2.7 & 3.3 \\
5 & 42.7 & 46.7 & 3.4 & 2.7 & 2.1 \\
6 & 32.2 & 19 & 2.2 & 1 & 2 \\
7 & 26.8 & 19 & 1.7 & 1 & 4 \\
8 & 24.8 & 19 & 4.3 & 1 & 3 \\
9 & 25.4 & 19 & 3.1 & 1 & 0 \\
10 & 19 & 19 & 1 & 1 & 4 \\
\hline
\end{tabular}

Table 63. Estimated Multiple-Laboratory Precision of the sequential Batch Extraction Method Using Acidic Extraction Fluid in Extracting Barium from the Composite Mining Waste, $\mu \mathrm{g} / \mathrm{g}$

\begin{tabular}{|c|c|c|c|c|c|}
\hline Extract & $\begin{array}{c}\text { Mean } \\
\text { Concentration } \\
\text { in Extract } \\
\overline{\mathrm{x}}\end{array}$ & $\begin{array}{c}\text { Mean } \\
\text { Concentration } \\
\text { in Analytical } \\
\text { standard } \\
\overline{\mathrm{x}}_{\mathrm{a}}\end{array}$ & $\begin{array}{c}\text { Total } \\
\text { Precision } \\
\text { s }\end{array}$ & $\begin{array}{c}\text { Analytical } \\
\text { Precision } \\
s_{a}\end{array}$ & $\begin{array}{c}\text { Estinated } \\
\text { Precision of } \\
\text { Extraction } \\
\text { Procedure } \\
\mathrm{s}_{\mathrm{e}}\end{array}$ \\
\hline 1 & 1.9 & 3 & 0.3 & 4 & $--^{a}$ \\
\hline 2 & 3.2 & 3 & 0.6 & 4 & $--^{a}$ \\
\hline 3 & 2.3 & 3 & 0.3 & 4 & $--^{a}$ \\
\hline 4 & 2.8 & 3 & 1.0 & 4 & $--^{a}$ \\
\hline 5 & 6 & 7 & 2 & 5 & $--^{a}$ \\
\hline 6 & 10 & 7 & 4 & 5 & $--^{a}$ \\
\hline 7 & 15 & 20 & 1 & 5 & $--^{a}$ \\
\hline 8 & $------\infty$ & $-\infty$ & -- & -0 & $---b$ \\
\hline 9 & 12 & 7 & 2 & 5 & $--^{a}$ \\
\hline 10 & 15 & 20 & 1 & 5 & $--^{a}$ \\
\hline
\end{tabular}

a The multiple-laboratory standard deviation of the extraction procedure can not be determined.

b Calculations can not be performed because the data sets contain "lessthan" values. 
Table 64. Estimated Multiple-Laboratory Precision of the sequential Batch Extraction Method Using Acidic Extraction Fluid in Extracting Calcium from the Composite Mining Waste, $\mu \mathrm{g} / \mathrm{g}$

\begin{tabular}{cccccc}
\hline & $\begin{array}{c}\text { Mean } \\
\text { Concentration } \\
\text { in Extract } \\
\text { Extract }\end{array}$ & $\begin{array}{c}\text { Meancentration } \\
\text { in Analytical } \\
\text { Standard } \\
\overline{\mathbf{x}}_{2}\end{array}$ & $\begin{array}{c}\text { Total } \\
\text { Precision }\end{array}$ & $\begin{array}{c}\text { Analytical } \\
\text { Precision } \\
\mathbf{s}_{2}\end{array}$ & $\begin{array}{c}\text { Estimated } \\
\text { Precision of } \\
\text { Extraction } \\
\text { Procedure } \\
\mathbf{s}_{\mathrm{e}}\end{array}$ \\
\hline 1 & 1,815 & 1,614 & 238 & 110 & 211 \\
2 & 628 & 622 & 100 & 42 & 91 \\
3 & 448 & 622 & 64 & 42 & 48 \\
4 & 383 & 248 & 148 & 11 & 148 \\
5 & 268 & 248 & 16 & 11 & 12 \\
6 & 229 & 248 & 30 & 11 & 28 \\
7 & 228 & 248 & 26 & 11 & 24 \\
8 & 230 & 248 & 26 & 11 & 24 \\
9 & 222 & 248 & 20 & 11 & 17 \\
10 & 214 & 248 & 26 & 11 & 24 \\
\hline
\end{tabular}

Table 65. Estimated Multiple-Laboratory Precision of the Sequential Batch Extraction Method Using Acidic Extraction Fluid in Extracting Lead from the Composite Mining Waste, $\mu \mathrm{g} / \mathrm{g}$

\begin{tabular}{cccccc}
\hline & $\begin{array}{c}\text { Mean } \\
\text { Concentration } \\
\text { in Extract } \\
\overline{\mathrm{x}}\end{array}$ & $\begin{array}{c}\text { Concentration } \\
\text { in Analytical } \\
\text { Standard } \\
\overline{\mathrm{x}}_{\mathrm{a}}\end{array}$ & $\begin{array}{c}\text { Total } \\
\text { Precision } \\
\mathrm{s}\end{array}$ & $\begin{array}{c}\text { Analytical } \\
\text { Precision } \\
\overline{\mathrm{x}}_{\mathrm{a}}\end{array}$ & $\begin{array}{c}\text { Estimated } \\
\text { Precision of } \\
\text { Extraction } \\
\text { Procedure } \\
\mathbf{s}_{\mathbf{e}}\end{array}$ \\
\hline 1 & 63 & 91 & 8 & 4 & 7 \\
2 & 73 & 91 & 14 & 4 & $13^{\mathrm{a}}$ \\
\hline
\end{tabular}

a Values for the other extracts can not be calculated because the data sets contain "less-than" values. 
Table 66. Estimated Multiple-Laboratory precision of the Sequential Batch Extraction Method Using Acidic Extraction Fiuid in Extracting Magnesium from the Composite Mining Waste, $\mu \mathrm{g} / \mathrm{g}$

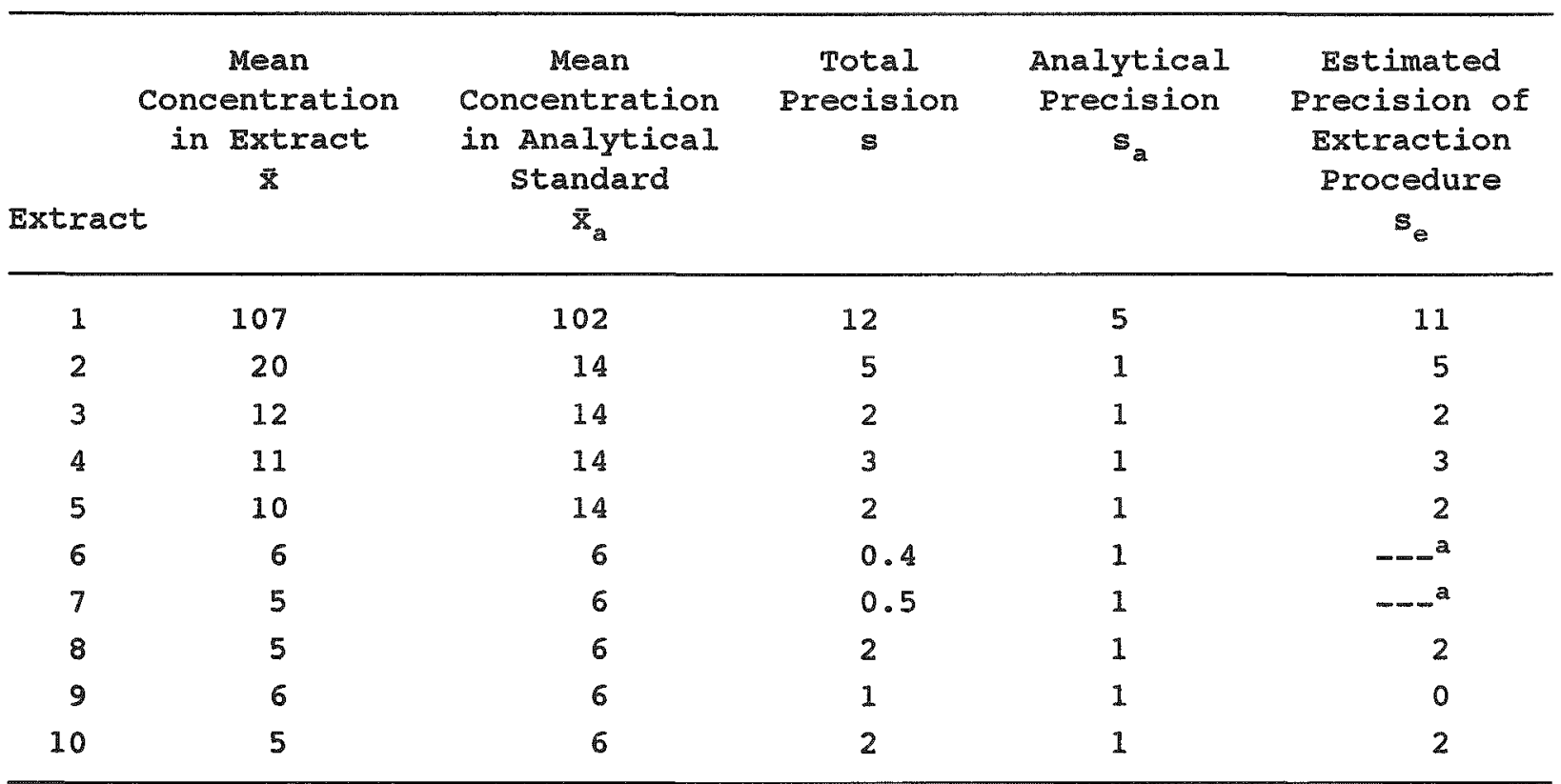

a The multiple-laboratory standard deviation of the extraction procedure can not be determined.

Table 67. Estimated Multiple-Laboratory Precision of the sequential Batch Extraction Method using Acidic Extraction Fluid in Extracting Manganese from the Composite Mining Waste, $\mu g / g$

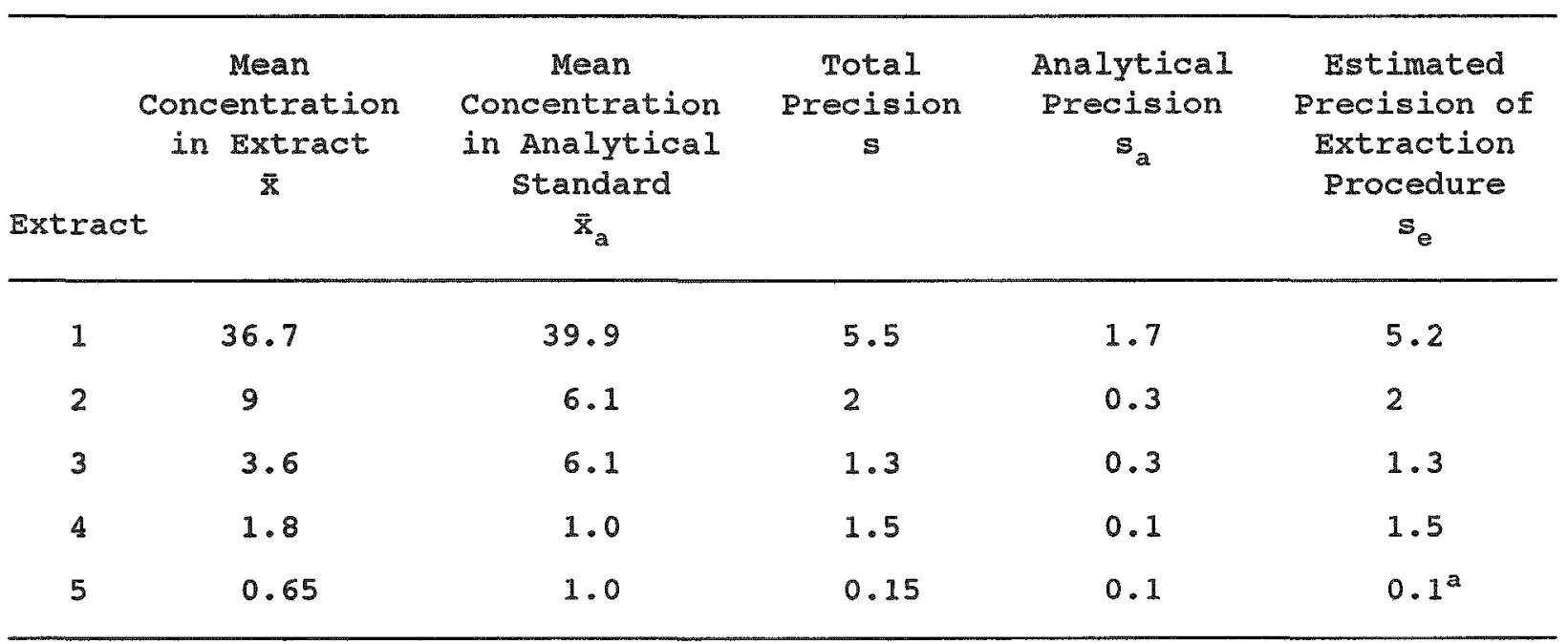

a Values for the other extracts can not be calculated because the data sets contain "less-than" values. 
Table 68. Estimated Multiple-Laboratory Precision of the sequential Batch Extraction Method Using Acidic Extraction Fluid in Extracting silicon from the Composite Mining Waste, $\mu \mathrm{g} / \mathrm{g}$

\begin{tabular}{|c|c|c|c|c|c|}
\hline Extract & $\begin{array}{c}\text { Mean } \\
\text { Concentration } \\
\text { in Extract } \\
\bar{t}\end{array}$ & $\begin{array}{c}\text { Mean } \\
\text { Concentration } \\
\text { in Analytical } \\
\text { standard } \\
\bar{x}_{a}\end{array}$ & $\begin{array}{c}\text { Total } \\
\text { Precision } \\
\mathbf{S}\end{array}$ & $\begin{array}{c}\text { Analytical } \\
\text { Precision } \\
\mathbf{s}_{\mathbf{a}}\end{array}$ & $\begin{array}{c}\text { Estimated } \\
\text { Precision of } \\
\text { Extraction } \\
\text { Procedure } \\
\mathbf{s}_{\mathrm{e}}\end{array}$ \\
\hline 1 & 55 & 44 & 21 & 3 & 21 \\
\hline 2 & 51 & 44 & 13 & 3 & 13 \\
\hline 3 & 35 & 34 & 6 & 3 & 5 \\
\hline 4 & 26 & 34 & 7 & 3 & 6 \\
\hline 5 & 20 & 13 & 3 & 2 & 2 \\
\hline 6 & 15 & 13 & 2 & 2 & 0 \\
\hline 7 & 14 & 13 & 2 & 2 & 0 \\
\hline 8 & 14 & 13 & 3 & 2 & 2 \\
\hline 9 & 14 & 13 & 2 & 2 & 0 \\
\hline 10 & 14 & 13 & 3 & 2 & 2 \\
\hline
\end{tabular}

Table 69. Estimated Multiple-Laboratory Precision of the sequential Batch Extraction Method Using Acidic Extraction Fluid in Extracting zinc from the Composite Mining Waste, $\mu \mathrm{g} / \mathrm{g}$

\begin{tabular}{cccccc}
\hline & $\begin{array}{c}\text { Mean } \\
\text { Concentration } \\
\text { in Extract } \\
\overline{\mathrm{x}}\end{array}$ & $\begin{array}{c}\text { Concentration } \\
\text { in Analytical } \\
\text { Standard } \\
\overline{\mathbf{x}}_{\mathrm{a}}\end{array}$ & $\begin{array}{c}\text { Total } \\
\text { Precision } \\
\mathbf{s}\end{array}$ & $\begin{array}{c}\text { Analytical } \\
\text { Precision } \\
\mathbf{s}_{\mathbf{a}}\end{array}$ & $\begin{array}{c}\text { Estimated } \\
\text { Precision of } \\
\text { Extraction } \\
\text { Procedure } \\
\mathbf{s}_{\mathbf{e}}\end{array}$ \\
\hline 1 & 297 & 312 & 35 & 11 & 33 \\
2 & 39 & 41.6 & 13 & 1.2 & 13 \\
3 & 13 & 3.6 & 10 & 4.8 & 9 \\
\hline
\end{tabular}

a Values for the other extracts can not be calculated because the data sets contain "less-than" values. 
The estimated single-operator precision of the extraction procedure, $\mathbf{s}_{o e}$ for each element was calculated using the equation:

$$
s_{o e}=\left[s_{0}^{2}-s_{o a}{ }^{2}\right]^{1 / 2} \text {, }
$$

where $s_{0}$ is the single-operator standard deviation of the extraction procedure-plus-analysis of the extract, and $s_{o a}$ is the single-operator standard deviation for analysis of the analytical standard containing the concentration of the specific element closest to its concentration in the extract. The estimated single-operator precisions of the extraction procedure for the elements of interest in the spray dryer waste are listed in Tables 70-77. The estimated single-operator precisions of the extraction procedure for the elements of interest in the composite mining waste are listed in Tables 78-84.

Table 70. Estimated single-operator Precision of the sequential Batch Extraction Method Using Acidic Extraction Fluid in Extracting Aluminum from the spray Dryer Waste, $\mu \mathrm{g} / \mathrm{g}$

\begin{tabular}{|c|c|c|c|c|c|}
\hline $\begin{array}{r}\text { con } \\
\text { is } \\
\text { Extract }\end{array}$ & $\begin{array}{l}\text { Mean } \\
\text { centration } \\
\text { Extract } \\
\bar{x}\end{array}$ & $\begin{array}{c}\text { Mean } \\
\text { Concentration } \\
\text { in Analytical } \\
\text { standard } \\
\overline{\mathrm{x}}_{\mathrm{a}}\end{array}$ & $\begin{array}{c}\text { Single- } \\
\text { Operator } \\
\text { Precision } \\
\text { s。 }\end{array}$ & $\begin{array}{c}\text { single- } \\
\text { operator } \\
\text { Analytical } \\
\text { Precision } \\
\text { soa }\end{array}$ & $\begin{array}{c}\text { Estimated Single- } \\
\text { operator Precision } \\
\text { of the Extraction } \\
\text { Procedure } \\
\text { soe }_{\text {of }}\end{array}$ \\
\hline 1 & 25.5 & 29 & 1.8 & 1 & 1 \\
\hline 2 & 69.3 & 29 & 2.7 & 1 & 2 \\
\hline 3 & 108 & 118 & 3 & 2 & 2 \\
\hline 4 & 128 & 118 & 3 & 2 & 2 \\
\hline 5 & 145 & 118 & 2 & 2 & 0 \\
\hline 6 & 160 & 172 & 2 & 9 & $--^{a}$ \\
\hline 7 & 164 & 172 & 2 & 9 & $--^{a}$ \\
\hline 8 & 167 & 172 & 2 & 9 & $--^{a}$ \\
\hline 9 & 174 & 172 & 2 & 9 & $--^{a}$ \\
\hline 10 & 183 & 172 & 2 & 9 & $--^{a}$ \\
\hline
\end{tabular}

a The single-operator standard deviation of the extraction procedure can not be determined. 
Table 71. Estimated single-operator Precision of the sequential Batch Extraction Method Using Mcidic Extraction Fluid in Extracting Barium from the Spray Dryer Waste, $\mu \mathrm{g} / \mathrm{g}$

\begin{tabular}{|c|c|c|c|c|c|}
\hline $\begin{array}{r}\text { Con } \\
\text { in } \\
\text { Extract }\end{array}$ & $\begin{array}{l}\text { Mean } \\
\text { entration } \\
\text { Extract } \\
\bar{x}\end{array}$ & $\begin{array}{c}\text { Mean } \\
\text { Concentration } \\
\text { in Analytical } \\
\text { standard } \\
\bar{x}_{a}\end{array}$ & $\begin{array}{c}\text { single- } \\
\text { operator } \\
\text { Precision } \\
\text { s. }_{0}\end{array}$ & $\begin{array}{c}\text { single- } \\
\text { Operator } \\
\text { Analytical } \\
\text { Precision } \\
\text { Soa }\end{array}$ & $\begin{array}{c}\text { Estimated single- } \\
\text { Operator Precision } \\
\text { of the Extraction } \\
\text { Procedure } \\
\text { soe }^{\text {o }}\end{array}$ \\
\hline 1 & 14 & 7 & 1 & 0.1 & 1 \\
\hline 2 & 62 & 50 & 1 & 1 & 0 \\
\hline 3 & 45 & 50 & 2 & 1 & 2 \\
\hline 4 & 38 & 31 & 7 & 0.5 & 7 \\
\hline 5 & 41.3 & 50 & 1.2 & 1 & 1 \\
\hline 6 & 32.1 & 31 & 0.7 & 0.5 & 0.5 \\
\hline 7 & 26.8 & 31 & 0.5 & 0.5 & 0 \\
\hline 8 & 24 & 31 & 0.3 & 0.5 & $--^{a}$ \\
\hline 9 & 23.8 & 31 & 0.7 & 0.5 & 0.5 \\
\hline 10 & 19 & 31 & 1 & 0.5 & 1 \\
\hline
\end{tabular}

a The single-operator standard deviation of the extraction procedure can not be determined.

Table 72. Estimated single-operator Precision of the sequential Batch Extraction Method Using Acidic Extraction Fluid in Extracting Boron from the spray Dryer Waste, $\mu \mathrm{g} / \mathrm{g}$

\begin{tabular}{|c|c|c|c|c|c|}
\hline $\begin{array}{r}\text { Cone } \\
\text { in } \\
\text { Extract }\end{array}$ & $\begin{array}{l}\text { Mean } \\
\text { Entration } \\
\text { Extract } \\
\overline{\bar{x}}\end{array}$ & $\begin{array}{c}\text { Mean } \\
\text { Concentration } \\
\text { in Analytical } \\
\text { standard } \\
\bar{x}_{\mathrm{a}}\end{array}$ & $\begin{array}{c}\text { single- } \\
\text { operator } \\
\text { Precision } \\
\text { s. }\end{array}$ & $\begin{array}{c}\text { single- } \\
\text { Operator } \\
\text { Analytical } \\
\text { Precision } \\
\text { soa }\end{array}$ & $\begin{array}{c}\text { Estimated single- } \\
\text { operator Precision } \\
\text { of the Extraction } \\
\text { Procedure } \\
\text { soe }^{\text {of }}\end{array}$ \\
\hline 1 & 1.8 & 4 & 0.3 & 0.2 & 0.2 \\
\hline 2 & 1.7 & 4 & 0.5 & 0.2 & 0.5 \\
\hline 3 & 2.3 & 4 & 0.6 & 0.2 & 0.6 \\
\hline 4 & 2.6 & 4 & 0.4 & 0.2 & 0.3 \\
\hline 5 & 3.2 & 4 & 0.5 & 0.2 & 0.5 \\
\hline 6 & 3.6 & 4 & 0.3 & 0.2 & 0.2 \\
\hline 7 & 4.5 & 4 & 0.4 & 0.2 & 0.3 \\
\hline 8 & 4.4 & 4 & 0.4 & 0.2 & 0.3 \\
\hline 9 & 5.0 & 5 & 0.4 & 0.3 & 0.3 \\
\hline 10 & 5.3 & 5 & 0.5 & 0.3 & 0.4 \\
\hline
\end{tabular}


Table 73. Estimated single-operator Precision of the sequential Batch Extraction Method Using Acidic Extraction Fluid in Extracting Calcium from the Spray Dryer Waste, $\mu g / g$

\begin{tabular}{|c|c|c|c|c|c|}
\hline $\begin{array}{r}\text { Con } \\
\text { in } \\
\text { Extract }\end{array}$ & $\begin{array}{l}\text { Mean } \\
\text { oncentration } \\
\text { in Extract } \\
\qquad \bar{x}\end{array}$ & $\begin{array}{c}\text { Mean } \\
\text { Concentration } \\
\text { in Analytical } \\
\text { standard } \\
\overline{\mathrm{x}}_{\mathrm{a}}\end{array}$ & $\begin{array}{c}\text { Single- } \\
\text { Operator } \\
\text { Precision } \\
\text { s. }\end{array}$ & $\begin{array}{c}\text { single- } \\
\text { operator } \\
\text { Analytical } \\
\text { Precision } \\
\text { soa }\end{array}$ & $\begin{array}{c}\text { Estimated Single- } \\
\text { operator Precision } \\
\text { of the Extraction } \\
\text { Procedure } \\
\text { soe }_{\text {o }}\end{array}$ \\
\hline 1 & 3,755 & 3,927 & 154 & 120 & 97 \\
\hline 2 & 3,096 & 2,611 & 47 & 62 & $--^{a}$ \\
\hline 3 & 2,795 & 2,611 & 55 & 62 & $---^{a}$ \\
\hline 4 & 2,529 & 2,611 & 40 & 62 & $--^{a}$ \\
\hline 5 & 2,301 & 2,611 & 37 & 62 & $---^{a}$ \\
\hline 6 & 2,207 & 1,983 & 20 & 43 & $---^{2}$ \\
\hline 7 & 2,119 & 1,983 & 32 & 43 & $--^{a}$ \\
\hline 8 & 2,047 & 1,983 & 34 & 43 & $--^{\mathbf{a}}$ \\
\hline 9 & 1,960 & 1,983 & 55 & 43 & 34 \\
\hline 10 & 1,831 & 1,983 & 185 & 43 & 180 \\
\hline
\end{tabular}

a The single-operator standard deviation of the extraction procedure can not be determined.

Table 74. Estimated single-operator Precision of the sequential Batch Extraction Method Using Acidic Extraction Fluid in Extracting Chromium from the Spray Dryer Waste, $\mu \mathrm{g} / \mathrm{g}$

\begin{tabular}{|c|c|c|c|c|c|}
\hline $\begin{array}{r}\text { con } \\
\text { i } \\
\text { Extract }\end{array}$ & $\begin{array}{l}\text { Mean } \\
\text { gentration } \\
\text { Extract } \\
\quad \bar{x}\end{array}$ & $\begin{array}{c}\text { Mean } \\
\text { Concentration } \\
\text { in Analytical } \\
\text { standard } \\
\overline{\mathbf{x}}_{\mathrm{a}}\end{array}$ & $\begin{array}{c}\text { Single- } \\
\text { Operator } \\
\text { Precision } \\
\text { s. }\end{array}$ & $\begin{array}{c}\text { single- } \\
\text { operator } \\
\text { Analytical } \\
\text { Precision } \\
\text { soa }\end{array}$ & $\begin{array}{c}\text { Estimated Single- } \\
\text { operator Precision } \\
\text { of the Extraction } \\
\text { Procedure } \\
\text { soe }\end{array}$ \\
\hline 1 & 2.8 & 2.8 & 0.2 & 0.1 & 0.2 \\
\hline 2 & 1.1 & 2.8 & 0.2 & 0.1 & $0.2^{\mathrm{a}}$ \\
\hline
\end{tabular}

a Values for the other extracts can not be calculated because the data sets contain "less-than" values. 
Table 75. Estimated single-operator Precision of the sequential Batch Extraction Method Using Acidic Extraction Fluid in Extracting silicon from the spray Dryer waste, $\mu \mathrm{g} / \mathrm{g}$

\begin{tabular}{|c|c|c|c|c|c|}
\hline $\begin{array}{r}\text { con } \\
\text { ir } \\
\text { Extract }\end{array}$ & $\begin{array}{c}\text { Mean } \\
\text { oncentration } \\
\text { in Extract } \\
\qquad \bar{x}\end{array}$ & $\begin{array}{c}\text { Mean } \\
\text { Concentration } \\
\text { in Analytical } \\
\text { standard } \\
\overline{\mathrm{x}}_{\mathrm{a}}\end{array}$ & $\begin{array}{c}\text { single- } \\
\text { Operator } \\
\text { Precision } \\
\text { s. }\end{array}$ & $\begin{array}{c}\text { single- } \\
\text { operator } \\
\text { Analytical } \\
\text { Precision } \\
\text { soa }\end{array}$ & $\begin{array}{c}\text { Estimated single- } \\
\text { operator Precision } \\
\text { of the Extraction } \\
\text { Procedure } \\
\text { soe }\end{array}$ \\
\hline 1 & 181 & 171 & 6 & 8 & $---^{a}$ \\
\hline 2 & 136 & 107 & 4 & 3 & 3 \\
\hline 3 & 106 & 107 & 2 & 3 & $--^{a}$ \\
\hline 4 & 96 & 107 & 2 & 3 & $--^{a}$ \\
\hline 5 & 97 & 107 & 3 & 3 & 0 \\
\hline 6 & 85.6 & 75 & 1.9 & 3 & $--^{a}$ \\
\hline 7 & 80.1 & 75 & 2.6 & 3 & $--^{a}$ \\
\hline 8 & 75.3 & 75 & 2.9 & 3 & $---^{a}$ \\
\hline 9 & 69.1 & 75 & 4.4 & 3 & 3 \\
\hline 10 & 64.8 & 75 & 3.5 & 3 & 2 \\
\hline
\end{tabular}

a The single-operator standard deviation of the extraction procedure can not be determined.

Table 76. Estimated single-Operator Precision of the sequential Batch Extraction Method Using Acidic Extraction Fluid in Extracting Sodium from the Spray Dryer Waste, $\mu \mathrm{g} / \mathrm{g}$

\begin{tabular}{|c|c|c|c|c|c|}
\hline $\begin{array}{r}\text { con } \\
\text { in } \\
\text { Extract }\end{array}$ & $\begin{array}{l}\text { Mean } \\
\text { ncentration } \\
\text { in Extract } \\
\overline{\bar{x}}\end{array}$ & $\begin{array}{c}\text { Mean } \\
\text { Concentration } \\
\text { in Analytical } \\
\text { standard } \\
\overline{\mathrm{x}}_{\mathrm{a}}\end{array}$ & $\begin{array}{c}\text { Single- } \\
\text { Operator } \\
\text { Precision } \\
\text { s. }_{\circ}\end{array}$ & $\begin{array}{c}\text { single- } \\
\text { operator } \\
\text { Analytical } \\
\text { Precision } \\
\text { soa }\end{array}$ & $\begin{array}{c}\text { Estimated single- } \\
\text { Operator Precision } \\
\text { of the Extraction } \\
\text { Procedure } \\
\text { see }_{o}\end{array}$ \\
\hline 1 & 3,515 & 3,669 & 50 & 161 & $--^{a}$ \\
\hline 2 & 224 & 116 & 10 & 6 & 8 \\
\hline 3 & 100 & 116 & 5 & 6 & $\ldots-^{a}$ \\
\hline 4 & 96 & 116 & 6 & 6 & 0 \\
\hline 5 & 98 & 116 & 5 & 6 & $--^{\mathbf{a}}$ \\
\hline 6 & 62 & 48 & 3 & 3 & 0 \\
\hline 7 & 50 & 48 & 2 & 3 & $--^{a}$ \\
\hline 8 & 60 & 48 & 5 & 3 & 4 \\
\hline 9 & 60 & 48 & 4 & 3 & 3 \\
\hline 10 & 51 & 48 & 4 & 3 & 3 \\
\hline
\end{tabular}

a The single-operator standard deviation of the extraction procedure can not be determined. 
Table 77. Estimated single-operator Precision of the sequential Batch Extraction Method Using Acidic Extraction Fluid in Extracting Strontiun from the Spray Dryer Waste, $\mu \mathrm{g} / \mathrm{g}$

\begin{tabular}{|c|c|c|c|c|c|}
\hline $\begin{array}{r}\text { Cono } \\
\text { in } \\
\text { Extract }\end{array}$ & $\begin{array}{l}\text { Mean } \\
\text { sentration } \\
\text { Extract } \\
\bar{x}\end{array}$ & $\begin{array}{c}\text { Mean } \\
\text { Concentration } \\
\text { in Analytical } \\
\text { standard } \\
\overline{\bar{s}_{a}}\end{array}$ & $\begin{array}{c}\text { single- } \\
\text { operator } \\
\text { Precision } \\
\text { s. }\end{array}$ & $\begin{array}{l}\text { single- } \\
\text { operator } \\
\text { Analytical } \\
\text { Precision } \\
\text { soa }\end{array}$ & $\begin{array}{c}\text { Estimated single- } \\
\text { operator Precision } \\
\text { of the Extraction } \\
\text { Procedure } \\
\text { soe }\end{array}$ \\
\hline 1 & 214 & 188 & 6 & 3 & 5 \\
\hline 2 & 98 & 46.7 & 4 & 1 & 4 \\
\hline 3 & 61.9 & 46.7 & 4.1 & 1 & 4 \\
\hline 4 & 47.6 & 46.7 & 1.6 & 1 & 1 \\
\hline 5 & 42.7 & 46.7 & 1.5 & 1 & 1 \\
\hline 6 & 32.2 & 19 & 1.4 & 0.2 & 1.4 \\
\hline 7 & 26.8 & 19 & 1.2 & 0.2 & 1.2 \\
\hline 8 & 24.8 & 19 & 0.8 & 0.2 & 0.8 \\
\hline 9 & 25.4 & 19 & 3.3 & 0.2 & 3.3 \\
\hline 10 & 19 & 19 & 1 & 0.2 & 1 \\
\hline
\end{tabular}

Table 78. Estimated single-operator precision of the sequential Batch Extraction Method Using Acidic Extraction Fluid in Extracting Barium Irom the Composite Mining Waste, $\mu g / g$

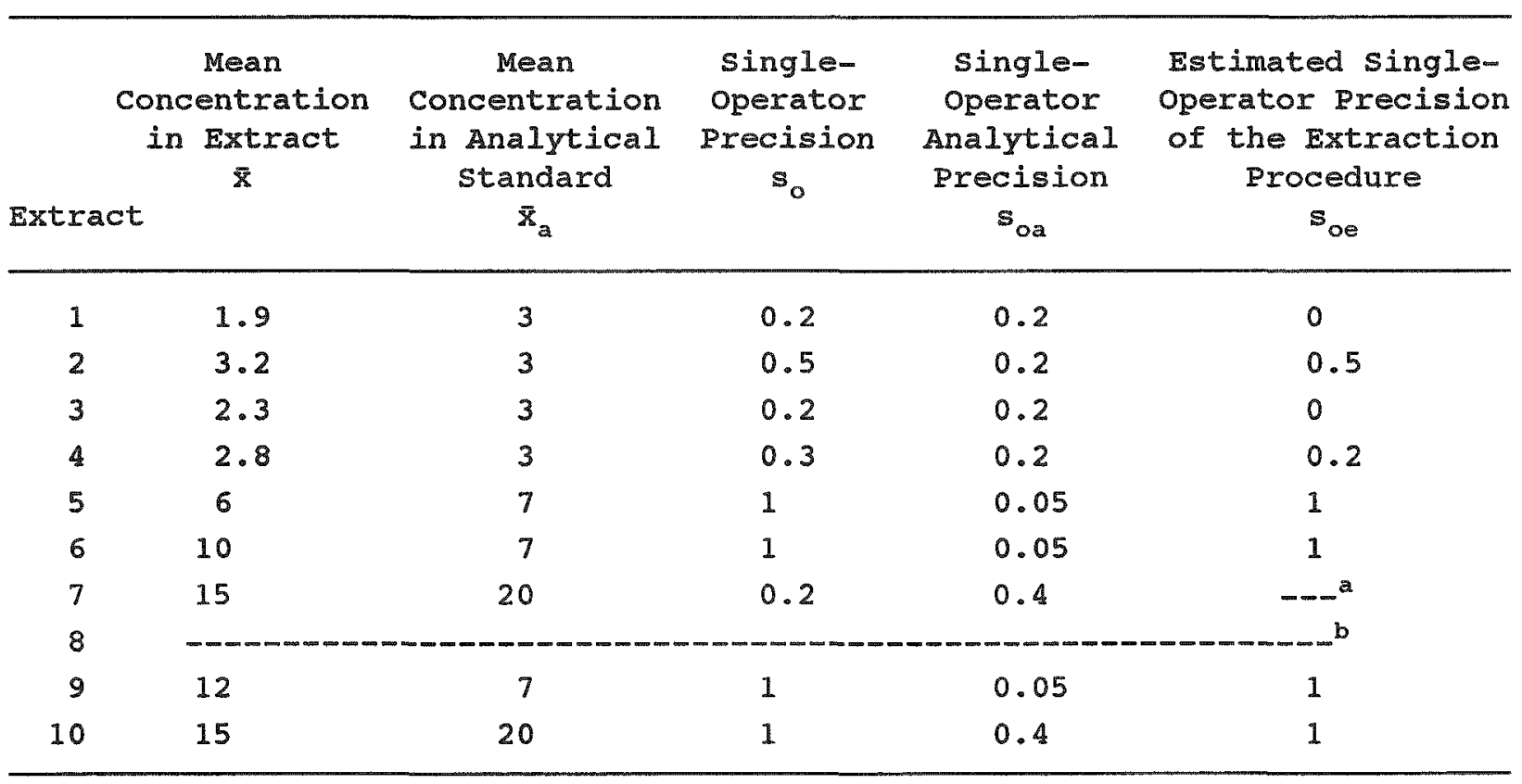

a The single-operator standard deviation of the extraction procedure can not be determined.

b calculations can not be performed because the data sets contain "Iess-than" values. 
Table 79. Estimated Single-operator Precision of the sequential Batch Extraction Method Using Acidic Extraction Fluid in Extracting Calcium from the Composite Mining Waste, $\mu \mathrm{g} / \mathrm{g}$

\begin{tabular}{|c|c|c|c|c|c|}
\hline $\begin{array}{r}\text { con } \\
\text { in } \\
\text { Extract }\end{array}$ & $\begin{array}{l}\text { Mean } \\
\text { centration } \\
\text { Extract } \\
\bar{x}\end{array}$ & $\begin{array}{c}\text { Mean } \\
\text { Concentration } \\
\text { in Analytical } \\
\text { Standard } \\
\overline{\mathrm{x}}_{\mathrm{a}}\end{array}$ & $\begin{array}{c}\text { single- } \\
\text { Operator } \\
\text { Precision } \\
\text { s. }_{\text {o }}\end{array}$ & $\begin{array}{c}\text { Single- } \\
\text { Operator } \\
\text { Analytical } \\
\text { Precision } \\
\text { soa }\end{array}$ & $\begin{array}{c}\text { Estimated single- } \\
\text { Operator Precision } \\
\text { of the Extraction } \\
\text { Procedure } \\
\text { soe }_{\text {of }}\end{array}$ \\
\hline 1 & 1,815 & 1,614 & 139 & 33 & 135 \\
\hline 2 & 628 & 622 & 27 & 9 & 25 \\
\hline 3 & 448 & 622 & 22 & 9 & 20 \\
\hline 4 & 383 & 248 & 17 & 5 & 16 \\
\hline 5 & 268 & 248 & 14 & 5 & 13 \\
\hline 6 & 229 & 248 & 11 & 5 & 10 \\
\hline 7 & 228 & 248 & 9 & 5 & 7 \\
\hline 8 & 230 & 248 & 19 & 5 & 18 \\
\hline 9 & 222 & 248 & 19 & 5 & 18 \\
\hline 10 & 214 & 248 & 12 & 5 & 11 \\
\hline
\end{tabular}

Table 80. Estimated single-operator Precision of the sequential Batch Extraction Method Using Acidic Extraction Fluid in Extracting Lead from the Composite Mining Waste, $\mu \mathrm{g} / \mathrm{g}$

\begin{tabular}{|c|c|c|c|c|c|}
\hline $\begin{array}{r}\text { con } \\
\text { ir } \\
\text { Extract }\end{array}$ & $\begin{array}{l}\text { Mean } \\
\text { oncentration } \\
\text { in Extract } \\
\overline{\bar{x}}\end{array}$ & $\begin{array}{c}\text { Mean } \\
\text { Concentration } \\
\text { in Analytical } \\
\text { Standard } \\
\overline{\mathrm{x}}_{\mathrm{a}}\end{array}$ & $\begin{array}{c}\text { single- } \\
\text { Operator } \\
\text { Precision } \\
\text { s. }_{0}\end{array}$ & $\begin{array}{c}\text { single- } \\
\text { Operator } \\
\text { Analytical } \\
\text { Precision } \\
\text { soa }\end{array}$ & $\begin{array}{c}\text { Estimated Single- } \\
\text { Operator Precision } \\
\text { of the Extraction } \\
\text { Procedure } \\
\end{array}$ \\
\hline 1 & 63 & 91 & 3 & 3 & 0 \\
\hline 2 & 73 & 91 & 6 & 3 & $5^{a}$ \\
\hline
\end{tabular}

a Values for the other extracts can not be calculated because the data sets contain "less-than" values. 
Table 81. Estimated single-operator Precision of the sequential Batch Extraction Method Using Acidic Extraction Fluid in Extracting Magnesium from the Composite Mining Waste, $\mu \mathrm{g} / \mathrm{g}$

\begin{tabular}{|c|c|c|c|c|c|}
\hline $\begin{array}{r}\text { con } \\
\text { in } \\
\text { Extract }\end{array}$ & $\begin{array}{l}\text { Mean } \\
\text { ncentration } \\
\text { in Extract } \\
\quad \bar{x}\end{array}$ & $\begin{array}{c}\text { Mean } \\
\text { Concentration } \\
\text { in Analytical } \\
\text { standard } \\
\overline{\mathrm{x}}_{\mathrm{a}}\end{array}$ & $\begin{array}{c}\text { Single- } \\
\text { operator } \\
\text { Precision } \\
\mathbf{s}_{\circ}\end{array}$ & $\begin{array}{c}\text { single- } \\
\text { Operator } \\
\text { Analytical } \\
\text { Precision } \\
\text { soa }\end{array}$ & $\begin{array}{c}\text { Estimated single- } \\
\text { operator Precision } \\
\text { of the Extraction } \\
\text { Procedure } \\
\text { soe }^{\text {op }}\end{array}$ \\
\hline 1 & 107 & 102 & 7 & 1 & 7 \\
\hline 2 & 20 & 14 & 1 & 0.4 & 1 \\
\hline 3 & 12 & 14 & 1 & 0.4 & 1 \\
\hline 4 & 11 & 14 & 1 & 0.4 & 1 \\
\hline 5 & 10 & 14 & 1 & 0.4 & 1 \\
\hline 6 & 6 & 6 & 0.4 & 0.2 & 0.3 \\
\hline 7 & 5 & 6 & 0.3 & 0.2 & 0.2 \\
\hline 8 & 5 & 6 & 1 & 0.2 & 1 \\
\hline 9 & 6 & 6 & 1 & 0.2 & 1 \\
\hline 10 & 5 & 6 & 0.3 & 0.2 & 0.2 \\
\hline
\end{tabular}

Table 82. Estimated single-operator Precision of the sequential Batch Extraction Method Using Acidic Extraction Fluid in Extracting Manganese from the Composite Mining Waste, $\mu \mathrm{g} / \mathrm{g}$

\begin{tabular}{|c|c|c|c|c|c|}
\hline con & $\begin{array}{l}\text { Mean } \\
\text { ncentration } \\
\text { Ln Extract } \\
\overline{\bar{x}}_{\overline{\mathrm{x}}}\end{array}$ & $\begin{array}{c}\text { Mean } \\
\text { Concentration } \\
\text { in Analytical } \\
\text { Standard } \\
\bar{x}_{\mathrm{a}}\end{array}$ & $\begin{array}{l}\text { Single- } \\
\text { operator } \\
\text { Precision } \\
\text { s. }\end{array}$ & $\begin{array}{c}\text { Single- } \\
\text { Operator } \\
\text { Analytical } \\
\text { Precision } \\
\text { soa }\end{array}$ & $\begin{array}{c}\text { Estimated Single- } \\
\text { Operator Precision } \\
\text { of the Extraction } \\
\text { Procedure } \\
\text { soe }\end{array}$ \\
\hline 1 & 36.7 & 39.9 & 1.1 & 0.5 & 1.0 \\
\hline 2 & 9 & 6.1 & 1 & 0.2 & 1 \\
\hline 3 & 3.6 & 6.1 & 0.3 & 0.2 & 0.2 \\
\hline 4 & 1.8 & 1.0 & 0.2 & 0.03 & 0.2 \\
\hline 5 & 0.65 & 1.0 & 0.12 & 0.03 & $0.1^{\mathrm{a}}$ \\
\hline
\end{tabular}

a Values for the other extracts can not be calculated because the data sets contain "less-than" values. 
Table 83. Estimated single-operator Precision of the sequential Batch Extraction Method Using Acidic Extraction Fluid in Extracting silicon from the composite Mining Waste, $\mu \mathrm{g} / \mathrm{g}$

\begin{tabular}{|c|c|c|c|c|c|}
\hline $\begin{array}{r}\text { Conc } \\
\text { in } \\
\text { Extract }\end{array}$ & $\begin{array}{l}\text { Mean } \\
\text { entration } \\
\text { Extract } \\
\overline{\mathrm{z}}\end{array}$ & $\begin{array}{c}\text { Mean } \\
\text { Concentration } \\
\text { in Analytical } \\
\text { standard } \\
\bar{x}_{\mathrm{a}}\end{array}$ & $\begin{array}{c}\text { single- } \\
\text { operator } \\
\text { Precision } \\
\text { s。 }\end{array}$ & $\begin{array}{c}\text { single- } \\
\text { operator } \\
\text { Analytical } \\
\text { Precision } \\
\text { soa }_{\text {op }}\end{array}$ & $\begin{array}{c}\text { Estimated single- } \\
\text { Operator Precision } \\
\text { of the Extraction } \\
\text { Procedure } \\
\text { soe }_{\text {o }}\end{array}$ \\
\hline 1 & 55 & 44 & 2 & 1 & 2 \\
\hline 2 & 51 & 44 & 3 & 1 & 3 \\
\hline 3 & 35 & 34 & 3 & 1 & 3 \\
\hline 4 & 26 & 34 & 2 & 1 & 2 \\
\hline 5 & 20 & 13 & 2 & 1 & 2 \\
\hline 6 & 15 & 13 & 1 & 1 & 0 \\
\hline 7 & 14 & 13 & 1 & 1 & 0 \\
\hline 8 & 14 & 13 & 1 & 1 & 0 \\
\hline 9 & 14 & 13 & 1 & 1 & 0 \\
\hline 10 & 14 & 13 & 1 & 1 & 0 \\
\hline
\end{tabular}

Table 84. Estimated single-Operator Precision of the sequential Batch Extraction Method Using Acidic Extraction Fluid in Extracting zinc from the Composite Mining Waste, $\mu \mathrm{g} / \mathrm{g}$

\begin{tabular}{|c|c|c|c|c|c|}
\hline $\begin{array}{r}\text { con } \\
\text { in } \\
\text { Extract }\end{array}$ & $\begin{array}{l}\text { Mean } \\
\text { ncentration } \\
\text { in Extract } \\
\overline{\bar{x}}\end{array}$ & $\begin{array}{c}\text { Mean } \\
\text { Concentration } \\
\text { in Analytical } \\
\text { standard } \\
\bar{x}\end{array}$ & $\begin{array}{c}\text { single- } \\
\text { operator } \\
\text { Precision } \\
\text { s. }\end{array}$ & $\begin{array}{c}\text { Single- } \\
\text { Operator } \\
\text { Analytical } \\
\text { Precision } \\
\text { s. }\end{array}$ & $\begin{array}{c}\text { Estimated Single- } \\
\text { Operator Precision } \\
\text { of the Extraction } \\
\text { Procedure } \\
\text { s }\end{array}$ \\
\hline 1 & 297 & 312 & 31 & 6 & 30 \\
\hline 2 & 39 & 41.6 & 2 & 0.8 & 2 \\
\hline 3 & 13 & 3.6 & 1 & 1.0 & $0^{a}$ \\
\hline
\end{tabular}

a Values for the other extracts can not be calculated because the data sets contain "less-than" values. 
In this study, it was not economically practical to determine analytical precision using actual extracts of the wastes because of the extensive number of analyses that would have been required. The analytical standards were, therefore, used to determine analytical precision. Calculation of the standard deviation of the extraction procedure can provide only an approximation because the analytical standards do not contain the specific matrix resulting from the interaction of the extraction fluid and solid waste. In addition, the analytical standards are limited to three concentration levels for each element. For some of the extracts, the elemental concentration in the extract varies significantly from the element's closest concentration in the analytical standards. However, for the calculations performed in this study, $70 \%$ of the elemental concentrations in the extracts vary by less than 258 from their concentrations in the analytical standard used to determine analytical precision.

Because of the way in which the total precision and analytical precision were determined, in some cases, the analytical standard deviation values, $\mathbf{s}_{\mathbf{a}}$ and $s_{o a}$ are larger than the total standard deviation values, $s$ and $s_{0}$. In these cases, the precision of the extraction procedure can not be determined. A discussion of the estimated multiple-laboratory and single-operator precision of the extraction procedure for the elements of interest in the spray dryer waste and the composite mining waste is given below.

Extraction of Aluminum from the spray Dryer Waste. The data used for estimating the precision of the sequential batch extraction method in extracting aluminum from the spray dryer waste are given in Tables 55 (multiple-laboratory) and 70 (single-operator). The estimated singleoperator precision of the extraction procedure can be determined for extracts one through five only. Figure 1 is a plot of the estimated percent relative standard deviation of the extraction method versus mean concentration of aluminum in the spray dryer waste extract. The estimated standard deviation of the extraction procedure varies with the aluminum concentration extracted from the spray dryer waste over the concentration range of 25.5 to $183 \mu \mathrm{g} / \mathrm{g}$. over this concentration range, the estimated multiple-laboratory precision of the extraction procedure for extracting aluminum from the spray dryer waste averages approximately $5 \%$, and the estimated single-operator precision ranges from 0 to $4 \%$.

Extraction of Barium from the spray Dryer waste. The data used for estimating the precision of the sequential batch extraction method in extracting barium from the spray dryer waste are given in Tables 56 (multiple-laboratory) and 71 (single-operator). The estimated multiplelaboratory precision of the extraction procedure can be determined for only extracts one through five. Figure 2 is a plot of the estimated percent relative standard deviation of the extraction method versus mean concentration of barium in the spray dryer waste extract. The estimated multiple-laboratory standard deviation of the extraction procedure for extracting barium from the spray dryer waste varies with the barium concentration for the range of 14 to $62 \mu \mathrm{g} / \mathrm{g}$. The estimated multiplelaboratory percent relative standard deviation for this concentration range is high, ranging from 10 to 55\%, while the estimated single-operator percent relative standard deviation is much lower, averaging about $4 \%$. 


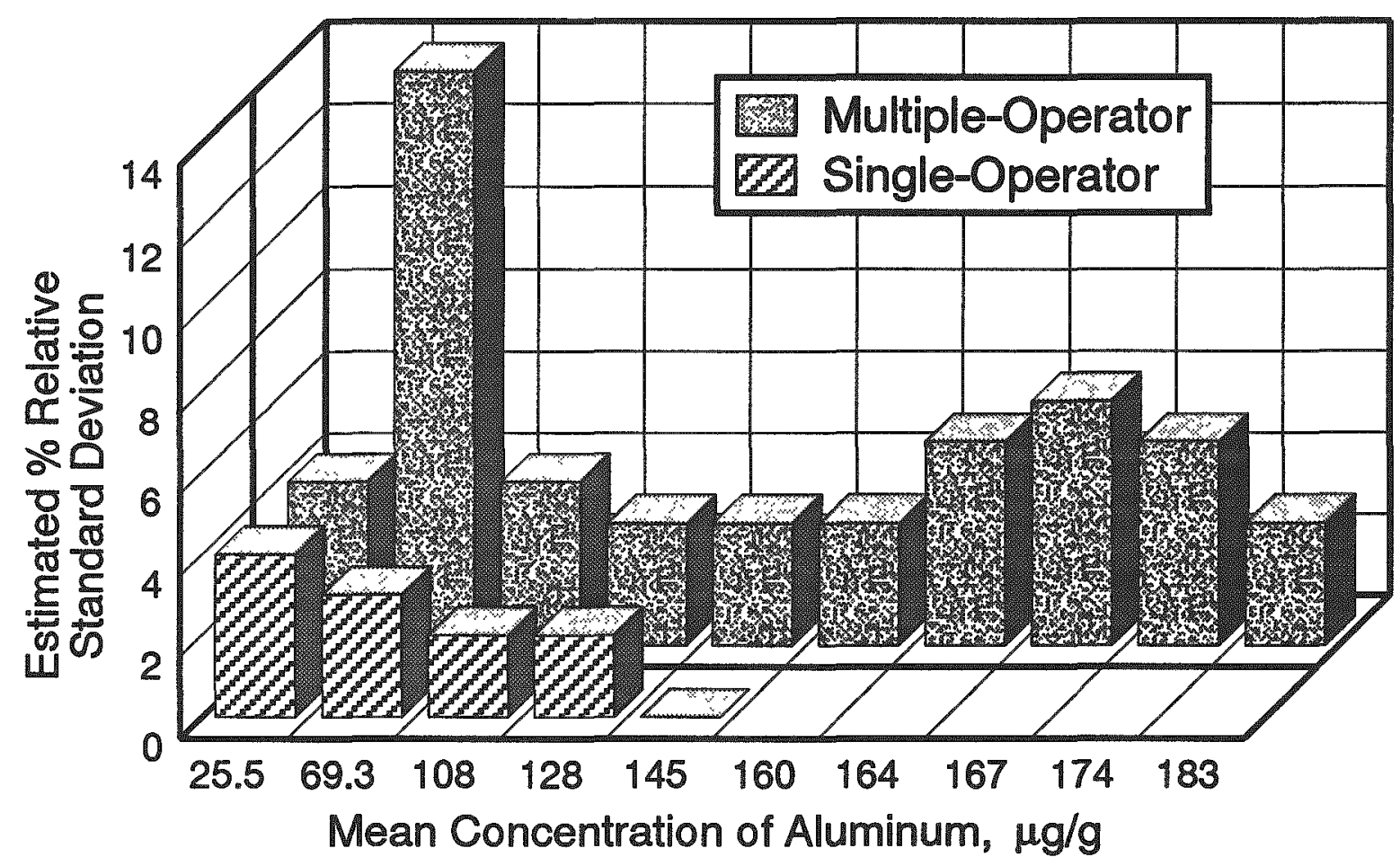

Figure 1. Estimated Precision of the Sequential Batch Extraction Method for Aluminum in the Spray Dryer Waste

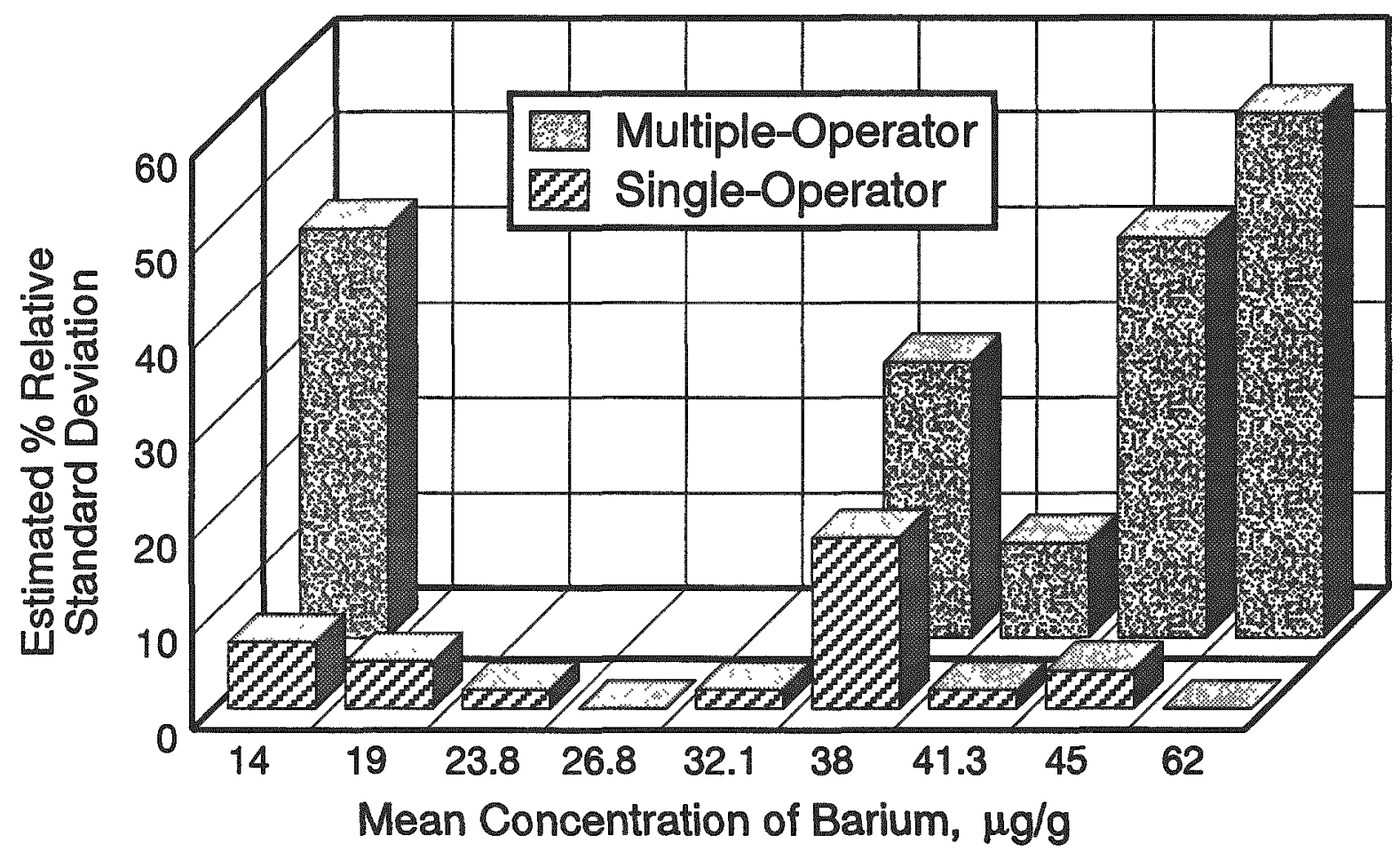

Figure 2. Estimated Precision of the Sequential Batch Extraction Method for Barium in the Spray Dryer Waste 
Extraction of Boron from the spray Dryer Waste. The multiple-laboratory precision of the sequential batch extraction method for extracting boron can not be estimated for the spray dryer waste extracts because the analytical standard deviation values (Table 57) are larger than the total standard deviation values. As previously mentioned, this is due to the way in which the total precision and analytical precision were determined. The data used for estimating the single-operator precision of the sequential batch extraction method in extracting boron from the spray dryer waste are given in Table 72. Figure 3 is a plot of the estimated single-operator percent relative standard deviation of the extraction method versus mean concentration of boron in the spray dryer waste extract. The estimated single-operator precision of the extraction procedure for extracts one through five ranges from 11 to 29 \% over a concentration range of approximately 2 to $3 \mu \mathrm{g} / \mathrm{g}$. For extracts six through ten, the estimated single-operator precision of the extraction procedure ranges from 6 to 8\% over a concentration range of 3.6 to $5.3 \mu \mathrm{g} / \mathrm{g}$. Figure 3 shows that for extracts six through ten, the relationship between the estimated singleoperator standard deviation and concentration is almost directly proportional.

Extraction of Calcium from the spray Dryer waste. The multiplelaboratory precision of the sequential batch extraction method for extracting calcium from the spray dryer waste can be estimated for only three of the spray dryer waste extracts (Table 58). The same is also true for singleoperator precision of the method (Table 73). The estimated percent relative standard deviation of the extraction method versus mean concentration of calcium in the spray dryer waste extract is given in Figure 4. At extract concentrations of approximately $3,800 \mu \mathrm{g} / \mathrm{g}$ and $3,100 \mu \mathrm{g} / \mathrm{g}$, the multiplelaboratory percent relative standard deviation of the method for extracting calcium from the spray dryer waste is 5 and $6 \%$. At an extract concentration of $1,831 \mu \mathrm{g} / \mathrm{g}$, it is much higher, at 20\%. The single-operator percent relative standard deviation values at these concentrations are much lower. The limited data make any discussion concerning the relationship between the calcium concentration in the extract and the precision of the method difficult.

Extraction of Chromium from the spray Dryer Waste. Multiple-laboratory precision and single-operator precision of the sequential batch extraction method for extracting chromiun from the spray dryer waste can be estimated for only the first two extracts (Tables 59 and 74). This is because the chromium data resulting from analysis of extracts three through ten by the collaborative study participants contain "less-than" values, and the data can not be statistically evaluated. As mentioned previously, an initial screening was performed to distinguish elements having concentrations below instrumental detection limits in the extracts. However, because some of the laboratories have different detection limits, "less-than" values for chromium were reported. The estimated percent relative standard deviation of the extraction method versus mean concentration of chromium in the spray dryer waste extract is given in Figure 5. At a chromium concentration in the spray dryer waste extract of $2.8 \mu \mathrm{g} / \mathrm{g}$, both the multiple-laboratory and singleoperator precision are estimated to be $7 \%$; and at a chromium concentration of $1.1 \mu \mathrm{g} / \mathrm{g}$, the multiple-laboratory precision of the extraction procedure is estimated to be $27 \%$, and the single-operator precision is estimated to be slightly better at $18 \%$. 


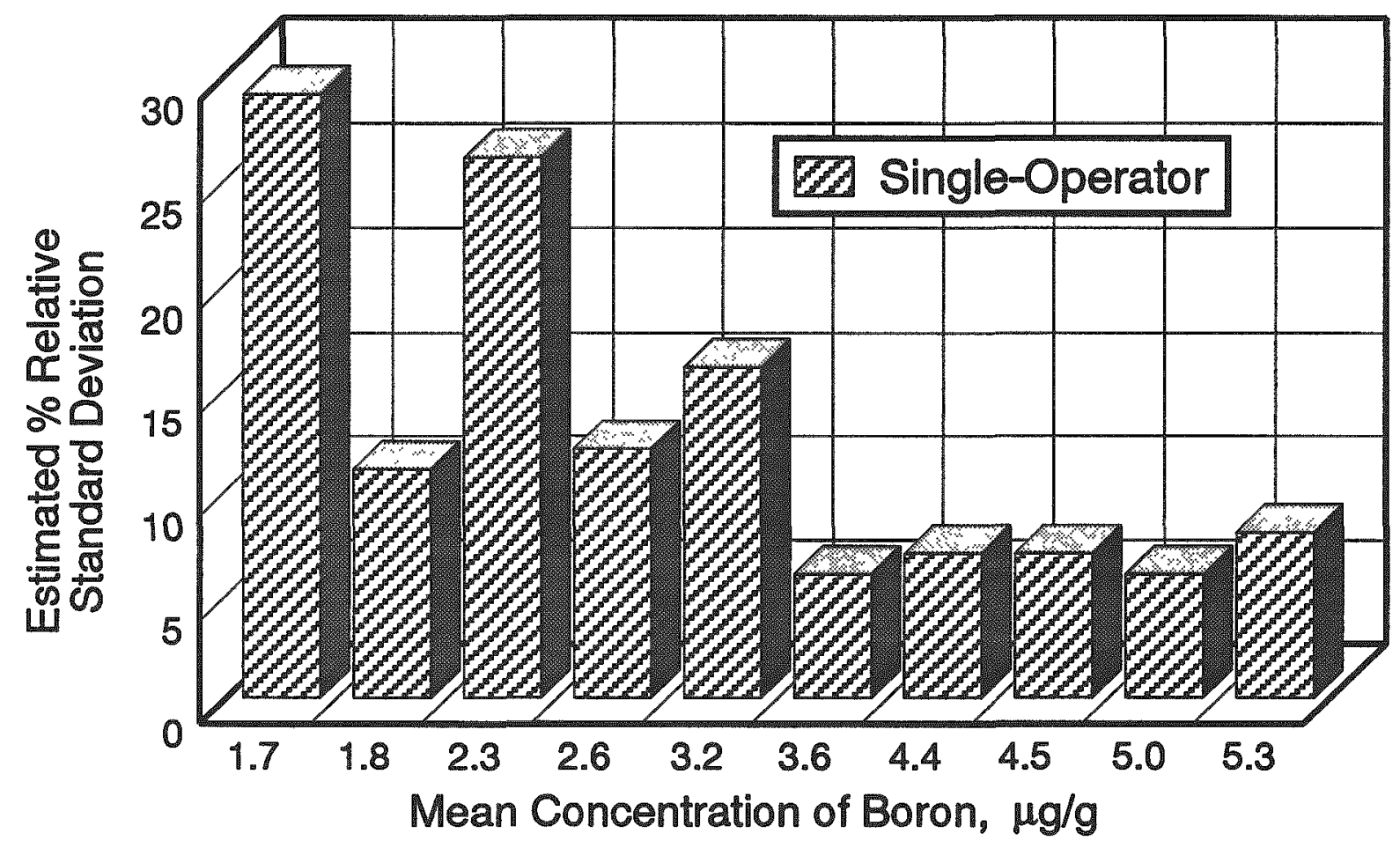

Figure 3. Estimated Precision of the Sequential Batch Extraction Method for Boron in the Spray Dryer Waste

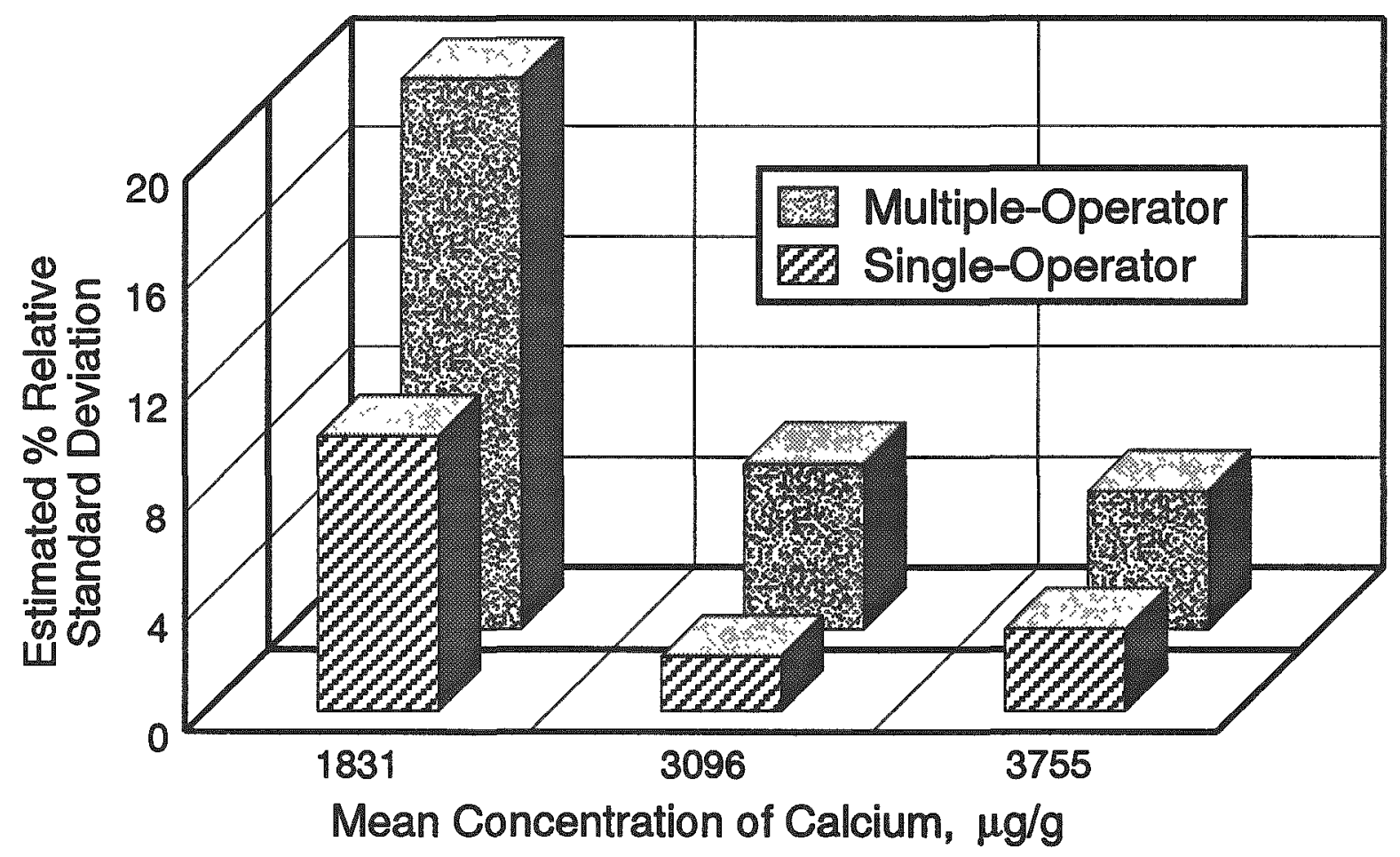

Figure 4. Estimated Precision of the Sequential Batch Extraction Method for Calcium in the Spray Dryer Waste 


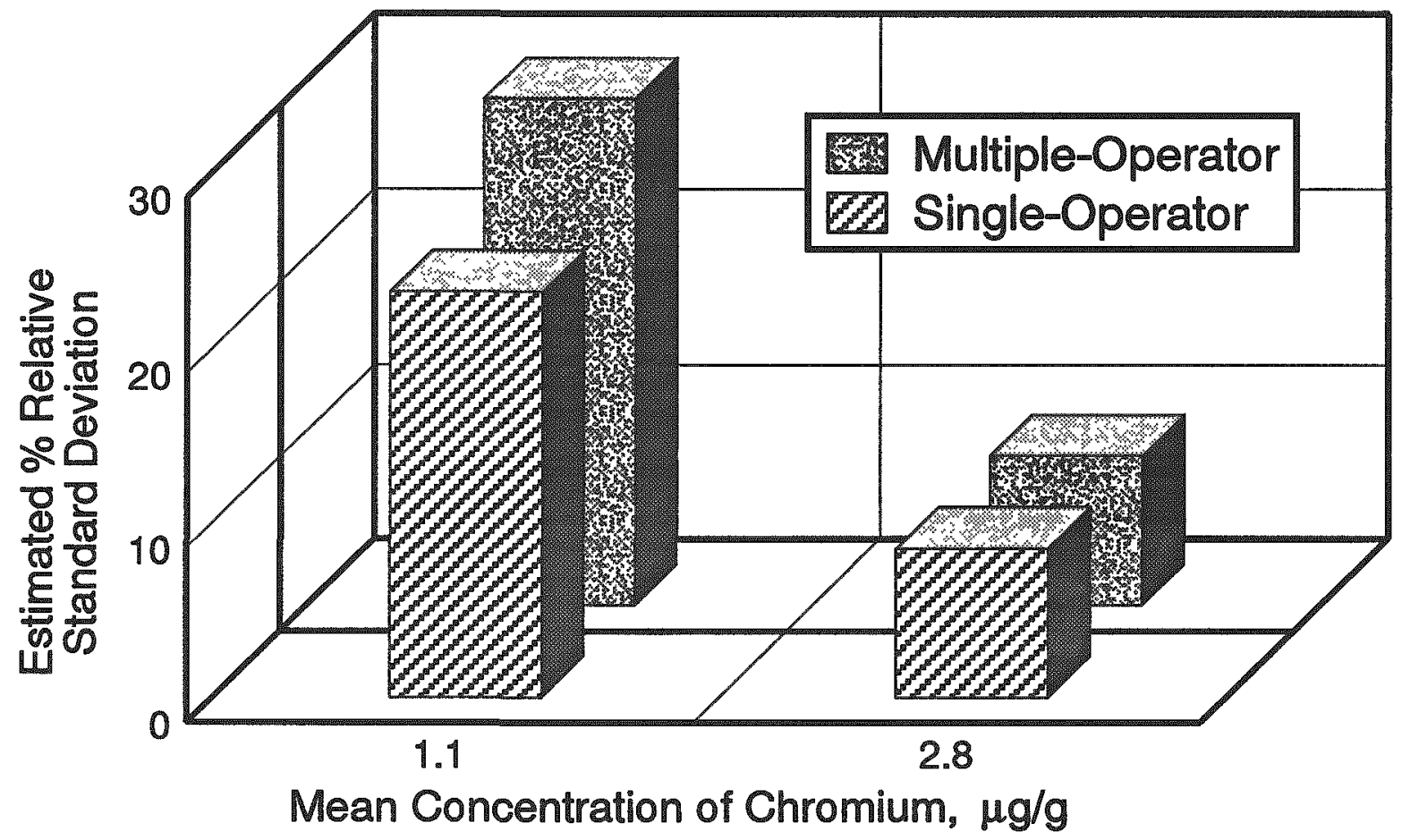

Figure 5. Estimated Precision of the Sequential Batch Extraction Method for Chromium in the Spray Dryer Waste

Extraction of silicon from the spray Dryer waste. The data used for estimating the precision of the sequential batch extraction method in extracting silicon from the spray dryer waste are given in Tables 60 (multiple-laboratory) and 75 (single-operator). Figure 6 is a plot of the estimated percent relative standard deviation of the extraction method versus mean concentration of silicon in the spray dryer waste extract. The estimated multiple-laboratory standard deviation of the extraction procedure varies with the concentration of silicon extracted over the concentration range of 69 to $181 \mu \mathrm{g} / \mathrm{g}$. The estimated multiple-laboratory percent relative standard deviation for this concentration range is 0 to $12 \%$, and the estimated single-operator percent relative standard deviation for the concentration range of 65 to $136 \mu \mathrm{g} / \mathrm{g}$ is 0 to 48 .

Extraction of Sodium from the spray Dryer waste. The multiple-laboratory precision of the sequential batch extraction method for extracting sodium from the spray dryer waste can be estimated for only three of the spray dryer waste extracts (Table 61), while the single-operator precision can be estimated for six extracts (Table 76). The estimated percent relative standard deviation of the extraction method versus mean concentration of sodium in the spray dryer waste extract is given in Figure 7 . The limited data for the multiple-laboratory standard deviation of the extraction procedure vary directly with the sodium concentration in the extract. The estimated multiple-laboratory precision for the extraction procedure over the concentration range covered by the three extracts is 17 to $28 \%$, and the estimated single-operator precision over the concentration range of 51 to 224 $\mu \mathrm{g} / \mathrm{g}$ is 0 to 78 . The estimated single-operator standard deviation also varies with concentration. 


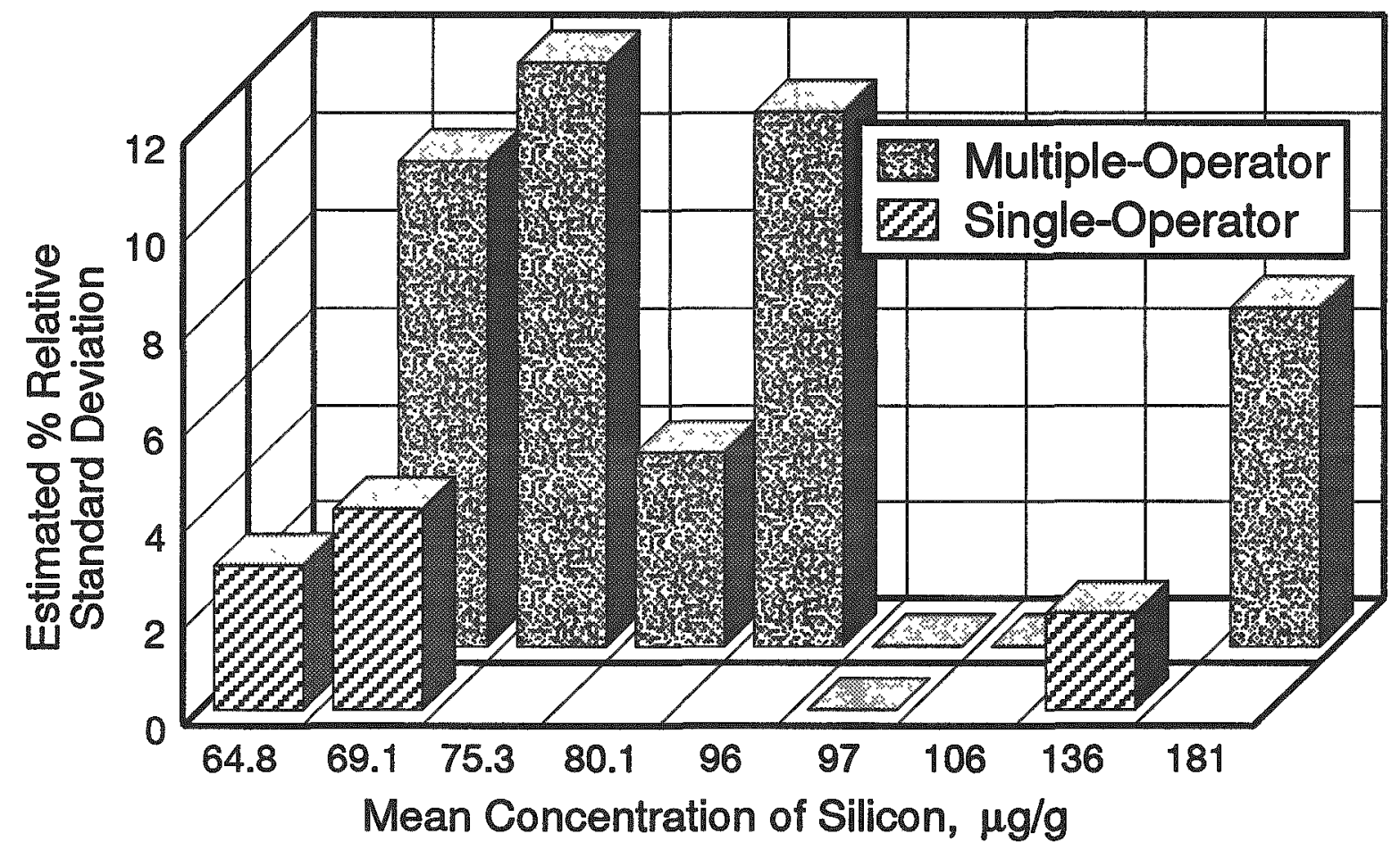

Figure 6. Estimated Precision of the Sequential Batch Extraction Method for Silicon in the Spray Dryer Waste

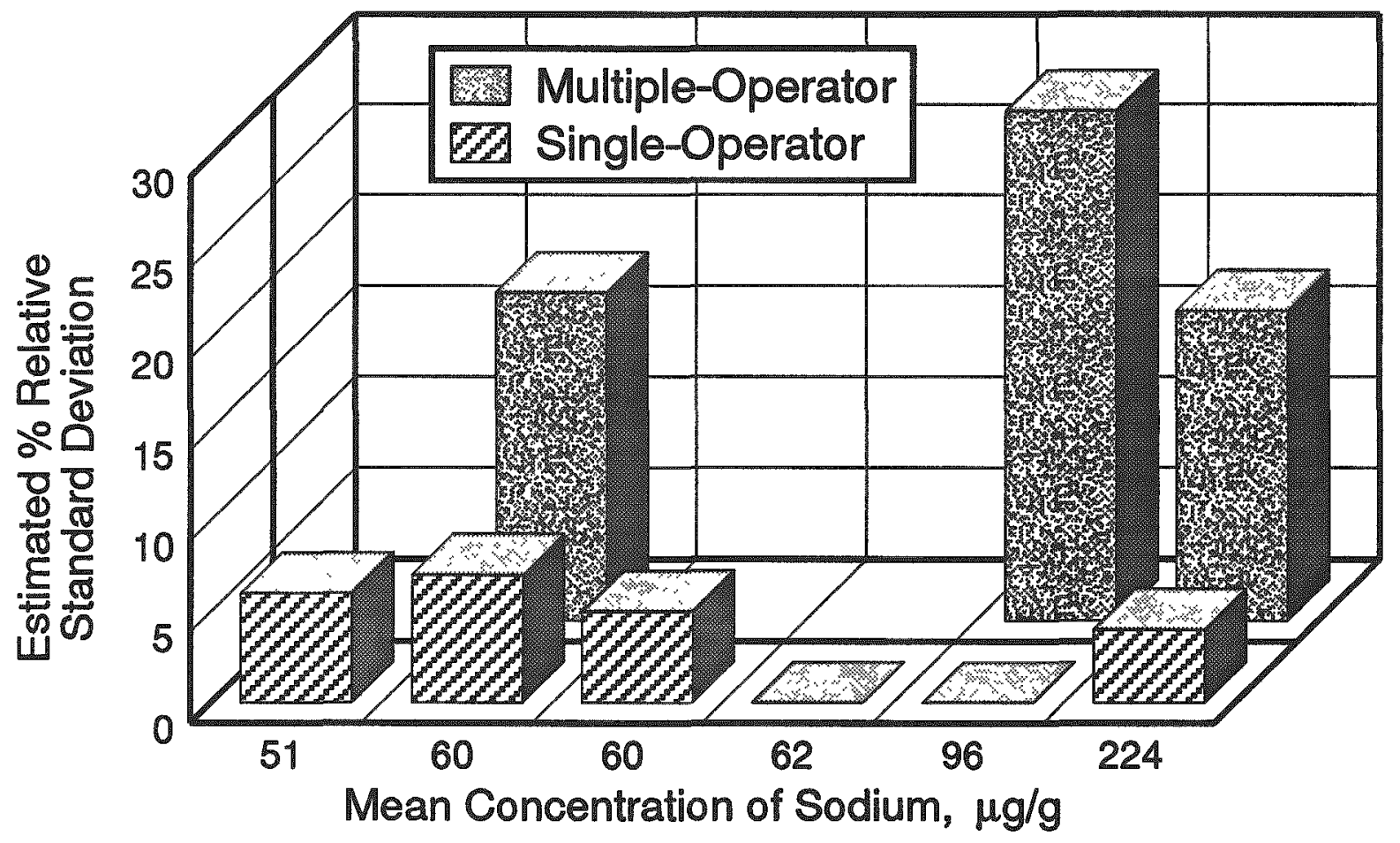

Figure 7. Estimated Precision of the Sequential Batch Extraction Method for Sodium in the Spray Dryer Waste 
Extraction of strontium from the Spray Dryer Waste. The data used for estimating the precision of the sequential batch extraction method in extracting strontium from the spray dryer waste are given in Tables 62 (multiple-laboratory) and 77 (single-operator). Figure 8 is a plot of the estimated percent relative standard deviation of the extraction method versus mean concentration of strontium in the spray dryer waste extract. The estimated multiple-laboratory precision for the extraction procedure averages about $7 \%$, while the estimated single-operator percent relative standard deviation averages about 5\%. Both the estimated multiple-laboratory and single-operator standard deviations of the extraction procedure for strontium appear to vary directly with concentration.

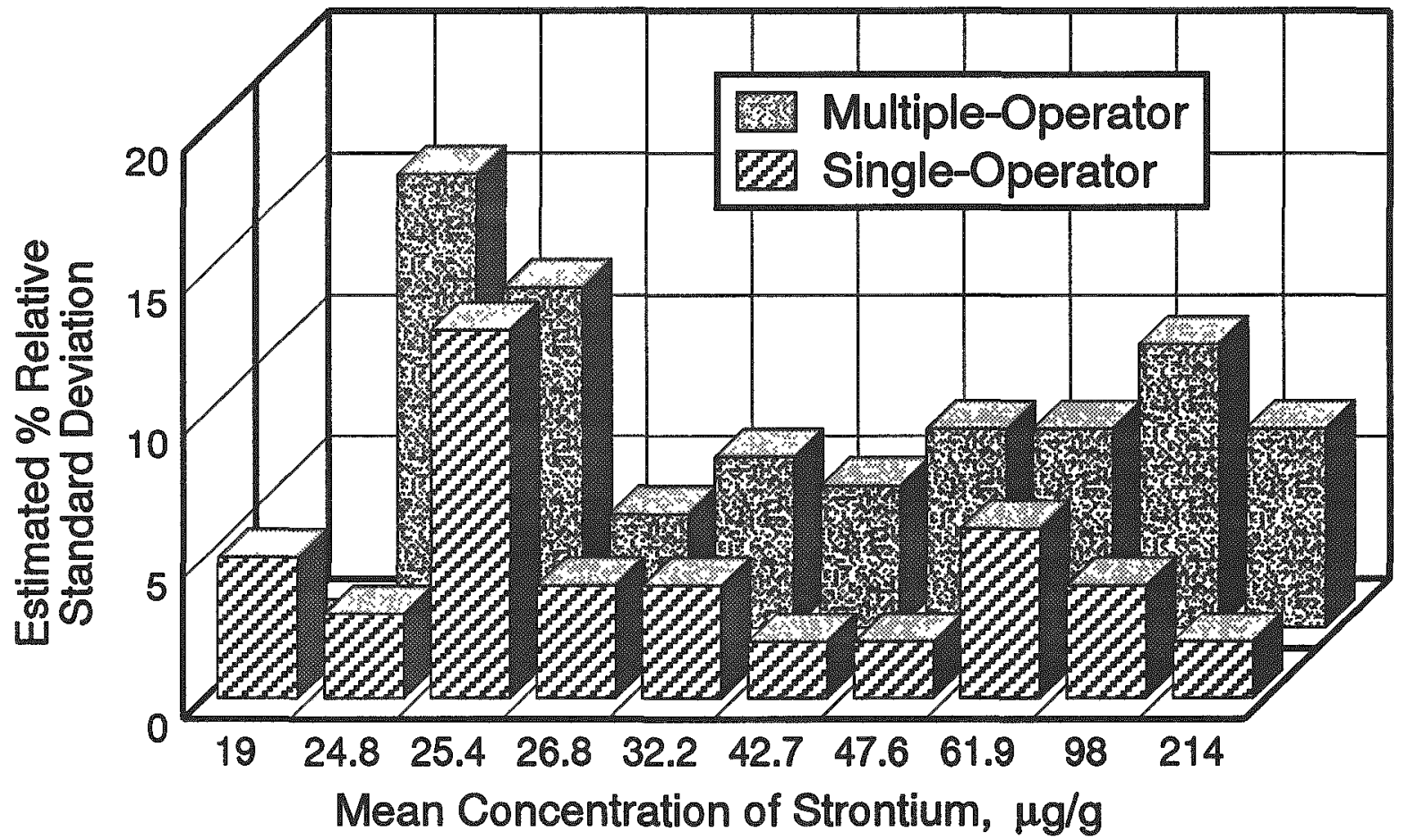

Figure 8. Estimated Precision of the Sequential Batch Extraction Method for Strontium in the Spray Dryer Waste

Extraction of Barium from the composite Mining waste. The multiplelaboratory precision of the sequential batch extraction method for extracting barium from the composite mining waste can not be estimated because the analytical standard deviation values are larger than the total standard deviation values (Table 63). As previously mentioned, this is due to the way in which the total precision and analytical precision were determined. The data used for estimating the single-operator precision of the seguential batch extraction method in extracting barium from the composite mining waste are given in Table 78. Figure 9 is a plot of the estimated single-operator percent relative standard deviation of the extraction method versus mean concentration of barium in the composite mining waste extract. For the concentrations of barium in the composite mining waste extracts, the estimated single-operator precision of the extraction procedure ranges from 0 to $17 \%$. Over the concentration range of 1.9 to $15 \mu \mathrm{g} / \mathrm{g}$, the estimated single-operator standard deviation ranges from 0 to $1 \mu \mathrm{g} / \mathrm{g}$. 


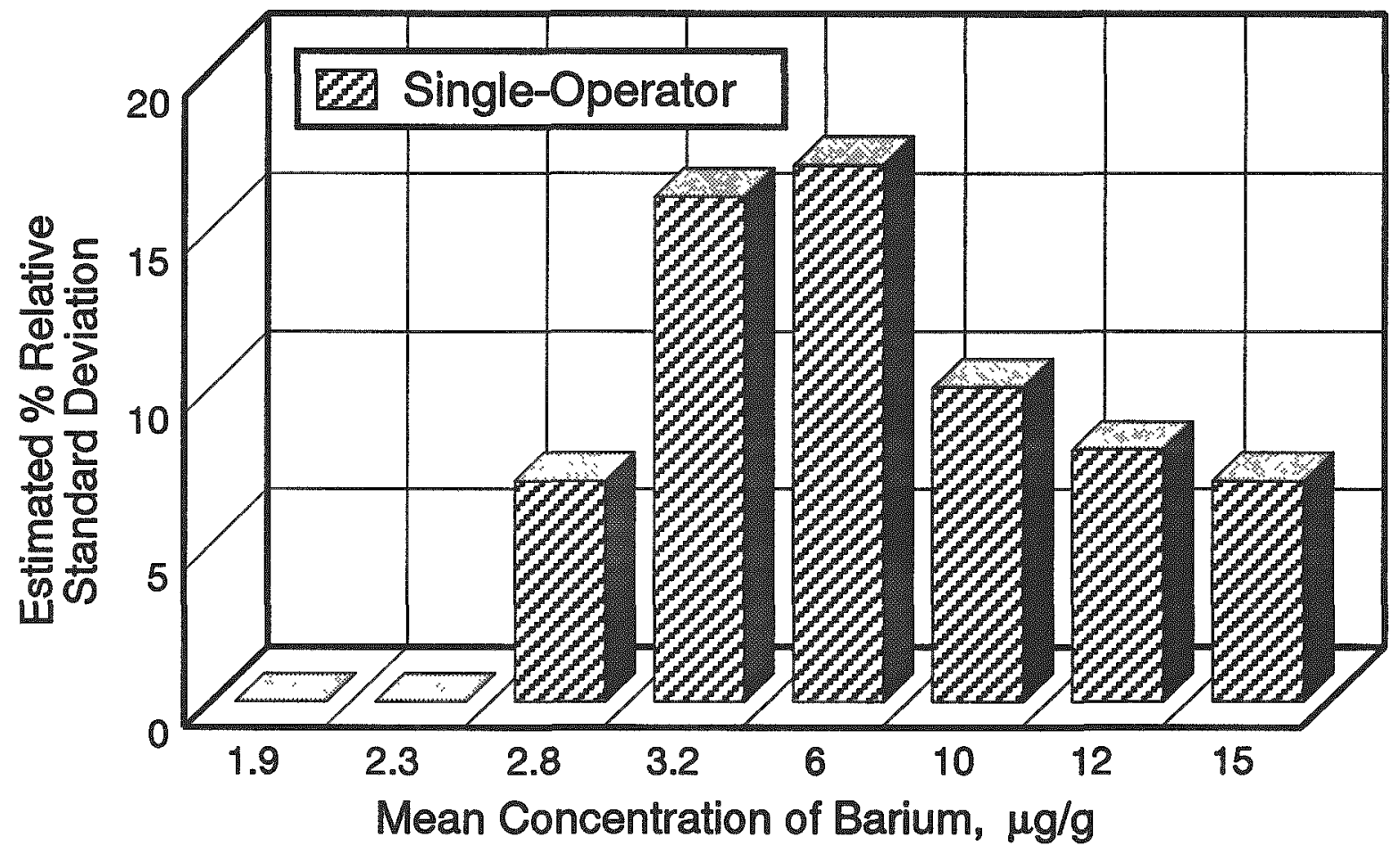

Figure 9. Estimated Precision of the Sequential Batch Extraction Method for Barium in the Composite Mining Waste

Extraction of Calcium from the composite Mining Waste. The data used for estimating the precision of the sequential batch extraction method in extracting calcium from the composite mining waste are given in Tables 64 (multiple-laboratory) and 79 (single-operator). Figure 10 is a plot of the estimated percent relative standard deviation of the extraction method versus mean concentration of calcium in the composite mining waste extract. The estimated standard deviation of the extraction procedure varies with the concentration of calcium extracted over the concentration range of 214 to $1,815 \mu \mathrm{g} / \mathrm{g}$. The estimated multiple-laboratory precision for the extraction procedure averages about $13 \%$, while the estimated single-operator percent relative standard deviation averages about $5 \%$.

Extraction of Lead from the Composite Mining Waste. Multiple-laboratory precision and single-operator precision of the sequential batch extraction method for extracting lead from the composite mining waste can be estimated for only the first two extracts (Tables 65 and 80 ). This is because the lead data resulting from analysis of extracts three through ten by the collaborative study participants contain "less-than" values, and the data can not be statistically evaluated. Figure 11 is a plot of the estimated percent relative standard deviation of the extraction method versus mean concentration of lead in the composite mining waste extract. At an extract concentration of $63 \mu \mathrm{g}$ lead/g composite mining waste, the multiple-laboratory precision of the extraction procedure is estimated to be $11 \%$, and the singleoperator precision is estimated to be 08 . At an extract concentration of 73 $\mu g$ lead/g composite mining waste, the multiple-laboratory precision of the extraction procedure is estimated to be $18 \%$, and the single-operator precision is estimated to be $7 \%$. 


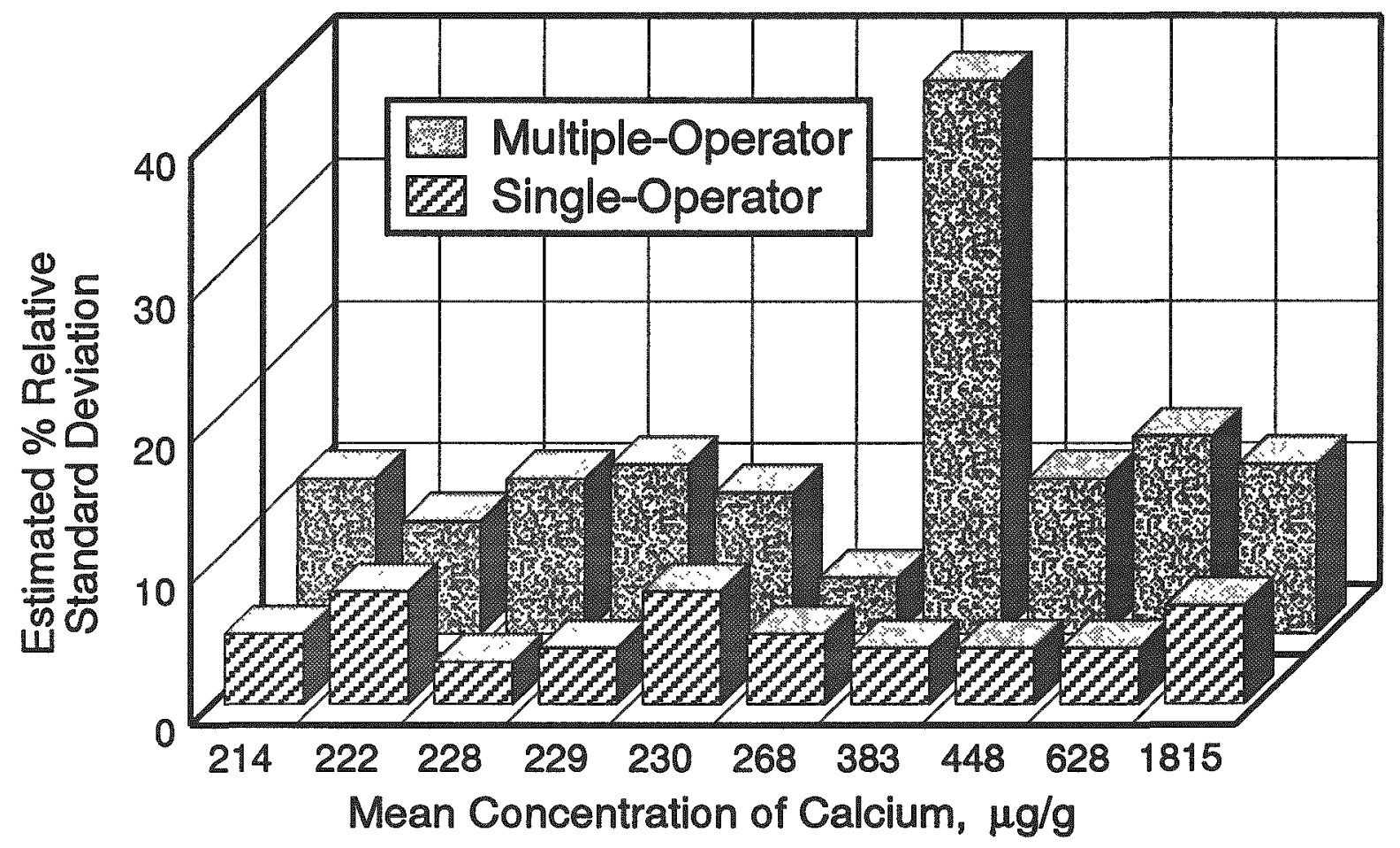

Figure 10. Estimated Precision of the Sequential Batch Extraction Method for Calcium in the Composite Mining Waste

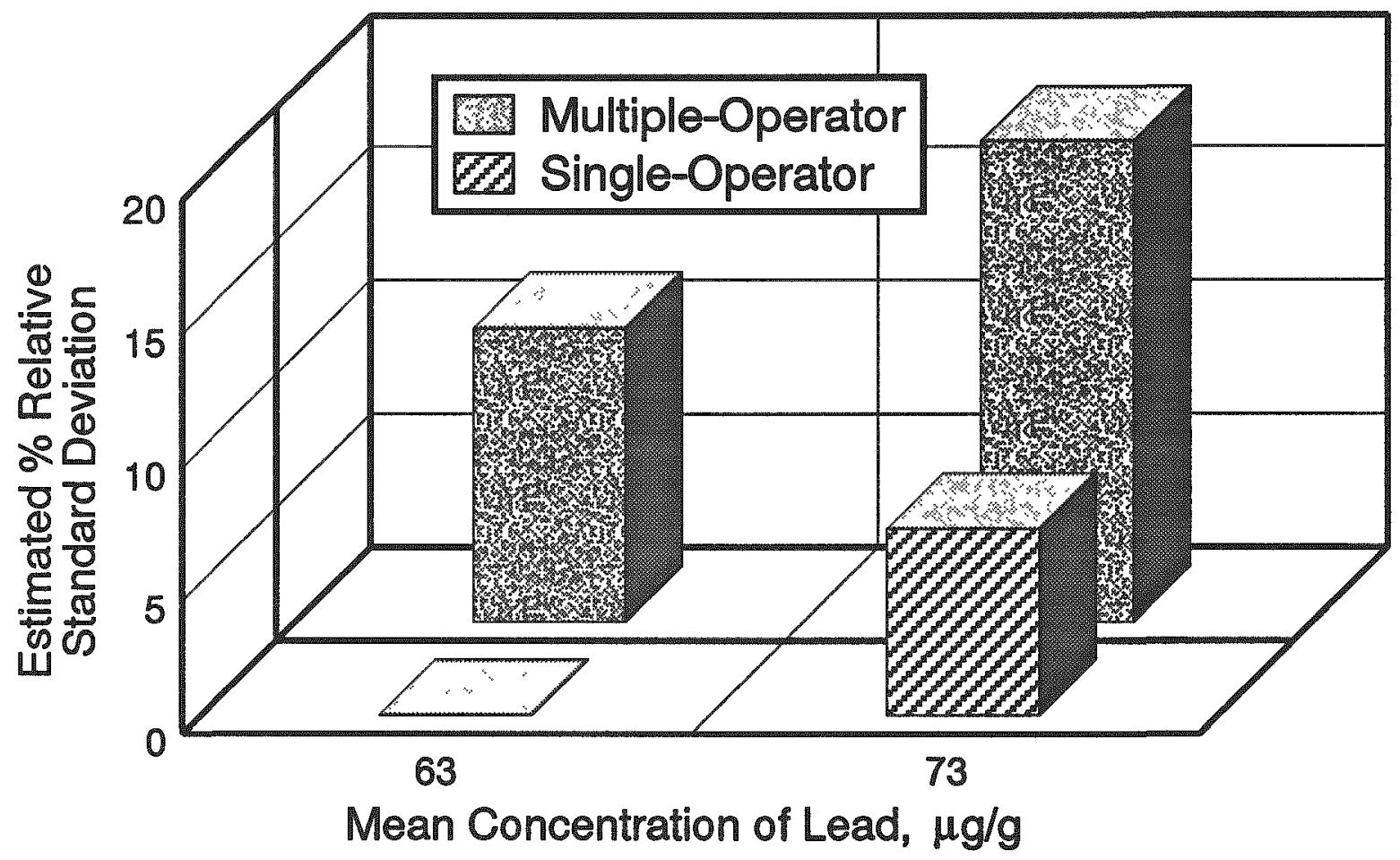

Figure 11. Estimated Precision of the Sequential Batch Extraction Method for Lead in the Composite Mining Waste 
Extraction of Magnesium from the Composite Mining Waste. The data used for estimating the precision of the sequential batch extraction method in extracting magnesium from the composite mining waste are given in Tables 66 (multiple-laboratory) and 81 (single-operator). Figure 12 is a plot of the estimated percent relative standard deviation of the extraction method versus mean concentration of magnesium in the composite mining waste extract. The estimated multiple-laboratory percent relative standard deviation for extraction of magnesium from the composite mining waste is high, averaging about $20 \%$ for the concentrations determined in the extracts. The estimated single-operator precision of the extraction procedure for magnesium is better, averaging about $9 \%$ over the concentration range. The estimated standard deviation of the extraction procedure for magnesium appears to vary directly with the concentration of magnesium extracted.

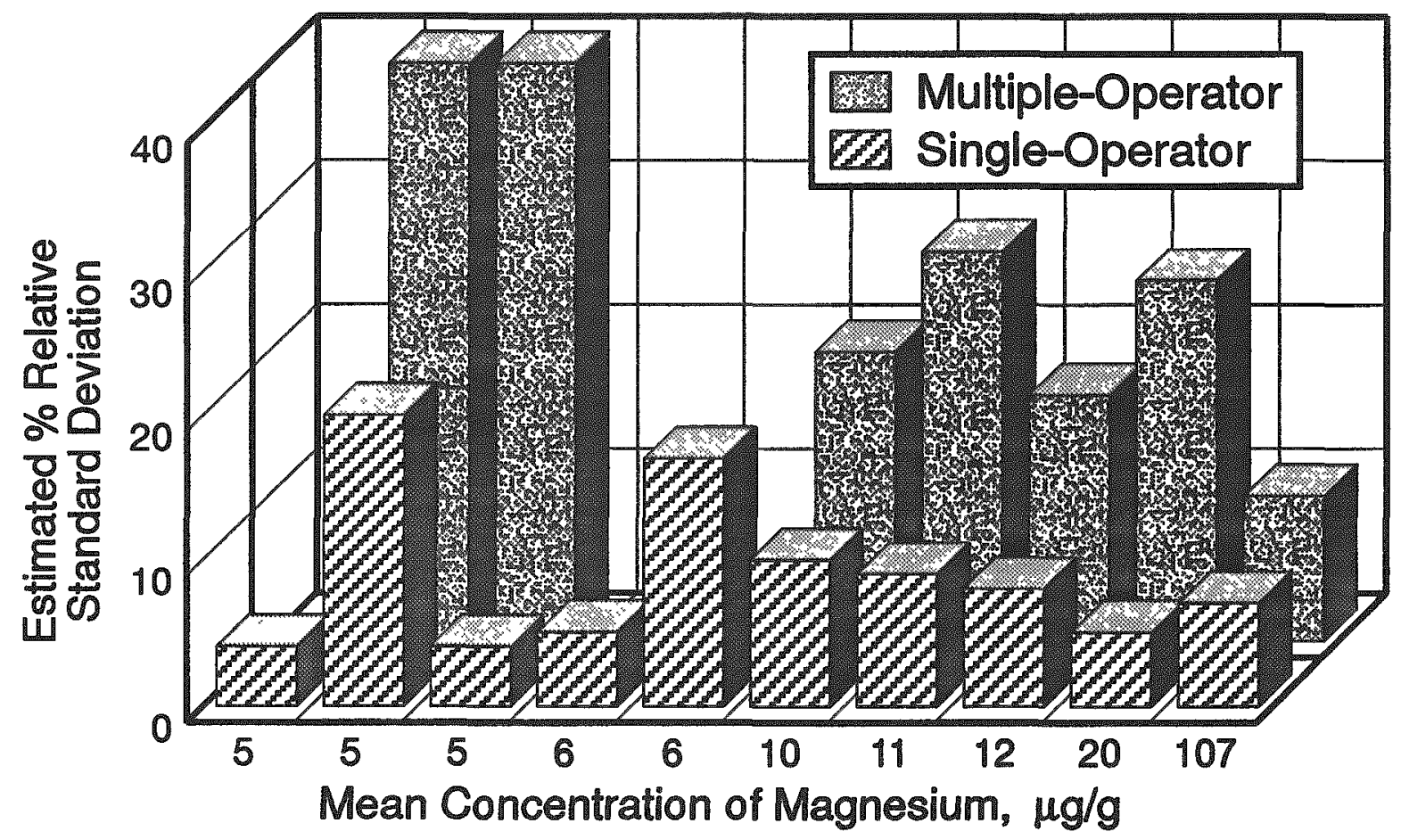

Figure 12. Estimated Precision of the Sequential Batch Extraction Method for Magnesium in the Composite Mining Waste

Extraction of Manganese from the composite Mining Waste. The multiplelaboratory precision of the sequential batch extraction method for extracting manganese from the composite mining waste can be estimated for only the first five extracts (Table 67). The same is also true for single-operator precision of the method (Table 82). Figure 13 is a plot of the estimated percent relative standard deviation of the extraction method versus mean concentration of manganese in the composite mining waste extract. The estimated multiple-laboratory percent relative standard deviation for the extraction procedure is high, averaging 348 over the concentration range, while the estimated single-operator percent relative standard deviation averages about $9 \%$. For the concentration range, 0.65 to $36.7 \mu \mathrm{g} / \mathrm{g}$, the estimated standard deviation of the extraction procedure appears to vary directly with the concentration of manganese extracted. 


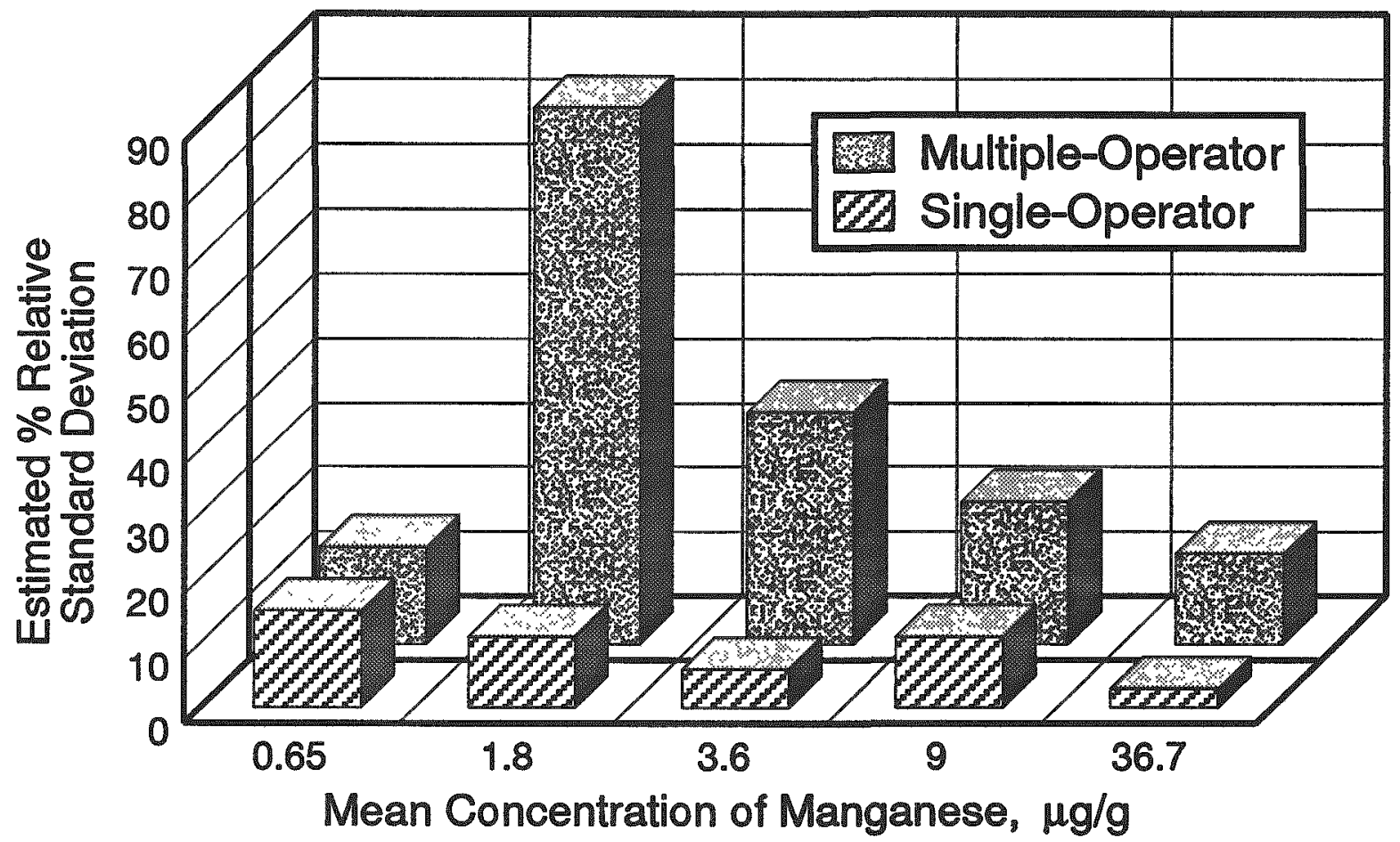

Figure 13. Estimated Precision of the Sequential Batch Extraction Method for Manganese in the Composite Mining Waste

Extraction of silicon from the composite Mining waste. The data used for estimating the precision of the sequential batch extraction method in extracting silicon from the composite mining waste are given in Tables 68 (multiple-laboratory) and 83 (single-operator). Figure 14 is a plot of the estimated percent relative standard deviation of the extraction method versus mean concentration of silicon in the composite mining waste extract. For the first five extracts containing concentrations of 20 to $55 \mu \mathrm{g} / \mathrm{g}$, the multiplelaboratory precision averages $22 \%$, and the single-operator precision averages about $7 \%$. The estimated single-operator percent relative standard deviation for the extraction method at extract concentrations of 14 and $15 \mu \mathrm{g} / \mathrm{g}$ is $0 \%$. The multiple-laboratory percent relative standard deviation for the extraction procedure is also estimated to be 0 of for three of the five extracts containing 14 and $15 \mu \mathrm{g}$ silicon/g composite mining waste. This increase in precision may be concentration dependent or due to an increased proficiency of the operator in performing the extraction procedure. For extraction of silicon, the estimated standard deviation of the extraction procedure appears to vary directly with silicon concentration over the range of 14 to $55 \mu \mathrm{g} / \mathrm{g}$.

Extraction of zinc from the composite Mining Waste. The multiplelaboratory precision of the sequential batch extraction method for extracting zinc from the composite mining waste can be estimated for only three of the composite mining waste extracts (Table 69). The same is true for singleoperator precision of the method (Table 84 ). The estimated percent relative standard deviation of the extraction method versus mean concentration of zinc in the composite mining waste extract is given in Figure 15. 


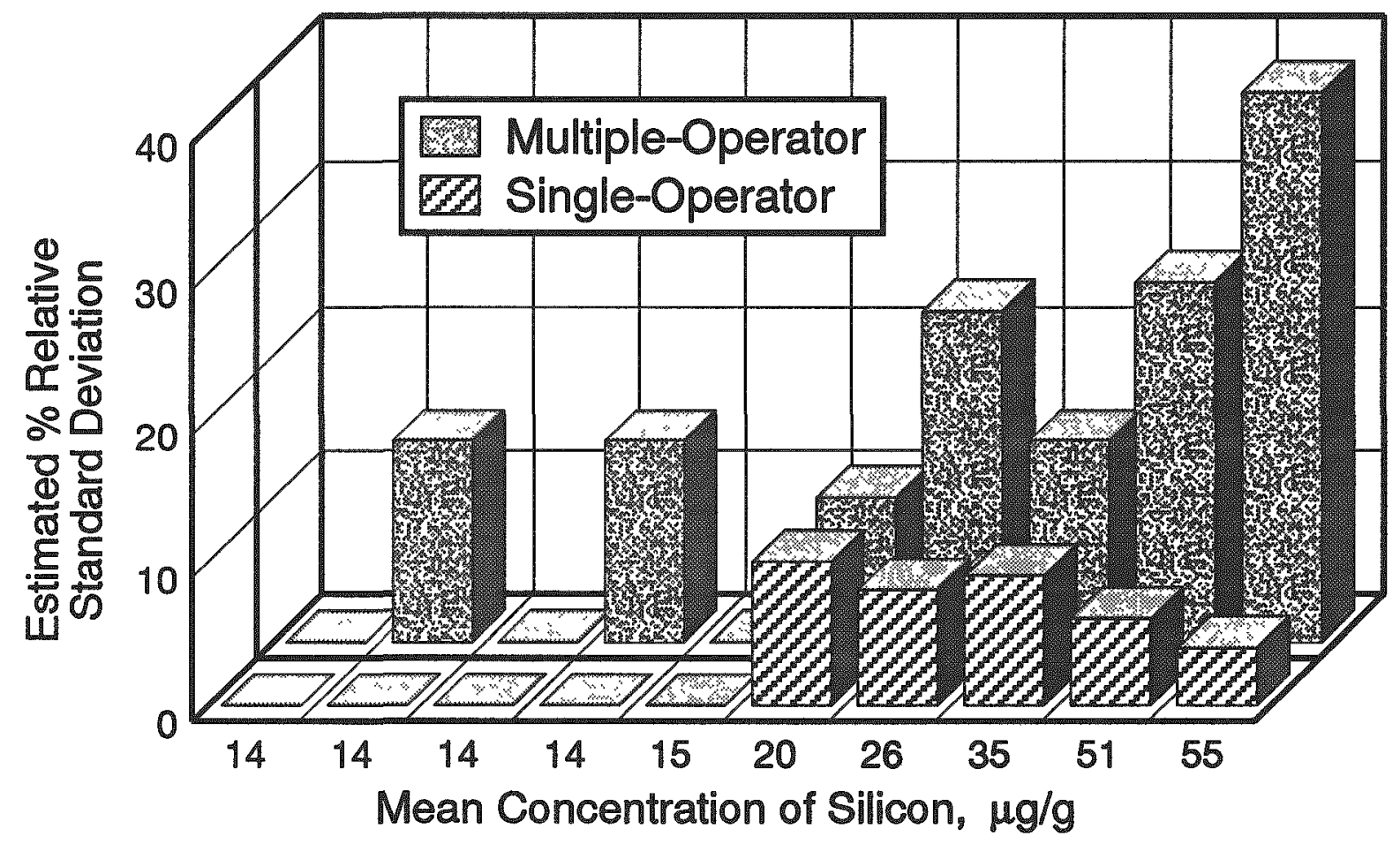

Figure 14. Estimated Precision of the Sequential Batch Extraction Method for Silicon in the Composite Mining Waste

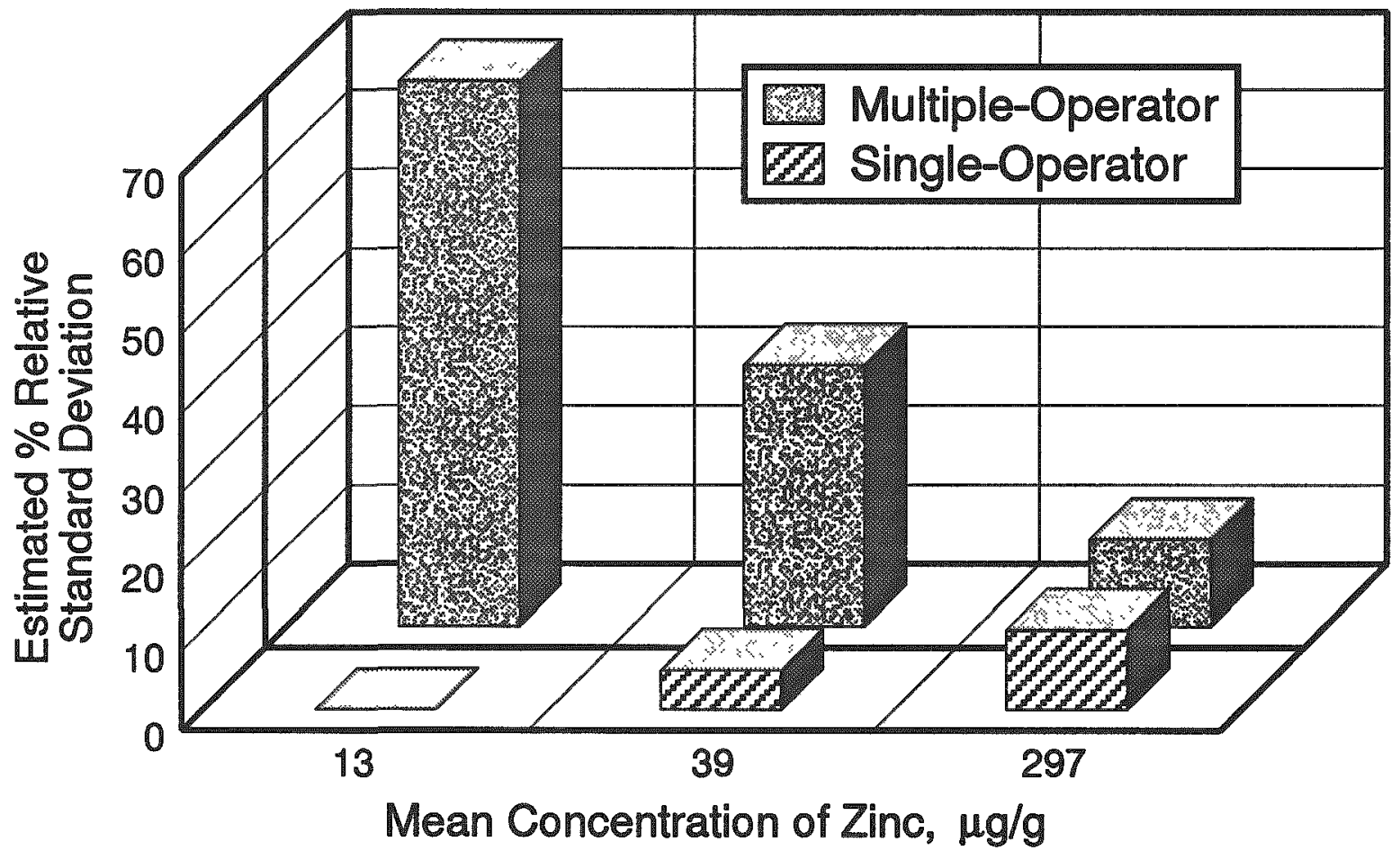

Figure 15. Estimated Precision of the Sequential Batch Extraction Method for Zinc in the Composite Mining Waste 
The precision of the extraction procedure may be concentration dependent. The limited data make discussion concerning the relationship between the concentration of zinc in the extract and the precision of the method difficult. However, it appears that the estimated standard deviation of the method for extracting $z$ inc may vary directly with concentration. The multiple-laboratory precision of the method for extracting zinc from the composite mining waste is low, especially at the concentration values of 13 and $39 \mu \mathrm{g} / \mathrm{g}$. The single-operator precision at these concentrations is much better than the multiple-operator precision.

The estimated percent relative standard deviation values for many of the elements having similar concentrations in the spray dryer waste extracts vary. These elements include aluminum at $25.5 \mu \mathrm{g} / \mathrm{g}$ and strontium at 25.4 $\mu \mathrm{g} / \mathrm{g}$ (Figures 1 and 8 ), barium at $41.3 \mu \mathrm{g} / \mathrm{g}$ and strontium at $42.7 \mu \mathrm{g} / \mathrm{g}$ (Figures 2 and 8 ), and sodium at $96 \mu \mathrm{g} / \mathrm{g}$ and strontium at $98 \mu \mathrm{g} / \mathrm{g}$ (Figures 7 and 8). The same is also true for the estimated percent relative standard deviation values for many of the elements having similar concentrations in the composite mining waste extracts. These elements include silicon at 35 $\mu \mathrm{g} / \mathrm{g}$ and $z$ inc at $39 \mu \mathrm{g} / \mathrm{g}$ (Figures 14 and 15 ), silicon at $20 \mu \mathrm{g} / \mathrm{g}$ and magnesium at $20 \mu \mathrm{g} / \mathrm{g}$ (Figures 14 and 12 ), silicon at $55 \mu \mathrm{g} / \mathrm{g}$ and lead at 63 $\mu \mathrm{g} / \mathrm{g}$ (Figures 14 and 11 ) and magnesium at 11 and $12 \mu \mathrm{g} / \mathrm{g}$ and $\mathrm{zinc}$ at $13 \mu \mathrm{g} / \mathrm{g}$ (Figures 12 and 15). These data indicate that the estimated precision of the sequential batch extraction method using acidic extraction fluid varies with the element of interest. In addition, the limited data for calcium and silicon, two common elements of interest for the spray dryer waste and the composite mining waste, show that for similar concentrations of these elements in the extracts, the estimated percent relative standard deviation values also vary (Figures 4, 6, 10, and 14). This indicates waste-specific effects on the precision of the extraction procedure.

For the materials tested in this study, the estimated multiple-laboratory precision of the extraction procedure for extracting many of the elements of interest varies somewhat with the elemental concentration in the extract, and in many cases, there is a direct relationship between estimated multiplelaboratory standard deviation and extract concentration. Only the data for extraction of silicon from the composite mining waste indicate a possible relationship between increased practice in performing the extraction procedure (later extract number) and increased precision.

For the elements of interest and their concentrations in the spray dryer waste, the estimated multiple-laboratory percent relative standard deviation ranges from 0 to $28 \%$, with the exception of barium, which has an estimated percent relative standard deviation ranging from 10 to $55 \%$. The estimated single-operator percent relative standard deviation values for extraction of the spray dryer waste elements are lower than the multiple-laboratory values. They range from 0 to 18\%, except for the boron values, which range from 6 to $29 \%$.

For the elements of interest and their concentrations in the composite mining waste, the estimated multiple-laboratory percent relative standard deviation ranges from 0 to $83 \%$. The estimated single-operator percent relative standard deviation values for extraction of the composite mining waste elements are much lower than the multiple-laboratory values. They range from 0 to $20 \%$. 
Evaluation of the Data from Analysis of the special Analytical samples

Final statistics, mean concentration and standard deviation values, resulting from statistical evaluation of the data from analysis of the special analytical samples are listed in Tables 40-54. These data were used to calculate the $95 \%$ confidence intervals for the elemental concentrations in the four filtrate types from the spray dryer waste and the composite mining waste. The mean concentration values and the $95 \%$ confidence intervals, which are listed in Tables 85-88, were evaluated to determine if:

1. The mean concentration for the $0.45-\mu \mathrm{m}$ filtrate falls within the $95 \%$ confidence interval for the $0.8-\mu \mathrm{m}$ filtrate, and the mean concentration for the $0.8-\mu \mathrm{m}$ filtrate falls within the $95 \%$ confidence interval for the $0.45-\mu \mathrm{m}$ filtrate.

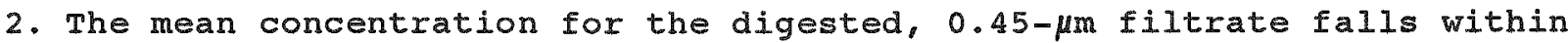

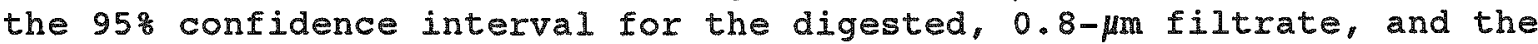

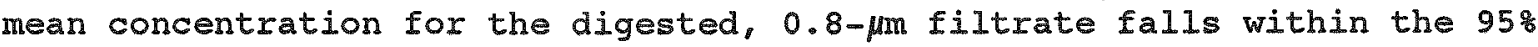
confidence interval for the digested, $0.45-\mu \mathrm{m}$ filtrate.

3. The mean concentration for the $0.45-\mu \mathrm{m}$ filtrate falls within the $95 \%$ confidence interval for the digested, $0.45-\mu \mathrm{m}$ filtrate, and the mean concentration for the digested, 0.45- $\mu \mathrm{m}$ filtrate falls within the $95 \%$ confidence interval for the $0.45-\mu \mathrm{m}$ filtrate.

4. The mean concentration for the $0.8-\mu m$ filtrate falls within the $95 \%$ confidence interval for the digested, $0.8-\mu \mathrm{m}$ filtrate, and the mean concentration for the digested, $0.8-\mu \mathrm{m}$ filtrate falls within the $95 \%$ confidence interval for the $0.8-\mu \mathrm{m}$ filtrate.

If the mean concentration values in each case fall within the 958 confidence intervals, the variable being evaluated, filter pore size or digestion versus nondigestion, is assumed to have no effect on the analytical concentration determined in the extract.

The mean concentration values and $95 \%$ confidence intervals for the concentrations of aluminum, barium, boron, and calcium in the spray dryer waste special analytical samples are given in Table 85 . For aluminum and boron, these data show that there is no difference in the concentrations of these elements in the $0.45-\mu \mathrm{m}$ extracts versus the $0.8-\mu \mathrm{m}$ extracts. There is also no difference between their concentrations in the digested versus nondigested extracts. In some cases, the mean concentration values fall just outside the $95 \%$ confidence intervals by one or two $\mu \mathrm{g} / \mathrm{g}$. Because the difference is so small, it is assumed that there is no effect. The mean concentration value for calcium in the nondigested, $0.45-\mu \mathrm{m}$ filtrate is high compared to the calcium concentration in the digested, $0.45-\mu \mathrm{m}$ filtrate. All other extracts have mean calcium concentrations falling within the $95 \%$ confidence intervals. The data for barium show that digestion of the spray dryer waste extracts greatly affects the barium concentration that is determined. For the comparisons involving digested samples, the digested extracts have much higher mean concentration values than the nondigested extracts, and the mean concentration values fall well above the corresponding confidence intervals. There is also a significant difference between the concentration of barium determined in the digested, $0.45-\mu \mathrm{m}$ filtrate $(75.3$ $\mu \mathrm{g} / \mathrm{g})$ and the digested, $0.8-\mu \mathrm{m}$ filtrate $(117 \mu \mathrm{g} / \mathrm{g})$. 
Table 85. Statistical Evaluation of the Data from Analysis of the spray Dryer Waste special Analytical samples for Aluminum, Barium, Boron, and Calciuna

\begin{tabular}{|c|c|c|c|c|}
\hline statistical values & Aluminum & Barium & Boron & Calcium \\
\hline $0.45-\mu \mathrm{m} \quad \overline{\mathrm{x}}^{\mathrm{b}}$ & 25 & 2.3 & 2.5 & 4,077 \\
\hline $0.8-\mu \mathrm{m} \bar{x}^{\mathrm{C}}$ & 24 & 2.6 & 2.6 & 3,981 \\
\hline $0.45-\mu \mathrm{m} 95 \%$ c.I. ${ }^{d}$ & $22-27$ & $1.5-3.0$ & $1.8-3.2$ & $3,624-4,530$ \\
\hline $0.8-\mu \mathrm{m} 95{ }^{\circ} \mathrm{C} . \mathrm{I}^{\mathrm{e}}$ & $21-26$ & $1.5-3.6$ & $2.3-2.9$ & $3,535-4,427$ \\
\hline $0.45-\mu \mathrm{m}-\mathrm{D}^{\mathrm{f}} \overline{\mathrm{X}}$ & 23 & 75.3 & 1.9 & 3,592 \\
\hline $0.8-\mu \mathrm{m}-\mathrm{D} \overline{\mathrm{x}}$ & 21 & 117 & 2.4 & 3,582 \\
\hline $\begin{array}{l}0.45-\mu \mathrm{m}-\mathrm{D} 95 \% \text { C.I. } \\
0.8-\mu \mathrm{m}-\mathrm{D} 958 \text { C.I. }\end{array}$ & $\begin{array}{l}22-24 \\
19-22\end{array}$ & $\begin{array}{l}69.7-80.9 \\
106-128\end{array}$ & $\begin{array}{l}1.4-2.4 \\
1.8-3.0\end{array}$ & $\begin{array}{l}3,130-4,054 \\
3,180-3,984\end{array}$ \\
\hline $\begin{array}{l}0.45-\mu \mathrm{m} \overline{\mathrm{x}} \\
0.45-\mu \mathrm{m}-\mathrm{D} \overline{\mathrm{x}} \\
0.45-\mu \mathrm{m} 95 \% \mathrm{C} . I . \\
0.45-\mu \mathrm{m}-\mathrm{D} 958 \mathrm{C} . \mathrm{I} .\end{array}$ & $\begin{array}{l}25 \\
23 \\
22-27 \\
22-24\end{array}$ & $\begin{array}{c}2 \cdot 3 \\
75 \cdot 3 \\
1.5-3.0 \\
69 \cdot 7-80.9\end{array}$ & $\begin{array}{c}2.5 \\
1.9 \\
1.8-3.2 \\
1.4-2.4\end{array}$ & $\begin{array}{c}4,077 \\
3,592 \\
3,624-4,530 \\
3,130-4,054\end{array}$ \\
\hline $\begin{array}{l}0.8-\mu \mathrm{m} \bar{x} \\
0.8-\mu \mathrm{m}-\mathrm{D} \overline{\mathrm{x}} \\
0.8-\mu \mathrm{m} 95 \% \mathrm{C} . I . \\
0.8-\mu \mathrm{m}-\mathrm{D} 95 \% \mathrm{C} . I .\end{array}$ & $\begin{array}{l}24 \\
21 \\
21-26 \\
19-22\end{array}$ & $\begin{array}{l}2 \cdot 6 \\
117 \\
1.5-3 \cdot 6 \\
106-128\end{array}$ & $\begin{array}{c}2.6 \\
2.4 \\
2.3-2.9 \\
1.8-3.0\end{array}$ & $\begin{array}{c}3,981 \\
3,582 \\
3,535-4,427 \\
3,180-3,984\end{array}$ \\
\hline
\end{tabular}

a Units are in $\mu g / g$.

b Mean concentration in the extract resulting from filtration using a $0.45-\mu \mathrm{m}$ pore-size filter

c Mean concentration in the extract resulting from filtration using a $0.8-\mu \mathrm{m}$ pore-size filter

d $95 \%$ confidence interval for the concentrations determined in the $0.45-\mu \mathrm{m}$ filtrates

- $95 \%$ confidence interval for the concentrations determined in the $0.8-\mu \mathrm{m}$ filtrates

f Digested extract

The mean concentration values and $95 \%$ confidence intervals for the concentrations of chromium, silicon, sodium, and strontium in the spray dryer waste special analytical samples are given in Table 86. For chromium, there is no difference in the concentration in the $0.45-\mu \mathrm{m}$ extract versus the $0.8-$ $\mu \mathrm{m}$ extract. There is also no difference between the chromium concentrations in the digested versus nondigested extracts of the spray dryer waste. For sodium and strontium, the concentrations determined in the nondigested, $0.8-$ $\mu \mathrm{m}$ filtrate are higher than those determined in the digested, $0.8-\mu \mathrm{m}$ filtrate, and the data for silicon show that there is a difference between the silicon concentrations determined in the digested extracts versus the nondigested extracts for both filter pore sizes. The silicon concentrations in the digested, $0.45-\mu \mathrm{m}$ extract and the digested, 0.8- $\mu \mathrm{m}$ extract are significantly lower than the silicon concentrations determined in the nondigested extracts. 
Table 86. Statistical Evaluation of the Data from Analysis of the spray Dryer Waste special Analytical samples for Chromium, silicon, Sodium, and strontium

\begin{tabular}{|c|c|c|c|c|}
\hline statistical values & Chromium & silicon & Sodium & strontium \\
\hline $\begin{array}{l}0.45-\mu \mathrm{m} \overline{\mathbf{x}}^{b} \\
0.8-\mu \mathrm{m} \bar{x}^{\mathrm{c}} \\
0.45-\mu \mathrm{m} 95 \% \text { C.I. }{ }^{d} \\
0.8-\mu \mathrm{m} 95 \% \text { C.I. }\end{array}$ & $\begin{array}{c}2.7 \\
2.6 \\
2.5-2.9 \\
2.4-2.8\end{array}$ & $\begin{array}{c}179 \\
175 \\
170-188 \\
168-182\end{array}$ & $\begin{array}{c}3,510 \\
3,471 \\
3,084-3,936 \\
3,045-3,897\end{array}$ & $\begin{array}{c}209 \\
208 \\
200-218 \\
199-217\end{array}$ \\
\hline 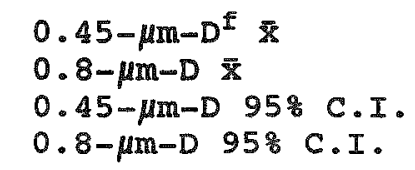 & $\begin{array}{c}2.6 \\
2.4 \\
2.5-2.7 \\
2.2-2.6\end{array}$ & $\begin{array}{c}138 \\
117 \\
126-150 \\
85-149\end{array}$ & $\begin{array}{c}3,132 \\
3,001 \\
2,735-3,529 \\
2,664-3,338\end{array}$ & $\begin{array}{c}199 \\
193 \\
196-202 \\
188-198\end{array}$ \\
\hline $\begin{array}{l}0.45-\mu \mathrm{m} \overline{\mathrm{x}} \\
0.45-\mu \mathrm{m}-\mathrm{D} \overline{\mathrm{s}} \\
0.45-\mu \mathrm{m} 958 \mathrm{C} . \mathrm{I} . \\
0.45-\mu \mathrm{m}-\mathrm{D} 958 \mathrm{C} . \mathrm{I} .\end{array}$ & $\begin{array}{c}2.7 \\
2.6 \\
2.5-2.9 \\
2.5-2.7\end{array}$ & $\begin{array}{c}179 \\
138 \\
170-188 \\
126-150\end{array}$ & $\begin{array}{c}3,510 \\
3,132 \\
3,084-3,936 \\
2,735-3,529\end{array}$ & $\begin{array}{c}209 \\
199 \\
200-218 \\
196-202\end{array}$ \\
\hline $\begin{array}{l}0.8-\mu \mathrm{m} \overline{\mathrm{x}} \\
0.8-\mu \mathrm{m}-\mathrm{D} \overline{\mathrm{x}} \\
0.8-\mu \mathrm{m} 95 \% \mathrm{C} . \mathrm{I} . \\
0.8-\mu \mathrm{m}-\mathrm{D} 95 \% \mathrm{C} . \mathrm{I} .\end{array}$ & $\begin{array}{c}2.6 \\
2.4 \\
2.4-2.8 \\
2.2-2.6\end{array}$ & $\begin{array}{c}175 \\
117 \\
168-182 \\
85-149\end{array}$ & $\begin{array}{c}3,471 \\
3,001 \\
3,045-3,897 \\
2,664-3,338\end{array}$ & $\begin{array}{c}208 \\
193 \\
199-217 \\
188-198\end{array}$ \\
\hline
\end{tabular}

a Units are in $\mu \mathrm{g} / \mathrm{g}$.

$b$ Mean concentration in the extract resulting from filtration using a $0.45-\mu \mathrm{m}$ pore-size filter

- Mean concentration in the extract resulting from filtration using a $0.8-\mu \mathrm{m}$ pore-size filter

d 95\% confidence interval for the concentrations determined in the $0.45-\mu m$ filtrates

- 95\% confidence interval for the concentrations determined in the $0.8-\mu \mathrm{m}$ filtrates

f Digested extract

The mean concentration values and $95 \%$ confidence intervals for the concentrations of barium, calcium, lead, and magnesium in the composite mining waste special analytical samples are given in Table 87 . The data for lead and magnesium show a slight difference between the concentrations of these elements determined in the $0.45-\mu \mathrm{m}$ extract versus the $0.8-\mu \mathrm{m}$ extract. The same is true for the concentrations of lead in the digested, $0.45-\mu \mathrm{m}$ extract and the digested, $0.8-\mu \mathrm{m}$ extract. For magnesium, there is a slight difference between the concentrations determined in the nondigested, 0.8- $\mu \mathrm{m}$ extract and the digested, 0.8- $\mu \mathrm{m}$ extract. For calcium, there is no difference in the concentration in the $0.45-\mu \mathrm{m}$ extract versus the $0.8-\mu \mathrm{m}$ extract. There is also no difference between the calcium concentrations in the digested versus nondigested extracts. The data for barium show that there is a difference between the barium concentrations determined in the digested, $0.45-\mu \mathrm{m}$ extract $(1.7 \mu \mathrm{g} / \mathrm{g})$ and the digested, $0.8-\mu \mathrm{m}$ extract $(4.4$ $\mu g / g)$ and between the barium concentrations determined in the nondigested, $0.8-\mu \mathrm{m}$ extract $(1.6 \mu \mathrm{g} / \mathrm{g})$ and the digested, $0.8-\mu \mathrm{m}$ extract $(4.4 \mu \mathrm{g} / \mathrm{g})$. 
Table 87. Statistical Evaluation of the Data from Analysis of the Composite Mining Waste Special Analytical Samples for Barium, Calcium, Lead, and Magnesium ${ }^{\mathrm{a}}$

\begin{tabular}{|c|c|c|c|c|}
\hline statistical values & Barium & Calcium & Lead & Magnesium \\
\hline $0.45-\mu \mathrm{m} \quad \bar{x}^{b}$ & 1.5 & 1,652 & 68 & 111 \\
\hline $0.8-\mu \mathrm{m} \overline{\mathrm{x}}^{\mathrm{c}}$ & 1.6 & 1,712 & 60 & 123 \\
\hline $0.45-\mu \mathrm{m} 958 \mathrm{C} . I^{d}$ & $1.3-1.7$ & $1,477-1,827$ & $63-73$ & $104-118$ \\
\hline $0.8-\mu \mathrm{m} 958 \mathrm{C} . I_{0}{ }^{\mathrm{a}}$ & $1.4-1.8$ & $1,519-1,905$ & $55-65$ & $115-131$ \\
\hline $0.45-\mu \mathrm{m}-D^{\mathrm{f}} \overline{\mathrm{x}}$ & 1.7 & 1,635 & 63 & 110 \\
\hline $0.8-\mu \mathrm{m}-\mathrm{D} \overline{\mathrm{x}}$ & 4.4 & 1,592 & 56 & 108 \\
\hline $0.45-\mu \mathrm{m}-\mathrm{D} 95 \div$ C.I. & $1.5-1.9$ & $1,449-1,821$ & $59-67$ & $102-118$ \\
\hline $0.8-\mu \mathrm{m}-\mathrm{D}$ 95\% C.I. & $3 \cdot 9-4.9$ & $1,407-1,777$ & $52-60$ & $101-115$ \\
\hline $0.45-\mu \mathrm{m} \overline{\mathrm{x}}$ & 1.5 & 1,652 & 68 & 111 \\
\hline $0.45-\mu \mathrm{m}-\mathrm{D} \overline{\mathrm{x}}$ & 1.7 & 1,635 & 63 & 110 \\
\hline $0.45-\mu \mathrm{m} 958$ C.I. & $1 \cdot 3-1 \cdot 7$ & $1,477-1827$ & $63-73$ & $104-118$ \\
\hline $0.45-\mu \mathrm{m}-D$ 95\% C.I. & $1.5-1.9$ & $1,449-1,821$ & $59-67$ & $102-118$ \\
\hline $0.8-\mu \mathrm{m} \overline{\mathrm{x}}$ & 1.6 & 1,712 & 60 & 123 \\
\hline $0.8-\mu \mathrm{m}-\mathrm{D} \overline{\mathrm{x}}$ & 4.4 & 1,592 & 56 & 108 \\
\hline $0.8-\mu \mathrm{m}$ 95응 C.I. & $1.4-1.8$ & $1,519-1,905$ & $55-65$ & $115-131$ \\
\hline $0.8-\mu \mathrm{m}-D$ 95\% C.I. & $3 \cdot 9-4 \cdot 9$ & $1,407-1,777$ & $52-60$ & $101-115$ \\
\hline
\end{tabular}

a Units are in $\mu \mathrm{g} / \mathrm{g}$.

b Mean concentration in the extract resulting from filtration using a $0.45-\mu m$ pore-size filter

c Mean concentration in the extract resulting from filtration using a $0.8-\mu \mathrm{m}$ pore-size filter

d $95 \%$ confidence interval for the concentrations determined in the $0.45-\mu \mathrm{m}$ filtrates

- $95 \%$ confidence interval for the concentrations determined in the $0.8-\mu \mathrm{m}$ filtrates

f Digested extract

The mean concentration values and $95 \%$ confidence intervals for the concentrations of manganese, silicon, and zinc in the composite mining waste special analytical samples are given in Table 88. The data for manganese show that there is no difference in the concentration in the $0.45-\mu \mathrm{m}$ extract

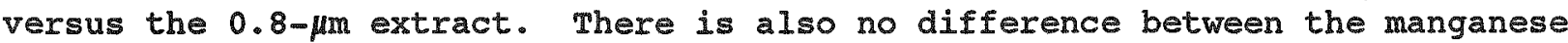
concentrations in the digested versus nondigested extracts. For both silicon and zinc, there is a difference between their concentrations in the $0.45-\mu \mathrm{m}$ extract and the $0.8-\mu \mathrm{m}$ extract. The concentration of silicon is higher in the $0.45-\mu \mathrm{m}$ extract than in the $0.8-\mu \mathrm{m}$ extract, while the concentration of zinc is lower in the $0.45-\mu \mathrm{m}$ extract. The data for zinc also show a higher zinc concentration $(366 \mu \mathrm{g} / \mathrm{g})$ in the nondigested, $0.8-\mu \mathrm{m}$ filtrate than in the digested, $0.8-\mu \mathrm{m}$ filtrate $(311 \mu \mathrm{g} / \mathrm{g})$. 
Table 88. Statistical Evaluation of the Data from Analysis of the Composite Mining Waste special Analytical samples for Manganese, silicon, and $\mathrm{zinc}^{\mathrm{a}}$

\begin{tabular}{|c|c|c|c|}
\hline Statistical Values & Manganese & silicon & Zinc \\
\hline $0.45-\mu \mathrm{m} \quad \bar{x}^{\mathrm{b}}$ & 36.2 & 67 & 298 \\
\hline $0.8-\mu \mathrm{m} \quad \bar{x}^{c}$ & 35.4 & 57 & 366 \\
\hline $0.45-\mu \mathrm{m} 95 \%$ C.I. ${ }^{d}$ & $33.2-39.2$ & $63-71$ & $273-323$ \\
\hline $0.8-\mu \mathrm{m} 95 \% \mathrm{C} . \mathrm{I}^{\mathrm{e}}$ & $32 \cdot 5-38 \cdot 3$ & $52-62$ & $334-398$ \\
\hline $0.45-\mu \mathrm{m}-\mathrm{D}^{\mathrm{F}} \overline{\mathrm{z}}$ & 34.2 & 63 & 298 \\
\hline $0.8-\mu \mathrm{m}-\mathrm{D} \overline{\mathrm{x}}$ & 33.0 & 49 & 311 \\
\hline $0.45-\mu \mathrm{m}-\mathrm{D} 95 \% \mathrm{C} . \mathrm{I}$. & $31.6-36.8$ & $56-70$ & $266-330$ \\
\hline $0.45-\mu \mathrm{m}-\mathrm{D} 958 \mathrm{C} . \mathrm{I}$. & $30.5-35.5$ & $42-56$ & $278-344$ \\
\hline $0.45-\mu \mathrm{m} \overline{\mathrm{x}}$ & 36.2 & 67 & 298 \\
\hline $0.45-\mu \mathrm{m}-\mathrm{D} \overline{\mathrm{x}}$ & 34.2 & 63 & 298 \\
\hline $0.45-\mu \mathrm{m} 95 \%$ C.I. & $33.2-39.2$ & $63-71$ & $273-323$ \\
\hline $0.45-\mu \mathrm{m}-\mathrm{D}$ 958 C.I. & $31.6-36.8$ & $56-70$ & $266-330$ \\
\hline $0.8-\mu \mathrm{m} \bar{z}$ & 35.4 & 57 & 366 \\
\hline $0.8-\mu \mathrm{m}-\mathrm{D} \overline{\mathrm{x}}$ & 33.0 & 49 & 311 \\
\hline $0.8-\mu \mathrm{m} 958 \mathrm{C} . I$. & $32 \cdot 5-38 \cdot 3$ & $52-62$ & $334-398$ \\
\hline $0.8-\mu \mathrm{m}-\mathrm{D} 958 \mathrm{C} . \mathrm{I}$ & $30.5-35.5$ & $42-56$ & $278-344$ \\
\hline
\end{tabular}

a Units are in $\mu \mathrm{g} / \mathrm{g}$.

b Mean concentration in the extract resulting from filtration using a $0.45-\mu \mathrm{m}$ pore-size filter

c Mean concentration in the extract resulting from filtration using a $0.8-\mu \mathrm{m}$ pore-size filter

d 958 confidence interval for the concentrations determined in the $0.45-\mu \mathrm{m}$ filtrates

- 958 confidence interval for the concentrations determined in the $0.8-\mu \mathrm{m}$ filtrates

Digested extract

Filter pore size, $0.45-\mu \mathrm{m}$ versus $0.8-\mu \mathrm{m}$, and digestion versus nondigestion affect certain elemental concentrations determined in the extracts of the spray dryer waste and composite mining waste. The effects of these variables are waste and element specific. For the spray dryer waste extracts, filter pore size and digestion versus nondigestion do not affect the concentrations of aluminum, boron, and chromium, and in the composite mining waste extracts, the concentrations of calcium and manganese are not affected. For calcium, sodium, and barium in the spray dryer waste and barium, magnesium, and zinc in the composite mining waste, the effects of digestion on the elemental concentrations determined in the extracts are influenced by the filter pore size used. The data for barium show that digestion of both the spray dryer waste extracts and composite mining waste extracts significantly affects the barium concentrations that are reported. 
Evaluating the Effects of Stopping the Sequential Batch Extraction Method over the Weekend

In planning the collaborative study, because some laboratories do not work over the weekend, it was decided that the extractions should be started on a Monday, stopped after filtering on Friday, and started again on the next Monday. This means that for those laboratories that followed the collaborative study specifications, \#1, 2, 3, 5, 7, and 8, extract 5 was generated after the solid was contacted with rinse solution in the extraction vessel over the weekend (longer than the six hours specified in section 9.7 of the method included as Appendix A). Laboratory \#6 did not follow the collaborative study specifications. This laboratory started the extractions on a Tuesday and continued them through the weekend. As a result, because of the difference in solid/rinse solution contact time between laboratory \#6 and the other laboratories prior to generating extract 5, the elemental concentrations in extract 5 generated by laboratory \#6 may vary from those in the fifth extracts generated by laboratories $\# 1,2,3,5,7$, and 8 .

To determine the effects of stopping the extraction procedure over the weekend, evaluations using the bias calculations given in ASTM Practice D2777 were performed. Although the bias calculations in ASTM Practice D2777 involve evaluating a known amount added versus a mean recovery, the calculations can be used to compare the mean concentration value determined by laboratory \#6 and the mean concentration value determined for the data generated by laboratories \#1, 2, 3, 5, 7, and 8 to determine if their difference is statistically significant at the 58 level.

These evaluations were performed for extracts one through five using final statistics data (Tables 10-24), which were generated using data from laboratories \#1, 2, 3, 5, 7, and 8, and laboratory \#6 data. Calculations to determine the significance of the difference between the two values at the $5 \%$ level were performed as specified for bias calculations in ASTM Practice D2777. Summaries of the data from these evaluations for the spray dryer waste extracts are given in Tables 89-93, and summaries of the data from the evaluations for the composite mining waste extracts are given in Tables 9498. The calculations involved evaluating the mean elemental concentrations in the laboratory $\# 6$ extracts and the mean concentrations, $\bar{x}$, from the final statistics for laboratories $\# 1,2,3,5,7$, and 8 to determine if a difference between the values exists at the $5 \%$ level.

Table 89. Evaluation of the Effects of stopping the sequential Batch Extraction Method Over the Weekend

Spray Dryer Waste: Extract 1

\begin{tabular}{|c|c|c|c|c|}
\hline Information & Aluminum & Barium & Calcium & sodium \\
\hline Lab \#6 Concentration, $\mu g / g$ & 32.0 & 49.9 & 4,382 & 4,007 \\
\hline $\begin{array}{l}\overline{\mathrm{x}} \text { from Labs \#1, } 2,3,5, \\
7,8 \text { Final statistics, } \mu \mathrm{g} / \mathrm{g}\end{array}$ & 25.5 & 14 & 3,755 & 3,515 \\
\hline \pm Difference & -6.5 & -35.9 & -627 & -492 \\
\hline \pm Difference & -20 & -72 & -14 & -12 \\
\hline $\begin{array}{l}\text { statistically significant } \\
\text { at the } 5: \text { Level? }\end{array}$ & yes & yes & yes & yes \\
\hline
\end{tabular}


Table 90. Evaluation of the fiects of stopping the sequential Batch Extraction Method over the Weekend Spray Dryer Waste: Extract 2

\begin{tabular}{|c|c|c|c|c|}
\hline Information & Aluminum & Barium & Calcium & sodium \\
\hline Lab \#6 concentration, $\mu \mathrm{g} / \mathrm{g}$ & 86.2 & 94.9 & 3,515 & 163 \\
\hline $\begin{array}{l}\overline{\mathrm{x}} \text { from Labs \#1, } 2,3,5, \\
7,8 \text { Final statistics, } \mu \mathrm{g} / \mathrm{g}\end{array}$ & 69.3 & 62 & 3,096 & 224 \\
\hline \pm Difference & -16.9 & -32.9 & -419 & +61 \\
\hline \pm 8 Difference & -20 & -35 & -12 & +37 \\
\hline $\begin{array}{l}\text { Statistically significant } \\
\text { at the } 5 \text { Level? }\end{array}$ & yes & yes & yes & yes \\
\hline
\end{tabular}

Table 91. Evaluation of the Effects of stopping the sequential Batch Extraction Method over the Weekend Spray Dryer Waste: Extract 3

\begin{tabular}{|c|c|c|c|c|}
\hline Information & Aluminum & Barium & Calcium & Sodium \\
\hline Lab \#6 Concentration, $\mu \mathrm{g} / \mathrm{g}$ & 130 & 64.3 & 3,403 & 54.5 \\
\hline $\begin{array}{l}\overline{\mathrm{x}} \text { from Labs \#1, } 2,3,5, \\
7,8 \text { Final statistics, } \mu \mathrm{g} / \mathrm{g}\end{array}$ & 108 & 45 & 2,795 & 100 \\
\hline \pm Difference & -22 & $-19 \cdot 3$ & -608 & +45.5 \\
\hline$\pm:$ Difference & -17 & -30 & -18 & +83 \\
\hline $\begin{array}{l}\text { statistically significant } \\
\text { at the } 5 \text { Level? }\end{array}$ & yes & yes & yes & yes \\
\hline
\end{tabular}

Table 92. Evaluation of the Effects of stopping the sequential Batch Extraction Method Over the Weekend Spray Dryer Waste: Extract 4

\begin{tabular}{|c|c|c|c|c|}
\hline Information & Aluminum & Barium & Calcium & sodium \\
\hline Lab \#6 Concentration, $\mu \mathrm{g} / \mathrm{g}$ & 160 & 48.9 & 2,914 & 38.5 \\
\hline $\begin{array}{l}\overline{\mathrm{x}} \text { from Labs \#1, } 2,3,5,5 \mathrm{~g} / \mathrm{g} \\
7,8 \text { Final statistics, } \mu \mathrm{g} / \mathrm{g}\end{array}$ & 128 & 38 & 2,529 & 96 \\
\hline \pm Difference & -32 & -10.9 & -385 & +57.5 \\
\hline$\pm \frac{8}{0}$ Difference & -20 & -22 & -13 & +149 \\
\hline $\begin{array}{l}\text { statistically significant } \\
\text { at the } 5: \text { Level? }\end{array}$ & yes & yes & yes & yes \\
\hline
\end{tabular}


Table 93. Evaluation of the Effects of stopping the sequential Batch Extraction Method over the Weekend

Spray Dryer Waste: Extract 5

\begin{tabular}{lcccc}
\hline Information & Aluminum & Barium & Calcium & Sodium \\
\hline Lab \#6 Concentration, $\mu \mathrm{g} / \mathrm{g}$ & 172 & 40.6 & 2,678 & 31.2 \\
F from Labs \#1, 2, 3, 5, & 145 & 41.3 & 2,301 & 98 \\
7,8 Final Statistics, $\mu \mathrm{g} / \mathrm{g}$ & & & & \\
\pm Difference & -27 & +0.7 & -377 & +66.8 \\
$\quad \pm 8$ Difference & -16 & +2 & -14 & +214 \\
$\begin{array}{l}\text { Statistically Significant } \\
\text { at the 5 : Level? }\end{array}$ & yes & no & yes & yes
\end{tabular}

Table 94. Evaluation of the Effects of stopping the sequential Batch Extraction Method over the Weekend Composite Mining Waste: Extract 1

\begin{tabular}{|c|c|c|c|c|}
\hline Information & Barium & Calcium & Magnesium & Manganese \\
\hline Lab \#6 Concentration, $\mu \mathrm{g} / \mathrm{g}$ & 1.8 & 1,925 & 110 & 40.0 \\
\hline $\begin{array}{l}\bar{x} \text { from Labs \#1, } 2,3,5, \\
7,8 \text { Final statistics, } \mu \mathrm{g} / \mathrm{g}\end{array}$ & 1.9 & 1,815 & 107 & 36.7 \\
\hline \pm Difference & +0.1 & -110 & -3 & -3.3 \\
\hline $\pm \%$ Difference & +6 & -6 & -3 & -8 \\
\hline $\begin{array}{l}\text { statistically significant } \\
\text { at the } 5 \% \text { Level? }\end{array}$ & no & no & no & no \\
\hline
\end{tabular}

Table 95. Evaluation of the Effects of stopping the sequential Batch Extraction Method Over the Weekend

Composite Mining Waste: Extract 2

\begin{tabular}{|c|c|c|c|c|}
\hline Information & Barium & Calcium & Magnesium & Manganese \\
\hline Lab \#6 Concentration, $\mu \mathrm{g} / \mathrm{g}$ & 3.5 & 622 & 18 & 8.1 \\
\hline $\begin{array}{l}\overline{\mathrm{x}} \text { from Labs \#1, } 2,3,5,8 \\
7,8 \text { Final statistics, } \mu \mathrm{g} / \mathrm{g}\end{array}$ & 3.2 & 628 & 20 & 9 \\
\hline \pm Difference & -0.3 & +6 & +2 & +0.9 \\
\hline \pm Difference & -9 & +1 & +11 & +11 \\
\hline $\begin{array}{l}\text { Statistically significant } \\
\text { at the } 5 \% \text { Level? }\end{array}$ & no & no & no & no \\
\hline
\end{tabular}


Table 96. Evaluation of the Efects of stopping the sequential Batch Extraction Method over the Weekend Composite Mining Waste: Extract 3

\begin{tabular}{|c|c|c|c|c|}
\hline Information & Barium & Calcium & Magnesium & Manganese \\
\hline Lab \#6 Concentration, $\mu \mathrm{g} / \mathrm{g}$ & 2.2 & 502 & 12 & 3.3 \\
\hline $\begin{array}{l}\bar{x} \text { from Labs \#1, } 2,3,5, \\
7,8 \text { Final statistics, } \mu \mathrm{g} / \mathrm{g}\end{array}$ & 2.3 & 448 & 12 & 3.6 \\
\hline \pm Difference & +0.1 & -54 & 0 & +0.3 \\
\hline \pm Difference & +5 & -11 & 0 & +9 \\
\hline $\begin{array}{l}\text { statistically significant } \\
\text { at the } 5 \& \text { Level? }\end{array}$ & no & yes & no & no \\
\hline
\end{tabular}

Table 97. Evaluation of the Ef fects of stopping the sequential Batch Extraction Method over the Weekend Composite Mining Waste: Extract 4

\begin{tabular}{lcccc}
\hline \multicolumn{1}{c}{ Information } & Barium & Calcium & Magnesium & Manganese \\
\hline Lab \#6 Concentration, $\mu \mathrm{g} / \mathrm{g}$ & 3.9 & 241 & 6.8 & 0.80 \\
$\begin{array}{l}\mathrm{X} \text { from Labs \#1, 2, 3,5, } \\
7,8 \text { Final Statistics, } \mu \mathrm{g} / \mathrm{g}\end{array}$ & 2.8 & 383 & 11 & 1.8 \\
\pm Difference & -1.1 & +142 & +4.2 & +1 \\
\pm D Difference & -28 & +59 & +6 & +125 \\
$\begin{array}{l}\text { Statistically Significant } \\
\text { at the 5\% Level? }\end{array}$ & yes & yes & yes & yes \\
\hline
\end{tabular}

Table 98. Evaluation of the Effects of stopping the sequential Batch Extraction Method over the Weekend Composite Mining Waste: Extract 5

\begin{tabular}{|c|c|c|c|c|}
\hline Information & Barium & Calcium & Magnesium & Manganese \\
\hline Lab \#6 Concentration, $\mu \mathrm{g} / \mathrm{g}$ & 12 & 251 & 6.3 & 0.40 \\
\hline $\begin{array}{l}\bar{x} \text { from Labs \#1, } 2,3,5, \\
7,8 \text { Final statistics, } \mu \mathrm{g} / \mathrm{g}\end{array}$ & 6 & 268 & 10 & 0.65 \\
\hline \pm Difference & -6 & +17 & +3.7 & +0.25 \\
\hline \pm Difference & -50 & +7 & +59 & +63 \\
\hline $\begin{array}{l}\text { statistically significant } \\
\text { at the } 5 \text { \& Level? }\end{array}$ & yes & yes & yes & yes \\
\hline
\end{tabular}


Based on the information given in Tables 89-93, the difference between laboratory \#6 concentrations and the final statistics $\bar{x}$ values is statistically significant for extracts one through five for all of the elements that can be evaluated, except for the concentrations of barium in the fifth extract. These data show that a factor other than the extended contact time between the spray dryer waste and rinse solution is responsible for the difference between the laboratory \#6 data and the final statistics mean concentration data. This is true because the difference is significant for not only the extract generated after the extended contact time, but also for those extracts generated after less than six hours of contact between the spray dryer waste and rinse solution.

It is also difficult to make any conclusions concerning the effects of stopping the procedure over the weekend on the elemental concentrations in the composite mining waste extracts. For extracts one and two (Tables 94 and $95)$, the difference between the laboratory \#6 data and the final statistics $\bar{x}$ data is not significant at the 5\% level. However, for extract three (Table 96). the difference is significant for calcium, and for extracts four and five (Tables 97 and 98), it is significant at the $5 \%$ level for all four elements that can be evaluated. Based on this information, it appears that a factor other than the extended contact time between the composite mining waste and rinse solution may also be responsible for the difference between the laboratory \#6 data and the final statistics mean concentration data.

Although no conclusions can be made concerning the effect of stopping the sequential batch extraction method over the weekend versus not stopping for the materials tested in this study, it is known that an effect is possible and may be specific to the material being tested and the $\mathrm{pH}$ of the extraction fluid. As a result, in section 9.7 of the new ASTM Method D5284-92 (Appendix F), the user is cautioned concerning the potential effect of solid/rinse solution storage, for longer than six hours, on the leachate characteristics.

Evaluation of the Control Data Versus Acidic Extraction Fluid Data

To generate baseline data for comparison with the data from the collaborative study, the spray dryer waste and composite mining waste were extracted using the sequential batch extraction method and deionized, distilled water as the extraction fluid. Average elemental concentrations in the water extracts were compared with the final statistics mean concentration values $(\bar{x})$ by substituting these data in the bias calculations given in ASTM Practice D2777. The data were evaluated for a difference at the $5 \%$ significance level. To reduce the bulkiness of this report, these comparisons are not presented; however, they are available in tabular form from Western Research Institute upon request.

The comparisons show that for the majority of the extracts one through ten, there is a statistical difference at the 5 : level between the concentrations of barium, boron, calcium, silicon, and sodium in the spray dryer waste/water extracts and the spray dryer waste/acid solution extracts. This is true for strontium in only four of the ten extracts. The difference between the strontium concentrations in the control extracts versus the acid solution extracts is not significant at the $5 \%$ level for extracts two, five, six, seven, eight, and nine. For aluminum, extracts one through five show significant difference between the control data and collaborative study data, while the difference for extracts six through ten is not significant at the 58 level. 
The data show that the leachability of the elements of interest in the spray dryer waste varies with the use of the dilute acid solution ( $\mathrm{pH}=4.3 \pm$ 0.05 ) versus deionized, distilled water. For aluminum, barium, boron, calcium, silicon, sodium, and strontium, the percent difference between the water extraction data and acidic extraction data varies among the ten extracts. For aluminum and boron, the difference between the water extraction data and acidic extraction data for the majority of extracts is negative (concentration in acid extract is less), and for calcium, sodium, and strontium, the majority of the differences is positive. For barium and silicon, the difference for approximately the first five extracts is positive and for the last extracts, it is negative.

The data show that for the majority of extracts one through ten, there is no statistical difference at the $5 \mathrm{q}$ significance level between the concentrations of barium, calcium, magnesium, and silicon in the composite mining waste/water extracts and the composite mining waste/acid solution extracts. However, for some of the extracts, the leachability of these elements from the composite mining waste does vary with the use of the dilute acid solution ( $\mathrm{pH}=5.0 \pm 0.05$ ) versus deionized, distilled water. Data for lead, manganese, and zinc are limited. Therefore, no conclusions concerning these elements can be made. For barium, calcium, magnesium, and silicon, the percent difference between the water extraction data and acidic extraction data varies among the ten extracts.

The average $\mathrm{pH}$ values of the extracts generated by extracting the spray dryer waste and the composite mining waste using the draft method with deionized, distilled water and the dilute acid solutions are listed in Tables 99 and 100. The $\mathrm{pH}$ values of the water extracts are generally very similar to the $\mathrm{pH}$ values of the dilute acid solution extracts.

Table 99. Average pH Values of control Extracts and collaborative study Extracts of the spray Dryer Waste

\begin{tabular}{lcccccccccc}
\hline Extract & 1 & 2 & 3 & 4 & 5 & 6 & 7 & 8 & 9 & 10 \\
\hline Control & 11.7 & 11.7 & 11.8 & 11.7 & 11.7 & 11.5 & 11.6 & 11.7 & 11.5 & 11.6 \\
Lab 1 & 11.6 & 11.4 & 11.2 & 11.4 & 11.3 & 11.3 & 11.2 & 11.3 & 11.1 & 11.0 \\
Lab 2 & 11.7 & 11.6 & 11.1 & 11.3 & 11.1 & 11.1 & 11.1 & 11.0 & 11.1 & 11.0 \\
Lab 3 & 11.5 & 11.3 & 11.2 & 11.1 & 11.0 & 11.1 & 10.9 & 10.8 & 10.9 & 10.8 \\
Lab 5 & $---^{\mathrm{a}}$ & $--^{\mathrm{a}}$ & $---^{\mathrm{a}}$ & $--^{\mathrm{a}}$ & $---^{\mathrm{a}}$ & $---^{\mathrm{a}}$ & $--^{\mathrm{a}}$ & $--^{\mathrm{a}}$ & $--^{\mathrm{a}}$ & 11.2 \\
Lab 7 & 11.3 & 10.8 & 10.6 & 10.5 & 10.3 & 10.5 & 10.2 & 10.1 & 10.0 & 10.5 \\
Lab 8 & 11.0 & 11.5 & 11.4 & 11.4 & 11.3 & 11.2 & 11.2 & 11.2 & 11.1 & 11.1 \\
\hline
\end{tabular}

a No measurement made 
Table 100. Average pH Values of Control Extracts and collaborative study Extracts of the Composite Mining Waste

\begin{tabular}{lcccccccccc}
\hline Extract & 1 & 2 & 3 & 4 & 5 & 6 & 7 & 8 & 9 & 10 \\
\hline Control & 6.6 & 6.7 & 7.2 & 7.5 & 8.0 & 8.6 & 8.5 & 8.5 & 8.6 & 8.5 \\
Lab 1 & 6.9 & 6.8 & 6.8 & 6.9 & 8.9 & 8.3 & 7.7 & 8.8 & 7.0 & 7.0 \\
Lab 2 & 6.1 & 7.1 & 7.4 & 8.2 & 8.2 & 8.4 & 8.7 & 8.4 & 8.5 & 8.6 \\
Lab 3 & 5.9 & 5.8 & 5.9 & 7.2 & 7.5 & 8.0 & 8.2 & 8.2 & 8.3 & 8.3 \\
Lab 5 & $--^{\mathrm{a}}$ & $--^{\mathrm{a}}$ & $--^{\mathrm{a}}$ & $--^{\mathrm{a}}$ & $--^{\mathrm{a}}$ & $--^{\mathrm{a}}$ & $--^{\mathrm{a}}$ & $--^{\mathrm{a}}$ & $--^{\mathrm{a}}$ & 7.9 \\
Lab 7 & 6.1 & 6.3 & 6.3 & 7.1 & 7.4 & 7.8 & 7.8 & 7.7 & 7.9 & 8.1 \\
Lab 8 & 6.8 & 6.2 & 7.4 & 7.2 & 7.5 & 8.7 & 8.0 & 8.2 & 8.1 & 8.0 \\
\hline
\end{tabular}

a No measurement made

Elemental solubility in sequential batch water extracts versus acid solution extracts depends on the solution chemistry of the extraction slurries. As a result, the effect of using an acidic extraction fluid versus water depends on the material being tested, the pH of the extraction fluid, the element or parameter(s) of interest, and the extraction number in the sequence of the sequential batch extraction.

\section{Analytical Bias Evaluations}

To evaluate the collaborative study analytical bias, the analytical mean concentration, $\bar{x}_{a}$, for each level of analytical standard, was compared to the actual elemental concentration used in preparing the analytical standard. The analytical mean concentration values were calculated using the collaborative study analytical data as specified in ASTM Practice D2777 and are listed in Tables 25-39. Calculations to determine if the analytical bias is significant at the $5 \%$ level were performed in accordance with AsTM Practice D2777. Summaries of the bias data for the spray dryer waste analytical standards are given in Tables 101-108. The elemental concentrations added to prepare the spray dryer waste standards, which are listed in Table 8 in units of $\mathrm{mg} / \mathrm{L}$, were converted to units of $\mu \mathrm{g} / \mathrm{g}$ and are listed as "amount added" in Tables 101-108. Summaries of the bias data for the composite mining waste analytical standards are given in Tables 109-115. The elemental concentrations added to prepare the composite mining waste standards, which are listed in Table 9 in units of $\mathrm{mg} / \mathrm{L}$, were also converted to units of $\mu \mathrm{g} / \mathrm{g}$ and are listed as "amount added" in Tables 109-115.

The analytical bias data, listed in Tables 101-108 for the dilute acid solution matrix having a $\mathrm{pH}$ of $4.3 \pm 0.05$, show that for aluminum, boron, and calcium (Tables 101, 103, and 104), the bias between the concentrations added to the analytical standards and the mean concentrations determined to be present in the standards by the collaborative study participants is not significant at the 5\% level. The same is true for the barium concentrations in the medium and high standards (Table 102). For silicon, sodium (low and 
medium standards), and strontium (Tables 106, 107, and 108), the bias between the collaborative study participants analytical standards data and the actual concentrations added is significant at the 5\% level. For silicon and sodium, the bias is positive and for strontium, the bias is negative. These data show that in the dilute acid solution matrix having a pH of $4.3 \pm 0.05$, the level of analytical accuracy for determining silicon, sodium, and strontium at the concentrations listed in Tables 106, 107 (low and medium standards), and 108, respectively, is not as good as desired based on the criterion recommended in ASTM Practice D2777. The same is also true for the concentration of chromium in the high concentration analytical standard listed in Table 105 and the concentration of barium in the low concentration standard, Table 102 .

Table 101. Determination of Analytical Bias for Aluminum in the Spray Dryer Waste Analytical standards

\begin{tabular}{|c|c|c|c|}
\hline Bias Information & Low standard & Medium standard & High standard \\
\hline Amount Added, $\mu g / g$ & 30 & 120 & 170 \\
\hline $\begin{array}{l}\bar{x}_{a} \text { (Labs \#1, 2, } 3,5, \\
7,8 \text { Final stat.) }, \mu g / g\end{array}$ & 29 & 118 & 172 \\
\hline \pm Bias & -1 & -2 & +2 \\
\hline \pm 8 Bias & -3 & -2 & +1 \\
\hline $\begin{array}{l}\text { Stat. significant } \\
\text { at the } 5 \% \text { Level? }\end{array}$ & no & no & no \\
\hline
\end{tabular}

Table 102. Determination of Analytical Bias for Barium in the Spray Dryer Waste Analytical standards

\begin{tabular}{lccc}
\hline Bias Information & Low Standard & Medium Standard & High Standard \\
\hline Amount Added, $\mu \mathrm{g} / \mathrm{g}$ & 5.0 & 30 & 50 \\
$\overline{\mathrm{X}}_{\mathrm{a}}($ Labs \#1, 2, 3, 5 & 7 & 31 & 50 \\
7 Final Stat.), $\mu \mathrm{g} / \mathrm{g}$ & & +1 & 0 \\
\pm Bias & +2 & +3 & 0 \\
\pm 8 Bias & +40 & no & no \\
Stat. Significant & yes & & \\
at the 5 Level? & & &
\end{tabular}


Table 103. Determination of Analytical Bias for Boron in the spray Dryer Waste Analytical standards

\begin{tabular}{|c|c|c|c|}
\hline Bias Information & Low standard & Medium standard & High standard \\
\hline Amount Added, $\mu \mathrm{g} / \mathrm{g}$ & 3.0 & 4 & 8 \\
\hline $\begin{array}{l}\overline{\mathrm{x}}_{\mathrm{a}}(\text { Labs } \# 1,2,3,5, \\
7,8 \text { Final stat.) }, \mu g / g\end{array}$ & 4 & 5 & 9 \\
\hline \pm Bias & +1 & +1 & +1 \\
\hline \pm Bias & +33 & +25 & +13 \\
\hline $\begin{array}{l}\text { Stat. Significant } \\
\text { at the } 5 \% \text { Level? }\end{array}$ & no & no & no \\
\hline
\end{tabular}

Table 104. Determination of Analytical Bias for Calcium in the Spray Dryer Waste Analytical standards

\begin{tabular}{|c|c|c|c|}
\hline Bias Information & Low standard & Medium standard & High Standard \\
\hline Amount Added, $\mu \mathrm{g} / \mathrm{g}$ & 2,000 & 2,600 & 4,000 \\
\hline $\begin{array}{l}\bar{x}_{\mathrm{a}} \text { (Labs \#1, } 2,3,5, \\
7,8 \text { Final Stat.) }, \mu \mathrm{g} / \mathrm{g}\end{array}$ & 1,983 & 2,611 & 3,927 \\
\hline \pm Bias & -17 & +11 & -73 \\
\hline \pm Bias & -1 & +0.4 & -2 \\
\hline $\begin{array}{l}\text { stat. Significant } \\
\text { at the } 5 \% \text { Level? }\end{array}$ & no & no & no \\
\hline
\end{tabular}

Table 105. Determination of Analytical Bias for Chromiun in the Spray Dryer Waste Analytical standards

\begin{tabular}{|c|c|c|c|c|}
\hline Bias Information & Low & standard & Medium standard & High Standard \\
\hline Amount Added, $\mu \mathrm{g} / \mathrm{g}$ & & 0.20 & 0.60 & 3.0 \\
\hline $\begin{array}{l}\overline{\mathrm{x}}_{\mathrm{a}} \text { (Labs \#1, 2, } 3,5, \\
7,8 \text { Final Stat.) }, \mu \mathrm{g} / \mathrm{g}\end{array}$ & & $--^{a}$ & $--^{a}$ & 2.8 \\
\hline \pm Bias & & $-\infty b$ & $---^{b}$ & -0.2 \\
\hline $\pm z$ Bias & & $-\infty b$ & $---^{b}$ & -7 \\
\hline $\begin{array}{l}\text { Stat. Significant } \\
\text { at the } 5 \% \text { Level? }\end{array}$ & & $--^{c}$ & $--^{c}$ & yes \\
\hline
\end{tabular}


Table 106. Determination of Analytical Bias for silicon in the Spray Dryer Waste Analytical standards

\begin{tabular}{|c|c|c|c|}
\hline Bias Information & Low standard & Medium Standard & High Standard \\
\hline Amount Added, $\mu \mathrm{g} / \mathrm{g}$ & 70 & 100 & 160 \\
\hline $\begin{array}{l}\bar{x}_{a}(\text { Labs \#1, } 2,3,5, \\
7,8 \text { Final Stat.) }, \mu g / g\end{array}$ & 75 & 107 & 171 \\
\hline \pm Bias & +5 & +7 & +11 \\
\hline $\pm z$ Bias & +7 & +7 & +7 \\
\hline $\begin{array}{l}\text { stat. Significant } \\
\text { at the } 58 \text { Level? }\end{array}$ & yes & yes & yes \\
\hline
\end{tabular}

Table 107. Determination of Analytical Bias for sodium in the Spray Dryer Waste Analytical standards

\begin{tabular}{|c|c|c|c|}
\hline Bias Information & Low standard & Medium standard & High standard \\
\hline Amount Added, $\mu \mathrm{g} / \mathrm{g}$ & 30 & 100 & 3,600 \\
\hline $\begin{array}{l}\overline{\mathrm{x}}_{\mathrm{a}} \text { (Labs \#1, } 2,3,5, \\
7,8 \text { Final Stat.), } \mu \mathrm{g} / \mathrm{g}\end{array}$ & 48 & 116 & 3,669 \\
\hline \pm Bias & +18 & +16 & +69 \\
\hline \pm 8 Bias & +60 & +16 & +2 \\
\hline $\begin{array}{l}\text { Stat. Significant } \\
\text { at the } 5 \% \text { Level? }\end{array}$ & yes & yes & no \\
\hline
\end{tabular}

Table 108. Determination of Analytical Bias for strontium in the Spray Dryer Waste Analytical standards

\begin{tabular}{lccc}
\hline Bias Information & Low Standard & Medium standard & High Standard \\
\hline Amount Added, $\mu \mathrm{g} / \mathrm{g}$ & 20 & 50 & 200 \\
$\overline{\mathrm{X}}_{\mathrm{a}}$ (Labs \#1, 2, 3, 5, 8 Final Stat.), $\mu \mathrm{g} / \mathrm{g}$ & 19 & 46.7 & 188 \\
\pm Bias & -1 & -3.3 & -12 \\
\pm Bias & -5 & -7 & -6 \\
Stat. Significant & yes & yes & yes \\
at the 5 Level? & & & \\
\hline
\end{tabular}


The analytical bias data, listed in Table 113 for the dilute acid solution matrix having a $\mathrm{pH}$ of $5.0 \pm 0.05$, show that for manganese, the bias between the concentrations added to the analytical standards and the mean concentrations determined to be present in the standards by the collaborative study participants is not significant at the 5\% level. The same is true for the barium concentrations in the medium and high standards (Table 109) and lead and magnesium concentrations in the low and high standards (Tables 111 and 112). For calcium (low and medium standards), silicon, and zinc (Tables 110, 114, and 115), the positive bias between the collaborative study participants' analytical standards data and the actual concentrations added is significant at the 58 level. This means in the dilute acid solution having a $\mathrm{pH}$ of $5.0 \pm 0.05$, the level of analytical accuracy for determining calcium, silicon, and zinc at the concentrations listed in Tables 110 (low and medium standards), 114 , and 115, respectively, is not as good as desired based on the criterion recommended in ASTM Practice D2777. The same is also true for the concentration of barium in the low concentration standard, Table 109; lead in the medium concentration standard, Table 111; and magnesium in the medium concentration standard, Table 112.

Table 109. Determination of Analytical Bias for Barium in the Composite Mining Waste Analytical standards

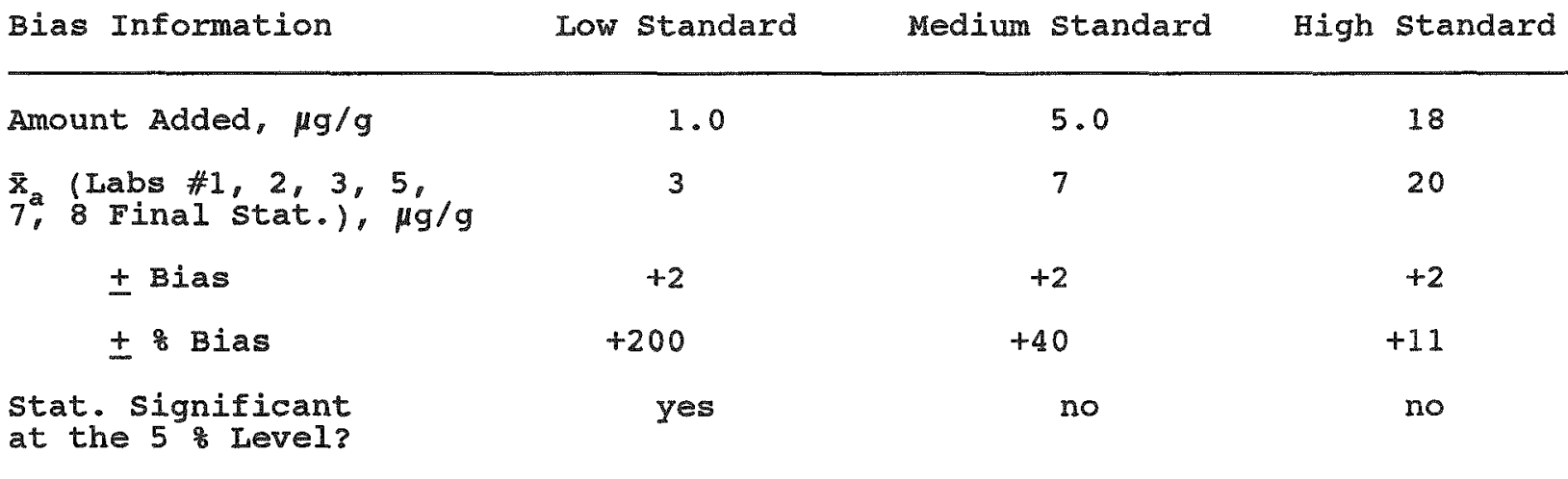

Table 110. Determination of Analytical Bias for Calcium in the Composite Mining Waste Analytical standards

Bias Information Low Standard Medium Standard High Standard

\begin{tabular}{lccc}
\hline Amount Added, $\mu \mathrm{g} / \mathrm{g}$ & 240 & 600 & 1,600 \\
$\overline{\mathrm{x}}_{\mathrm{a}}$ (Labs \#1, 2, 3, 5, & 248 & 622 & 1,614 \\
7 Final Stat.), $\mu \mathrm{g} / \mathrm{g}$ & & & +14 \\
\pm Bias & +8 & +22 & +1 \\
\% Bias & +3 & +4 & no \\
stat. Significant & yes & yes & \\
at the 5q Level? & & & \\
\hline
\end{tabular}


Table 111. Determination of Analytical Bias for Lead in the Composite Mining Waste Analytical standards

\begin{tabular}{|c|c|c|c|}
\hline Bias Information & Low standard & Medium standard & High standard \\
\hline Amount Added, $\mu g / g$ & 5.0 & 30 & 90 \\
\hline 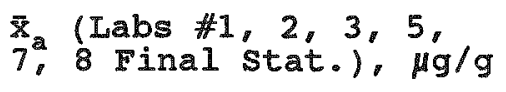 & 5 & 31 & 91 \\
\hline \pm Bias & 0 & +1 & +1 \\
\hline 8 Bias & 0 & +3 & +1 \\
\hline $\begin{array}{l}\text { Stat. Significant } \\
\text { at the } 5 \frac{\text { Level? }}{}\end{array}$ & no & yes & no \\
\hline
\end{tabular}

Table 112. Determination of Analytical Bias for Magnesium in the Composite Mining Waste Analytical standards

\begin{tabular}{|c|c|c|c|}
\hline Bias Information & Low standard & Medium standard & High standard \\
\hline Amount Added, $\mu \mathrm{g} / \mathrm{g}$ & 6.0 & 13 & 100 \\
\hline $\begin{array}{l}\bar{x}_{\mathrm{a}}(\text { Labs \#1, } 2,3,5,5 \\
7,8 \text { Final stat.) }, \mu \mathrm{g} / \mathrm{g}\end{array}$ & 6 & 14 & 102 \\
\hline \pm Bias & 0 & +1 & +2 \\
\hline : Bias & 0 & +8 & +2 \\
\hline $\begin{array}{l}\text { stat. Significant } \\
\text { at the } 5 \% \text { Level? }\end{array}$ & no & yes & no \\
\hline
\end{tabular}

Table 113. Determination of Analytical Bias for Manganese in the Composite Mining Waste Analytical standards

\begin{tabular}{|c|c|c|c|}
\hline Bias Information & Low standard & Medium Standard & High Standard \\
\hline Amount Added, $\mu \mathrm{g} / \mathrm{g}$ & 1.0 & 6.0 & 40 \\
\hline $\begin{array}{l}\bar{x}_{a}(\text { Labs \#1, 2, } 3,5, \\
7,8 \text { Final stat.), } \mu \mathrm{g} / \mathrm{g}\end{array}$ & 1.0 & 6.1 & 39.9 \\
\hline \pm Bias & 0 & +0.1 & -0.1 \\
\hline \& Bias & 0 & +2 & -0.3 \\
\hline $\begin{array}{l}\text { stat. Significant } \\
\text { at the } 5 \% \text { Level? }\end{array}$ & no & no & no \\
\hline
\end{tabular}


Table 114. Determination of Analytical Bias for silicon in the composite Mining Waste Analytical standards

\begin{tabular}{|c|c|c|c|c|}
\hline Bias Information & Low standard & Medium Standard & High & standard \\
\hline Amount Added, $\mu \mathrm{g} / \mathrm{g}$ & 10 & 30 & & 40 \\
\hline 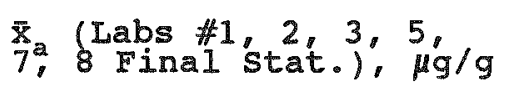 & 13 & 34 & & 44 \\
\hline \pm Bias & +3 & +4 & & +4 \\
\hline Bias & +30 & +13 & & +10 \\
\hline $\begin{array}{l}\text { Stat. Significant } \\
\text { at the } 5 \text { Level? }\end{array}$ & yes & yes & & yes \\
\hline
\end{tabular}

Table 115. Determination of Analytical Bias for zinc in the Composite Mining Waste Analytical standards

\begin{tabular}{|c|c|c|c|}
\hline Bias Information & Low standard & Medium Standard & High Standard \\
\hline Amount Added, $\mu \mathrm{g} / \mathrm{g}$ & 1.0 & 40 & 300 \\
\hline 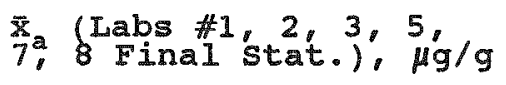 & 3.6 & 41.6 & 312 \\
\hline \pm Bias & +2.6 & +1.6 & +12 \\
\hline 8 Bias & +260 & +4 & +4 \\
\hline $\begin{array}{l}\text { stat. Significant } \\
\text { at the } 5 \frac{8}{8} \text { Level? }\end{array}$ & yes & yes & yes \\
\hline
\end{tabular}

\section{CONCLUSIONS}

The data generated in this study are specific to the test materials used in the study, the elements of interest, and the pH values of the extraction fluids used. For other materials, analytical parameters, and pH values, these data may not apply; however, based on the information obtained, the following conclusions can be made.

\section{Estimated Precision of the Extraction Procedure}

- The estimated precision of the sequential batch extraction method using acidic extraction fluid varies with the type of waste being tested and the element of interest.

- For the materials tested in this study, the estimated multiplelaboratory precision of the sequential batch extraction method for extracting many of the elements of interest varies somewhat with the elemental concentration extracted from the test material, and in many of these cases, there is a direct relationship between estimated multiple-laboratory standard deviation and extract concentration. 
- For most elements of interest in this study, the estimated precision of the extraction procedure varies with the extraction number in the sequence.

- For the test materials and elements of interest in this study, the estimated single-operator precision of the extraction method in many cases is much better than the estimated multiple-laboratory precision.

- For the elements of interest and their concentrations in the spray dryer waste, the estimated multiple-laboratory percent relative standard deviation ranges from 0 to $28 \%$, with the exception of barium, which has an estimated percent relative standard deviation ranging from 10 to 55\%. The estimated single-operator percent relative standard deviation values for extraction of the spray dryer waste elements are lower than the multiple-laboratory values. They range from 0 to 18\%, except for the boron values, which range from 6 to $29 \%$.

- For the elements of interest and their concentrations in the composite mining waste, the estimated multiple-laboratory percent relative standard deviation ranges from 0 to 838 . The estimated singleoperator percent relative standard deviation values for extraction of the composite mining waste elements are much lower than the multiplelaboratory values. They range from 0 to $20 \%$.

Effect of Filter Pore size and Digestion Versus Nondigestion on the Elemental Concentrations Determined in the Sequential Batch Extracts

- Filter pore size, $0.45-\mu \mathrm{m}$ versus $0.8-\mu \mathrm{m}$, and digestion versus nondigestion affect certain elemental concentrations determined in the extracts of the spray dryer waste and composite mining waste. The effects of these variables on the elemental concentrations are waste and element specific.

- For the spray dryer waste extracts, the concentrations of aluminum, boron, and chromium are not affected by the different filter pore sizes and digestion versus nondigestion. The same is true for the concentrations of calcium and manganese in the composite mining waste extracts.

- For calcium, sodium, and barium in the spray dryer waste extracts and barium, magnesium, and zinc in the composite mining waste extracts, the effects of digestion on the elemental concentrations determined in the extracts are influenced by the filter pore size used.

- Digestion of both the spray dryer waste extracts and composite mining waste extracts significantly affects the barium concentrations that are determined in these solutions.

\section{Effect of Stopping the Sequential Batch Extraction Method over the Weekend}

No conclusions can be made concerning the effect of stopping the sequential batch extraction method over the weekend versus not stopping for the materials tested in this study. However, it is known that an effect is possible and may be specific to the test material and the pH of the 
extraction fluid. As a result, in section 9.7 of the new ASTM Method D528492 (Appendix F), the user is cautioned concerning the potential effect of solid/rinse solution storage, for longer than six hours, on the leachate characteristics.

\section{Control Data Versus Acidic Extraction Fluid Data}

- Elemental solubility in sequential batch water extracts versus acid solution extracts depends on the solution chemistry of the extraction slurries. As a result, the effect of using an acidic extraction fluid versus water depends on the material being tested, the pH of the extraction fluid, the element or parameter(s) of interest, and the extraction number in the sequence of the sequential batch extraction.

- Using the test parameters of the sequential batch extraction method, the leachability of aluminum, barium, boron, calcium, silicon, sodium, and strontium from the spray dryer waste varies with the use of deionized, distilled water and the dilute acid solution having a pH of $4.3 \pm 0.05$.

- Under the test conditions of the sequential batch extraction method, the leachability of barium, calcium, magnesium, and silicon from the composite mining waste varies with the use of deionized, distilled water versus the dilute acid solution having a pH of $5.0 \pm 0.05$ for some of the extracts.

\section{Analytical Bias}

- In the collaborative study, the level of analytical accuracy for determining certain concentrations of silicon, sodium, strontium, chromium, and barium in the dilute acid solution standards (prepared using 60/40 wt sulfuric/nitric acid solution) having a pH of $4.3 \pm$ 0.05 was not as good as desired based on the criterion given in ASTM Practice D2777. For the levels of aluminum, boron, and calcium in these standards, the analytical accuracy was acceptable based on the ASTM Practice D2777 criterion.

- In the collaborative study, the level of analytical accuracy for determining certain concentrations of calcium, silicon, zinc, barium, lead, and magnesium in the dilute acid solution standards having a $\mathrm{pH}$ of $5.0 \pm 0.05$ was not as good as desired based on the criterion given in ASTM Practice D2777. For the manganese levels in these standards, the analytical accuracy was acceptable based on the same criterion.

\section{PRECISION AND BIAS STATEMENTS TO BE ADDED TO ASTM METHOD D5284-92}

Based on the data generated by the collaborative study, precision and bias statements and information obtained from the study were prepared for addition to ASTM Method D5284-92. These were balloted concurrently within ASTM Subcomittee D34.02 on Physical and Chemical Characterization and ASTM Main Committee D34 on Waste Management as a modification to the method. The precision and bias statements passed subcommittee balloting with a voting result of 48 affirmative votes, zero negative votes, and 67 abstentions. 
At the main committee level, there were 120 affirmative votes, zero negative votes, and 202 abstentions for the proposed addition. The precision and bias statements, which are included as Appendix $G$, will be balloted within the ASTM Society in August 1993. Society approval is expected, and precision and bias statements should be published as part of ASTM Method D5284-92 in 1994 .

Because the data generated in this study are specific to the test materials and elements evaluated in the study and may not apply to other materials, general precision statements that cover all wastes, analytical parameters, and extraction fluid pH values can not be prepared. However, the data generated in this study give the user information on both the multiplelaboratory and single-operator estimated precision of the extraction procedure when it is applied to two different waste materials using two different extraction fluid $\mathrm{pH}$ values. As a result, the precision statement for this method provides information on the variability that can be expected between test results when the method is used by one or more laboratories. This information will help potential users assess the usefulness of the method in their proposed applications. The data also provide information to aid the user in making decisions concerning filtering the extraction slurries and analyzing the extracts.

The bias of the extraction method can not be determined because no standard reference material exists. However, information on the analytical bias determined in the collaborative study will be added to the method, along with information on the comparative data evaluation for control data versus acidic extraction fluid data.

\section{RECOMMENDATIONS}

This standard method addresses the sequential release of certain constituents from waste materials using extraction fluid pH that reflects the $\mathrm{pH}$ of acidic precipitation in the geographic region where the waste is to be disposed. There are other factors that require consideration. These include the depositional environment, the chemistry of the waste-type, and the chemical interaction of the extraction fluid with the waste material. Geochemical factors, such as the composition and chemical characteristics of the soil and overburden at the disposal site affect leaching conditions and the resulting chemistry of the leachate. Chemical composition of the waste and the effects of the depositional environment and long-term leaching on reduction-oxidation elements, such as chromium, manganese, and iron, are also important. Chemical components of specific waste-types require investigation. Information concerning these factors and their interactions should be obtained and used in the development of future ASTM standard test methods, addressing multiple disposal conditions.

Analysis of the special analytical samples in this study showed specific differences in analytical values obtained using $0.45-\mu \mathrm{m}$ versus $0.8-\mu \mathrm{m}$ filter pore sizes and digestion versus nondigestion. In addition, the analytical accuracy for many of the elements in the extracts was not as good as desired. Accurate analytical methods for analyzing extracts generated by extraction procedures are very important. Results from this study show possible problems with the analytical methods that were used. These should be investigated. There is also a need for further evaluation of the effects of filter pore size on the elemental concentrations determined in extracts and the use of digestion as an analytical technique for certain elements. 
The author expresses thanks to the U.S. Department of Energy (DOE) for funding this work under Cooperative Agreement Number DE-FC21-86MC11076. The author also thanks the American Mining Congress, Environment Canada, and Amax Mining Company for jointly sponsoring this research with DOE. The voluntary participation of the laboratories in the collaborative study is also greatly appreciated, as well as, the project support given by AsTM's Institute for standards Research.

\section{DISCLAIMER}

Mention of specific brand names or models of equipment is for information only and does not imply endorsement of any particular brand. 
American Society for Testing and Materials, 1991, D2777-86, standard Practice for Determination of Precision and Bias of Applicable Methods of Committee D-19 on water. Annual Book of ASTM standards, 11.01: $31-44$.

Anerican Society for Testing and Materials, 1992, D3987-85, Standard Test Method for shake Extraction of solid Waste with Water. Annual Book of ASTM standards, $11.04: 37-40$.

American society for Testing and Materials, 1992, D4793-88, standard Test Method for sequential Batch Extraction of Waste with Water. Annual Book of AsTM standards, 11.04: 139-147.

California Environmental Protection Agency, 1991, Waste Extraction Test (WEI) Procedures. Register 91, No. 22, pgs. 669-672.

Canadian General standard Board, 1987, Leachate Extraction Procedure. 164GP-1 MP.

Environment Canada Federal/Provincial Research and Monitoring and Coordination Committee, 1990, The 1990 Canadian Long-Range Transport of Air Pollutants and Acid Deposition Assessment Report. Atmospheric Sciences, Part 3 .

Gordon, G.E., 1987, A Decade of Acid Rain Research. The Chemistry of Acid Rain, ACS symposium Series 349, American Chemical society, Washington, DC, 2-9.

Guttman, I., S.S. Wilks, and J.S. Hunter, 1971, Introductory Engineering statistics, J. Wiley and Sons: New York, NY.

Hidy, G.M., 1987, Subcontinental Air Pollution Phenomena. The Chemistry of Acid Rain, ACS symposium series 349, American chemical society, Washington, DC, $10-27$.

Resource Conservation and Recovery Act, 1984, 3001(g), 42 U.S.C. 6921(g).

U.S. EPA, 1980, Extraction Procedure Toxicity characteristic. Federal Reqister, May 19,45: 33063-33285.

U.S. EPA, 1990, Method 1312: Synthetic Precipitation Leaching Procedure. Test Methods for Evaluating solid Waste: Physical/Chemical Methods (SW846), Vol. 1C, 3rd Ed.

U.S. EPA, 1990, Method 1320: Multiple Extraction Procedure. Test Methods for Evaluating solid Waste: Physical/Chemical Methods (sw846), Vol. 1C, 3rd Ed.

U.S. EPA, 1990, Method 3010: Acid Digestion of Aqueous Samples and Extracts for Total Metals for Analysis by Flame Atomic Absorption spectroscopy or Inductively Coupled Plasma spectroscopy. Test Methods for Evaluating Solid Waste: Physical/Chemical Methods (SW846), Vol. 1A, 3rd Ed. 
U.S. EPA, 1990, Method 3050: Acid Digestion of Sediments, Sludges, and Soils. Test Methods for Evaluating solid Waste: Physical/Chemical Methods $(\mathrm{SW846)}, \mathrm{VOI}$. 1A, 3rd Ed.

U.S. EPA, 1990, Method 6010: Inductively Coupled Plasma Atomic Emission spectroscopy. Test Methods for Evaluating solid Waste: Physical/ Chemical Methods (SW846), Vol. 1A, 3rd Ed.

U.S. EPA, 1990, Toxicity Characteristic Leaching Procedure. Federal Register, March 29, 55: 11798-11877. 
APPENDIX A

Test Method Used by the Participants in the collaborative study 
-

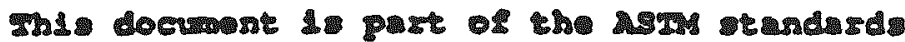

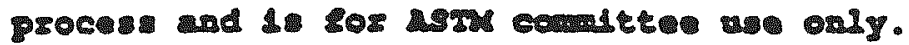

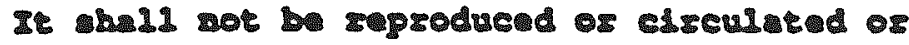

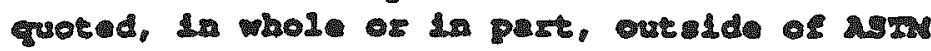

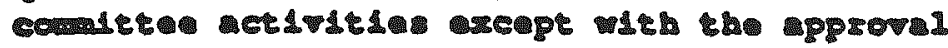

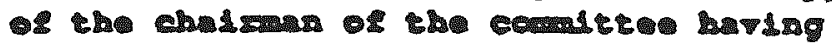

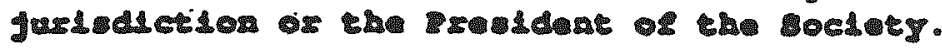

Docurase:

ac:

5is Grou: 03.02 .01 autbor:
234.02-044R

14an

\section{2. eope}

1.1 Ind test method is proceduxe for the gequentil 1 leaching of wate containing least five dry soldds to generate solutions to be used to determine the constituents leached under the apecified testing conditions.

1.2 This test mothod calls for the shaking of known weight of wate with acidic extraction Lluid of specified compostion and the separation of the idguid phase for andysi. The prof of the extraction fluid is to reflect the pH of acidic precipltation in the geographic xegion whexe the wate being tested is to be disposed. Ihe procedure Is conducted ten thmes in sequence on tre ame smple of wate. and gererates ten solutions.

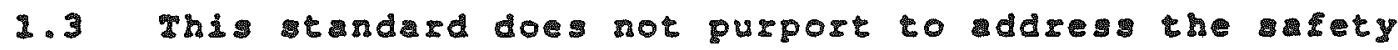
problens mociated with its uge. It is the responsibility of the user of this tandard to atabish apropricte arety and helth practice and determine the pplicability of regularory linitation prior to use.

IThis test method is under the juylsdiction of ASIM Comattee D-34 on Mate Mnagement and is the direct responglbility of subcornitte D34.02 on Rhysical and Cherical Characterimation. 


\section{Veferexced Document}

2.1 ASTM Standards:

- 75 Iractice for Sanpling Aggregetes ${ }^{2}$

D 20 gractice for Investigating and sampling of Soid and Rock for Engineering 8 urposes ${ }^{3}$

D 653 standard Terminology Relating to soli, Nock, and Contalned Fludas ${ }^{3}$

- 1229 Defindtions of Jerms Rejating to Mater

D 1193 Specification for Reagent water

D 2216 Method Lor Laboritory Determination of vatex (Molsture)

Contert of Sold, Rock, and SoLl-Aggregate Mixtures ${ }^{3}$

- 2234 Metrod for collection of aros sample of conls

- 2777 zractice for Determination of Precision and Bias of Applicable Methods of Coranittee D-19 on water

- 3370 zractices for Sampling water

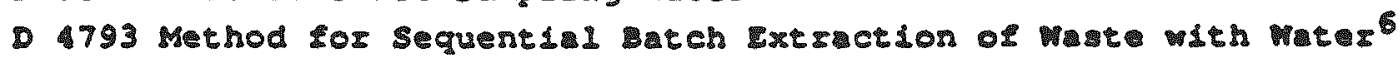

3. Sexposogy

3.1 Definitions:

3.1.1 For definitions of terms used in this tegt nethod, aec Definition D 1129.

3.2 symbols:

3.2.1 Varibles listed in this test method re defined in the individus sections where they re discussed. A 11 t ot defined variables is also given in section 11.

2Arrual Book of ASIM Stardards, Vol 04.03.

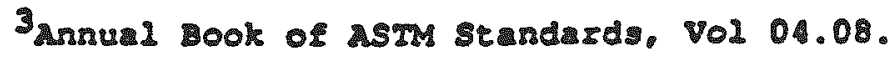
Ansur l sook of Asm Standards, V01 11.01.

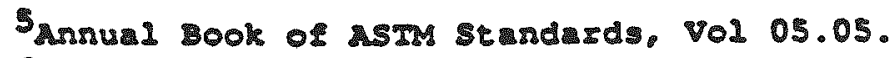
Grrnual Book of ASTM standards. Vol 11.01.

7Ansual Book of ASTM Standards, Vol 14.02. 


\section{Sigrasteance and 0 se}

4.1 Thls test method is dntended a mean som obtaining acguential extract of wate. Ihe extracts may be used to estinate the release of eertin constituents of the waste under the laboratory conditions described In this test method.

-.2 The ph of the extraction IIuId used in this tegt method 1 to reflect the prof ocidic precipitation in the geographic region whe the wate bing terted is to be disposed. Mensures should be takn to engure that an cerrate ph for the geographle reglon of interest \& used.

MOTE 1-FOF aequential batch extraction of wate using nonacidic axtraction fluid se referenced docunent D 1793 method for sequentil I Batch extraction of Waste with water.

4.3 An intent of this test method is that the sind pH of each of the extracts reflects the interaction of the extractant with the buffering capacity of the waste.

1. This test method is not intended to protide extract that re representative of the actul leachate produced from mate in the fleld or to produce extract to be usd as the ole ba of enginesing design.

4.5 This test method has not been demongtrated to simulate actual disposal site leaching conditioss.

4.6 Jhis test method produces extracto that are amenable to the determination of both mojor and minor (trace) constituents. When minor constituent are being determined, Lt is especially lmportant that precautions me takn in ample morage and handing to avold possible contamination of the amples.

1.7 Thi test method ha ben tested to determine it applicability to certain inorganic components in the uaste. This test aethod has not been tested for applicability to organic mubstances and rolatile matter (see 5.22 and 5.22 .1 ).

4. The gitation technique, rate, liquid-to-solid satio, ma Iiltration conditions specified in the prosedure may not be sutrable for extracting all types of wates lsee sections 7 , B. and the discussion in Apperdix $x 1$ ). 
- gpscas

5.1 straight edge (rueh a thin-edged yod atick).

5.2 Impermeable sheet of glized paper. 11 cloth. or other

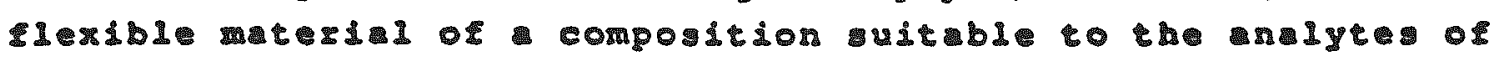
Interest.

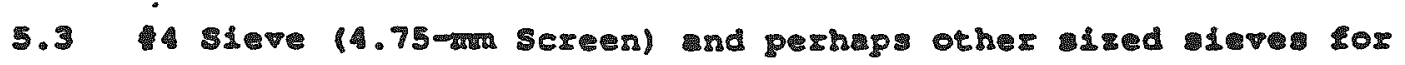
use In Section 2.2 .2 .

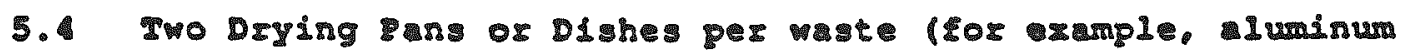

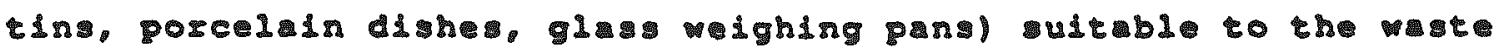
being tested and the Instructions given in 9.2 .

5.5 Drying oven. ny chemosticaly controlled drysng oven capable of maintining cemperature of $12^{\circ} \mathrm{C} \pm 5^{\circ} \mathrm{C}$ i acceptable

5.6 Descertor having capacity co hold the drying pan described in 5.4 and the crucibles described in 5.17 .

5.7 Imboratory sance capable of ughing to 0.19.

5. Exlemeyer jask, 2-l capacity. equipped with anetie tis bax.

5.9 Magnetie stir Pate.

5.10 Graduated CyIinder, 1-I or 2-L capacity. 
5.11 iper, 1-nil capacity.

5.12 Volunetric Flask, 1-I capacity.

5.13 ipet, 10-mi capacity (vaxious other siged pipets, including micropipets, my be necesary for section 9.3.2).

5.14 pH Meter, any pH aeter wh readablity of 0.01 unit and an aceuracy of \pm 0.05 units at $25^{\circ} \mathrm{C} 1 \mathrm{~s}$ acceptable.

5.15 Carboy-type container with spjgot. 20 to 50-L capacty. of composition suttable to the nature of the anyses to be performed.

\subsection{Large Glss Fussed.}

5.27 Two crucibles per mste, porcelain, 20-ml capacity each.

5.18 Andytical Balance capable of weighing to $0.1 \mathrm{mg}$.

5.29 Wash sottle, 500-mi capacity.

5.20 Agitation Eguipment, of any type that rotates the extraction the axis of rotation is horizontal and it goes through the center of the bottle, (see Figure 2 and discussion of agitation In Appendix $\mathrm{KI}$.

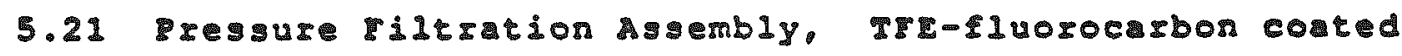
stainless teel pressure device guipped with $0.45-\mu m$ or $0.8-\mu m$ pore size fiter (see NOTE 6 , pertaining to section 9.5 ). 
5.22 Extzaction Vessis, cylisdici, wde-mouth, of composition altable to the nature of the mte and analyses to be pertormed, constructed of materils that wil not llow orption of constituents of dnterest, and sturdy enough to withatand the impact of the faling sample fragment. Contine size hould be selected so that the mple plus extraction fluid oceupy approximately 95 of the contalnex. Contalners must bave water-tght closur. Containers sor anples here gases by belessed hould bo provided with renting

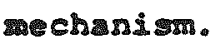

MOTE 2-The venting of the container the potential to effect the concentration of polatile compounds in the extract.

5.22.1 Extraction vessels should be cleaned in manner consistent with the ralyses to be pexformed. See Practice D3370, Section 13.

\section{Regert}

6.1 Ruxity of Reagents-oreagent grade chemical shall be used in 11 tests. Unles otherwise Indicated. It 1 intended that ald reagents hall conform to the specification of the Anerican chemical society. where such specifications are vailuble." other grades may be used, provided it is isgt ascertined that the reagent is of ufficienty high pusity to pesmit its use ithout lesaning the accuracy of the determination.

6.2. Burity of Watermunless otherwise indicared, references to water shall be understood to mean Type IV reagent water at 18 to $27^{\circ} \mathrm{C}$ (specification 0 1193). The method by wich the water is prepared, that is, distillation, ion exchange, reverse ommosis, electrodialysis, or combination thereof, should remain constant throughout testing.

6.3 Sulfuric Acid/Nitric Acid Solution-60/40 reight percent mixture (see section 9.3 sor lnstructions on preparation of this oolution).

\footnotetext{
8nReagent Chemieala, American Chemical Society specifizations," An. Chenical Soc.. Mrhington, DC. For suggestions on the testing of regent not listed by the Aneriean Chemical Society, see angent Chemicals and Standards," by Joseph Rosin. D. Van Nostrand Co.. Inc.. New York, NY, and the "United States Bharmacopeia."
} 


\subsubsection{Empty the ample containez into the center of the nhet.}

8.1.2 Flatten out the ample gestly with aitable atraightedge until It is pread uniformy to depth lest twice the maximum particle diameter.

1.1.3 Remis the ample by difting corner of the aheet and drang It Low cross to the opposit cornex In manner that the

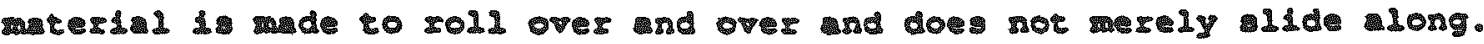
continue operation ith each corner. proceding in clockwise direction. Repert this operation ten tims.

2.1. Iift all four corsers of the sheet towards the center and holding all sour corners together, raise the entire sheet into the li to form pocket for the ample.

\subsubsection{Repeat 8.1.2 to \$latter out the sample.}

8.1.6 With straightedge (such thin-edged yard stick), one at least as long the flattened mound of sample, gently divide the ample into guarters. Make an fiort to avoid using pressure on the straightedge sufficient to cause dange to the particles.

\subsubsection{Discard altersate guarters.}

8.1.8 If Eurthex reduction of ample size is recosary. repeat 0.1.3 through 8.1.7. Use smple date to give 100 of od1d for each extraction. Provide additional samples for determination of solids content (sec section 9.2). If maller samples re used in the test. report this fact.

MoTe - For other scceptable metbod for mixing and ubsapling

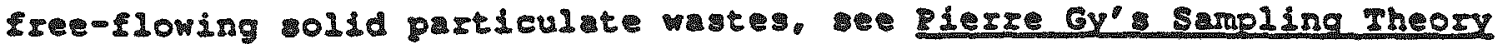
and Sampling Practice, Volume I and II, By I. Pleard, CRC Pres, 2989. The method of ubsampling hould be determined by the physelal properties of the mase, the andytes of interest, and the equipment avadiable.

6.2 For fild-cosed solid waste or castings produced in the laboratory. cut representative section weighing apposinately $100 \mathrm{~g}$ for testing plus amples for deternintion for solids content. Shape the ample so that the leaching solution will cover the material to be 2eached. 
7. 1409

7.1 Obtain representative ample of the wate to be tested using ASTM ampling methods developed for the apecific industry where arible laee ractices D 75 and D 20 , standard rexanology D 653. ard Method D 2234).

7.2 Where no specific methods re vilible. mpling methodology. Lor waterial of ainilax physical form ahll be used.

7.3 The minimum ample aise to be vent to the laboratory hald be det rmined in ccordance pth the seguirement of Method D 2216. section 6.2 .1 (also listed in Figure 2 of this method). and by the zequirement that a ample aize to give $100 \mathrm{~g}$ of anple on dry weight basis is requiged for each extraction. Additiond sample is required sor duplicate determination of solids content (Section 9.2). Inds would be twice the moust specified in Method 2216 and listed in igure 2 .

7. It 1 important that the ample of the wate be zepresentative wth respect to surface area, os viliton in ourince are would directly ffect the leming charactorigics of the sample. wate samples should contin a represertative distribution of particle sizes.

NOTE 3--Information on obtaining representatives amples can also be found in Pierre Gy's Sampling gheory and Sampling practice, volumes I and II, by $F$. Bitard, CRC Rress, 1959.

7.5 Keep sanples in closed contaisers appropriate to the ample type prior to the extraction In order to prevent ample contamination or constituent 10s3. Mhere it i desixed to extract blologically or chenically active anples in their existing state, gtore the samples at

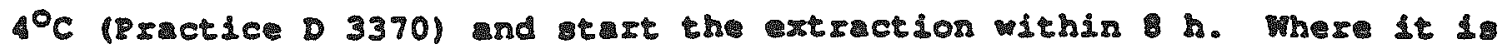
desired to extract uch amples la etro represertative of the results of biological or chemical activities. the amples may be apecifically handled to simulate men activities. Fecord the storage conditions and handing procedures in the report.

\section{Sapolo sepation}

0.1 For free-flowing particulate solid wastes, obtain a ample of the approsimate size reguired in the test by guartering the mple (section 7) received for testing on an impermeable heet of glazed paper, oil cloth, or other lexible naterial hoing composition sustable to the analytes of interest as follows: 
-3 Tor multiphaje stes, mix thoroughy to ensure that a

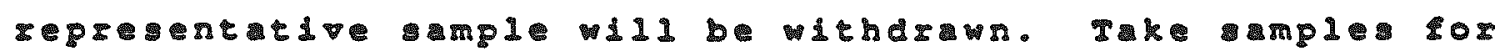
detemination of solids content at the one tine as the test amples.

\section{Irocedure}

9.1 Record the physical description of the arapl to be tested

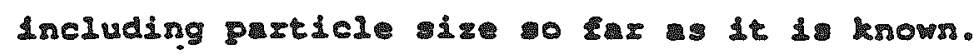

2.2 Solids Content--Determine the old content of two eeparate portions of the ample as folloms:

9.2.1 Dyy to constant weight at $104 \pm 2^{\circ} \mathrm{C}$ two dishes or pans of

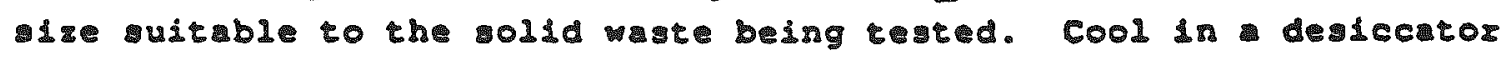
and weigh. Record the values to $\pm 0.1 \mathrm{~g}$.

9.2.2 But an appropiately aized portion of ample of the wate to be tested into each par. Scale the weight used to the physical form of the vaste tested. Use minimum of $100 \mathrm{~g}$ but use larger amples if moze than 10 by weight of the wate is retained on 4.75 men creen (14 sieve) (see Method D 2216 and Figure 2 of thi method). Weigh. Record the mass to $\pm 0.2 \mathrm{~g}$.

9.2.3 Dry 16 to $20 \mathrm{~h}$ at $104 \pm 2{ }^{\circ} \mathrm{C}$. Mecord the temperature and tine of the drying period.

2.2. Cool to soom temperature in desiccator and geweigh. Recosd the mas to $\pm 0.1 \mathrm{~g}$.

9.2.5 Repeat steps 9.2 .3 and 9.2 .4 until constant continezample mase are obtained. Discrd the dried saples coldowing completion of this mep.

2.2.6 Calculate the colids content of the ample from the dat obtained in 9.2 .2 and 9.2 .1 sollows:

$$
5-A / B
$$

were:

A 3 of ample cter drying. g.

- origiral sass of sample, g, and

$s$ - solids content, g/g.

Average the two values obtained. Record the solids content. 


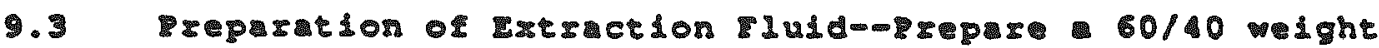
percent mixture of gulfuric acid/nitric acid: Cautiougly mix $60 \mathrm{~g}$ of concentrated ulfuric cid wth 10 g concentrated nitsic acid. Preparation of this mixture hould be performed in laboratory fume bood.

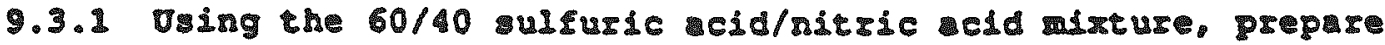
- second solution by diluting 1.0 wi of the $60 / 40$ mixture to 1000 mi using water and a-I volumetric llask.

9.3.2 Using the 2/1000 oution prepared in step 9.3.1. prepare extraction Iluid having the desired prt \pm 0.05 (see 1.2 ) by pipeting a

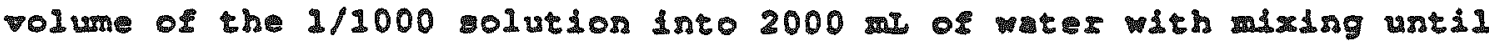
the dealred $\mathrm{pH} \pm 0.05$ is chieved. A zecommended method for preparing the extraction fluid is to ad 2000 an of water to 2-L erlenmeyex Ilask equipped with magnetic stir bar. Flace the exlexmeyer flask on - magnetic stir plate, and add the 1/1000 solution to the 1lask with stixing. Shake the mixture vigoxousy, and once the oolution is static. measure its pH. Continue this process until the desized solution $p H \pm 0.05$ is seached. Record the mount of $1 / 1000$ solution added to 2000 mr of werer to achieve the desired $p$ pH value of the solution. Additional 2-I batches of the ertraction Iluid can be prepared by ming the derermined rolume of 1/1000 solution with $2000 \mathrm{~mL}$ of water. The pH of the extraction Lluid must be within \pm 0.05 of the desired value for use in the extraction procedure. For extracting different wastes reguiring the same extraction fluid ph or performing replicat extractions, multiple batches of extraction fluid can be prepared, measured for correct pH, and if the ph is within \pm 0.05 of the desired value, the batches can be combined in a carboytype container of composition uilable to the rature of the nalyses to be performed. The ptr of the regulting solution in the carboy mugt be measured once agin to verify the correct ph before using the oolution In the extraction proceduse and s sinse polution lstepg 2. Ind 9.7). If the pll 1 not winin the bove specification, the solution ahal be discarded and fresh extraction suid prepared. Kecord the pr value of each batch and of the golution in the carboy przor to its use.

9. Extraction grocedure-Determine the mass of the extretion versel to be used in the extraction procedure to the nerst $0.2 \mathrm{~g}$. Record the mass of the extraction vessel, M1. vse on ertraction vessel per waste throughout the sequence of extractions. 
0.1 .1 Add $100 \mathrm{~g}$ (weighed to $\pm 0.1 \mathrm{~g}$ ) of solid gte on ary weight basis to the extraction vessel. Calculate the anount of ate as recelved to add using the equation:

$$
-\frac{100}{8}
$$

mere:

$$
\begin{aligned}
& s \text { a olids content }(\mathrm{g} / \mathrm{g}) \text { detexmined in section 2.2.6, and } \\
& \text { M mass of mat seceived (nelghed to } \pm 0.1 \text { g) to sdd to }
\end{aligned}
$$

xOTE 5--If mass of aolid wast on dry weight basis other than 100 L used, quation 2.3. and must be modified to reflect the use of ather than 100 . Replace 100 dn these quations wh the mas used.

9.4.2 Add ams in g. Mef. of extraction fluid (see 9.3 ) to the extraction vessel determined using the equations:

$$
M_{\text {SW }}=M-100
$$

mere:

$M_{\text {m }}$ mas of mojsture (g) in the mole added to the extraction
vessel, and

$$
M_{e f}=(20)(200)-M_{\text {sw }}
$$

This wil give oolid-to-1iqgid zato of $1: 20$ in the extraction vessed.

9.4.3 Agit continuously for $18 \pm 0.25$ h at 18 to $27^{\circ} \mathrm{C}$. Record the agitatios time and temperature.

2.4. Oper the extraction vessel. Observe and record any vibible phygical changes in the anple and Leaching oolution. Fecord the phl of the waste/leaching olution lury. 
-.5 Filtration-Transer as much of the mate/lesching solution a posible through large glase funnel to pressure siltration device equipped wth $0.45-\mu$ or $0.3-\mu m$ fidter. Trangfer the mixed 1uryy. Do not decant. Invert the extraction s1tration device and liow the liguid to drain som tho solid remining in ehe extraction veral for 1 minute. It la lmortant to achleve complete transter of fludd from the extraction reasel to the litration device posabie. Bessure sliter the liguid through the fidter using nitrogen gas. Aster the oxtract has pased through the fliter, continu zunning nitrogen gas through the sitzaton device at $30 \mathrm{pg} 1$ Lox 3 minutes. The ilitzate obtained I the extract mentioned In this test method (see 9.6 and 10.8). Determine the mas of the filtrate collected and report it as $\mathrm{M}_{\mathrm{f}}$ for the extraction step. Measure the gh of the extact immediately. remove the amount of filtrate necessary for determination of total dissolved solids content In 9.6. and then preserve the extract in manner consistent with the chemical analyses or biological testing procedures to be performed (Practice D 3370, Section 15 ).

NOTE 6-Analyticad results gna be affected by the type of filtex used. IL $0.8-\mu m$ filtez pore ise la used, the gegulting extract should be digested prior to lemental analysis. The composition of the silter hould 180 be considered. If the I11ter 1 composed of material that may contaninate the extract during filtration, the filter should be washed in the filtration device in maner consistert with the chemical analyses or biological testing procedures to be performed on the extract. For exanple, for elenental analysis of the extract, 11 - fliter composed of borosilicate glass fiber 1 used. It hould be washed in the the filtration device with didute acid oolution and rinsed with appoximately two liters of water prior to ilitration to prevent contamisation.

mote 7-Brefilters can be used only 1 it is absolutely necessisy (filtzate for andysis or tosting can not bo obtained undes prefilter 13 used) due to loss of ample trapped in the pores of the prefilter and the posibility of the prefliter disintegrating dising zinsing.

NOTE - It is recowended that 11 ilitrations be performed in hood. 
2.6 Total Dissolved Solids Content, (TDS)-Andd ech of two 10.0-g anples of the extract to dried, proweighed crucible (meighed to $\pm 0.1 \mathrm{mgl}$. Slace the amples $1 \mathrm{n}$ arying oren at $110^{\circ} \mathrm{C}$ sor $3 \mathrm{~h}$. Record the drying oren temperature and drying elme. Temore the cructble and 2et c001 in desiccator. Rewelgh the crucible and record theis weight to $\pm 0.1 \mathrm{mg}$.

MOTE 9--Only on drylng is performed to 1imit the contact time between the aid and the zinse solution in the extraction vessel prior to the next extraction step (see 2.7, 10 , and 10.7 ).

9.6.1 If the as of solid lost through disolution, Md (sec

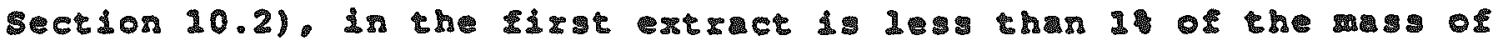
soldd used in the first extraction atep, and is the percent of oolld lost through dssolution dn the second extration step do les than or egual to the percent of solid logt through dissolution in the first extraction step, determination of IDS in the sollowing extract 1 not required, and the user can asume that IDS and $M_{d}$ re gual to 0 for -quations 6 and 9 for extracts three through ter.

9.7 Quantitatively transfer the dang solid from the filter back to the original extraction ressel. including the sidter. Use extraction Lluid. prepared described in section 9.3. Irom preweighed wash bottle to asist in this transter and to zinat the filtration device. No more than $500 \mathrm{~g}$ of cinge solution hould be used. Ose the smaldest anount of sinse solution posible to chieve a thosough trangfer. Using tweerexg or aimilar defice. recover the fiter and rinse the dhering soldd into the crtraction vessol wh rinse extraction sluid from the pre-weighed wash bottle. Do not deave the liter in the extraction vessel. Reweigh the was bottle to determine the mount of rinse solution used in the transfer. Record this value as $M_{R}$. Meigh the extraction vessed following the craster described above and record this vilue M. The extracton vessel my

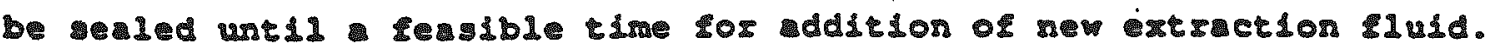
Inis 1. to enable filtration during the next megrence t stamable time during the day. If the luryy s stored for longer than 6 is in the extraction vessel prior to the addition of nem extraction liuid. the dat generated by the analysis of the estracts mould be flotted to check for perturbation of the data curve. 
20

Caleusateros

10.1 Calculate the total dissolved olids content, zos, in silligrams per gram of the liltrate using the following equation:

$$
\text { TDS }=\left(M_{\text {SC }}-M_{C} / / 110 g\right)
$$

where:

Me ms of the crucible and dried solids, ag and

$M_{C}-a_{s}$ of che crucible,

10.2 Calculate the mass of the oold in grams lost through dissolution, Mosing the following equation:

$$
M=\left(\text { IDS) }\left(M_{f}\right)(.001)\right.
$$

were:

$M_{f}=$ ms of filtrate collected in that extraction, g, and

$M_{d}$ mas lost through dissolution.

10.3 Calculate the mass of the solid in grans cosrected for IDS remining for the next extraction atep. Mo uning the following equatios:

$$
M_{3}=M_{3}^{-1}-M_{d}
$$

where:

$$
\begin{gathered}
M^{e-1} \text { mass of the oodid extracted in the current extraction } \\
\text { tep. } 9 .
\end{gathered}
$$

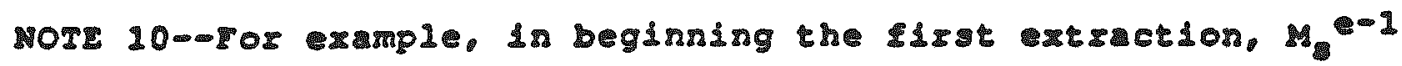
-111 equal $100 \mathrm{~g}$ and to calculate the mas of ooldd remaining for the second extraction tep. $M_{\text {aill equal }} 100$ - $M_{d}$.

10. Calculate the combined mas of the solid and the residual 11quid in the extraction vessed, Mal, using the quation:

$$
M_{S 1}=M_{Y}-M_{1}-M_{R}
$$


10.5 Calculate the mas of liguid adhering to the rolids in the extraction ressel, Mr. using the eguation:

$$
M_{1}=M_{1}-M_{6}
$$

10.6 Calculate the mas in gram of extrectlos fluld to be added to the extraction vessed, Extraction Fluid Mas, (ETM), using the col1owing equation:

$$
\text { EFM }=\left[\left(M_{9}\right)(20)\right]-M_{1}-M_{R}
$$

10.7 Add to the extraction vessel the mount of extraction Eluid, EM, determined sn 10.6 and repert 9.4 .3 through 10.7 a that ten extraction are done in sequence.

NoTe 11--Ihis procedure asumes that the amount of vaste that is crapped in the filtexs fter rinsing is negligible.

10. Anlyze the extact for gpeciflc constiventa or properties of use the extract for biological testing procedures a desired using appropriate ASTM atandard methods. Where no appropriate ASTM methods exist, other methods may be used and mecorded in the report. Whether visible phase separation during the storage of the extrats occurs or not, appopxime mixirg mould be used to ensure tre homogeneity of the ertract prior to their use is such nalyes or testing.

10.9 Compensation for Carry-Orero-For each constituent in each of the extracts generated in the extraction seguence, the contribution to concentration son the residual liguid fron the previous extraction atep, $C_{j}$ can be calculated using the eguation:

$$
c_{j}=\left[M_{1 i} / 20\left(M_{3}{ }^{-1}\right)\right]\left(C_{1}\right]
$$

where:

$$
\begin{aligned}
& c_{i} \text { - concentration of the constituent in the fidtrate from the } \\
& \text { previous extraction step, } \\
& M_{1}-M_{1} \text { rom the previous extraction tep. and } \\
& M_{\text {e-1 }} \text { ms of olid extracted in the curzent extraction atep }
\end{aligned}
$$




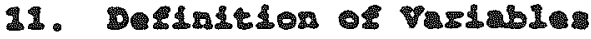

11.1 The following จariables sust be deternined when performing the equentlal bech extaction procedure:

\subsubsection{Solids Content determinaton:}

A - the mass of sample after dxying in the determination of the ooldd content of the wate to be extrated, g.

- the original mas of the ample prior to drying in tho determination of the oolids content of the wast to be extracted, g, and

5 - the olids costert of the st to be extracted, g/g.

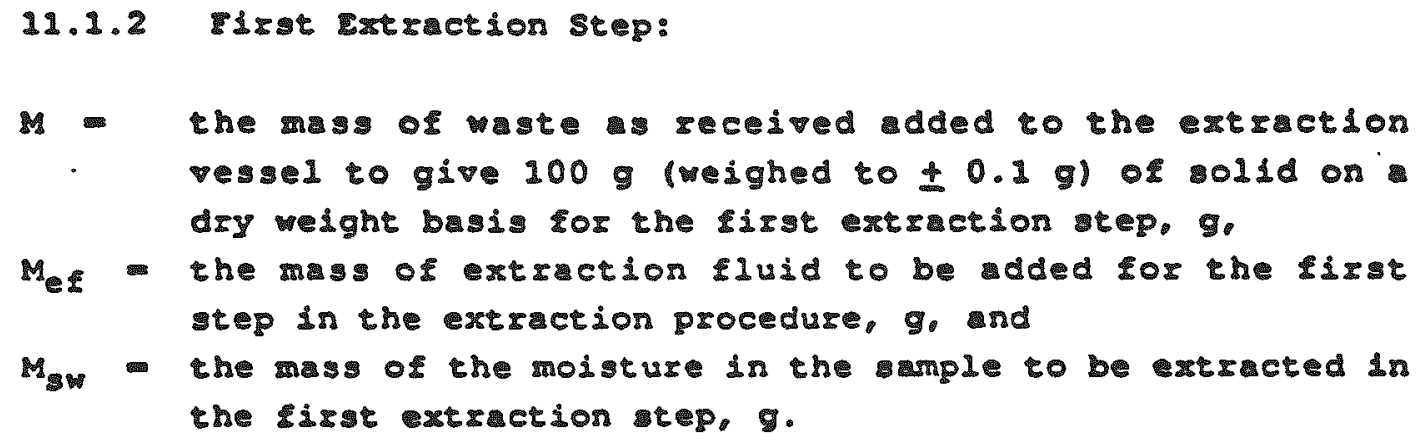

\subsubsection{TDS Determination:}

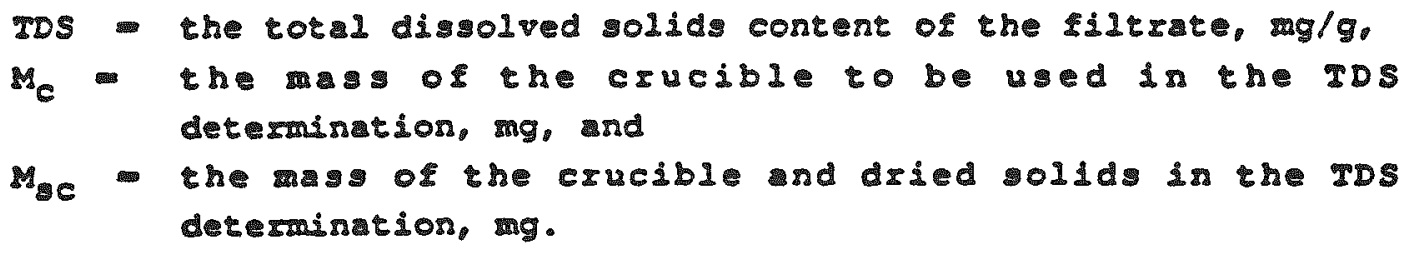

11.1. Ext ction Seguerce:

Md the mas of the solid lost through disolution during extraction, 0

$M_{1}$ - the mas os ofltrate collected in that extraction, g.

$M_{\text {- the }}$ - of the solid remaining for the next extraction tep. 9.

$M_{3} e^{-1}$. thos of the solid extracted in the current extraction tep. g.

Mi - the ss of the empty extraction vessed, g, 


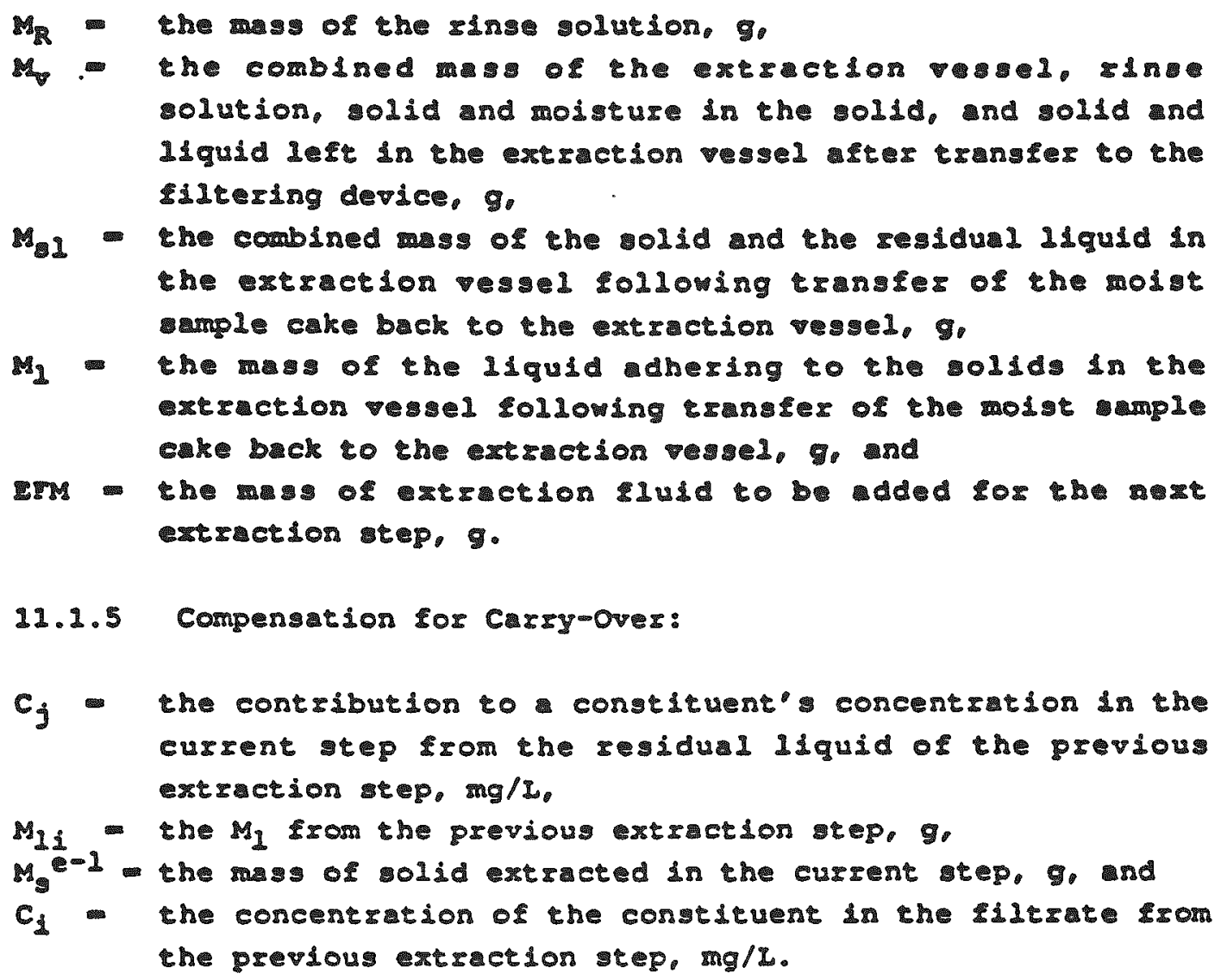

12. Mapost

12.1 Report the sollowing information:

12.1.1 Source of the waste, date of samping, method of sampling, method of ample preservation, sorage conditlong. nd banding procedures,

12.1.2 Description of the wate including physical characteristics and particle alse, di known (9.2).

12.1.3 Solids content (9.2) (see Method D 2216).

12.1. Mass of solid raste extracted if other than $100 \mathrm{~g}(8.2 .8)$.

12.1.5 ph of the extraction fluid used for ach extracton -equence, and as alution for each trangfer,

12.1.6 Iime and temperature used in the determination of solids costent and IOS, 
12.1.7 Agitation terature and tine,

12.1.8 Fiter por wize used and siler composition,

12.1.2 Use of prefilter, grefilter pore size and composition,

i2.1.10 Observitions of chnges in teat material or lesching solution (9.4.4).

12.1.11 storage of the modid with zinge solution in the extraction rosel ston pexiod longer then 6 hours.

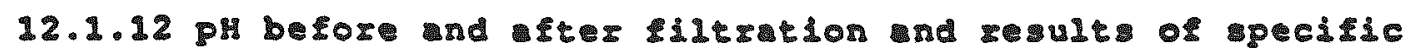
andyses calculated in mpropriate units and corrected sor carsy-orer 16 recessary.

12.1.13 Dates sequential batch extraction started and completed. preservation used for extracts, and dute of andyses.

wore 12-origure 2 present a umary report formt for recording some of the experimental dita and pigure 3 i il derailed laboratory report corm that be belpful in performing the sethod.

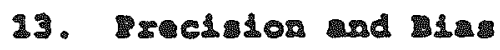

\subsection{Erecision:}

13.1 .1 Dat on the precision of this metrod w11 be generated in an upconing collaborative study of the sethod. Fractice 02777 w11 be used to atatisticaldy evaluate the data.

13.2 Bis-Determisation of the bias of this test method is

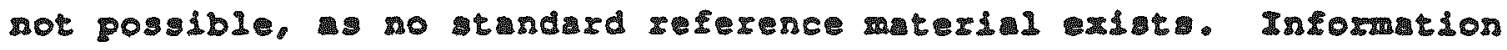
concerning analytical bia wil be obtained rom the andyls of atandard solutions in the upcoming collaborative tudy on the rethod.

14. Kay wosds

14.1 Mate leaching technigue

14.2 Sequential batch extraction

14.3 Extraction siuid

14. Meidic precipitation

14.5 Leaching

14.6 Extract 


\section{(Normandatory Infommtion)}

\section{X1. AGITATION TECRNIQUES AND RATE, MND LIQUID/SOLID RATIO}

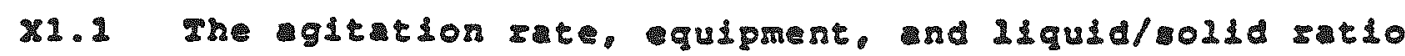
apecifled in the test method, ma sonificanty ingluerce the zesult

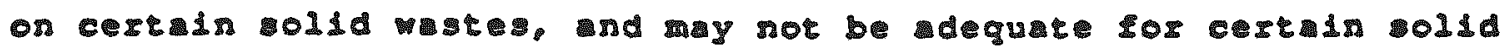
mates, auch monolithic, olidilied, or organie wates.

K1.2 The possible ffects of varying the agtation technigues red rate inelude degree of mixing, zate of selesse of constituents, and particle brasion effects. Ihe precision of the test method ay aro be Irstuenced.

XI.3 The possible effect of vaying the liquid/solid ratio include degree of mixing. rate of release of constituents (and possible concentration effect. depending on valubidtyl. nd particle brasion effect.

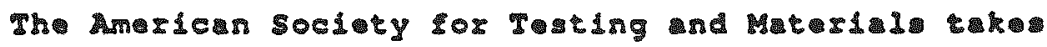

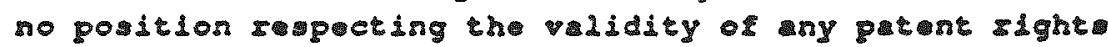
artad in connction with any itom mention in thi

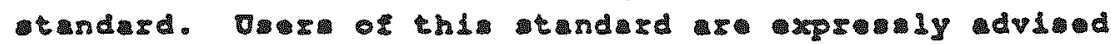
that dotimintion of tho vidity ol ny uch pant

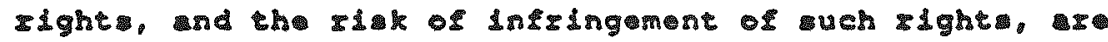

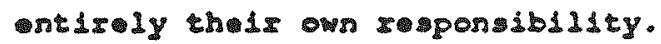

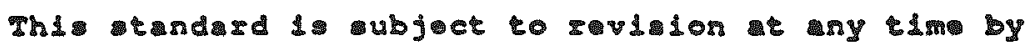

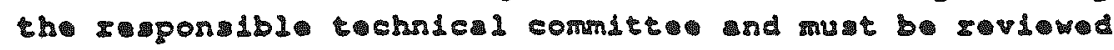

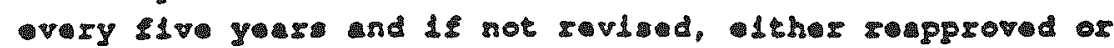

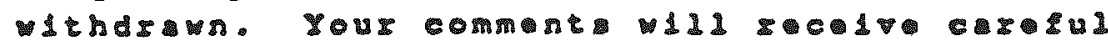

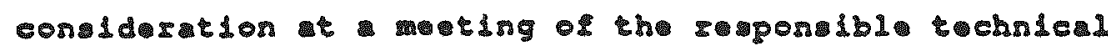
comitto, mich you may atcosd. Is you sol the your

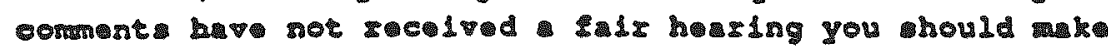

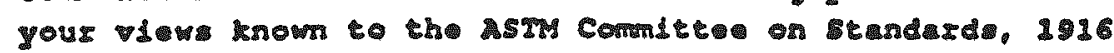

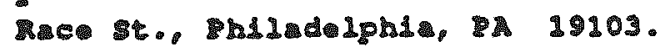



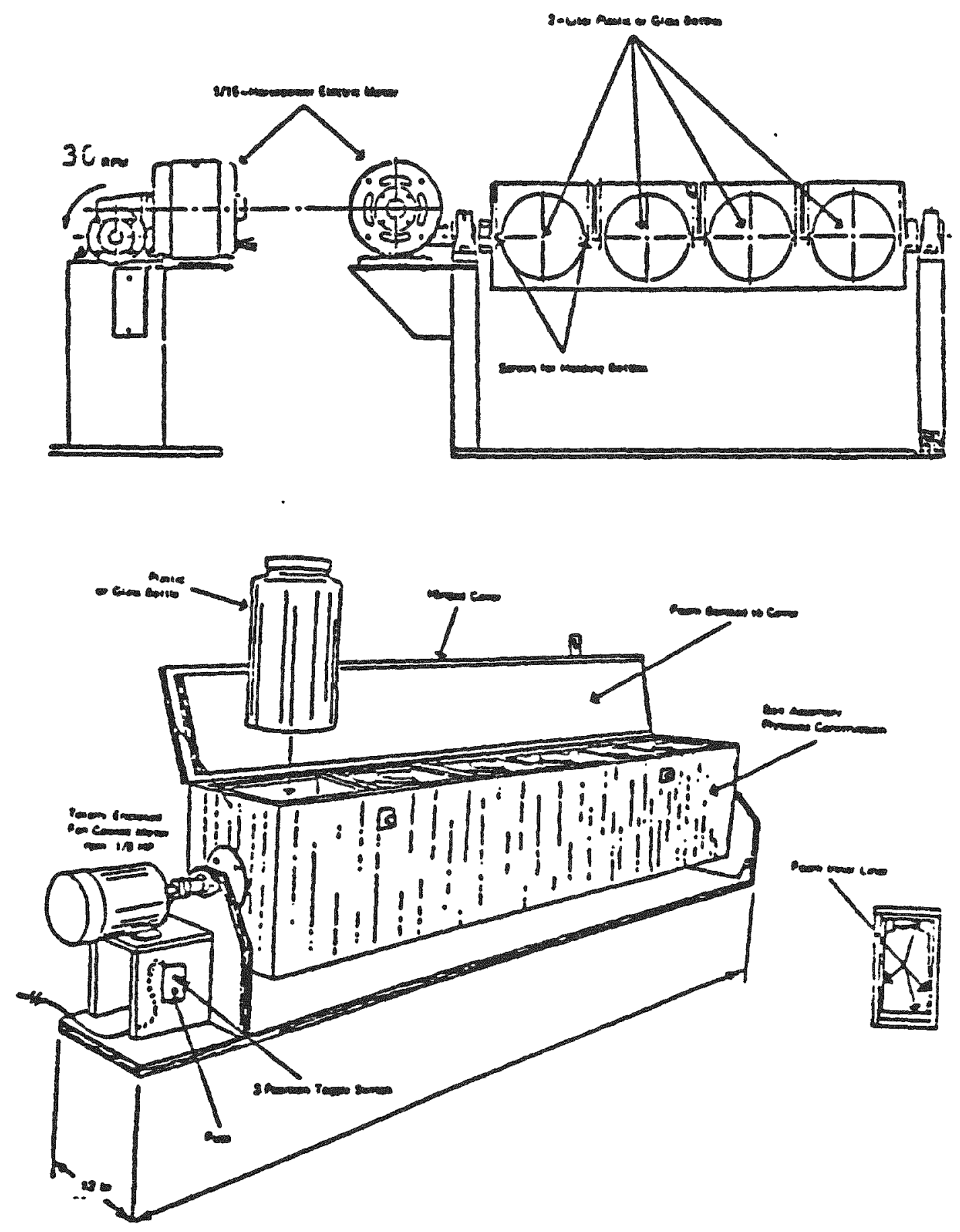

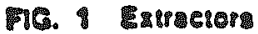




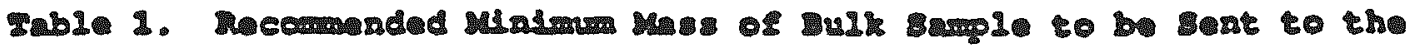

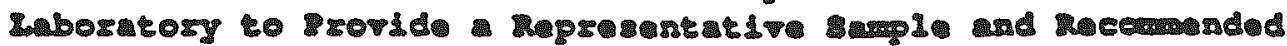

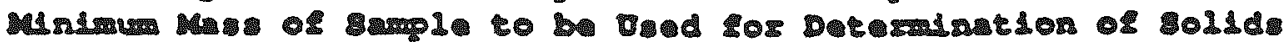
Contant

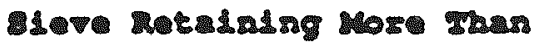

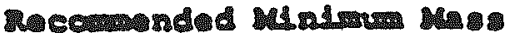

mout 20 of om

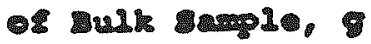

2. (No. 10) S1eve

$100<200$

4.75m (No. S1eve

$300 \div 500$

$19 x$

500201000

38 m

$1500 \$ 3000$

$76 \mathrm{~mm}$

$5000<010,000$

IG. 2 Tab1e 1 


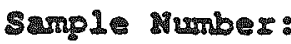

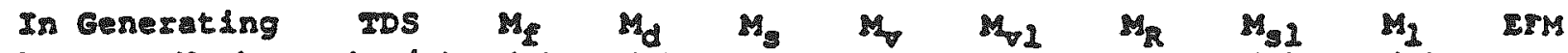
Extract Number (mg/g) (g) $(g)$ (g) $(g)$ (g)

\section{1}

2

3

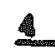

5

6

7

.

9

20

FIG. 3 Sequential Batch Procedure Date Sheet 
10:

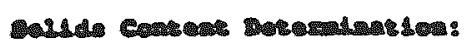

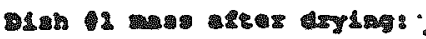

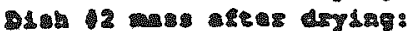

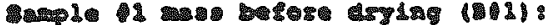

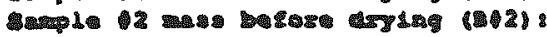

-

25y

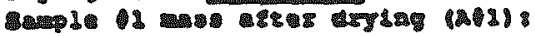

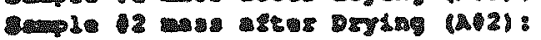

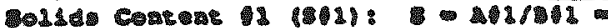

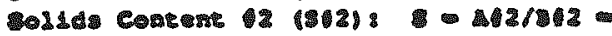

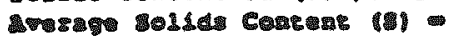

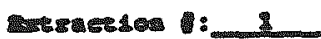

- 2 -

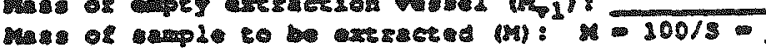

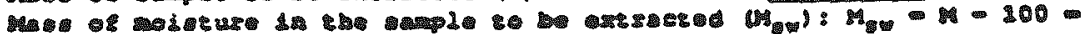

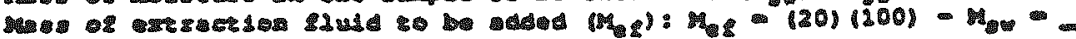

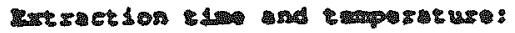

718.

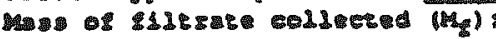

4.

45 - 1 1

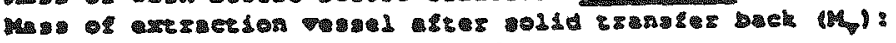

Ma

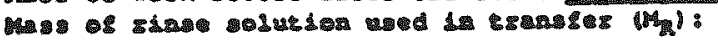

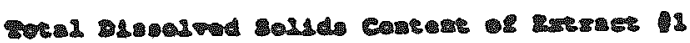

crueibl. 1 (19)

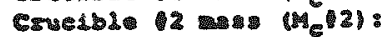

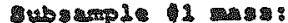

(1) 12 12:

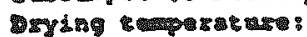

2xy :

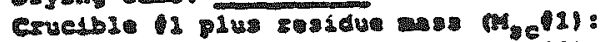

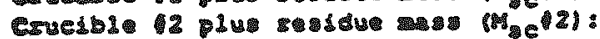

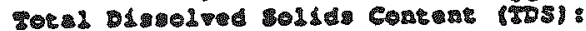

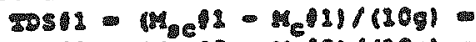

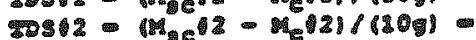

-

-

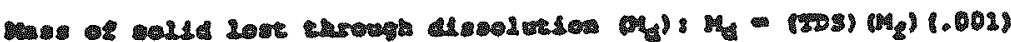

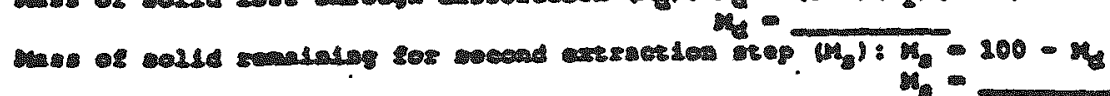

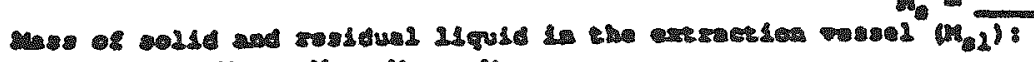

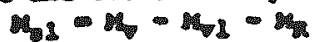

1.

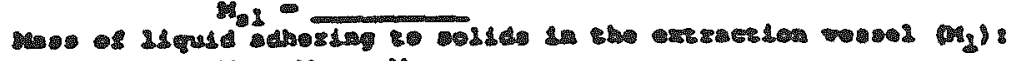

- 1 -

18

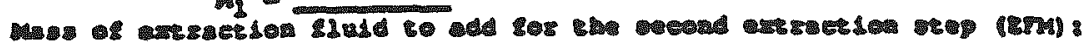
(x) - M $1201-m_{2}$

15. 
16:

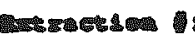
(2)-208

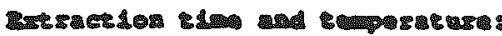

1214:

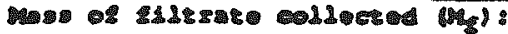

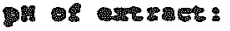

4.

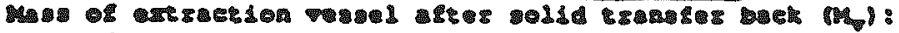

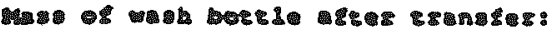

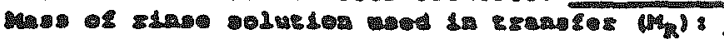

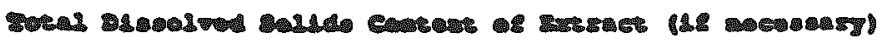

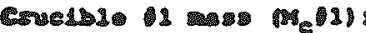

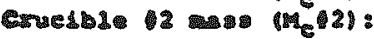

1

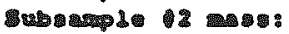

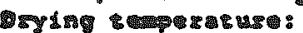

-

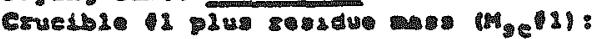

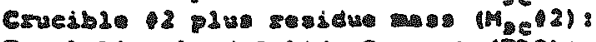

Tor OL

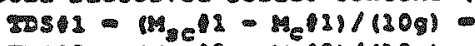

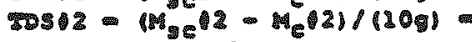

Aพ

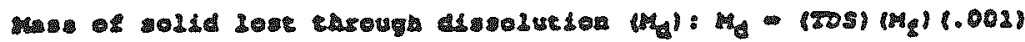

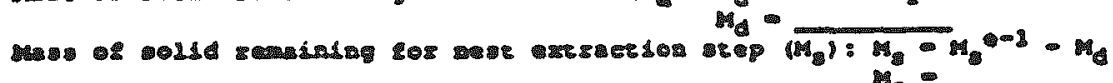

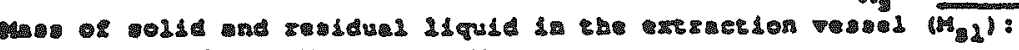

$$
\text { . } 1 \text { - }-1
$$

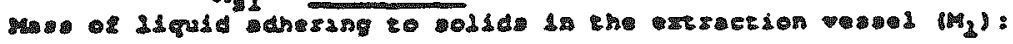

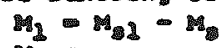

路

M

(1) $(20)-M_{3}-M_{3}$

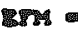

FIG. \& (Cont'd.) Sequential Batch Extraction Laboratory Worksheet 
APPENDIX $B$

Iist of Participating Laboratories in the collaborative study 


\title{
Collaborative study Participants
}

\author{
Listed below are the laboratories that participated in the collaborative \\ study and the name of the contact person. \\ Battelle Pacific Northwest Laboratories \\ Battelle Boulevard \\ P.O. Box 999 \\ Richland, WA 99352 \\ contact: Khris olsen \\ Browning-Ferris Industries, Inc. \\ 5630 Guhn Road \\ Houston, TX 77040 \\ contact: Diana Lasco \\ (teamed with Western Research Institute, \\ performed the analytical portion of the \\ study and extractions of waste $U$ ) \\ Chemical Waste Management, Inc. \\ Technical center \\ $150 \mathrm{~W}$. 137th street \\ Riverdale, IL 60627 \\ contact: Jack Kolopanis \\ CORE Laboratories \\ Analytical Chemistry Division \\ 10703 East Bethany Drive \\ Aurora, CO 80014-2696 \\ contact: Peter Brixius \\ (teamed with Resource Materials Testing, \\ performed the analytical portion of the \\ study) \\ De11 Engineering \\ 245 East Lakewood Blvd. \\ Holland, MI 49424-2066 \\ contact: Robert Zaray \\ Matrix Analytical, Inc. \\ Hopkinton Industrial Park \\ 106 south street \\ Hopkinton, MA 01748 \\ contact: James Todaro \\ Resource Materials Testing \\ 532 Myrtle s.W. \\ Gainsville, GA 30501 \\ contact: Robert Smith \\ Union Carbide corporation \\ Technical center \\ South Charleston, wV 25303 \\ contact: Peter Hannak \\ (teamed with CORE Laboratories, performed \\ the extractions) \\ U.S. Bureau of Mines Spokane Research Center \\ East 915 Montgomery Avenue \\ spokane, WA 99207-2291 \\ contact: Eric zahl \\ Western Research Institute \\ P.O. Box 3395 \\ University station \\ Laramie, WX 82071-3395 \\ contact: Susan Sorini \\ (teamed with Union Carbide Corporation, \\ performed the analytical portion of the \\ study; teamed with Browning-Ferris \\ Industries, performed extractions of \\ waste $M$ )
}


MPPENDIX C

Collaborative study Instructions 


\section{SEQUENTIAL BATCH EXTRACTION METHOD USING DILUTE ACID SOLUTION ASTM INTERLABORATORY STUDY SPECIFICRTIONS}

1. Two different wastes are to be extracted in duplicate using the attached method. These are Waste $U$ and Waste $M$. Duplicate samples of these wastes should be designated as $U-A, U-B$ and $M-A, M-B$. Extracts of the duplicate samples should be designated as $\mathrm{U}-\mathrm{A}-1,-2,-3$, etc, $\mathrm{U}-\mathrm{B}-1,-2$, -3 , etc. and $M-A-1,-2,-3$, etc., $M-B-1,-2,-3$, etc.

2. Waste $U$ is to be extracted using extraction fluid having a pH of $4.30 \pm$ 0.05 , and waste $M$ is to be extracted using extraction fluid having a $\mathrm{pH}$ of $5.00 \pm 0.05$.

3. One blank will be taken through the 10-step extraction procedure. This will consist of extraction fluid, $\mathrm{pH} 4.30 \pm 0.05$, and will be rotated and filtered as a normal sample. Mass of extraction fluid used in the first extraction will be $2000.0 \mathrm{~g}$. The blank extracts should be designated as $B L-1,-2,-3$, etc.

4. A high, medium, and low concentration standard for each extraction fluid $\mathrm{pH}$ has been sent along with the waste samples. These standards are to be analyzed in triplicate using the very same analytical procedures used to analyze the extracts.

5. Eight additional liquid samples have also been sent along with the waste samples and analytical standards. These are labelled M-45, M-45D, M-08, M-08D, U-45, U-45D, U-08, and U-08D. These samples are not to be digested; however, they are to be analyzed using the same analytical method used to analyze the extracts and standards.

6. The analytical parameters for the extracts, standards, and the solutions described in \#5 are listed on their respective data sheets, which are attached.

7. The first extraction step must be started on a Monday and stopped over the weekend. Following filtration and transfer of the solid back to the extraction vessel on Friday, the extraction vessel should be sealed tightly until extraction fluid is added on Monday.

8. For each extraction, the sequential extraction laboratory worksheet that is attached to the method (pages 20 and 21 ) should be completed and sent to the coordinating laboratory along with the other data and information sheets. A copy of this laboratory report is also attached.

9. $100.0 \mathrm{~g}$ of solid on a dry weight basis (section 9.4 .1 of the method) will be used.

10. An 18-hour rotation time will be used. Rotation will be end-over-end at 30 RPM.

11. A pressure filtration device with a $0.45-\mu \mathrm{m}$ nitrocellulose membrane filter will be used for filtering each extraction slurry. prefilters will not be used. 
12. The method of elemental analysis can be determined by the individual participants. Please specify if the extracts were digested prior to analysis and the analytical methods used.

13. Preserve the extracts by acidifying them to $\mathrm{pH}<2$ using a small amount of high purity $\mathrm{BNO}_{3}$.

14. A decision on whether to compensate data for carry-over from one extraction sequence to the next will be made based on a review of the data. If there is substantial carry-over, compensation calculations will be performed by the coordinating laboratory. 
APPENDIX D

Collaborative study questionnaire and Sumuary of Responses 
SEQUENTIAL BATCH EXTRACTION METHOD USING DILUTE ACID SOLUTION ASTM INTERLABORATORY STUDY

INFORMATION FORM

Laboratory:

Date Samples Received:

Date Sequential Batch Extraction started (for each waste):

Date Sequential Batch Extraction Completed (for each waste):

Fxtraction Vesse1 Composition:

Method of Agitation:

Filtration Device:

Comments: 


\section{Participant Responses to the Collaborative Study questionnaire}

A summary of the participants' responses to the questionnaire is given in Table D-1. Laboratory \#1 misplaced their extraction data and did not remember when the samples were received or the dates that the extractions were started or completed. However, they said that they did perform the extractions as specified, starting them on a Monday and stopping the extractions over the weekend (section 7 in collaborative study instructions given in Appendix C). The start and completion dates for the extractions performed by laboratories \#4, \#6, and \#8 show that these laboratories did not follow the specifications of starting their extractions on a Monday and stopping them over the weekend.

Laboratory \#4 added extraction fluid on both Fridays, rotated the slurries for 18 hours, stopped the rotation on saturday, and left the slurries until Monday before filtering. Laboratory \#6 started the extractions on Tuesday and continued them through the weekend. Laboratory \#8 started the extractions on Monday; however, the extraction sequence was stopped on Tuesday and then started again on Wednesday. Laboratory \#8 also stopped the extraction sequence between extractions 7 and 8 for three days, instead of two, over a three-day weekend.

One of the participants did not return their questionnaire, and others did not answer some of the questions on the form or made errors in answering the questions. Participants were contacted by telephone to provide missing information or to clarify the information they had given. None of the participants provided comments on their questionnaires.

A summary of the analytical methods used by the participants in the collaborative study is given in Table D-2. All of the laboratories analyzed their extracts using ICP spectrometry, and none of them, with the exception of laboratories \#7 and \#8, digested the extracts prior to analysis. 
Table D-1. Sumary of the Responses to the Collaborative study Information Form

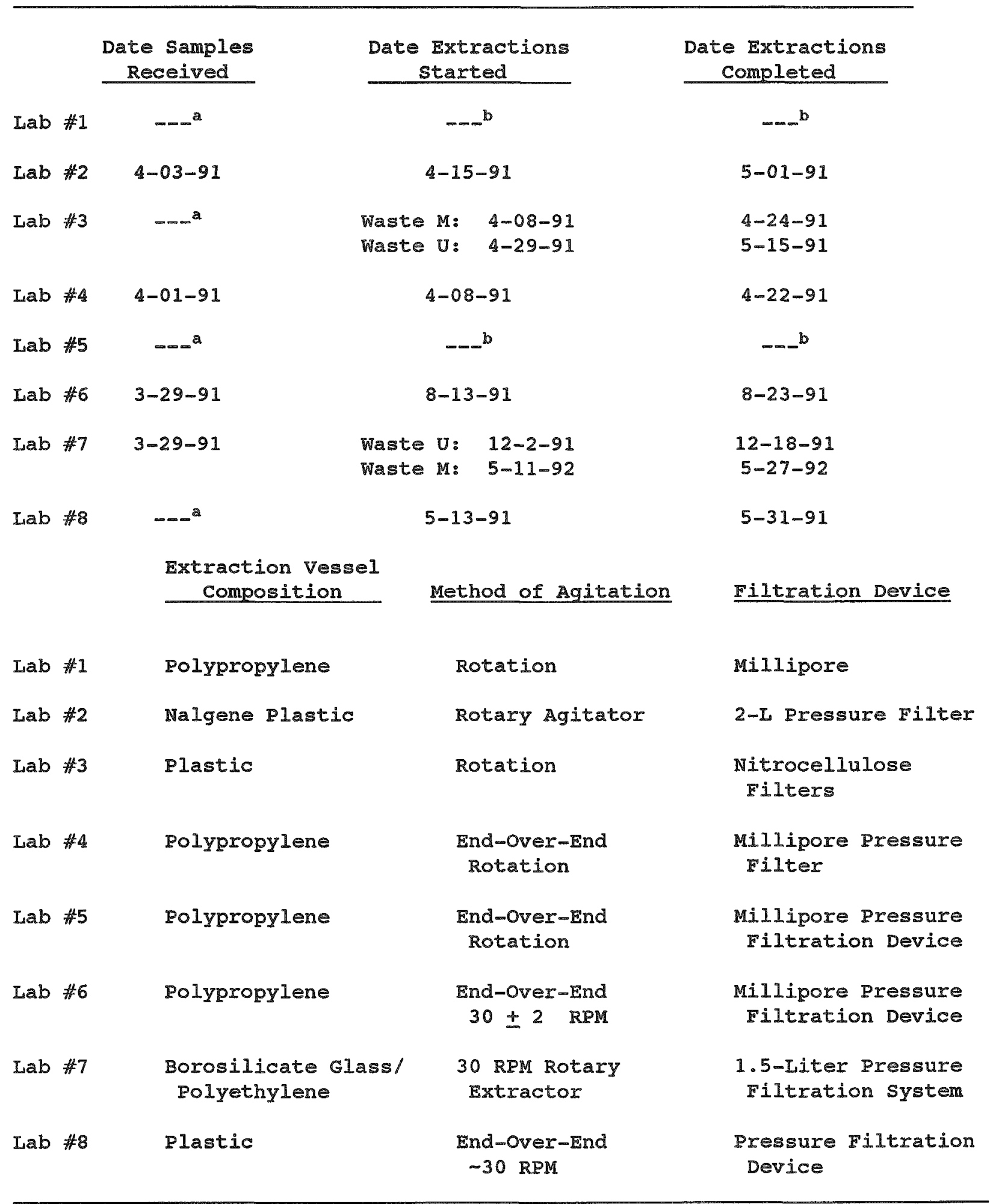

a Not recorded

b Not recorded, but verified by telephone that the extractions were started on a Monday and stopped over the weekend. 
Table D-2. Summary of the Analytical Methods Used in the Collaborative study

\begin{tabular}{|c|c|c|}
\hline Laboratory & Digestion & Analytical Method \\
\hline$\# 1$ & No & $\begin{array}{l}\text { ICP spectrometry: EPA Method } \\
200.7 \text { and sw846 Method } 6010\end{array}$ \\
\hline$\# 2$ & No & $\begin{array}{l}\text { ICP spectrometry: EPA Method } \\
200.7\end{array}$ \\
\hline \#3 & No & $\begin{array}{l}\text { ICP spectrometry (method number } \\
\text { not reported) }\end{array}$ \\
\hline$\# 4$ & No & $\begin{array}{l}\text { ICP spectrometry: EPA Method } \\
200.7\end{array}$ \\
\hline \#5 & No & $\begin{array}{l}\text { ICP spectrometry (method number } \\
\text { not reported) }\end{array}$ \\
\hline \#6 & No & $\begin{array}{l}\text { ICP spectrometry: SW846 Method } \\
6010\end{array}$ \\
\hline \#7 & $\begin{array}{c}\text { Yes } \\
\text { Sw846 Method } 3010\end{array}$ & $\begin{array}{l}\text { ICP spectrometry: } 5 \text { } 846 \text { Method } \\
6010\end{array}$ \\
\hline$\# 8$ & $\begin{array}{c}\text { Yes } \\
\text { sw846 Method } 3020\end{array}$ & $\begin{array}{l}\text { ICP spectrometry: sw846 Method } \\
6010\end{array}$ \\
\hline
\end{tabular}




\section{APPENDIX E}

Collaborative study Data summary sheets 
Extraction Vessel:

Extraction Time and Temperature:

Method of Agitation:

Filter Type and Pore Size:

Filtration Device:

Average solids content:
Polypropylene

Data misplaced

Rotation

$0.45-\mu \mathrm{m}$

Milipore

$\begin{array}{ll}\text { Sample U: } & 0.9867 \mathrm{~g} / \mathrm{g} \\ \text { Sample M: } & 0.9971 \mathrm{~g} / \mathrm{g}\end{array}$

\section{EXTRACPION DATA}

The extraction data were misplaced. The laboratory personnel remember that the TDS value of the second extract for both samples $U$ and $M$ was less than the value required to continue TDs determination on subsequent filtrates. Extraction fluid for the subsequent extractions was added to give a total weight of $2000 \mathrm{~g}$ (extraction fluid plus residual liquid and rinse solution).

\section{PH VALUES}

Waste U: pH Values of Extraction Fluids and Extracts

\begin{tabular}{|c|c|c|c|}
\hline Extract & $\begin{array}{l}\text { pH of Extraction } \\
\text { Fluid Used }\end{array}$ & $\begin{array}{l}\text { pH of Extraction slurry } \\
\text { Before Filtration }\end{array}$ & $\begin{array}{l}\text { pH of Extract } \\
\text { After Filtration }\end{array}$ \\
\hline $\begin{array}{l}U-A-1 \\
U-B-1\end{array}$ & $\begin{array}{l}4 \cdot 3 \\
4 \cdot 3\end{array}$ & $\frac{11}{11} \cdot 5$ & $\begin{array}{l}11.6 \\
11.7\end{array}$ \\
\hline $\begin{array}{l}U-A-2 \\
U-B-2\end{array}$ & $\begin{array}{l}4 \cdot 3 \\
4 \cdot 3\end{array}$ & $\frac{11}{11} \cdot 5$ & $\frac{11}{11} \cdot 4$ \\
\hline $\begin{array}{l}U-A-3 \\
U-B-3\end{array}$ & $\begin{array}{l}4 \cdot 3 \\
4 \cdot 3\end{array}$ & $\begin{array}{l}11 \cdot 3 \\
11.4\end{array}$ & $\frac{11}{11} \cdot \frac{1}{4}$ \\
\hline $\begin{array}{l}U-A-4 \\
U-B-4\end{array}$ & $\begin{array}{l}4 \cdot 3 \\
4 \cdot 3\end{array}$ & $\begin{array}{l}11 \cdot 3 \\
11.4\end{array}$ & $\frac{11}{11} \cdot 4$ \\
\hline $\begin{array}{l}U-A-5 \\
U-B-5\end{array}$ & $\begin{array}{l}4 \cdot 3 \\
4 \cdot 3\end{array}$ & $\begin{array}{l}11 \cdot 3 \\
11 \cdot 3\end{array}$ & $\begin{array}{l}11 \cdot 3 \\
11.3\end{array}$ \\
\hline $\begin{array}{l}U-A-6 \\
U-B-6\end{array}$ & $\begin{array}{l}4 \cdot 3 \\
4 \cdot 3\end{array}$ & $\frac{1}{11} \cdot 2$ & $\begin{array}{l}11 \cdot 3 \\
11 \cdot 3\end{array}$ \\
\hline $\begin{array}{l}U-A-7 \\
U-B-7\end{array}$ & $\begin{array}{l}4 \cdot 4 \\
4.4\end{array}$ & $\frac{11}{11} \cdot 1$ & $\frac{11}{11} \cdot 2$ \\
\hline $\begin{array}{l}U-A-8 \\
U-B-8\end{array}$ & $\begin{array}{l}4 \cdot 3 \\
4 \cdot 3\end{array}$ & 11.0 & $11 \cdot 1$ \\
\hline $\begin{array}{l}U-A-9 \\
U-B-9\end{array}$ & $\begin{array}{l}4.3 \\
4.3\end{array}$ & $\begin{array}{l}10.8 \\
10.9\end{array}$ & $\frac{11}{11} \cdot \frac{1}{1}$ \\
\hline $\begin{array}{l}U-A-10 \\
U-B-10\end{array}$ & $\begin{array}{l}4 \cdot 3 \\
4 \cdot 3\end{array}$ & $\frac{11}{11} \cdot 1$ & $\begin{array}{l}11.0 \\
11.1\end{array}$ \\
\hline
\end{tabular}

a vo value given 
Waste M: $\mathrm{pH}$ Values of Extraction Fluids and Extracts

\begin{tabular}{|c|c|c|c|}
\hline Extract & $\begin{array}{l}\text { pH of Extraction } \\
\text { Fluid Used }\end{array}$ & $\begin{array}{l}\text { pH of Extraction } \\
\text { slurry Before Filtration }\end{array}$ & $\begin{array}{l}\text { oH of Extract } \\
\text { After Filtration }\end{array}$ \\
\hline $\begin{array}{l}M-A-1 \\
M-B-1\end{array}$ & $\begin{array}{l}5.0 \\
5.0\end{array}$ & $\begin{array}{l}6.2 \\
6.1\end{array}$ & $\begin{array}{l}7.2 \\
6.6\end{array}$ \\
\hline $\begin{array}{l}M-A-2 \\
M-B-2\end{array}$ & $\begin{array}{l}5.1 \\
5.1\end{array}$ & $\begin{array}{l}6.3 \\
6.3\end{array}$ & $\begin{array}{l}7.0 \\
6.6\end{array}$ \\
\hline $\begin{array}{l}M-A-3 \\
M-B-3\end{array}$ & 5.1 & $\begin{array}{l}6.9 \\
6.9\end{array}$ & $\begin{array}{l}7.0 \\
6.6\end{array}$ \\
\hline $\begin{array}{l}M-A-4 \\
M-B-4\end{array}$ & $5 \cdot \frac{1}{5 \cdot 1}$ & $\begin{array}{l}7.8 \\
7.8\end{array}$ & $\frac{7.1}{6.8}$ \\
\hline $\begin{array}{l}M-A-5 \\
M-B-5\end{array}$ & $\begin{array}{l}5.1 \\
5.1\end{array}$ & $\begin{array}{l}9.5 \\
9.3\end{array}$ & $\begin{array}{l}9.1 \\
8.8\end{array}$ \\
\hline $\begin{array}{l}M-A-6 \\
M-B-6\end{array}$ & 5.1 & $\begin{array}{l}9.4 \\
9.3\end{array}$ & $\begin{array}{l}8.8 \\
7.9\end{array}$ \\
\hline $\begin{array}{l}M-A-7 \\
M-B-7\end{array}$ & $\begin{array}{l}5 \cdot 1 \\
5.1\end{array}$ & $\begin{array}{l}8.8 \\
8.7\end{array}$ & $\begin{array}{l}7 \cdot 3 \\
8.2\end{array}$ \\
\hline $\begin{array}{l}M-A-8 \\
M-B-8\end{array}$ & $\begin{array}{l}5.1 \\
5.1\end{array}$ & $\begin{array}{l}8.9 \\
9.0\end{array}$ & $\begin{array}{l}8.8 \\
8.9\end{array}$ \\
\hline $\begin{array}{l}M-A-9 \\
M-B-9\end{array}$ & $\begin{array}{l}5.0 \\
5.0\end{array}$ & $\begin{array}{l}8.0 \\
8.1\end{array}$ & $\begin{array}{l}6.4 \\
7.6\end{array}$ \\
\hline $\begin{array}{l}M-A-10 \\
M-B-10\end{array}$ & $\begin{array}{l}5.1 \\
5.1\end{array}$ & $\begin{array}{l}8.3 \\
8.7\end{array}$ & $\begin{array}{l}7.0 \\
7.1\end{array}$ \\
\hline
\end{tabular}

\section{ANATYTICAI INFORMATION}

None of the samples were digested prior to analysis. All samples were analyzed using EPA Method 200.7 and sw846 Method 6010.

\section{DEVIATIONS FROM THE SPECIFIED PROCEDURES}

The sequential extraction laboratory worksheets were misplaced by the laboratory. As a result, calculations could not be checked, and the data could not be compensated for carry-over.

Section 9.3 .2 of the method specifies that the pH of the extraction fluid must be within $t 0.05$ of the desired value for use in the extraction procedure. In performing all of the extractions of Waste M, extraction fluid out of the specified range was used, except for the first and ninth extractions. 


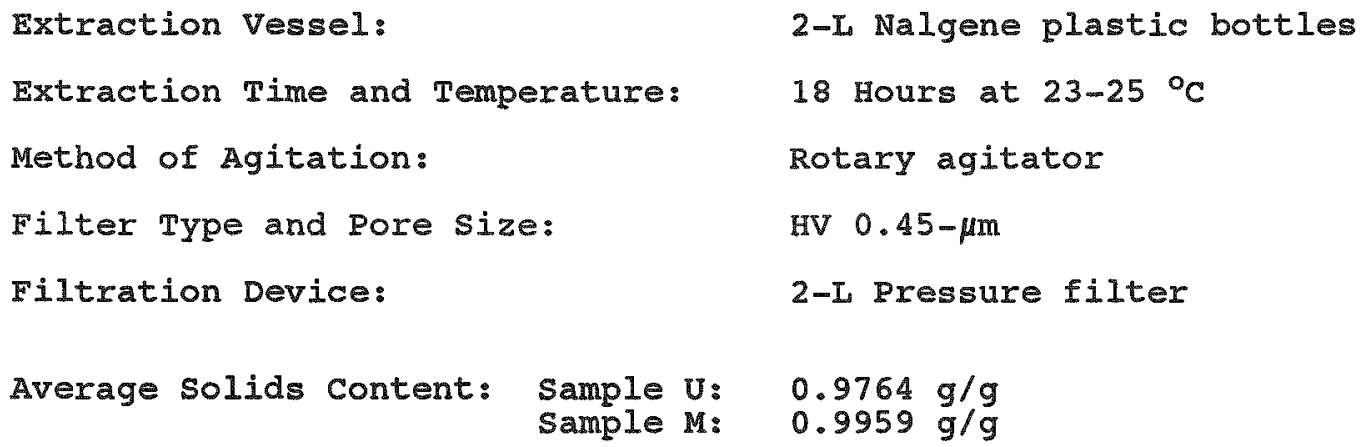

EXTRACTION DATA

WASTE U

\begin{tabular}{cccc}
$\begin{array}{l}\text { In Generating } \\
\text { Extract Number }\end{array}$ & $\begin{array}{c}\text { Average TDS } \\
\text { Content }\end{array}$ & $\begin{array}{c}\text { Mass Lost through } \\
\text { Dissolution, } M_{d} g\end{array}$ & $\begin{array}{c}\text { EFM for Next } \\
\text { Extraction, g }\end{array}$ \\
\hline$U-A-1$ & 0 & 0 & $1,433.3$ \\
$U-B-1$ & 0 & 0 & $1,434.0$ \\
$U-A-2$ & 0 & 0 & $1,434.9$ \\
$U-B-2$ & 0 & 0 & $1,434.1$ \\
$U-A-3$ & 0 & 0 & $1,438.6$ \\
$U-B-3$ & 0 & 0 & $1,430.3$ \\
$U-A-4$ & 0 & 0 & $1,442.5$ \\
$U-B-4$ & 0 & 0 & $1,434.1$ \\
$U-A-5$ & 0 & 0 & $1,443.1$ \\
$U-B-5$ & 0 & 0 & $1,440.7$ \\
$U-A-6$ & 0 & 0 & $1,444.2$ \\
$U-B-6$ & 0 & 0 & $1,444.7$ \\
$U-A-7$ & 0 & 0 & $1,447.9$ \\
$U-B-7$ & 0 & 0 & $1,443.8$ \\
$U-A-8$ & 0 & 0 & $1,456.4$ \\
$U-B-8$ & 0 & 0 & $1,451.2$ \\
$U-A-9$ & 0 & 0 & $1,455.3$ \\
$U-B-9$ & 0 & 0 & $1,452.9$ \\
$U-A-10$ & 0 & 0 & --- \\
$U-B-10$ & 0 & 0 & -1 \\
\hline
\end{tabular}




\section{EXTRACIION DATA}

WASTE M

\begin{tabular}{|c|c|c|c|}
\hline $\begin{array}{l}\text { In Generating } \\
\text { Extract Number }\end{array}$ & $\begin{array}{l}\text { Average TDS } \\
\text { Content }\end{array}$ & $\begin{array}{l}\text { Mass Lost through } \\
\text { Dissolution, } \mathrm{M}_{\mathrm{d}^{\prime}} \mathrm{g}\end{array}$ & $\begin{array}{l}\text { EFM for Next } \\
\text { Extraction, } g\end{array}$ \\
\hline $\begin{array}{l}M-A-1 \\
M-B-1\end{array}$ & $\begin{array}{l}0 \\
0\end{array}$ & $\begin{array}{l}0 \\
0\end{array}$ & $\begin{array}{l}1,480.2 \\
1,481.7\end{array}$ \\
\hline $\begin{array}{l}M-A-2 \\
M-B-2\end{array}$ & $\begin{array}{l}0 \\
0\end{array}$ & $\begin{array}{l}0 \\
0\end{array}$ & $\begin{array}{l}1.479 .8 \\
1.481 .6\end{array}$ \\
\hline $\begin{array}{l}M-A-3 \\
M-B-3\end{array}$ & $\begin{array}{l}0 \\
0\end{array}$ & $\begin{array}{l}0 \\
0\end{array}$ & $\begin{array}{l}1,482 \cdot 6 \\
1,481.4\end{array}$ \\
\hline $\begin{array}{l}M-A-4 \\
M-B-4\end{array}$ & $\begin{array}{l}0 \\
0\end{array}$ & $\begin{array}{l}0 \\
0\end{array}$ & $\begin{array}{l}1.484 .6 \\
1,481.7\end{array}$ \\
\hline $\begin{array}{l}M-\mathbb{A}-5 \\
M-B-5\end{array}$ & $\begin{array}{l}0 \\
0\end{array}$ & $\begin{array}{l}0 \\
0\end{array}$ & $\begin{array}{l}1,492.3 \\
1.490 .9\end{array}$ \\
\hline $\begin{array}{l}M-A-6 \\
M-B-6\end{array}$ & $\begin{array}{l}0 \\
0\end{array}$ & $\begin{array}{l}0 \\
0\end{array}$ & $\begin{array}{l}1,485.8 \\
1.486 .0\end{array}$ \\
\hline $\begin{array}{l}M-A-7 \\
M-B-7\end{array}$ & $\begin{array}{l}0 \\
0\end{array}$ & $\begin{array}{l}0 \\
0\end{array}$ & $\begin{array}{l}1.496 .9 \\
1.487 .0\end{array}$ \\
\hline $\begin{array}{l}M-A-8 \\
M-B-8\end{array}$ & $\begin{array}{l}0 \\
0\end{array}$ & $\begin{array}{l}0 \\
0\end{array}$ & $\begin{array}{l}1,486.2 \\
1,489.1\end{array}$ \\
\hline $\begin{array}{l}M-A-9 \\
M-B-9\end{array}$ & $\begin{array}{l}0 \\
0\end{array}$ & $\begin{array}{l}0 \\
0\end{array}$ & $\begin{array}{l}1,488.1 \\
1,499.2\end{array}$ \\
\hline $\begin{array}{l}M-A-10 \\
M-B-10\end{array}$ & $\begin{array}{l}0 \\
0\end{array}$ & $\begin{array}{l}0 \\
0\end{array}$ & $=-$ \\
\hline
\end{tabular}


SUMMARY SLELT - LABORATORY

\section{PR VATUES}

Waste U: pH Values of Extraction Fluids and Extracts

\begin{tabular}{lccc} 
Extract & $\begin{array}{c}\text { pH of Extraction } \\
\text { Fluid Used }\end{array}$ & $\begin{array}{c}\text { pH of Extraction slurry } \\
\text { Before Filtration }\end{array}$ & $\begin{array}{c}\text { pH of Extract } \\
\text { After Filtration }\end{array}$ \\
\hline U-A-1 & 4.3 & 11.7 & 11.7 \\
U-B-1 & 4.3 & 11.7 & 11.7 \\
U-A-2 & 4.3 & 11.6 & 11.6 \\
U-B-2 & 4.3 & 11.6 & 11.6 \\
U-A-3 & 4.3 & 11.1 & 11.1 \\
U-B-3 & 4.3 & 11.0 & 11.2 \\
U-A-4 & 4.3 & 11.3 & 11.3 \\
U-B-4 & 4.3 & 11.4 & 11.4 \\
U-A-5 & 4.3 & 11.1 & 11.1 \\
$\mathrm{U}-\mathrm{B}-5$ & 4.3 & 11.1 & 11.1 \\
$\mathrm{U}-\mathrm{A}-6$ & 4.3 & 11.1 & 11.1 \\
$\mathrm{U}-\mathrm{B}-6$ & 4.3 & 11.1 & 11.1 \\
$\mathrm{U}-\mathrm{A}-7$ & 4.3 & 10.9 & 11.1 \\
$\mathrm{U}-\mathrm{B}-7$ & 4.3 & 11.1 & 11.2 \\
$\mathrm{U}-\mathrm{A}-8$ & 4.3 & 11.0 & 11.0 \\
$\mathrm{U}-\mathrm{B}-8$ & 4.3 & 11.0 & 11.1 \\
$\mathrm{U}-\mathrm{A}-9$ & 4.3 & 11.1 & 11.1 \\
$\mathrm{U}-\mathrm{B}-9$ & 4.3 & 11.1 & 11.1 \\
$\mathrm{U}-\mathrm{A}-10$ & 4.3 & 11.0 & 11.0 \\
$\mathrm{U}-\mathrm{B}-10$ & 4.3 & 10.9 & 11.0 \\
\hline
\end{tabular}


pH VAIUES

Waste M: pH Values of Extraction Fluids and Extracts

\begin{tabular}{lccc} 
Extract & $\begin{array}{c}\text { pH of Extraction } \\
\text { Fluid Used }\end{array}$ & $\begin{array}{c}\text { pH of Extraction } \\
\text { Slurry }\end{array}$ & $\begin{array}{c}\text { pH of Extract } \\
\text { After Filtration }\end{array}$ \\
\hline M-A-1 & 5.1 & 6.1 & 6.2 \\
M-B-1 & 5.1 & 6.0 & 6.1 \\
M-A-2 & 5.0 & 7.2 & 7.3 \\
M-B-2 & 5.0 & 6.8 & 6.9 \\
M-A-3 & 5.0 & 7.1 & 7.4 \\
M-B-3 & 5.0 & 6.9 & 7.4 \\
M-A-4 & 5.0 & 7.9 & 8.4 \\
M-B-4 & 5.0 & 8.0 & 8.0 \\
M-A-5 & 5.0 & 8.5 & 8.3 \\
M-B-5 & 5.0 & 8.2 & 8.1 \\
M-A-6 & 5.0 & 8.3 & 8.4 \\
M-B-6 & 5.0 & 8.3 & 8.4 \\
M-A-7 & 5.0 & 8.5 & 8.7 \\
M-B-7 & 5.0 & 8.4 & 8.6 \\
M-A-8 & 5.0 & 8.6 & 8.5 \\
M-B-8 & 5.0 & 8.7 & 8.4 \\
M-A-9 & 5.0 & 8.6 & 8.6 \\
M-B-9 & 5.0 & 8.7 & 8.5 \\
M-A-10 & 5.0 & 8.6 & 8.8 \\
M-B-10 & 5.0 & 8.5 & 8.4 \\
& & &
\end{tabular}


ANALYYICAI INEORMATION

None of the samples were digested prior to analysis. All samples were analyzed by ICP using EPA Method 200.7 .

\section{DEVIATIONS FROM THE SPECIFIED PROCEDURES}

1. Section 9.6 of the method specifies that the weighings for TDS determination are to be made to $+0.1 \mathrm{mg}$; however, laboratory \#2 performed their weighings for TDS determination using a balance weighing to only hundredths of a gram, which reduced the sensitivity of their determinations.

2. Section 9.3 .2 of the method specifies that the pH of the extraction fluid must be within \pm 0.05 of the desired value for use in the extraction procedure. - In performing extractions $M-A-1$ and $M-B-1$, extraction fluid having a pH of 5.1 was used, which is out of the specified range of $5 \pm 0.05$.

3. A method blank was to be run with each extraction step; however, a blank was run with only extraction steps 1,9 , and 10 . 


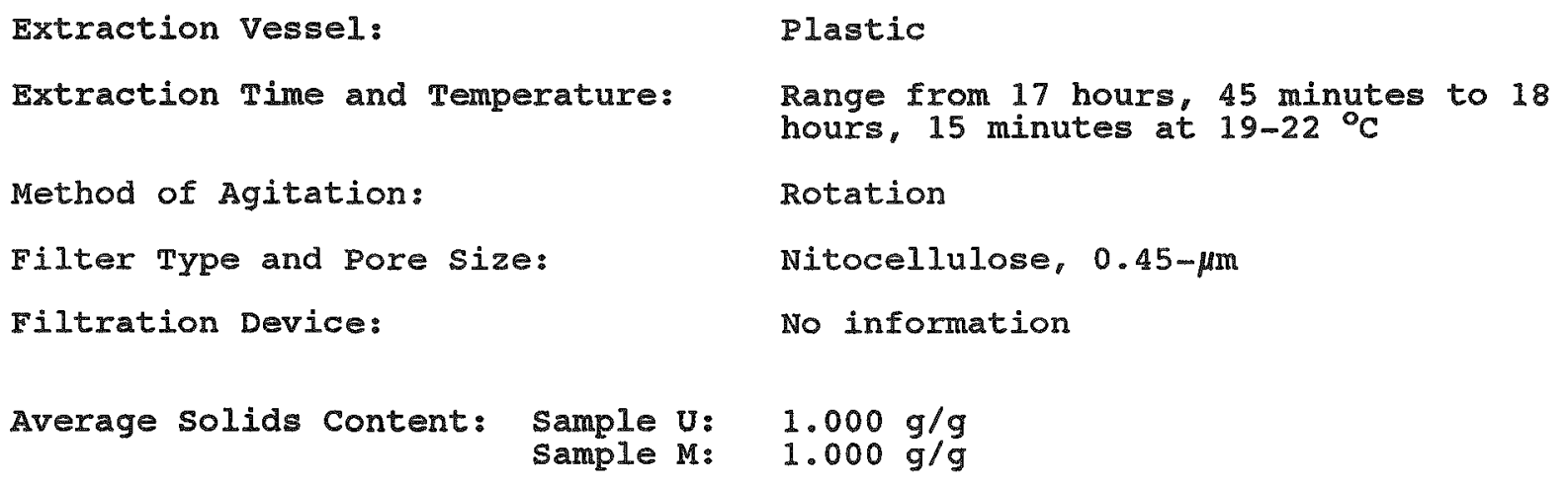

\section{EXTRACTION DATA}

WASTE U

\begin{tabular}{|c|c|c|c|}
\hline $\begin{array}{l}\text { In Generating } \\
\text { Extract Number }\end{array}$ & $\begin{array}{l}\text { Average TDS } \\
\text { Content }\end{array}$ & $\begin{array}{l}\text { Mass Lost through } \\
\text { Dissolution, } \mathrm{M}_{\mathrm{d}^{\prime}} \mathrm{g}\end{array}$ & $\begin{array}{l}\text { EFM for Next } \\
\text { Extraction, } g\end{array}$ \\
\hline $\begin{array}{l}\mathrm{U}-\mathrm{A}-1 \\
\mathrm{U}-\mathrm{B}-1\end{array}$ & $\begin{array}{l}0.001 \\
0.001\end{array}$ & $\begin{array}{l}0.002 \\
0.001\end{array}$ & $\begin{array}{l}1,698.2 \\
1,711.0\end{array}$ \\
\hline $\begin{array}{l}U-A-2 \\
U-B-2\end{array}$ & 0.001 & 0.002 & $\begin{array}{l}1,729.5 \\
1,718.4\end{array}$ \\
\hline $\begin{array}{l}U-A-3 \\
U-B-3\end{array}$ & $\begin{array}{l}0 \\
0\end{array}$ & $\begin{array}{l}0 \\
0\end{array}$ & $\begin{array}{l}1,676.1 \\
1,690.6\end{array}$ \\
\hline $\begin{array}{l}U-A-4 \\
U-B-4\end{array}$ & $\begin{array}{l}0 \\
0\end{array}$ & $\begin{array}{l}0 \\
0\end{array}$ & $\begin{array}{l}1,700.3 \\
1,697.5\end{array}$ \\
\hline $\begin{array}{l}U-A-5 \\
U-B-5\end{array}$ & $\begin{array}{l}0 \\
0\end{array}$ & $\begin{array}{l}0 \\
0\end{array}$ & $\begin{array}{l}1,722.7 \\
1,727.0\end{array}$ \\
\hline $\begin{array}{l}U-A-6 \\
U-B-6\end{array}$ & $\begin{array}{l}0 \\
0\end{array}$ & $\begin{array}{l}0 \\
0\end{array}$ & $\begin{array}{l}1,638.6 \\
1,736.1\end{array}$ \\
\hline $\begin{array}{l}U-A-7 \\
U-B-7\end{array}$ & $\begin{array}{l}0 \\
0\end{array}$ & $\begin{array}{l}0 \\
0\end{array}$ & $\begin{array}{l}1,766.3 \\
1,711.3\end{array}$ \\
\hline $\begin{array}{l}U-A-8 \\
U-B-8\end{array}$ & $\begin{array}{l}0 \\
0\end{array}$ & $\begin{array}{l}0 \\
0\end{array}$ & $\begin{array}{l}1,691.5 \\
1,727.1\end{array}$ \\
\hline $\begin{array}{l}U-A-9 \\
U-B-9\end{array}$ & $\begin{array}{l}0 \\
0\end{array}$ & $\begin{array}{l}0 \\
0\end{array}$ & $\begin{array}{l}1,642 \cdot 5 \\
1,720.7\end{array}$ \\
\hline $\begin{array}{l}U-A-10 \\
U-B-10\end{array}$ & $\begin{array}{l}0 \\
0\end{array}$ & $\begin{array}{l}0 \\
0\end{array}$ & --- \\
\hline
\end{tabular}


SUMMARY SHEET -- LABORATORY \#3

EXTRACTION DATA

WASTE M

\begin{tabular}{|c|c|c|c|}
\hline $\begin{array}{l}\text { In Generating } \\
\text { Extract Number }\end{array}$ & $\begin{array}{l}\text { Average TDS } \\
\text { Content }\end{array}$ & $\begin{array}{l}\text { Mass Lost through } \\
\text { Dissolution, } \mathrm{d}^{\prime} \mathrm{g}\end{array}$ & $\begin{array}{l}\text { EFM for Next } \\
\text { Extraction, } 9\end{array}$ \\
\hline $\begin{array}{l}M-A-1 \\
M-B-1\end{array}$ & $\begin{array}{l}0.0001 \\
0.0004\end{array}$ & $\begin{array}{l}0.0002 \\
0.0008\end{array}$ & ${ }_{-\infty}^{1,451 \cdot 3}$ \\
\hline $\begin{array}{l}M-A-2 \\
M-B-2\end{array}$ & $\begin{array}{l}0.0003 \\
0.0003\end{array}$ & $\begin{array}{l}0.0006 \\
0.0006\end{array}$ & $\begin{array}{l}1,814.9 \\
1,756.1\end{array}$ \\
\hline $\begin{array}{l}M-A-3 \\
M-B-3\end{array}$ & $\begin{array}{l}0.0003 \\
0\end{array}$ & $\begin{array}{l}0.0006 \\
0\end{array}$ & $\begin{array}{l}1.725 .0 \\
1.758 .4\end{array}$ \\
\hline $\begin{array}{l}M-A-4 \\
M-B-4\end{array}$ & $\begin{array}{l}0 \\
0\end{array}$ & $\begin{array}{l}0 \\
0\end{array}$ & $\begin{array}{l}1,754.0 \\
1,684.2\end{array}$ \\
\hline $\begin{array}{l}M-A-5 \\
M-B-5\end{array}$ & $\begin{array}{l}0 \\
0\end{array}$ & $\begin{array}{l}0 \\
0\end{array}$ & $\begin{array}{l}1,740.6 \\
1,738.0\end{array}$ \\
\hline $\begin{array}{l}M-A-6 \\
M-B-6\end{array}$ & $\begin{array}{l}0 \\
0\end{array}$ & $\begin{array}{l}0 \\
0\end{array}$ & $\begin{array}{l}1.717 .3 \\
1.559 .0\end{array}$ \\
\hline $\begin{array}{l}M-A-7 \\
M-B-7\end{array}$ & $\begin{array}{l}0 \\
0\end{array}$ & $\begin{array}{l}0 \\
0\end{array}$ & $\begin{array}{l}1,698.8 \\
1,680.3\end{array}$ \\
\hline $\begin{array}{l}M-A-8 \\
M-B-8\end{array}$ & $\begin{array}{l}0 \\
0\end{array}$ & $\begin{array}{l}0 \\
0\end{array}$ & $\begin{array}{l}1,663.9 \\
1,644.7\end{array}$ \\
\hline $\begin{array}{l}M-A-9 \\
M-B-9\end{array}$ & $\begin{array}{l}0 \\
0\end{array}$ & $\begin{array}{l}0 \\
0\end{array}$ & $\begin{array}{l}1,678.9 \\
1,696.1\end{array}$ \\
\hline $\begin{array}{l}M-A-10 \\
M-B-10\end{array}$ & $\begin{array}{l}0 \\
0\end{array}$ & $\begin{array}{l}0 \\
0\end{array}$ & $\begin{array}{l}--- \\
---\end{array}$ \\
\hline
\end{tabular}

$a_{\text {No }}$ value given 
SUMMARY SHEET -- LABORATORY 3

pH VAIUES

Waste U: pH Values of Extraction Fluids and Extracts

\begin{tabular}{|c|c|c|c|}
\hline Extract & $\begin{array}{l}\text { pH of Extraction } \\
\text { Fluid Used }\end{array}$ & $\begin{array}{l}\text { pH of Extraction slurry } \\
\text { Before Filtration }\end{array}$ & $\begin{array}{l}\mathrm{pH} \text { of Extract } \\
\text { After Filtration }\end{array}$ \\
\hline $\begin{array}{l}U-A-1 \\
U-B-1\end{array}$ & $\begin{array}{l}4 \cdot 3 \\
4.3\end{array}$ & $\begin{array}{l}11.6 \\
11.6\end{array}$ & $\begin{array}{l}11.5 \\
11.6\end{array}$ \\
\hline $\begin{array}{l}U-A-2 \\
U-B-2\end{array}$ & $\begin{array}{l}4.3 \\
4.3\end{array}$ & $\begin{array}{l}11 \cdot 3 \\
11 \cdot 4\end{array}$ & $\begin{array}{l}11 \cdot 3 \\
11.3\end{array}$ \\
\hline $\begin{array}{l}U-A-3 \\
U-B-3\end{array}$ & $\begin{array}{l}4 \cdot 3 \\
4 \cdot 3\end{array}$ & $\begin{array}{l}11 \cdot 2 \\
11.3\end{array}$ & $\begin{array}{l}11.2 \\
11.2\end{array}$ \\
\hline $\begin{array}{l}U-A-4 \\
U-B-4\end{array}$ & $\begin{array}{l}4.3 \\
4.3\end{array}$ & $\begin{array}{l}11.1 \\
11.2\end{array}$ & $\begin{array}{l}11.2 \\
11.1\end{array}$ \\
\hline $\begin{array}{l}U-A-5 \\
U-B-5\end{array}$ & $\begin{array}{l}4.3 \\
4.3\end{array}$ & $\begin{array}{l}11.1 \\
11.2\end{array}$ & $\begin{array}{l}11.0 \\
11.1\end{array}$ \\
\hline $\begin{array}{l}U-A-6 \\
U-B-6\end{array}$ & $\begin{array}{l}4 \cdot 3 \\
4 \cdot 3\end{array}$ & $\begin{array}{l}11.1 \\
11.2\end{array}$ & $\begin{array}{l}11.1 \\
11.2\end{array}$ \\
\hline $\begin{array}{l}U-A-7 \\
U-B-7\end{array}$ & $\begin{array}{l}4 \cdot 3 \\
4 \cdot 3\end{array}$ & $\begin{array}{l}10.9 \\
10.9\end{array}$ & $\begin{array}{l}10.9 \\
10.9\end{array}$ \\
\hline $\begin{array}{l}\mathrm{U}-\mathrm{A}-8 \\
\mathrm{U}-\mathrm{B}-8\end{array}$ & $\begin{array}{l}4.3 \\
4.3\end{array}$ & $\begin{array}{l}10.8 \\
10.6\end{array}$ & $\begin{array}{l}11.0 \\
10.7\end{array}$ \\
\hline $\begin{array}{l}U-A-9 \\
U-B-9\end{array}$ & $\begin{array}{l}4.3 \\
4.3\end{array}$ & $\begin{array}{l}10.9 \\
10.9\end{array}$ & $\begin{array}{l}10.9 \\
10.9\end{array}$ \\
\hline $\begin{array}{l}U-A-10 \\
U-B-10\end{array}$ & $\begin{array}{l}4.3 \\
4.3\end{array}$ & $\begin{array}{l}10.9 \\
10.8\end{array}$ & $\begin{array}{l}10.9 \\
10.8\end{array}$ \\
\hline
\end{tabular}


SURMARY SHEET -- LABORATORY \#3

\section{pH VALUES}

Waste M: $\mathrm{pH}$ Values of Extraction Fluids and Extracts

\begin{tabular}{|c|c|c|c|}
\hline Extract & $\begin{array}{l}\text { pH of Extraction } \\
\text { Fluid Used }\end{array}$ & $\begin{array}{l}\text { pH of Extraction } \\
\text { slurry Before Filtration }\end{array}$ & $\begin{array}{l}\mathrm{pH} \text { of Extract } \\
\text { After Filtration }\end{array}$ \\
\hline $\begin{array}{l}M-A-1 \\
M-B-1\end{array}$ & $\begin{array}{l}5.0 \\
5.0\end{array}$ & $\begin{array}{l}5.9 \\
6.1\end{array}$ & $\overline{5.9}$ \\
\hline $\begin{array}{l}\mathrm{M}-\mathrm{A}-2 \\
\mathrm{M}-\mathrm{B}-2\end{array}$ & $\begin{array}{l}5.0 \\
5.0\end{array}$ & $\begin{array}{l}6.1 \\
5.8\end{array}$ & $\begin{array}{l}5.9 \\
5.7\end{array}$ \\
\hline $\begin{array}{l}M-A-3 \\
M-B-3\end{array}$ & $\begin{array}{l}5.0 \\
5.0\end{array}$ & $\begin{array}{l}5.8 \\
5.8\end{array}$ & $\begin{array}{l}6.0 \\
5.8\end{array}$ \\
\hline $\begin{array}{l}M-A-4 \\
M-B-4\end{array}$ & $\begin{array}{l}5.0 \\
5.0\end{array}$ & $\begin{array}{l}7.0 \\
7.0\end{array}$ & $\begin{array}{l}7.3 \\
7.2\end{array}$ \\
\hline $\begin{array}{l}M-A-5 \\
M-B-5\end{array}$ & $\begin{array}{l}5.0 \\
5.0\end{array}$ & $\begin{array}{l}7.7 \\
7.6\end{array}$ & $\begin{array}{l}7.7 \\
7.4\end{array}$ \\
\hline $\begin{array}{l}M-A-6 \\
M-B-6\end{array}$ & $\begin{array}{l}5.0 \\
5.0\end{array}$ & $\begin{array}{l}7.8 \\
7.7\end{array}$ & $\begin{array}{l}8.0 \\
8.0\end{array}$ \\
\hline $\begin{array}{l}\mathrm{M}-\mathrm{A}-7 \\
\mathrm{M}-\mathrm{B}-7\end{array}$ & $\begin{array}{l}5.0 \\
5.0\end{array}$ & $\begin{array}{l}7.8 \\
7.8\end{array}$ & $\begin{array}{l}8.2 \\
8.2\end{array}$ \\
\hline $\begin{array}{l}\mathrm{M}-\mathrm{A}-8 \\
\mathrm{M}-\mathrm{B}-8\end{array}$ & $\begin{array}{l}5.0 \\
5.0\end{array}$ & $\begin{array}{l}8.0 \\
8.0\end{array}$ & $\begin{array}{l}8.2 \\
8.2\end{array}$ \\
\hline $\begin{array}{l}M-A-9 \\
M-B-9\end{array}$ & $\begin{array}{l}5.0 \\
5.0\end{array}$ & $\begin{array}{l}7.9 \\
7.9\end{array}$ & $\begin{array}{l}8.3 \\
8.4\end{array}$ \\
\hline $\begin{array}{l}M-A-10 \\
M-B-10\end{array}$ & $\begin{array}{l}5.0 \\
5.0\end{array}$ & $\begin{array}{l}8.2 \\
8.4\end{array}$ & $\begin{array}{l}8.3 \\
8.4\end{array}$ \\
\hline
\end{tabular}

${ }^{a}$ No value given 
SUMMARY SHEET - - LABORATORY 3

MNALYTICAI INFORMATION

None of the samples were digested prior to analysis. All samples were analyzed using ICP.

\section{DEVIATIONS FROM THE SPECIFIED PROCEDURES}

1. There were many errors in calculating EFM. In some cases, these resulted in the addition of an incorrect amount of extraction fluid. The following are the errors in calculating EFM, TDS, and $M_{d}$ that were detected in reviewing this laboratory's data.

a. In calculating EFM values for $U-A-6$ through $U-A-9$, it appears that the $M$ value for the U-B samples was used.

Amount Added

$\begin{array}{ll}U-A-6 & 1,638.69 \\ U-A-7 & 1,766.39 \\ U-A-8 & 1,691.59 \\ U-A-9 & 1,642.59\end{array}$

Correct Amount

$$
\begin{aligned}
& 1,641.6 \mathrm{~g} \\
& 1,769.3 \mathrm{~g} \\
& 1,694.5 \mathrm{~g} \\
& 1,645.5 \mathrm{~g}
\end{aligned}
$$

b. For $M-A-1$ and $M-A-2$, there were errors in calculating $\operatorname{TDS}$ and $M_{\text {. }}$ (These have no effect on the EFM values. However, since the incorrect values resulted in TDS determination in step 3 for sample M-A, the TDS determination should have continued throughout the rest of the extraction procedure.)

c. Errors in calculating EFM for sample M:

Amount Added

$$
\begin{aligned}
& M-A-1 \\
& M-A-4 \\
& M-A-6 \\
& M-A-8 \\
& M-B-2 \\
& M-B-6
\end{aligned}
$$

$$
\begin{aligned}
& 1,451.3 \mathrm{~g} \\
& 1,754.09 \\
& 1,717.39 \\
& 1,663.99 \\
& 1,756.19 \\
& 1,559.09
\end{aligned}
$$

Correct Amount

$1,541.7 \mathrm{~g}$

$1,704.09$

$1,722.0 \mathrm{~g}$

$1,669.99$

$1,755.09$

$1,648.99$

2. For sample $M-A-3$, some spillage while pouring the solution into the pressure filter was noted.

3. Section 9.6 of the method specifies that the weighings for TDS determination are to be made to $+0.1 \mathrm{mg}$; however, laboratory \#3 performed their weighings for TDS determination using a balance weighing to one miligram, which reduced the sensitivity of their determinations. 


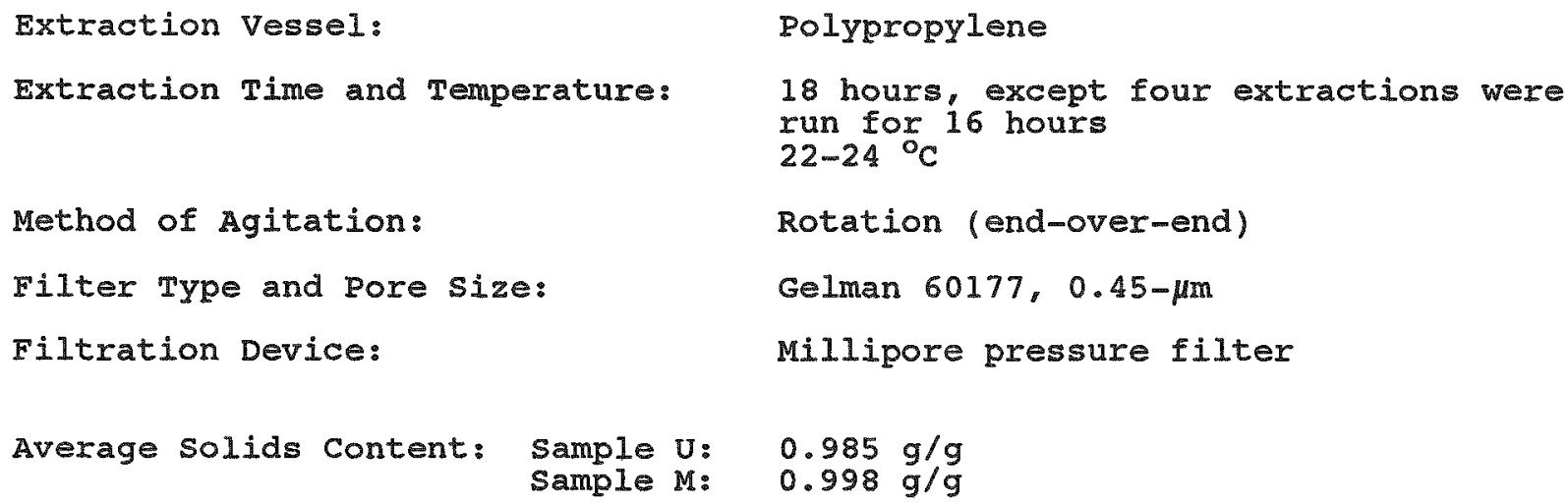

EXTRACTION DATA

WASTE U

\begin{tabular}{|c|c|c|c|}
\hline $\begin{array}{l}\text { In Generating } \\
\text { Extract Number }\end{array}$ & $\begin{array}{l}\text { Average TDS } \\
\text { Content }\end{array}$ & $\begin{array}{l}\text { Mass Lost through } \\
\text { Dissolution, Ma, } g\end{array}$ & $\begin{array}{l}\text { EFM for Next } \\
\text { Extraction, } 9\end{array}$ \\
\hline $\begin{array}{l}U-A-1 \\
U-B-1\end{array}$ & $\begin{array}{l}0.0025 \\
0.0011\end{array}$ & $\begin{array}{l}0.0050 \\
0.002\end{array}$ & $\begin{array}{l}1.848 .6 \\
1.851 .8\end{array}$ \\
\hline $\begin{array}{l}U-A-2 \\
U-B-2\end{array}$ & $\begin{array}{l}0.0005 \\
0.0005\end{array}$ & $\begin{array}{l}0.001 \\
0.0009\end{array}$ & $\begin{array}{l}1,736.8 \\
1,904.3\end{array}$ \\
\hline $\begin{array}{l}U-A-3 \\
U-B-3\end{array}$ & $\begin{array}{l}0.00025 \\
0.00045\end{array}$ & $\begin{array}{l}0.0004 \\
0.0008\end{array}$ & $\begin{array}{l}1,846.3 \\
1,918.2\end{array}$ \\
\hline $\begin{array}{l}U-A-4 \\
U-B-4\end{array}$ & $\begin{array}{l}0.0003 \\
0.0005\end{array}$ & $\begin{array}{l}0.0005 \\
0.0009\end{array}$ & $\begin{array}{l}1,856.5 \\
1,747.2\end{array}$ \\
\hline $\begin{array}{l}U-A-5 \\
U-B-5\end{array}$ & $\begin{array}{l}0.00007 \\
0.0002\end{array}$ & $\begin{array}{l}0.001 \\
0.0003\end{array}$ & $\begin{array}{l}1,883.6 \\
1,948.6\end{array}$ \\
\hline $\begin{array}{l}U-A-6 \\
U-B-6\end{array}$ & $\begin{array}{l}0 \\
0\end{array}$ & $\begin{array}{l}0 \\
0\end{array}$ & $\begin{array}{l}1.935 .4 \\
1.916 .6\end{array}$ \\
\hline $\begin{array}{l}U-A-7 \\
U-B-7\end{array}$ & $\begin{array}{l}0 \\
0\end{array}$ & $\begin{array}{l}0 \\
0\end{array}$ & $\begin{array}{l}1.924 .5 \\
1,910.9\end{array}$ \\
\hline $\begin{array}{l}U-A-8 \\
U-B-8\end{array}$ & $\begin{array}{l}0 \\
0\end{array}$ & $\begin{array}{l}0 \\
0\end{array}$ & $\begin{array}{l}1,937.1 \\
1,930.1\end{array}$ \\
\hline $\begin{array}{l}U-A-9 \\
U-B-9\end{array}$ & $\begin{array}{l}0 \\
0\end{array}$ & $\begin{array}{l}0 \\
0\end{array}$ & $\begin{array}{l}1,932.7 \\
1,910.9\end{array}$ \\
\hline $\begin{array}{l}U-A-10 \\
U-B-10\end{array}$ & $\begin{array}{l}0 \\
0\end{array}$ & $\begin{array}{l}0 \\
0\end{array}$ & --- \\
\hline
\end{tabular}


SUMMARY SHEET -- LABORATORY \#4

\section{EXTRACTION DATA}

WASTE M

\begin{tabular}{|c|c|c|c|}
\hline $\begin{array}{l}\text { In Generating } \\
\text { Extract Number }\end{array}$ & $\begin{array}{l}\text { Average TDS } \\
\text { Content }\end{array}$ & $\begin{array}{l}\text { Mass Lost through } \\
\text { Dissolution, } \mathrm{M}^{\prime} g\end{array}$ & $\begin{array}{l}\text { EFM for Next } \\
\text { Extraction, } g\end{array}$ \\
\hline $\begin{array}{l}M-A-1 \\
M-B-1\end{array}$ & $\begin{array}{l}0.00037 \\
0.00039\end{array}$ & $\begin{array}{l}0.0007 \\
0.0007\end{array}$ & $\begin{array}{l}1,847.7 \\
1,824.4\end{array}$ \\
\hline $\begin{array}{l}M-A-2 \\
M-B-2\end{array}$ & $\begin{array}{l}0.00003 \\
0.00008\end{array}$ & $\begin{array}{l}0.00005 \\
0.0014\end{array}$ & $\begin{array}{l}1.865 .3 \\
1.838 .4\end{array}$ \\
\hline $\begin{array}{l}M-A-3 \\
M-B-3\end{array}$ & $\begin{array}{l}0.00003 \\
0\end{array}$ & $\begin{array}{l}0.00005 \\
0\end{array}$ & $\begin{array}{l}1,834.0 \\
1,926.8\end{array}$ \\
\hline $\begin{array}{l}M-A-4 \\
M-B-4\end{array}$ & $\begin{array}{l}0.0001 \\
0.00043\end{array}$ & $\begin{array}{l}0.002 \\
0.0008\end{array}$ & $\begin{array}{l}1,909.7 \\
1,932.9\end{array}$ \\
\hline $\begin{array}{l}M-A-5 \\
M-B-5\end{array}$ & $\begin{array}{l}0.00005 \\
0\end{array}$ & $\begin{array}{l}0.00009 \\
0\end{array}$ & $\begin{array}{l}1,935.0 \\
1,915.9\end{array}$ \\
\hline $\begin{array}{l}M-A-6 \\
M-B-6\end{array}$ & $\begin{array}{l}0 \\
0\end{array}$ & $\begin{array}{l}0 \\
0\end{array}$ & $\begin{array}{l}1,892.2 \\
1,877.7\end{array}$ \\
\hline $\begin{array}{l}M-A-7 \\
M-B-7\end{array}$ & $\begin{array}{l}0 \\
0\end{array}$ & $\begin{array}{l}0 \\
0\end{array}$ & $\begin{array}{l}1,897.5 \\
1,901.0\end{array}$ \\
\hline $\begin{array}{l}M-A-8 \\
M-B-8\end{array}$ & $\begin{array}{l}0 \\
0\end{array}$ & $\begin{array}{l}0 \\
0\end{array}$ & $\begin{array}{l}1,899.8 \\
1,884.0\end{array}$ \\
\hline $\begin{array}{l}M-A-9 \\
M-B-9\end{array}$ & $\begin{array}{l}0 \\
0\end{array}$ & $\begin{array}{l}0 \\
0\end{array}$ & $\begin{array}{l}1,917.6 \\
1,892.4\end{array}$ \\
\hline $\begin{array}{l}M-A-10 \\
M-B-10\end{array}$ & $\begin{array}{l}0 \\
0\end{array}$ & $\begin{array}{l}0 \\
0\end{array}$ & $\begin{array}{l}-- \\
---\end{array}$ \\
\hline
\end{tabular}


SUMMARY SHEET -- LABORATORY \#4

\section{PH VALUES}

Waste U: $\mathrm{pH}$ Values of Extraction Fluids and Extracts

\begin{tabular}{lccc} 
Extract & $\begin{array}{c}\text { pH of Extraction } \\
\text { Fluid Used }\end{array}$ & $\begin{array}{c}\text { pH of Extraction slurry } \\
\text { Before }\end{array}$ & $\begin{array}{c}\text { pH of Extract } \\
\text { After Filtration }\end{array}$ \\
\hline U-A-1 & 4.3 & 10.7 & 11.6 \\
U-B-1 & 4.3 & 10.6 & 11.6 \\
U-A-2 & 4.3 & 10.6 & 11.5 \\
U-B-2 & 4.3 & 10.9 & 11.2 \\
U-A-3 & 4.3 & 10.5 & 11.6 \\
U-B-3 & 4.3 & 10.6 & 11.6 \\
U-A-4 & 4.3 & 10.8 & 11.7 \\
U-B-4 & 4.3 & 10.5 & 11.8 \\
U-A-5 & 4.3 & 10.6 & 11.6 \\
U-B-5 & 4.3 & 10.7 & 11.6 \\
U-A-6 & 4.3 & 10.7 & 11.6 \\
U-B-6 & 4.3 & 10.8 & 11.6 \\
U-A-7 & 4.3 & 10.8 & 10.6 \\
U-B-7 & 4.3 & 10.5 & 11.6 \\
U-A-8 & 4.3 & 10.8 & 11.6 \\
U-B-8 & 4.3 & 10.7 & 11.5 \\
U-A-9 & 4.3 & 10.8 & 11.7 \\
U-B-9 & 4.3 & 10.9 & 11.6 \\
U-A-10 & 4.3 & 10.7 & 11.6 \\
U-B-10 & 4.3 & 10.5 & 11.6 \\
& & &
\end{tabular}




\section{PI VALUES}

Waste M: $\mathrm{pH}$ Values of Extraction Fluids and Extracts

\begin{tabular}{|c|c|c|c|}
\hline Extract & $\begin{array}{l}\text { pH of Extraction } \\
\text { Fluid Used }\end{array}$ & $\begin{array}{l}\text { pH of Extraction } \\
\text { Slurry Before Filtration }\end{array}$ & $\begin{array}{l}\text { pH of Extract } \\
\text { After Filtration }\end{array}$ \\
\hline $\begin{array}{l}M-A-1 \\
M-B-1\end{array}$ & $\begin{array}{l}5.0 \\
5.0\end{array}$ & $\begin{array}{l}5.5 \\
5.2\end{array}$ & $\begin{array}{l}5.6 \\
5.7\end{array}$ \\
\hline $\begin{array}{l}M-A-2 \\
M-B-2\end{array}$ & $\begin{array}{l}5.0 \\
5.0\end{array}$ & $\begin{array}{l}5.6 \\
5.4\end{array}$ & $\begin{array}{l}5.7 \\
5.9\end{array}$ \\
\hline $\begin{array}{l}M-A-3 \\
M-B-3\end{array}$ & $\begin{array}{l}5.0 \\
5.0\end{array}$ & $\begin{array}{l}5.1 \\
5.3\end{array}$ & $\begin{array}{l}5.8 \\
5.7\end{array}$ \\
\hline $\begin{array}{l}M-A-4 \\
M-B-4\end{array}$ & $\begin{array}{l}5.0 \\
5.0\end{array}$ & $\begin{array}{l}5.4 \\
5.5\end{array}$ & $\begin{array}{l}5.8 \\
5.9\end{array}$ \\
\hline $\begin{array}{l}M-A-5 \\
M-B-5\end{array}$ & $\begin{array}{l}5.0 \\
5.0\end{array}$ & $\begin{array}{l}5.2 \\
5.3\end{array}$ & $\begin{array}{l}5.6 \\
5.8\end{array}$ \\
\hline $\begin{array}{l}M-A-6 \\
M-B-6\end{array}$ & $\begin{array}{l}5.0 \\
5.0\end{array}$ & $\begin{array}{l}5.4 \\
5.4\end{array}$ & $\begin{array}{l}5.7 \\
5.8\end{array}$ \\
\hline $\begin{array}{l}\mathrm{M}-\mathrm{A}-7 \\
\mathrm{M}-\mathrm{B}-7\end{array}$ & $\begin{array}{l}5.0 \\
5.0\end{array}$ & $\begin{array}{l}5.1 \\
5.3\end{array}$ & $\begin{array}{l}5.7 \\
5.7\end{array}$ \\
\hline $\begin{array}{l}M-A-8 \\
M-B-8\end{array}$ & $\begin{array}{l}5.0 \\
5.0\end{array}$ & $\begin{array}{l}5.3 \\
5.2\end{array}$ & $\begin{array}{l}5.8 \\
5.7\end{array}$ \\
\hline $\begin{array}{l}M-A-9 \\
M-B-9\end{array}$ & $\begin{array}{l}5.0 \\
5.0\end{array}$ & $\begin{array}{l}5 \cdot 3 \\
5.3\end{array}$ & $\begin{array}{l}5.8 \\
5.7\end{array}$ \\
\hline $\begin{array}{l}M-A-10 \\
M-B-10\end{array}$ & $\begin{array}{l}5.0 \\
5.0\end{array}$ & $\begin{array}{l}5.5 \\
5.3\end{array}$ & $\begin{array}{l}5.8 \\
5.7\end{array}$ \\
\hline
\end{tabular}


None of the samples were digested prior to analysis. All samples were analyzed by ICP using EPA Method 200.7 .

\section{DEVIATIONS FROM THE SPECIFIED PROCEDURES}

1. Item \#7 1isted in the interlaboratory study specifications states that the first extraction step must be started on a Monday and the procedure stopped over the weekend. It also specifies that following filtration and transfer of the solid back to the extraction vessel on Friday, the extraction vessel should be sealed tightly until extraction fluid is added on Monday. Despite these instructions, laboratory \#4 added extraction fluid on both Fridays, rotated the slurries for 18 hours, stopped the rotation on saturday, and left the slurries until Monday before filtering.

2. Extractions $M-A-1, M-A-3, M-B-1$, and $U-B-1$ had an extraction time of 16 hours, rather than $18 \pm 0.25$ hours as specified in the procedure.

3. There were some errors in calculating EFM. These resulted in the addition of incorrect amounts of extraction fluid. The errors in calculating EFM that were detected are as follows:

Amount Added

$$
\begin{array}{ll}
U-A-2 & 1,736.89 \\
U-B-4 & 1,747.29 \\
M-A-5 & 1,935.09
\end{array}
$$

Correct Amount

$$
\begin{aligned}
& 1,900.2 \mathrm{~g} \\
& 1,908.3 \mathrm{~g} \\
& 1,928.8 \mathrm{~g}
\end{aligned}
$$

4. Also, in many cases there were errors in calculating TDS, and as a result, $M_{0}$. These do not affect the EFM values. The errors resulted in the ros determinations being continued past the second extraction, when they should have been stopped. The lab stopped TDs determinations after the fifth extraction, which is not specified in the procedure. Errors in calculating TDS were identified for extractions $U-A-1, U-A-2$, $U-A-5, M-A-3, M-A-4$, and $M-B-2$. 
Extraction Vessel:
Extraction Time and Temperature:

Method of Agitation:

Filter Type and Pore size:

Filtration Device:

Average solids content:
Polypropylene

$18+0.25$ hours, except the first extraction for U-A, U-B, M-A, and M-B went for 23 hours $20.3-26.7{ }^{\circ} \mathrm{C}$

Rotation (end-over-end)

Millipore HAWP $142,0.45-\mu \mathrm{m}$

Milipore pressure filtration device

$0.983 \mathrm{~g} / \mathrm{g}$

$0.998 \mathrm{~g} / \mathrm{g}$

\section{EXTRACTION DATA}

WASTE U

In Generating Extract Number
EFM for Next Extraction, $g$

\begin{tabular}{|c|c|}
\hline $\begin{array}{l}U-\bar{A}-1 \\
U-B-1\end{array}$ & $\begin{array}{l}2,048.0 \\
2,041.0\end{array}$ \\
\hline $\begin{array}{l}U-A-2 \\
U-B-2\end{array}$ & $\begin{array}{l}2,041.0 \\
2,036.0\end{array}$ \\
\hline $\begin{array}{l}U-A-3 \\
U-B-3\end{array}$ & $\begin{array}{l}2,030.0 \\
2,036.0\end{array}$ \\
\hline $\begin{array}{l}U-A-4 \\
U-B-4\end{array}$ & $\begin{array}{l}2,018.0 \\
2,029.0\end{array}$ \\
\hline $\begin{array}{l}U-A-5 \\
U-B-5\end{array}$ & $\begin{array}{l}2,023.0 \\
2,028.0\end{array}$ \\
\hline $\begin{array}{l}U-A-6 \\
U-B-6\end{array}$ & $\begin{array}{l}2,020.0 \\
2,025.0\end{array}$ \\
\hline $\begin{array}{l}U-A-7 \\
U-B-7\end{array}$ & $\begin{array}{l}2,033.0 \\
2,030.0\end{array}$ \\
\hline $\begin{array}{l}U-A-8 \\
U-B-8\end{array}$ & $\begin{array}{l}2,027.0 \\
2,030.0\end{array}$ \\
\hline $\begin{array}{l}U-A-9 \\
U-B-9\end{array}$ & $\begin{array}{l}2,025.0 \\
2,029.0\end{array}$ \\
\hline $\begin{array}{l}U-A-10 \\
U-B-10\end{array}$ & -- \\
\hline
\end{tabular}


SUMMARY SHEET -- LABORATORY \#5

EXTRACTION DATA

WASTE M

In Generating

Extract Number
EFM for Next

Extraction, $g$
$M-A-1$

$\mathrm{M}-\mathrm{B}-1$

$M-A-2$

$\mathrm{M}-\mathrm{B}-2$

M-A -3

$M-B-3$

$M-A-4$

$M-B-4$

$M-A-5$

$M-B-5$

$M-A-6$

$\mathrm{M}-\mathrm{B}-6$

$\mathrm{M}-\mathrm{A}-7$

$M-B-7$

$M-A-8$

M-B-8

M-A-9

$M-B-9$

M-A-10

M-B- 10
$2,041.0$

$2,024.0$

$2,043.0$

$2,054.0$

$2,030.0$

$2,045.0$

$2,017.0$

$2,035.0$

$2,032.0$

$2,026.0$

$2,026.0$

$2,029.0$

$2,056.0$

$2,029.0$

$2,028.0$

2,031 .0

$2,061.0$

$2,032.0$

$--\infty$ 


\section{PH VALUES}

Waste U: pH Values of Extraction Fluids and Extracts

\begin{tabular}{|c|c|c|c|}
\hline Extract & $\begin{array}{l}\text { pH of Extraction } \\
\text { Fluid Used }\end{array}$ & $\begin{array}{l}\text { pH of Extraction slurry } \\
\text { Before Filtration }\end{array}$ & $\begin{array}{l}\text { pH of Extract } \\
\text { After Filtration }\end{array}$ \\
\hline $\begin{array}{l}U-A-1 \\
U-B-1\end{array}$ & $\begin{array}{l}4 \cdot 3 \\
4.3\end{array}$ & $\begin{array}{l}11.8 \\
11.8\end{array}$ & $--^{2}$ \\
\hline $\begin{array}{l}U-A-2 \\
U-B-2\end{array}$ & $\begin{array}{l}4.3 \\
4.3\end{array}$ & $\begin{array}{l}11 \cdot 5 \\
11 \cdot 5\end{array}$ & $\begin{array}{l}-\infty \\
--\infty\end{array}$ \\
\hline $\begin{array}{l}U-A-3 \\
U-B-3\end{array}$ & $\begin{array}{l}4.3 \\
4.3\end{array}$ & $\begin{array}{l}11 \cdot 5 \\
11 \cdot 5\end{array}$ & --- \\
\hline $\begin{array}{l}U-A-4 \\
U-B-4\end{array}$ & $\begin{array}{l}4 \cdot 3 \\
4 \cdot 3\end{array}$ & $\begin{array}{l}11 \cdot 4 \\
11.4\end{array}$ & $\begin{array}{l}-\infty- \\
---\end{array}$ \\
\hline $\begin{array}{l}U-A-5 \\
U-B-5\end{array}$ & $\begin{array}{l}4 \cdot 3 \\
4 \cdot 3\end{array}$ & $\begin{array}{l}11 \cdot 3 \\
11.3\end{array}$ & $-\infty$ \\
\hline $\begin{array}{l}U-A-6 \\
U-B-6\end{array}$ & $\begin{array}{l}4.3 \\
4.3\end{array}$ & $\begin{array}{l}11 \cdot 5 \\
11 \cdot 4\end{array}$ & $-\infty-$ \\
\hline $\begin{array}{l}U-A-7 \\
U-B-7\end{array}$ & $\begin{array}{l}4.3 \\
4.3\end{array}$ & $\begin{array}{l}11.4 \\
11.4\end{array}$ & --- \\
\hline $\begin{array}{l}U-A-8 \\
U-B-8\end{array}$ & $\begin{array}{l}4.3 \\
4.3\end{array}$ & $\begin{array}{l}11.4 \\
11.4\end{array}$ & $-\infty$ \\
\hline $\begin{array}{l}U-A-9 \\
U-B-9\end{array}$ & $\begin{array}{l}4.3 \\
4.3\end{array}$ & $\begin{array}{l}11.4 \\
11.4\end{array}$ & -- \\
\hline $\begin{array}{l}U-A-10 \\
U-B-10\end{array}$ & $\begin{array}{l}4.3 \\
4.3\end{array}$ & $\begin{array}{l}11.2 \\
11.2\end{array}$ & $\begin{array}{l}11.2 \\
11.2\end{array}$ \\
\hline
\end{tabular}

a No value given 
PH VAIUSS

Waste M: pH Values of Extraction Fluids and Extracts

\begin{tabular}{lccc} 
Extract & $\begin{array}{c}\text { pH of Extraction } \\
\text { Fluid Used }\end{array}$ & $\begin{array}{c}\text { pH of Extraction } \\
\text { Slurry }\end{array}$ & $\begin{array}{c}\text { pH of Extract } \\
\text { After }\end{array}$ \\
\hline Filtration
\end{tabular}

${ }^{a}$ No value given 
SUMAMRY SHEET -- LABORATORY \#5

\section{ANALYTICAL INEORMATION}

A11 samples were analyzed, undigested, by ICAP.

\section{DEVIATIONS FROM THE SPECIFIED PROCEDURES}

1. Laboratory \#5 did not perform the extraction procedure as specified. TDS determinations were not performed. The mass of solid lost through dissolution $\left(M_{d}\right)$ and the mass of liguid adhering to the solid in the extraction vessel (M) were not calculated. The equation for calculating EFM is $\left(M_{s}\right)(20)-M_{1}-M_{0}$. However, laboratory \#5 had no value for $M_{1}$, and incorrectly added $M_{R}$ to $\left(M_{s}\right)(20)$, rather than subtracting the $M_{R}$ value as specified by the equation. $M_{s}$ was assumed to equal 100 for all of the extractions. 


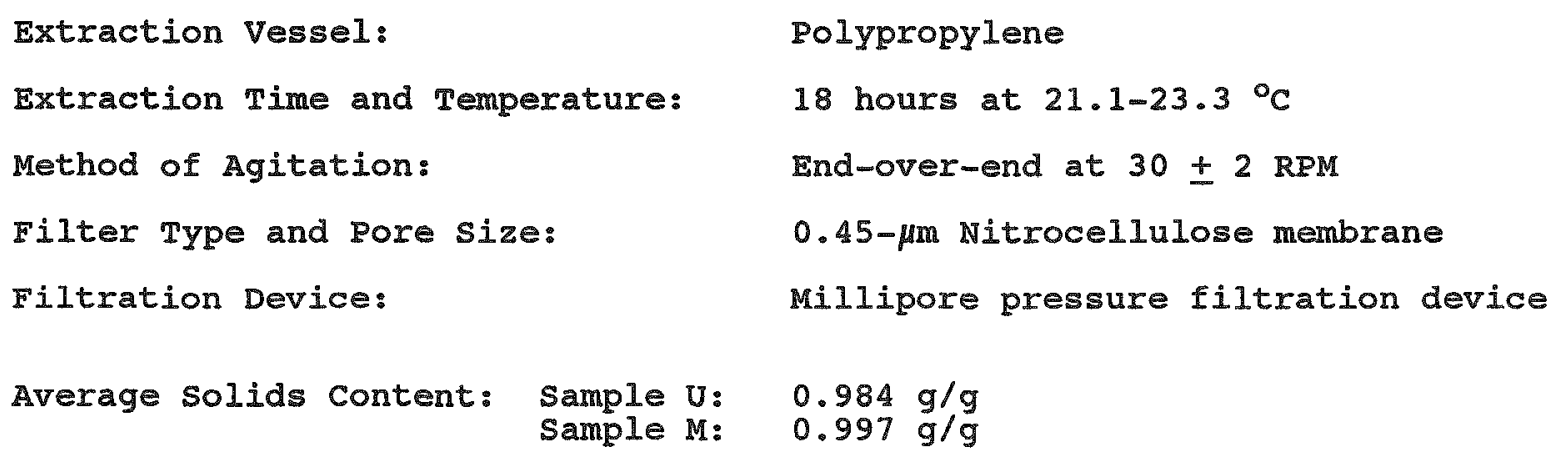

EXTRACTION DATA

WASTE U

\begin{tabular}{|c|c|c|c|}
\hline $\begin{array}{l}\text { In Generating } \\
\text { Extract Number }\end{array}$ & $\begin{array}{l}\text { Average TDS } \\
\text { Content }\end{array}$ & $\begin{array}{l}\text { Mass Lost through } \\
\text { Dissolution, } \mathrm{M}^{\prime} \mathrm{g}\end{array}$ & $\begin{array}{l}\text { EFM for Next } \\
\text { Extraction, } 9\end{array}$ \\
\hline $\begin{array}{l}U-A-1 \\
U-B-1\end{array}$ & $\begin{array}{l}0.15 \\
0\end{array}$ & $\begin{array}{l}0.26 \\
0\end{array}$ & $\begin{array}{l}1,610.9 \\
1,741.7\end{array}$ \\
\hline $\begin{array}{l}U-A-2 \\
U-B-2\end{array}$ & $\begin{array}{l}0 \\
0\end{array}$ & $\begin{array}{l}0 \\
0\end{array}$ & $\begin{array}{l}1,767.8 \\
1,741.9\end{array}$ \\
\hline $\begin{array}{l}U-A-3 \\
U-B-3\end{array}$ & $\begin{array}{l}0 \\
0\end{array}$ & $\begin{array}{l}0 \\
0\end{array}$ & $\begin{array}{l}1,786.2 \\
1,775.3\end{array}$ \\
\hline $\begin{array}{l}U-A-4 \\
U-B-4\end{array}$ & $\begin{array}{l}0 \\
0\end{array}$ & $\begin{array}{l}0 \\
0\end{array}$ & $\begin{array}{l}1,747.4 \\
1,806.3\end{array}$ \\
\hline $\begin{array}{l}U-A-5 \\
U-B-5\end{array}$ & $\begin{array}{l}0 \\
0\end{array}$ & $\begin{array}{l}0 \\
0\end{array}$ & $\begin{array}{l}1,798.7 \\
1,750.0\end{array}$ \\
\hline $\begin{array}{l}U-A-6 \\
U-B-6\end{array}$ & $\begin{array}{l}0 \\
0\end{array}$ & $\begin{array}{l}0 \\
0\end{array}$ & $\begin{array}{l}1,791.6 \\
1,694.7\end{array}$ \\
\hline $\begin{array}{l}U-A-7 \\
U-B-7\end{array}$ & $\begin{array}{l}0 \\
0\end{array}$ & $\begin{array}{l}0 \\
0\end{array}$ & $\begin{array}{l}1,778.8 \\
1,728.6\end{array}$ \\
\hline $\begin{array}{l}U-A-8 \\
U-B-8\end{array}$ & $\begin{array}{l}0 \\
0\end{array}$ & $\begin{array}{l}0 \\
0\end{array}$ & $\begin{array}{l}1,763.7 \\
1,794.1\end{array}$ \\
\hline $\begin{array}{l}U-A-9 \\
U-B-9\end{array}$ & $\begin{array}{l}0 \\
0\end{array}$ & $\begin{array}{l}0 \\
0\end{array}$ & $\begin{array}{l}1,780.7 \\
1.780 .1\end{array}$ \\
\hline $\begin{array}{l}U-A-10 \\
U-B-10\end{array}$ & $\begin{array}{l}0 \\
0\end{array}$ & $\begin{array}{l}0 \\
0\end{array}$ & --- \\
\hline
\end{tabular}




\section{SUMMARY SHEEP -- IABORATORY \#6}

EXTRACTION DATA

WASTE M

\begin{tabular}{|c|c|c|c|}
\hline $\begin{array}{l}\text { In Generating } \\
\text { Extract Number }\end{array}$ & $\begin{array}{l}\text { Average TDS } \\
\text { Content }\end{array}$ & $\begin{array}{l}\text { Mass Lost through } \\
\text { Dissolution, } M_{d} g\end{array}$ & $\begin{array}{l}\text { EFM for Next } \\
\text { Extraction, } 9\end{array}$ \\
\hline $\begin{array}{l}M-A-1 \\
M-B-1\end{array}$ & $\begin{array}{l}0 \\
0\end{array}$ & $\begin{array}{l}0 \\
0\end{array}$ & $\begin{array}{l}1,877.5 \\
1,885.1\end{array}$ \\
\hline $\begin{array}{l}M-A-2 \\
M-B-2\end{array}$ & $\begin{array}{l}0 \\
0\end{array}$ & $\begin{array}{l}0 \\
0\end{array}$ & $\begin{array}{l}1,855.6 \\
1,817.2\end{array}$ \\
\hline $\begin{array}{l}M-A-3 \\
M-B-3\end{array}$ & $\begin{array}{l}0 \\
0\end{array}$ & $\begin{array}{l}0 \\
0\end{array}$ & $\begin{array}{l}1.824 .0 \\
1.828 .3\end{array}$ \\
\hline $\begin{array}{l}M-A-4 \\
M-B-4\end{array}$ & $\begin{array}{l}0 \\
0\end{array}$ & $\begin{array}{l}0 \\
0\end{array}$ & $\begin{array}{l}1,830.7 \\
1,903.8\end{array}$ \\
\hline $\begin{array}{l}M-A-5 \\
M-B-5\end{array}$ & $\begin{array}{l}0 \\
0\end{array}$ & $\begin{array}{l}0 \\
0\end{array}$ & $\begin{array}{l}1,804.8 \\
1,831.2\end{array}$ \\
\hline $\begin{array}{l}M-A-6 \\
M-B-6\end{array}$ & $\begin{array}{l}0 \\
0\end{array}$ & $\begin{array}{l}0 \\
0\end{array}$ & $\begin{array}{l}1,710.2 \\
1,729.9\end{array}$ \\
\hline $\begin{array}{l}M-A-7 \\
M-B-7\end{array}$ & $\begin{array}{l}0 \\
0\end{array}$ & $\begin{array}{l}0 \\
0\end{array}$ & $\begin{array}{l}1,740.7 \\
1.872 .8\end{array}$ \\
\hline $\begin{array}{l}M-A-8 \\
M-B-8\end{array}$ & $\begin{array}{l}0 \\
0\end{array}$ & $\begin{array}{l}0 \\
0\end{array}$ & $\begin{array}{l}1,656.0 \\
1,833.1\end{array}$ \\
\hline $\begin{array}{l}M-A-9 \\
M-B-9\end{array}$ & $\begin{array}{l}0 \\
0\end{array}$ & $\begin{array}{l}0 \\
0\end{array}$ & $\begin{array}{l}1.831 .9 \\
1.745 .1\end{array}$ \\
\hline $\begin{array}{l}M-A-10 \\
M-B-10\end{array}$ & $\begin{array}{l}0 \\
0\end{array}$ & $\begin{array}{l}0 \\
0\end{array}$ & $\begin{array}{l}--- \\
--\infty\end{array}$ \\
\hline
\end{tabular}


SUMMARY SHEET -- LABORATORY \#6

\section{pH VALUES}

Waste U: $p H$ Values of Extraction Fluids and Extracts

\begin{tabular}{lccc} 
Extract & $\begin{array}{c}\text { pH of Extraction } \\
\text { Fluid Used }\end{array}$ & $\begin{array}{c}\text { pH of Extraction slurry } \\
\text { Before Filtration }\end{array}$ & $\begin{array}{c}\text { pH of Extract } \\
\text { After Filtration }\end{array}$ \\
\hline U-A-1 & 4.3 & 11.5 & 11.9 \\
U-B-1 & 4.3 & 11.5 & 11.7 \\
U-A-2 & 4.3 & 11.1 & 11.0 \\
U-B-2 & 4.3 & 11.0 & 11.2 \\
U-A-3 & 4.3 & 10.9 & 11.1 \\
U-B-3 & 4.3 & 11.0 & 11.4 \\
U-A-4 & 4.3 & 10.9 & 11.1 \\
U-B-4 & 4.3 & 10.9 & 11.2 \\
U-A-5 & 4.3 & 10.8 & 11.0 \\
U-B-5 & 4.3 & 10.9 & 11.1 \\
U-A-6 & 4.3 & 10.8 & 10.9 \\
U-B-6 & 4.3 & 10.8 & 11.0 \\
U-A-7 & 4.3 & 10.7 & 10.8 \\
U-B-7 & 4.3 & 10.8 & 10.9 \\
U-A-8 & 4.3 & 10.8 & 10.9 \\
U-B-8 & 4.3 & 10.8 & 11.0 \\
U-A-9 & 4.3 & 10.6 & 10.8 \\
U-B-9 & 4.3 & 10.6 & 10.8 \\
U-A-10 & 4.3 & 10.7 & 10.8 \\
U-B-10 & 4.3 & 10.7 & 10.7 \\
\hline
\end{tabular}




\section{SUMMARY SHEET -- LABORATORY \#6}

\section{PI VALUES}

Waste M: pH Values of Extraction Fluids and Extracts

\begin{tabular}{|c|c|c|c|}
\hline Extract & $\begin{array}{l}\text { pH of Extraction } \\
\text { Fluid Used }\end{array}$ & $\begin{array}{l}\text { pH of Extraction } \\
\text { Slurry Before Filtration }\end{array}$ & $\begin{array}{l}\text { pH of Extract } \\
\text { After Filtration }\end{array}$ \\
\hline $\begin{array}{l}M-A-1 \\
M-B-1\end{array}$ & $\begin{array}{l}5.0 \\
5.0\end{array}$ & $\begin{array}{l}6.0 \\
6.0\end{array}$ & $\begin{array}{l}6.2 \\
6.2\end{array}$ \\
\hline $\begin{array}{l}M-A-2 \\
M-B-2\end{array}$ & $\begin{array}{l}5.0 \\
5.0\end{array}$ & $\begin{array}{l}6.3 \\
6.3\end{array}$ & $\begin{array}{l}6.5 \\
6.4\end{array}$ \\
\hline $\begin{array}{l}M-A-3 \\
M-B-3\end{array}$ & $\begin{array}{l}5.0 \\
5.0\end{array}$ & $\begin{array}{l}6.9 \\
6.7\end{array}$ & $\begin{array}{l}7.2 \\
6.9\end{array}$ \\
\hline $\begin{array}{l}M-A-4 \\
M-B-4\end{array}$ & $\begin{array}{l}5.0 \\
5.0\end{array}$ & $\begin{array}{l}7.7 \\
7.7\end{array}$ & $\begin{array}{l}7.9 \\
7.7\end{array}$ \\
\hline $\begin{array}{l}M-A-5 \\
M-B-5\end{array}$ & $\begin{array}{l}5.0 \\
5.0\end{array}$ & $\begin{array}{l}8.0 \\
8.0\end{array}$ & $\begin{array}{l}7.3 \\
7.8\end{array}$ \\
\hline $\begin{array}{l}M-A-6 \\
M-B-6\end{array}$ & $\begin{array}{l}5.0 \\
5.0\end{array}$ & $\begin{array}{l}8.1 \\
8.1\end{array}$ & $\begin{array}{l}8.2 \\
8.1\end{array}$ \\
\hline $\begin{array}{l}\mathrm{M}-\mathrm{A}-7 \\
\mathrm{M}-\mathrm{B}-7\end{array}$ & $\begin{array}{l}5.0 \\
5.0\end{array}$ & $\begin{array}{l}8.3 \\
8.3\end{array}$ & $\begin{array}{l}8.3 \\
8.2\end{array}$ \\
\hline $\begin{array}{l}\mathrm{M}-\mathrm{A}-8 \\
\mathrm{M}-\mathrm{B}-8\end{array}$ & $\begin{array}{l}5.0 \\
5.0\end{array}$ & $\begin{array}{l}8.4 \\
8.3\end{array}$ & $\begin{array}{l}8.5 \\
8.5\end{array}$ \\
\hline $\begin{array}{l}\text { M-A-9 } \\
\text { M-B-9 }\end{array}$ & $\begin{array}{l}5.0 \\
5.0\end{array}$ & $\begin{array}{l}8.4 \\
8.3\end{array}$ & $\begin{array}{l}8.5 \\
8.5\end{array}$ \\
\hline $\begin{array}{l}M-A-10 \\
M-B-10\end{array}$ & $\begin{array}{l}5.0 \\
5.0\end{array}$ & $\begin{array}{l}8.3 \\
8.3\end{array}$ & $\begin{array}{l}8.2 \\
8.2\end{array}$ \\
\hline
\end{tabular}


None of the samples were digested prior to analysis. All samples were analyzed by ICP using SW846 Method 6010 .

\section{DEVIATIONS FROM THE SPECIFIED PROCEDURES}

1. Despite instructions given to the participants in the interlaboratory study specifications to start the first extraction step on Monday and to stop the extraction sequence over the weekend, laboratory \#6 started the extractions on Tuesday and continued them through the weekend.

2. Section 9.6 of the method specifies that the weighings for TDS determination are to be made to $+0.1 \mathrm{mg}$ : however, laboratory \#6 performed their weighings for TDS determination using a balance weighing to only a tenth of a gram, which reduced the sensitivity of their determinations.

3. For extraction step $U-\mathrm{A}-1$, the TDS calculation is incorrect. The person performing the calculations failed to divide by 10 in calculating TDS. As a result, the $\mathrm{M}_{\mathrm{g}} \mathrm{M}_{\mathrm{S}}$, and EFM values calculated for that step are incorrect. The calculated TDs value for the rest of the U-A extraction steps was 0 . As a result, the incorrect $M_{s}$ value from U-A-1, $99.7 \mathrm{~g}$, was used in the rest of the U-A calculations for EFM. The correct value for $\mathrm{M}_{\mathrm{s}}$, based on this laboratory's data in step U-A-1, is $100.0 \mathrm{~g}$. The correct EFM value and the EFM that was added for each step are listed below.

Amount Added

$U-A-1$
$U-A-2$
$U-A-3$
$U-A-4$
$U-A-5$
$U-A-6$
$U-A-7$
$U-A-8$
$U-A-9$

$$
\begin{aligned}
& 1,610.9 \mathrm{~g} \\
& 1,767.8 \mathrm{~g} \\
& 1,786.29 \\
& 1,747.49 \\
& 1,798.7 \mathrm{~g} \\
& 1,791.69^{\mathrm{b}} \\
& 1,778.89 \\
& 1,763.7 \mathrm{~g} \\
& 1,780.7 \mathrm{~g}^{\mathrm{c}}
\end{aligned}
$$

a This is based on the data generated in extraction step U-A-1 by laboratory \#6.

b Additional error in step $U-A-6$ in calculating this value

c Additional error in step U-A-9 in calculating this value 
Extraction Vessel:

Extraction Time and Temperature:

Method of Agitation:

Filter Type and Pore size:

Filtration Device:

Average Solids Content:
Borosilicate glass with teflon lined lids/high density polyethylene

Waste U: 18 hours; extraction temperature not recorded

Waste M: 18 hours; extraction temperature range $18-21^{\circ} \mathrm{C}$

$30 \mathrm{rpm}$ rotary extractor

$0.7-\mu \mathrm{m}$ borosilicate filter

1.5-1iter stainless steel filtration system
Sample U:
$\begin{array}{ll}0.982 & \mathrm{~g} / \mathrm{g} \\ 0.9982 \mathrm{~g} / \mathrm{g}\end{array}$
Sample M:

EXIRACPION DATA

WASTE U

\begin{tabular}{|c|c|c|c|}
\hline $\begin{array}{l}\text { In Generating } \\
\text { Extract Number }\end{array}$ & $\begin{array}{l}\text { Average TDS } \\
\text { Content }\end{array}$ & $\begin{array}{l}\text { Mass Lost through } \\
\text { Dissolution, } \mathrm{M}_{\mathrm{d}} \mathrm{g}\end{array}$ & $\begin{array}{l}\text { EFM for Next } \\
\text { Extraction, g }\end{array}$ \\
\hline $\begin{array}{l}\mathrm{U}-\mathrm{A}-1 \\
\mathrm{U}-\mathrm{B}-1\end{array}$ & $\begin{array}{l}0.001 \\
0.001\end{array}$ & $\begin{array}{l}0.002 \\
0.002\end{array}$ & $\begin{array}{l}1,831 \\
1,774.93\end{array}$ \\
\hline $\begin{array}{l}U-A-2 \\
U-B-2\end{array}$ & $\begin{array}{l}0.001 \\
0.001\end{array}$ & $\begin{array}{l}0.001 \\
0.001\end{array}$ & $\begin{array}{l}1,733.82 \\
1,726.78\end{array}$ \\
\hline $\begin{array}{l}U-A-3 \\
U-B-3\end{array}$ & $\begin{array}{l}0 \\
0\end{array}$ & $\stackrel{0}{0}$ & $1,697 \cdot 34$ \\
\hline $\begin{array}{l}U-A-4 \\
U-B-4\end{array}$ & $\begin{array}{l}0 \\
0\end{array}$ & $\begin{array}{l}0 \\
0\end{array}$ & $\begin{array}{l}1,738.96 \\
1,765.96\end{array}$ \\
\hline $\begin{array}{l}\mathrm{U}-\mathrm{A}-5 \\
\mathrm{U}-\mathrm{B}-5\end{array}$ & $\begin{array}{l}0 \\
0\end{array}$ & $\begin{array}{l}0 \\
0\end{array}$ & $\begin{array}{l}1,694.98 \\
1,767.06\end{array}$ \\
\hline $\begin{array}{l}U-A-6 \\
U-B-6\end{array}$ & $\begin{array}{l}0 \\
0\end{array}$ & $\begin{array}{l}0 \\
0\end{array}$ & $\begin{array}{l}1,745.44 \\
1,630.53\end{array}$ \\
\hline $\begin{array}{l}\mathrm{U}-\mathrm{A}-7 \\
\mathrm{U}-\mathrm{B}-7\end{array}$ & $\begin{array}{l}0 \\
0\end{array}$ & $\begin{array}{l}0 \\
0\end{array}$ & $\begin{array}{l}1,755 \cdot 15 \\
1,784.60\end{array}$ \\
\hline $\begin{array}{l}U-A-8 \\
U-B-8\end{array}$ & $\begin{array}{l}0 \\
0\end{array}$ & $\begin{array}{l}0 \\
0\end{array}$ & $\begin{array}{l}1,733.63 \\
1,759.48\end{array}$ \\
\hline $\begin{array}{l}U-A-9 \\
U-B-9\end{array}$ & $\begin{array}{l}0 \\
0\end{array}$ & $\stackrel{0}{0}$ & $\begin{array}{l}1,770 \cdot 54 \\
1,764.64\end{array}$ \\
\hline $\begin{array}{l}U-A-10 \\
U-B-10\end{array}$ & $\begin{array}{l}0 \\
0\end{array}$ & $\begin{array}{l}0 \\
0\end{array}$ & $=-$ \\
\hline
\end{tabular}


SUMMARY SHEET - LABORATORY 唶7

EXTRACTION DATA

WASTE M

\begin{tabular}{|c|c|c|c|}
\hline $\begin{array}{l}\text { In Generating } \\
\text { Extract Number }\end{array}$ & $\begin{array}{l}\text { Average TDS } \\
\text { Content }\end{array}$ & $\begin{array}{l}\text { Mass Lost through } \\
\text { Dissolution, } \mathrm{M}^{\prime} \mathrm{g}\end{array}$ & $\begin{array}{l}\text { EFM for Next } \\
\text { Extraction, } g\end{array}$ \\
\hline $\begin{array}{l}M-A-1 \\
M-B-1\end{array}$ & $\begin{array}{l}0.0005 \\
0.0005\end{array}$ & $\begin{array}{l}0.0009 \\
0.0009\end{array}$ & $\begin{array}{l}1,872.6 \\
1,855.5\end{array}$ \\
\hline $\begin{array}{l}M-A-2 \\
M-B-2\end{array}$ & $\begin{array}{l}0.0002 \\
0.0002\end{array}$ & $\begin{array}{l}0.0003 \\
0.0004\end{array}$ & $\begin{array}{l}1,832.3 \\
1,889.7\end{array}$ \\
\hline $\begin{array}{l}M-A-3 \\
M-B-3\end{array}$ & $\begin{array}{l}0 \\
0\end{array}$ & $\begin{array}{l}0 \\
0\end{array}$ & $\begin{array}{l}1,855.5 \\
1,877.1\end{array}$ \\
\hline $\begin{array}{l}M-A-4 \\
M-B-4\end{array}$ & $\begin{array}{l}0 \\
0\end{array}$ & $\begin{array}{l}0 \\
0\end{array}$ & $\begin{array}{l}1,868.3 \\
1,865.3\end{array}$ \\
\hline $\begin{array}{l}M-A-5 \\
M-B-5\end{array}$ & $\begin{array}{l}0 \\
0\end{array}$ & $\begin{array}{l}0 \\
0\end{array}$ & $\begin{array}{l}1,859.1 \\
1,882.3\end{array}$ \\
\hline $\begin{array}{l}M-A-6 \\
M-B-6\end{array}$ & $\begin{array}{l}0 \\
0\end{array}$ & $\begin{array}{l}0 \\
0\end{array}$ & $\begin{array}{l}1,830.4 \\
1,832.0\end{array}$ \\
\hline $\begin{array}{l}M-A-7 \\
M-B-7\end{array}$ & $\begin{array}{l}0 \\
0\end{array}$ & $\begin{array}{l}0 \\
0\end{array}$ & $\begin{array}{l}1,852.6 \\
1,852.5\end{array}$ \\
\hline $\begin{array}{l}M-A-8 \\
M-B-8\end{array}$ & $\begin{array}{l}0 \\
0\end{array}$ & $\begin{array}{l}0 \\
0\end{array}$ & $\begin{array}{l}1,883.2 \\
1,861.7\end{array}$ \\
\hline $\begin{array}{l}M-A-9 \\
M-B-9\end{array}$ & $\begin{array}{l}0 \\
0\end{array}$ & $\begin{array}{l}0 \\
0\end{array}$ & $\begin{array}{l}1,891.2 \\
1,873,2\end{array}$ \\
\hline $\begin{array}{l}M-A-10 \\
M-B-10\end{array}$ & $\begin{array}{l}0 \\
0\end{array}$ & $\begin{array}{l}0 \\
0\end{array}$ & --- \\
\hline
\end{tabular}


SUMMARY SHEET -- LABORATORY \#7

\section{PH VALUES}

Waste U: $\mathrm{pH}$ Values of Extraction Fluids and Extracts

\begin{tabular}{lccc} 
Extract & $\begin{array}{c}\text { pH of Extraction } \\
\text { Fluid Used }\end{array}$ & $\begin{array}{c}\text { pH of Extraction slurry } \\
\text { Before Filtration }\end{array}$ & $\begin{array}{c}\text { pH of Extract } \\
\text { After Filtration }\end{array}$ \\
\hline U-A-1 & 4.3 & 11.3 & 11.3 \\
U-B-1 & 4.3 & 11.4 & 11.4 \\
U-A-2 & 4.3 & 10.8 & 10.9 \\
U-B-2 & 4.3 & 10.8 & 10.9 \\
U-A-3 & 4.3 & 10.6 & 10.6 \\
U-B-3 & 4.3 & 10.7 & 10.7 \\
U-A-4 & 4.3 & 10.5 & 10.5 \\
U-B-4 & 4.3 & 10.5 & 10.5 \\
U-A-5 & 4.3 & 10.3 & 10.3 \\
U-B-5 & 4.3 & 10.3 & 10.3 \\
U-A-6 & 4.3 & 10.4 & 10.6 \\
U-B-6 & 4.3 & 10.6 & 10.7 \\
U-A-7 & 4.3 & 10.2 & 10.4 \\
U-B-7 & 4.3 & 10.2 & 10.2 \\
U-A-8 & 4.3 & 10.1 & 10.2 \\
U-B-8 & 4.3 & 10.1 & 10.2 \\
U-A-9 & 4.3 & 9.9 & 10.0 \\
U-B-9 & 4.3 & 10.1 & 10.2 \\
U-A-10 & 4.3 & 10.5 & 10.3 \\
U-B-10 & 4.3 & 10.5 & 10.2 \\
\hline & & & \\
\hline
\end{tabular}


SUMMARY SHEET -- LABORATORY H7

\section{pH VALUES}

Waste M: $\mathrm{pH}$ Values of Extraction Fluids and Extracts

\begin{tabular}{lccc} 
Extract & $\begin{array}{c}\text { pH of Extraction } \\
\text { Fluid Used }\end{array}$ & $\begin{array}{c}\text { pH of Extraction } \\
\text { Slurry Before Filtration }\end{array}$ & $\begin{array}{c}\text { pH of Extract } \\
\text { After Filtration }\end{array}$ \\
\hline M-A-1 & 5.0 & 6.0 & 6.0 \\
M-B-1 & 5.0 & 6.1 & 6.2 \\
M-A-2 & 5.0 & 6.2 & 6.3 \\
M-B-2 & 5.0 & 6.3 & 6.3 \\
M-A-3 & 5.0 & 6.4 & 6.4 \\
M-B-3 & 5.0 & 6.5 & 6.3 \\
M-A-4 & 5.0 & 7.0 & 7.1 \\
M-B-4 & 5.0 & 7.1 & 7.1 \\
M-A-5 & 5.0 & 8.1 & 7.6 \\
M-B-5 & 5.0 & 7.8 & 7.3 \\
M-A-6 & 5.0 & 8.3 & 7.9 \\
M-B-6 & 5.0 & 8.3 & 7.7 \\
M-A-7 & 5.0 & 8.4 & 7.9 \\
M-B-7 & 5.0 & 8.4 & 7.8 \\
M-A-8 & 5.0 & 8.5 & 7.8 \\
M-B-8 & 5.0 & 8.5 & 7.7 \\
M-A-9 & 5.0 & 8.5 & 8.0 \\
M-B-9 & 5.0 & 8.4 & 7.9 \\
M-A-10 & 5.0 & 8.7 & 8.1 \\
M-B-10 & 5.0 & 8.7 & 8.1 \\
\hline & & & \\
\hline
\end{tabular}


SUMMARY SHEET $=-$ IABORATORY \#7

ANATYTICAL INFORMATION

The samples were digested prior to analysis using EPA Method 3010 . All samples were analyzed by ICP using sw846 Method 6010.

\section{DEVIATIONS FROM THE SPECIFIED PROCEDURES}

1. $0.7-\mu \mathrm{m}$ borosilicate filters were used for filtering instead of the

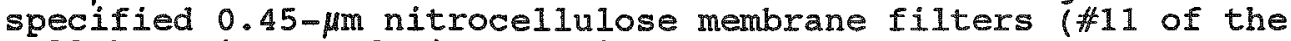
collaborative study instructions).

2. Some mass measurements for determining average solids content were made to only tenths and hundredths of a gram.

3. TDS determinations were not performed in duplicate.

4. For extraction U-A-1, EFM was calculated incorrectly. The extraction fluid mass that was calculated was $1.988 .06 \mathrm{~g}$, and the correct amount is $1,998.06 \mathrm{~g}$.

5. For extraction U-B-3, EFM was calculated incorrectly. The extraction fluid mass that was calculated was $1,697.3 \mathrm{~g}$, and the correct amount is $1,663.6 \mathrm{~g}$. 
Extraction Vessel:

Extraction Iime and Temperature:

Method of Agitation:

Filter Type and Pore size:

Filtration Device:

Average Solids Content: Sample U Sample M:
Acid-washed plastic bottles (1-gallon capacity) with teflon-lined plastic caps

$17.75-18.25$ hours at $21-28^{\circ} \mathrm{C}$

End-over-end rotation using rotary extractor at -30 RPM

$0.45-\mu \mathrm{m}$ Nitrocellulose filter

1.5-Liter pressure filtration device with pre-purified nitrogen gas

EXTRACTION DATA

WASTE U

\begin{tabular}{|c|c|c|c|}
\hline $\begin{array}{l}\text { In cenerating } \\
\text { Extract Number }\end{array}$ & $\begin{array}{l}\text { Average TDS } \\
\text { Content }\end{array}$ & $\begin{array}{l}\text { Mass Lost through } \\
\text { Dissolution, } \mathrm{M}^{\prime} \mathrm{g}\end{array}$ & $\begin{array}{l}\text { EFM for Next } \\
\text { Extraction, } 9\end{array}$ \\
\hline $\begin{array}{l}U-A-1 \\
U-B-1\end{array}$ & $\begin{array}{l}0 \\
0\end{array}$ & $\begin{array}{l}0 \\
0\end{array}$ & $\begin{array}{l}1,722 \cdot 9 \\
1,727.1\end{array}$ \\
\hline $\begin{array}{l}U-A-2 \\
U-B-2\end{array}$ & $\begin{array}{l}5 \cdot 6 \\
4 \cdot 8\end{array}$ & $\begin{array}{r}10.7 \\
9.1\end{array}$ & $\begin{array}{l}1,538.8 \\
1,495.0\end{array}$ \\
\hline $\begin{array}{l}U-A-3 \\
U-B-3\end{array}$ & $\begin{array}{l}4 \cdot 2 \\
4 \cdot 2\end{array}$ & $\begin{array}{l}8.0 \\
7.9\end{array}$ & $\begin{array}{l}1,317.8 \\
1,369.8\end{array}$ \\
\hline $\begin{array}{l}U-A-4 \\
U-B-4\end{array}$ & $\begin{array}{l}0 \\
0\end{array}$ & $\begin{array}{l}0 \\
0\end{array}$ & $\begin{array}{l}1,300.4 \\
1,360.3\end{array}$ \\
\hline $\begin{array}{l}U-A-5 \\
U-B-5\end{array}$ & $\begin{array}{l}0 \\
0\end{array}$ & $\begin{array}{l}0 \\
0\end{array}$ & $\begin{array}{l}1,199 \cdot 5 \\
1,244.2\end{array}$ \\
\hline $\begin{array}{l}U-A-6 \\
U-B-6\end{array}$ & $\begin{array}{l}0 \\
0\end{array}$ & $\begin{array}{l}0 \\
0\end{array}$ & $\begin{array}{l}1,323.9 \\
1,330.6\end{array}$ \\
\hline $\begin{array}{l}U-A-7 \\
U-B-7\end{array}$ & $\begin{array}{l}0 \\
0\end{array}$ & $\begin{array}{l}0 \\
0\end{array}$ & $\begin{array}{l}1,300.0 \\
1,333.4\end{array}$ \\
\hline $\begin{array}{l}U-A-8 \\
U-B-8\end{array}$ & $\begin{array}{l}0 \\
0\end{array}$ & $\begin{array}{l}0 \\
0\end{array}$ & $\begin{array}{l}1,273.8 \\
1,319.7\end{array}$ \\
\hline $\begin{array}{l}U-A-9 \\
U-B-9\end{array}$ & $\begin{array}{l}0 \\
0\end{array}$ & $\begin{array}{l}0 \\
0\end{array}$ & $\begin{array}{l}1,244.8 \\
1,342.7\end{array}$ \\
\hline $\begin{array}{l}U-A-10 \\
U-B-10\end{array}$ & $\begin{array}{l}0 \\
0\end{array}$ & $\begin{array}{l}0 \\
0\end{array}$ & $\begin{array}{l}--- \\
---\end{array}$ \\
\hline
\end{tabular}


EXIRACTION DAPA

WASTE M

\begin{tabular}{|c|c|c|c|}
\hline $\begin{array}{l}\text { In Generating } \\
\text { Extract Number }\end{array}$ & $\begin{array}{l}\text { Average TDS } \\
\text { Content }\end{array}$ & $\begin{array}{l}\text { Mass Lost through } \\
\text { Dissolution, } M_{d} g\end{array}$ & $\begin{array}{l}\text { EFM for Next } \\
\text { Extraction, } g\end{array}$ \\
\hline $\begin{array}{l}\mathrm{M}-\mathrm{A}-1 \\
\mathrm{M}-\mathrm{B}-1\end{array}$ & $\begin{array}{l}0 \\
0\end{array}$ & $\begin{array}{l}0 \\
0\end{array}$ & $\begin{array}{l}1,486.8 \\
1,797.4\end{array}$ \\
\hline $\begin{array}{l}M-A-2 \\
M-B-2\end{array}$ & $\begin{array}{l}1.1 \\
1.5\end{array}$ & $\begin{array}{l}2.2 \\
2.9\end{array}$ & $\begin{array}{l}1,653.6 \\
1,634.9\end{array}$ \\
\hline $\begin{array}{l}M-A-3 \\
M-B-3\end{array}$ & $\begin{array}{l}0.7 \\
0.6\end{array}$ & $\begin{array}{l}1.4 \\
1.2\end{array}$ & $\begin{array}{l}1,607.0 \\
1,404.3\end{array}$ \\
\hline $\begin{array}{l}M-A-4 \\
M-B-4\end{array}$ & $\begin{array}{l}0 \\
0\end{array}$ & $\begin{array}{l}0 \\
0\end{array}$ & $\begin{array}{l}1,547.5 \\
1,505.2\end{array}$ \\
\hline $\begin{array}{l}M-A-5 \\
M-B-5\end{array}$ & $\begin{array}{l}0 \\
0\end{array}$ & $\begin{array}{l}0 \\
0\end{array}$ & $\begin{array}{l}1,613.8 \\
1,494.6\end{array}$ \\
\hline $\begin{array}{l}M-A-6 \\
M-B-6\end{array}$ & $\begin{array}{l}0 \\
0\end{array}$ & $\begin{array}{l}0 \\
0\end{array}$ & $\begin{array}{l}1,535 \cdot 1 \\
1,555.4\end{array}$ \\
\hline $\begin{array}{l}M-A-7 \\
M-B-7\end{array}$ & $\begin{array}{l}0 \\
0\end{array}$ & $\begin{array}{l}0 \\
0\end{array}$ & $\begin{array}{l}1,550.6 \\
1,490.1\end{array}$ \\
\hline $\begin{array}{l}M-A-8 \\
M-B-8\end{array}$ & $\begin{array}{l}0 \\
0\end{array}$ & $\begin{array}{l}0 \\
0\end{array}$ & $\begin{array}{l}1,533 \cdot 5 \\
1,584.3\end{array}$ \\
\hline $\begin{array}{l}\text { M-A-9 } \\
\text { M-B-9 }\end{array}$ & $\begin{array}{l}0 \\
0\end{array}$ & $\begin{array}{l}0 \\
0\end{array}$ & $\begin{array}{l}1,568.9 \\
1,581.8\end{array}$ \\
\hline $\begin{array}{l}M-A-10 \\
M-B-10\end{array}$ & $\begin{array}{l}0 \\
0\end{array}$ & $\begin{array}{l}0 \\
0\end{array}$ & $\overline{--}$ \\
\hline
\end{tabular}


SUMMARY SHEET - - LABORATORY \#8

\section{PH VALUES}

Waste U: pH Values of Extraction Fluids and Extracts

\begin{tabular}{|c|c|c|c|}
\hline Extract & $\begin{array}{l}\text { pH of Extraction } \\
\text { Fluid Used }\end{array}$ & $\begin{array}{l}\text { pH of Extraction slurry } \\
\text { Before Filtration }\end{array}$ & $\begin{array}{l}\text { pH of Extract } \\
\text { After Filtration }\end{array}$ \\
\hline $\begin{array}{l}U-A-1 \\
U-B-1\end{array}$ & $\begin{array}{l}4 \cdot 3 \\
4.3\end{array}$ & $\begin{array}{l}11.1 \\
11.0\end{array}$ & $\begin{array}{l}11.1 \\
10.9\end{array}$ \\
\hline $\begin{array}{l}U-A-2 \\
U-B-2\end{array}$ & $\begin{array}{l}4 \cdot 3 \\
4.3\end{array}$ & $\begin{array}{l}11.5 \\
11.5\end{array}$ & $\begin{array}{l}11.5 \\
11.5\end{array}$ \\
\hline $\begin{array}{l}U-A-3 \\
U-B-3\end{array}$ & $\begin{array}{l}4 \cdot 3 \\
4 \cdot 3\end{array}$ & $\begin{array}{l}11.4 \\
11.4\end{array}$ & $\begin{array}{l}11 \cdot 4 \\
11.4\end{array}$ \\
\hline $\begin{array}{l}\mathrm{U}-\mathrm{A}-4 \\
\mathrm{U}-\mathrm{B}-4\end{array}$ & $\begin{array}{l}4 \cdot 3 \\
4 \cdot 3\end{array}$ & $\begin{array}{l}11.4 \\
11.4\end{array}$ & $\begin{array}{l}11.4 \\
11.4\end{array}$ \\
\hline $\begin{array}{l}U-A-5 \\
U-B-5\end{array}$ & $\begin{array}{l}4 \cdot 3 \\
4 \cdot 3\end{array}$ & $\begin{array}{l}11.3 \\
11.3\end{array}$ & $\begin{array}{l}11.3 \\
11.3\end{array}$ \\
\hline $\begin{array}{l}U-A-6 \\
U-B-6\end{array}$ & $\begin{array}{l}4 \cdot 3 \\
4.3\end{array}$ & $\begin{array}{l}11.2 \\
11.2\end{array}$ & $\begin{array}{l}11.2 \\
11.2\end{array}$ \\
\hline $\begin{array}{l}U-A-7 \\
U-B-7\end{array}$ & $\begin{array}{l}4 \cdot 3 \\
4 \cdot 3\end{array}$ & $\begin{array}{l}11.2 \\
11.2\end{array}$ & $\begin{array}{l}11.2 \\
11.2\end{array}$ \\
\hline $\begin{array}{l}U-A-8 \\
U-B-8\end{array}$ & $\begin{array}{l}4 \cdot 3 \\
4.3\end{array}$ & $\begin{array}{l}11.2 \\
11.2\end{array}$ & $\begin{array}{l}11.2 \\
11.2\end{array}$ \\
\hline $\begin{array}{l}U-A-9 \\
U-B-9\end{array}$ & $\begin{array}{l}4.3 \\
4.3\end{array}$ & $\begin{array}{l}11.2 \\
11.1\end{array}$ & $\begin{array}{l}11.1 \\
11.1\end{array}$ \\
\hline $\begin{array}{l}U-A-10 \\
U-B-10\end{array}$ & $\begin{array}{l}4.3 \\
4.3\end{array}$ & $\begin{array}{l}11.1 \\
11.1\end{array}$ & $\begin{array}{l}11.1 \\
11.1\end{array}$ \\
\hline
\end{tabular}


SUMMARY SHEET -- LABORATORY \#8

\section{PH VAIUES}

Waste M: pH Values of Extraction Fluids and Extracts

\begin{tabular}{lccc} 
Extract & $\begin{array}{c}\text { pH of Extraction } \\
\text { Fluid Used }\end{array}$ & $\begin{array}{c}\text { pH of Extraction } \\
\text { Slurry }\end{array}$ & $\begin{array}{c}\text { pH of Extract } \\
\text { After Filtration }\end{array}$ \\
\hline M-A-1 & 5.0 & 6.4 & 6.6 \\
M-B-1 & 5.0 & 6.3 & 7.0 \\
M-A-2 & 5.0 & 5.9 & 6.1 \\
M-B-2 & 5.0 & 6.0 & 6.3 \\
M-A-3 & 5.0 & 7.5 & 7.0 \\
M-B-3 & 5.0 & 7.6 & 7.9 \\
M-A-4 & 5.0 & 8.0 & 7.0 \\
M-B-4 & 5.0 & 8.0 & 7.5 \\
M-A-5 & 5.0 & 8.3 & 7.5 \\
M-B-5 & 5.0 & 8.3 & 7.6 \\
M-A-6 & 5.0 & 8.2 & 8.6 \\
M-B-6 & 5.0 & 8.3 & 8.9 \\
M-A-7 & 5.0 & 8.6 & 8.0 \\
M-B-7 & 5.0 & 8.6 & 8.0 \\
M-A-8 & 5.0 & 8.9 & 8.4 \\
M-B-8 & 5.0 & 8.9 & 8.1 \\
M-A-9 & 5.0 & 8.4 & 8.2 \\
M-B-9 & 5.0 & 8.3 & 8.1 \\
M-A-10 & 5.0 & 8.3 & 7.9 \\
M-B-10 & 5.0 & 8.5 & 8.1 \\
\hline
\end{tabular}




\section{MNALYTICAL INFORMATION}

The extracts were digested using sw846 Method 3020 prior to analysis. All of the samples were analyzed by ICP using SW846 Method 6010 .

\section{DEVIATIONS FROM THE SPECIFIED PROCEDURES}

1. This laboratory started the extractions on Monday; however, the extraction sequence was stopped on Tuesday and then started again on Wednesday. Laboratory \#8 also stopped the extraction sequence between extractions 7 and 8 for three days, instead of two days, over a threeday weekend.

2. An error was made by the laboratory in calculating the average solids content for both waste $U$ and waste $M$. In both cases, the numbers were inverted.

Solids content

Calculated Amount Correct Amount

$\begin{array}{lll}\text { Waste U } & 1.019 \mathrm{~g} / \mathrm{g} & .9810 \mathrm{~g} / \mathrm{g} \\ \text { Waste M } & 1.003 \mathrm{~g} / \mathrm{g} & .9966 \mathrm{~g} / \mathrm{g}\end{array}$

3. For both waste $U$ and waste $M$, errors were made in calculating the total dissolved solids content (TDS) of the extracts. These resulted in the addition of incorrect amounts of extraction fluid. The correct values and the values calculated by the laboratory (amount added) are listed below.

Amount Added

$\mathrm{U}-\mathrm{A}-2$

$\mathrm{U}-\mathrm{B}-2$

$\mathrm{U}-\mathrm{A}-3$

$\mathrm{U}-\mathrm{B}-3$

$U-A-4$

$\mathrm{U}-\mathrm{B}-4$

$U-\mathbb{A}-5$

$\mathrm{U}-\mathrm{B}-5$

U-A -6

$\mathrm{U}-\mathrm{B}-6$

$U-A-7$

$\mathrm{U}-\mathrm{B}-7$

$U-A-8$

U-B-8

$\mathbf{U}-\mathbf{A}-9$

$U-B-9$

$$
\begin{aligned}
& 1,538.89 \\
& 1,495.09 \\
& 1,317.89 \\
& 1,369.89 \\
& 1,300.49 \\
& 1,360.39 \\
& 1,199.59 \\
& 1,244.29 \\
& 1,323.99 \\
& 1,330.69 \\
& 1,300.09 \\
& 1,333.49 \\
& 1,273.89 \\
& 1,319.79 \\
& 1,244.89 \\
& 1,342.79
\end{aligned}
$$

$1,764.5 \mathrm{~g}$

$1,686.1 \mathrm{~g}$

$1,711.8 \mathrm{~g}$

$1,726.8 \mathrm{~g}$

$1,694.4 \mathrm{~g}$

$1,717.3 \mathrm{~g}$

$1,593.5 \mathrm{~g}$

$1,601.2 \mathrm{~g}$

$1,717.9 \mathrm{~g}$

$1,687.6 \mathrm{~g}$

$1,694.89$

$1,690.4 \mathrm{~g}$

$1,667.8 \mathrm{~g}$

$1,676.7 \mathrm{~g}$

$1,639.69$

$1,699.7 \mathrm{~g}$ 
(3. cont'd.)

Amount Added

$$
\begin{aligned}
& M-A-2 \\
& M-B-2 \\
& M-A-3 \\
& M-B-3 \\
& M-A-4 \\
& M-B-4 \\
& M-A-5 \\
& M-B-5 \\
& M-A-6 \\
& M-B-6 \\
& M-A-7 \\
& M-B-7 \\
& M-A-8 \\
& M-B-8 \\
& M-A-9 \\
& M-B-9
\end{aligned}
$$

$$
\begin{aligned}
& 1,653.6 \mathrm{~g} \\
& 1,634.9 \mathrm{~g} \\
& 1,607.09 \\
& 1,404.3 \mathrm{~g} \\
& 1,547.5 \mathrm{~g} \\
& 1,505.2 \mathrm{~g} \\
& 1,613.8 \mathrm{~g} \\
& 1,494.6 \mathrm{~g} \\
& 1,535.1 \mathrm{~g} \\
& 1,555.4 \mathrm{~g} \\
& 1,550.6 \mathrm{~g} \\
& 1,490.1 \mathrm{~g} \\
& 1,533.5 \mathrm{~g} \\
& 1,584.3 \mathrm{~g} \\
& 1,568.9 \mathrm{~g} \\
& 1,581.8 \mathrm{~g}
\end{aligned}
$$

Correct Amount

$$
\begin{aligned}
& 1,699.8 \mathrm{~g} \\
& 1,695.8 \mathrm{~g} \\
& 1,697.3 \mathrm{~g} \\
& 1,490.4 \mathrm{~g} \\
& 1,637.8 \mathrm{~g} \\
& 1,591.3 \mathrm{~g} \\
& 1,704.0 \mathrm{~g} \\
& 1,580.7 \mathrm{~g} \\
& 1,525.5 \mathrm{~g} \\
& 1,641.5 \mathrm{~g} \\
& 1,640.8 \mathrm{~g} \\
& 1,576.2 \mathrm{~g} \\
& 1,623.8 \mathrm{~g} \\
& 1,670.4 \mathrm{~g} \\
& 1,659.2 \mathrm{~g} \\
& 1,667.9 \mathrm{~g}
\end{aligned}
$$

4. In determining the TDS content of the first extract for samples U-A, U$B$, M-A, and $M-B$, a balance weighing to only 0.1 grams was used.

5. After extraction \#3 for each of the extraction samples, TDS determination was not continued. Based on the incorrect calculations of the laboratory, TDS determination should have continued. However, based on the correct calculations, this determination should have been stopped. Also, the procedure specifies that TDS determination be stopped after the second extraction, if the requirements stated in the procedure are met. 


\section{APPENDIX F}

ASTM Method D5284-92, Standard Test Method for Sequential Batch Extraction of waste with Mcidic Extraction Fluid 


\title{
Standard Test Method for Sequential Batch Extraction of Waste with Acidic Extraction Fluid"
}

\begin{abstract}
This standard is issued under the fixed desunatuon D 5284: the number immediately following the designation indicates the year of orizgnal adoptson or, in the caste of revision. the sear of last reviston. A number in parentheses nndicates the yeat of lass reapproval. A superscrpt epston lol indicates an edutonal change suce the last revision or reapproval.
\end{abstract}

\section{Scope}

1.1 This test method provides a procedure for the sequen. tial leaching of a waste containing at least $5 \%$ dry solids in order to generate solutions to be used to determine the constituents leached under the specified testing conditions.

1.2 This test method calls for the staking of a known weight of waste with acidic extraction fluid of a specified composition as well as the separation of the liquid phase for analysis. The $\mathrm{pH}$ of the extraction fluid is to reflect the $\mathrm{pH}$ of acidic precipitation in the geographic region in which the waste being tested is to be disposed. The procedure is conducted ten times in sequence on the same sample of waste, and it generates ten solutions.

1.3 This test method is intended to describe the procedure for performing sequential batch extractions only. It does not describe all types of sampling and analytical requirements that may be associated with its application.

1.4 The values stated in SI units are to be regarded as the standard.

1.5 This standard does not purport to address all of the safety problems. if any, associated with its use. It is the responsibility of the user of this standard to establish appropriate safety and heaith practices and determine the applicability of regulatory limitations prior to use.

\section{Referenced Documents}

\subsection{ASTM Siandards:}

D 75 Practices for Sampling Aggregates:

D 420 Recommended Practice for Investigating and Sampling Soil and Rock for Engineering Purposes ${ }^{3}$

D 653 Terminology Relating to Soil. Rock. and Contained Fluids ${ }^{3}$

D 1129 Definitions of Terms Relating to Water

D 1193 Specification for Reagent Water

D 2234 Method for Collection of a Gross Sample of Coals

D 2777 Practice for Determination of Precision and Bias of Methods of Committee D-19 on Water

D 3370 Practices for Sampling Water

This tes method is under the jurisdiction of ASTM Comminte D 34 on Waste Manggemen and is the direet responsbility of Subcommutre 134.03 on Physical and Chemical Characterization.

Current edition approved Sept. 22, 1992. Published November 1992

Antual Sook w.AST.M Stondards. Vol 04.03.

I. Annual Bout of.AST.M Siandards. Vol 04.08.

- Annual Book of.45T.1 Siandards. Vol 11.01.

${ }^{3}$ Annual Book of .AST.M Standards. Vol 05.05.

\section{D4793 Test Method for Sequential Batch Extraction Procedure}

\section{Terminology}

3.1 Definitions-For definitions of terms used in this test method. see Definitions D 1129.

3.2 Symbols - Variables listed in this test method are defined in the individual sections in which they are discussed. A list of the defined variables is also provided in Section 11.

\section{Significance and Use}

4.1 This test method is intended as a means for obtaining sequential extracts of a waste. The extracts may be used to estimate the release of certain constituents of the waste under the laboratory conditions described in this test method.

4.2 The $\mathrm{pH}$ of the extraction fluid used in this test method is to reflect the $\mathrm{pH}$ of acidic precipitation in the geographic region in which the waste being tested is to be disposed.

Vote 1-Possible sources of information concerning the $\mathrm{pH}$ of precipitation in the geographic region of interest include state and federal environmental agencies. suate universities libraries. etc.

Note 2-For sequential batch extraction of waste using a nonacidic extraction fuid, see Test Method D 4793.

4.3 An intent of this test method is for the final $\mathrm{pH}$ of each of the extracts to reflect the interaction of the extractant with the buffering capacity of the waste.

4.4 This test method is not intended to provide extracts that are represeniative of the actual leachate produced from a waste in the field or to produce extracts to be used as the sole basis of engineering design.

4.5 This test method has not been demonstrated to simulate actual disposal site leaching conditions.

4.6 This test method produces extracts that are amenable to the determination of both major and minor (trace) constituents. When minor constituents are being determined. it is especially important that precautions be taken in sample storage and handling to aroid possible contamination of the samples.

4.7 This test method has been tested to determine its applicability to certain inorganic components in the waste. This test method has not been tested for applicability to organic substances, volatile matter (see Note 5), or biologically active samples.

4.8 The agitation technique. rate. liquid-to-solid ratio, and filtration conditions specified in the procedure may not

\footnotetext{
- Innual Bur of ASTM Sundards. Vol 11.04.

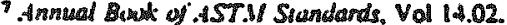


be suitable for extracting all types of wastes (see Sections 7 and 8 and Appendix XI).

\section{Apparatus}

5.1 Straight Edge. such as a thin-edged yardstick.

5.2 Impermeable Sheet, of glazed paper. oil cloth. or other nexible material of a composition suizable to the analytes of interest.

5.3 Drying Pans or Dishes (for example, aluminum tins. porcelain dishes. glass weighing pans). two per waste. suitable to the waste being tested and the instructions given in 9.2.

5.4 Drving Oven-Any thermostatically controlled drying oven capable of maintaining a steady temperature of $\pm 2{ }^{\circ} \mathrm{C}$ in a range of 100 to $110^{\circ} \mathrm{C}$.

5.5 Desiccator, having a capacity to hold the drying pans described in 5.3 and the crucibles described in 5.16.

5.6 Laboratory Balance. capable of weighing $100.1 \mathrm{~g}$.

5.7 Erlenmever Flask. 2.L capacity. equipped with a magnetic stir bar.

\subsection{Magnetic Stir Plate.}

5.9 Graduated Cylinder. 1 or $2-L$ capacity.

5.10 Pipet. $1 . \mathrm{mL}$ capacity.

5.11 Volumetric Flask. 1-L capacity.

5.12 Pipet. $10 \mathrm{~mL}$ capaciry. (Various other sized pipets. including micropipets. may be necessary for 9.3.2.)

$5.13 \mathrm{pH}$. Weter-Any $\mathrm{pH}$ meter with a readability of 0.01 units and an accuracy of \pm 0.05 units at $25^{\circ} \mathrm{C}$.

5.14 Carboy-1ype Container, with spigot. 20 to 50-L capacity, of a composition suitable to the nature of the analyses to be performed (see Practices D 3370).

5.15 Large Glass Funnel.

5.16 Crucibles. porcelain. 20-mL capacity each. two per waste.

5.17 Analvical Balance. capable of weighing $100.1 \mathrm{mg}$.

5.18 Wash Borle. 500-mL capacity.

3.19. Agitalion Equipment. of any ispe that rotates the

2-Liter Plastic or Glass Bottles
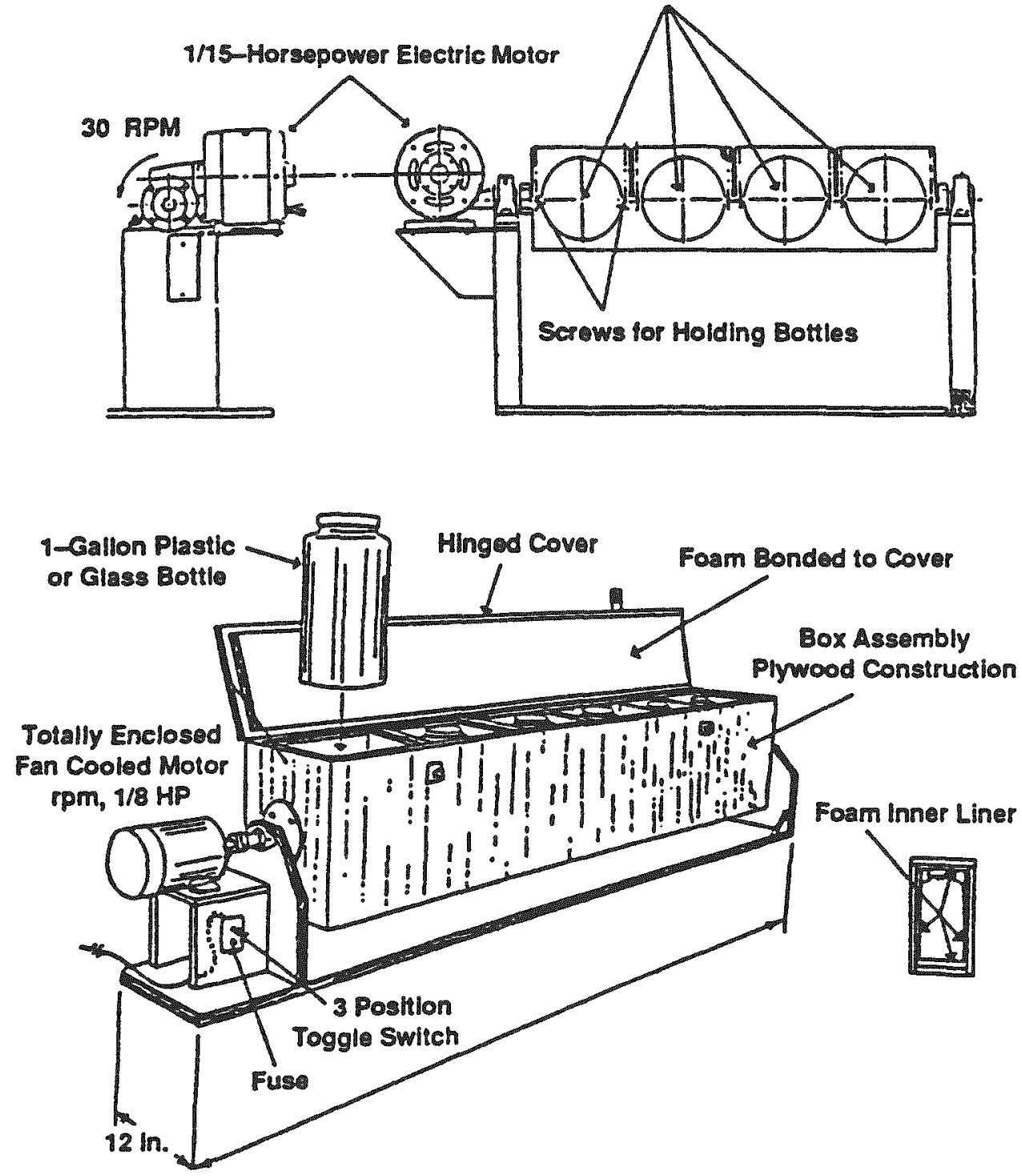

Fic. 1 Extractor 
extraction vessel in an end-over-end fashion at a rate of $30 \pm$ $2 \mathrm{r} / \mathrm{min}$ such that the axis of rotation is horizontal and it passes through the center of the bottle (see Fig. 1 and Appendix X1).

NoTE 3-Similar devices having a differen axial arrangement may be used if equivalency ean be demonstrated.

5.20 Pressure Filtration dssembly-A pressure filtration device of a composition suitable to the nature of the analyses to be performed and equipped with a 0.45 or $0.8-\mu \mathrm{m}$ pore size filter (see Viote 8 ).

5.21 Extraction Vessels, cylindrical. wide-mouth. of a composition suitable to the nature of the waste and analyses to be performed. constructed of materials that will not allow sorption of the constituents of interest. and sturdy enough to withstand the impact of the falling sample fragments. The size of the container should be selected so that the sample plus extraction fluid occupy approximately $95 \%$ of the container. The containers must have water-tight closures. Containers for samples in which gases may be released should be provided with venting mechanisms.

Note 4-Suirable container sizes range from 4.0 to $\$ .5$ in. in diamerer and 8.5 to $13.0 \mathrm{in}$. in height.

Note 5-The senung of the container has the potential to affect the concentration of volatile compounds in the exracts.

5.21.1 Extraction vessels should be cleaned in a manner consistent with the analyses to be performed (see Section 13 of Practice D 3370).

\section{Reagents}

6.1 Purity of Reagents-Reagent grade chemicals shall be used in all tests. Unless otherwise indicated. it is intended that all reagents shall conform to the specifications of the Commituee on Analvical Reagents of the American Chemical Society, where such specifications are available. ${ }^{8}$ Other grades may be used. provided it is first ascertained that the reagent is of sufficiently high purity to permit its use without lessening the accuracy of the determination.

6.2 Purity of "liter-Unless otherwise indicated. references to water shall be understood to mean Type IV reagent water at 18 to $27^{\circ} \mathrm{C}$ conforming to Specification D 1193. The method by which the water is prepared. that is. distillation, ion exchange, reverse osmosis, electrodialysis. or a combination thereof, should remain constant throughout testing.

6.3 Sulfuric Acid/Nitric Acid Solution-A 60/40 weight percent (wt \%) mixture prepared using 95 to 98 w \% sulfuric acid and 69 to 71 wi \% nitric acid. (See 9.3 for instructions on the preparation of this solution.)

\section{Sampling}

7.1 Obtain a representative sample of the waste to be tested by using. where available, ASTM sampling methods developed for the specific industry (see Practices D 75 and D 420. Terminology D 653. and Method D 2234).

7.2 Sarmpling methodology for materials of similar phys-

-Reanen Chernicals American Chemical Sociery Spectications."Am. Chemicat Soc. Washington. DC. For suggestuons on the testing of reagents not listed by the American Chernical Society. see "Reasent Chemicals and Sundards" by Joseph Rosin. D. Van Sustrand Co. Inc.. New Yort. VY. and the "United States Pharmacopera" ical form shall be used where no specific methods are available.

7.3 The amount of sample to be sent to the laboratory should be suficient to periorm the solids content determina. tion as specified in 9.2 . and to provide $100 \mathrm{~g}$ of sample on a dry weight basis for each extraction.

7.4 It is important that the sample of the waste be representative with respect to suriace area. as variations in surface area would directly affect the leaching characteristics of the sample. Waste samples should contain a representative distribution of paricle sizes.

VOTE 6-Information on obuining representative samples can also be found in Pierre G.'s Sampling Theory and Sampling Practuce."

7.5 In order to prevent sample coniamination or constiruent loss prior to extraction. keep the samples in closed containers appropriate to sample type and desired analysis. See Practices D 3370 for guidance. Record the storage conditions and handling procedures in the report.

7.6 The time between collection and extraction of the sample should be determined by the nature of the sample and the information desired. See Practices D 3370 for guidance. Report the length of time between sample collection and extraction.

\section{Sample Preparation}

8.1 For free-flowing particulate solid wastes. obtain a sample of the approximate size required in the test by quartering the sample (Section 7) received for testing on an impermeable sheet of glazed paper. oil cloth, or other flexible material having composition suitable to the analytes of interest. as follows:

8.1.1 Empty the sample container into the center of the sheet.

8.1.2 Genty flatten the sample out with a suitable straightedge until it is spread uniformly to a depth as least twice the maximum particle diameter.

8.1.3 Remix the sample by lifing a comer of the sheet and drawing it low across to the opposite comer in such a manner that the material is made to roll over and over and does not merely slide along. Continue the operation with each comer. proceeding in a clockwise direction. Repeat this operation ten times.

8.1.4 Lift all four comers of the sheet toward the center and. holding all four comers together. raise the entire sheet into the air to form acket for the sample.

8.1.5 Repeat the procedure described in 8.1.2 to natren the sample out.

8.1.6 With a straightedge (such as a thin-edged yardstick) at least as long as the flattened mound of sample. gently divide the sample into quarters. Make an effort to avoid using pressure on the straightedge sufficient to cause damage to the particles.

8.1.7 Discard the alternate quarters.

8.1.8 If further reduction of the sample size is necessary, repeat the steps given in 8.1.3 through 8.1.7. Use a sample size to provide $100 \mathrm{~g}$ of solid on a dry weight basis for each extraction. Provide additional samples for the determination

\footnotetext{
- Picard. F.. Pierre G.'s Samplung Theori and Sampleng Practuce. Vols i and It
} CRC Press 1989. 
of solids conteni (see 9.2). Use of a sample size other than $100 \mathrm{~g}$ of solid on a dry weight basis for extraction is not recommended: however. if a differen sample size is used. repon this fact.

Note 7 -For other acceptable methods of mixing and subsampling freeflowing solid pariculate wastes. see Pierne Gy's Sumping Theory and Sampling Practice." The method of subsampling should be deter. mined by the physical properties of the waste. analyes of interest. and equipment available.

8.2 For field-cored solid wastes or castings produced in the laboratory, cut a representative section weighing approximately $100 \mathrm{~g}$ for testing. plus samples for the determination of solids content. Shape the sample so that the leaching solution will cover the material to be leached.

8.3 For multiphasic wastes. mix thoroughly to ensure that a representative sample will be withdrawn. Take samples for the determination of solids content at the same time that test samples are taken.

\section{Procedure}

9.1 Record a physical description of the sample to be tested. including particle size so far as it is known.

9.2 Solids Content-Determine the solids content of two separate porions of the sample as follows:

9.2.1 Dry to a constant weight. at $104 \pm 2^{\circ} \mathrm{C}$. two dishes or pans of size suitable to the solid waste being tested. Cool in a desiccator and weigh. Record the values to $\pm 0.1 \mathrm{~g}$.

9.2.2 Place $50 \mathrm{~g}$ of the waste to be tested into each pan. Record the mass of sample in each pan to $\pm 0.1 \mathrm{~g}$.

9.2.3 Dry 16 to $20 \mathrm{~h}$ at $104 \pm 2^{\circ} \mathrm{C}$. Record the temperature and time of the drying period.

9.2.4 Cool to room temperature in a desiccator and reweigh. Record the mass to $\pm 0.1 \mathrm{~g}$.

9.2.5 Repeat the steps given in 9.2.3 and 9.2.4 until constant container-sample masses are obtained. Discard the dried samples following completion of this siep.

9.2.6 Calculate the solids content of the sample from the data obtained in 9.2.1. 9.2.2. and 9.2.7 as follows:

$$
S=A / B
$$

where:

$A=$ mass of sample after drying. g.

$B=$ original mass of sample, g. and

$S=$ solids content. $\mathrm{g} / \mathrm{g}$.

Average the two values obtained. Record the solids content.

9.3 Preparation of Extraction Fluid-Prepare a $60 / 40$ wt \% mixture of sulfuric acid/nitric acid. Cautiously mix 60 $\mathrm{g}$ of concentrated sulfuric acid with $40 \mathrm{~g}$ of concentrated nitric acid. The preparation of this mixture should be performed in a laboratory fume hood.

9.3.1 Using the $60 / 40^{\circ}$ sulfuric acid/nitric acid mixture, prepare a second solution by diluting $1.0 \mathrm{~mL}$ of the $60 / 40$ mixture to $1000 \mathrm{~mL}$ using water and a 1-L volumetric flask.

9.3.2 Using the $1 / 1000$ solution prepared in 9.3.1. prepare the extraction fluid having the desired $\mathrm{pH} \pm 0.05$ (see 4.2 ) by pipeting a volume of the $1 / 1000$ solution into $2000 \mathrm{~mL}$ of water with mixing until the desired $\mathrm{pH} \pm 0.05$ is achieved. A recommended method for preparing the extraction thid is to add $2000 \mathrm{~mL}$ of water to a 2-L erlenmever flask equipped with a magnetic stir bar. Place the erlenmeyer flask on a magnetic stir plate. and add the $1 / 1000$ solution to the flask with stirring. Shake the mixture vigorously. and measure its $\mathrm{pH}$ once the solution is static. Continue this process until the desired solution $\mathrm{pH} \pm 0.05$ is reached. Record the amount of $1 / 1000$ solution added to $2000 \mathrm{~mL}$ of water to achieve the desired $\mathrm{pH} \pm 0.05$. Record the $\mathrm{pH}$ value of the solution. Additional 2-L batches of the extraction nuid can be prepared by mixing the determined volume of $1 / 1000$ solution with $2000 \mathrm{~mL}$ of water. The $\mathrm{pH}$ of the extraction fluid must be within \pm 0.05 of the desired value for use in the extraction procedure. For extracting different wastes requiring the same extraction fuid pH or performing replicate extractions. multiple batches of extraction fluid can be prepared and measured for correct $\mathrm{pH}$, and if the $\mathrm{pH}$ is within $₫ 0.05$ of the desired value. the batches can be combined in a carboy-lype coniainer of a composition suitable to the nature of the analyses to be performed. The $\mathrm{pH}$ of the resulting solution in the carboy must be measured once again to verify the correct $\mathrm{pH}$ before using the solution in the extraction procedure and as a rinse solution (see the procedures given in 9.4 and 9.7). If the $\mathrm{pH}$ value is not swithin the above specification. the solution shall not be used. and fresh extraction fluid shall be prepared. Record the $\mathrm{pH}$ value of each batch. and of the solution in the carboy. prior 10 its use.

9.4 Exrraction Procedure-If the entire procedure cannot be conducted without interruption. at least the first four extraction sequences must be conducted without intemution.

9.4.1 Determine the mass of the extraction vessel to be used in the extraction procedure to the nearest $0.1 \mathrm{~g}$. Record the mass of the extraction vessel. $M_{\mathrm{r} / \mathrm{.}}$. Use one extraction vessel per waste throughout the sequence of extractions.

9.4.2 Add $100 \mathrm{~g}$ (weighed to $\pm 0.1 \mathrm{~g}$ ) of solid waste on a dry weight basis to the extraction vessel. Calculate the amount of as-received waste to add using the following equation:

$$
y=\frac{100}{5}
$$

where:

$S=$ solids content $(\mathrm{g} / \mathrm{g})$ determined in 9.2.6. and

$M=$ mass of as-received waste (weighed to $\pm 0.1 \mathrm{~g}$ ) to add to the extraction vessel to vield $100 \mathrm{~g}$ of solid waste.

9.4.2.1 If a mass of solid waste on a dry weight basis other than $100 \mathrm{~g}$ is used. Eqs (2) through (4) must be modified to retlect the use of a mass other than $100 \mathrm{~g}$. Replace 100 in these equations with the mass used. The use of a mass other than $100 \mathrm{~g}$ is not recommended.

9.4.3 Add a mass in $g . M_{\mathrm{cm}}$ of extraction fluid (see 9.3) to the extraction vessel determined using the following equations:

where:

$$
H_{s w}=M-100
$$

$M_{s w}=$ mass of moisture $(\mathrm{g})$ in the sample added to the extraction vessel. and

$$
U_{d f}=(20)(100)-H_{\text {sw }}
$$

This uill provide a solid-to-liquid ratio of $1: 20$ in the extraction vessel.

9.4.4 Agitate continuousiy for $18 \pm 0.35 \mathrm{~h}$ at 18 to $27^{\circ} \mathrm{C}$. Record the agization time and temperature. 
9.4.5 Open the extraction vessel. Observe and record any -visible physical changes in the sample and leaching solution. Record the $\mathrm{pH}$ of the waste/leaching solution slurry.

9.5 Filtration-Transfer as much of the waste/leaching solution as possible through a large glass funnel to a pressure filtration device equipped with a 0.45 or $0.8 \mu \mathrm{m}$ filter. Transfer the mixed slurry. Do not decant. Invert the extraction vessel over the filtration device and allow the liquid to drain for $1 \mathrm{~min}$ from the solid remaining in the extraction vessel. It is important 10 achieve as complete a transfer of fluid from the extraction vessel to the filtration device as possible. Pressure tilter the liquid through the filter using nitrogen gas or an iner gas that will not contaminate or change the integrity of the sample. After the extract has passed through the filter. continue running gas through the filtration device at 30 psi for $3 \mathrm{~min}$. The filtrate obtained is the extract mentioned in this test method (see 9.6 and 10.8). Determine the mass of the filtrate collected and repor it as $M_{f}$ for the extraction step. Measure the $\mathrm{pH}$ of the extract immediately. remove the amount of filtrate necessary lor the determination of total dissolved solids content in 9.6. and then preserve the extract in manner consistent with the chemical analyses or biological testing procedures to be performed (Practices D 3370. Section 15).

Nore 8-Analtrical results may be attected by the type of fitrer used. If a $0.8-\mu \mathrm{m}$ tilter pore size is used. the resulting extract should be digested prior to elemental analysis. The composituon of the filter should also be considered. If the filter is composed of material that may contaminate the extract during tiltration. the filter should be washed in the filtration device in a manner consistent with the chemical analyses or biological sestung procedures to be periormed on the extract. For example. for elemental analysis of the extract. if a titter composed of borosilicate glass fiber is used. in order to preven! contamunation. it should be washed in the filration device uith a dilute acid solutuon and rinsed with approximately $2 \mathrm{~L}$ of water prior to tiltration.

Note 9-Prefilters can be used only if it is absolutely necessary (filtrate for analysis or testing sannot be obtained unless a presitter is used) due to the loss of sample trapped in the pores of the pretilter and the possibility of the prefiher disintegraung during ansing.

Note $10-$ it is recommended that all tiltrawons be performed in a hood.

9.6 Tolal Dissolved Solids Comen ITDS/-Add a 10.0-g aliquot of the extract to each of two $110^{\circ} \mathrm{C} \pm 2^{\circ} \mathrm{C}$ dried. preweighed crucibles. Place the samples in a drving oven as $110^{\circ} \mathrm{C} \pm 2{ }^{\circ} \mathrm{C}$ for $3 \mathrm{~h}$. Record the dring oven temperature and drying sime. Remove the crucibles and let them $\mathrm{cool}$ in a desiccator. Reweigh the crucibles and record their weights to $\pm 0.1 \mathrm{mg}$.

NoTE $11-$ Only one drying is performed to limit the contact time between the solid and the rinse solution in the extration vessel prior to the nexi exiraction step (see 9.7. Section 10. and 10.7).

9.6.1 If the mass of solid lost through dissolution..$L_{d}$ (see 10.2). in the first extract is less than 196 of the mass of solid used in the first extraction step. and if the percent of solid lost through dissolution in the second extrastion step is less than or equal to the percent of solid lost through dissolution in the first extraction step. the determination of TDS in the following extracts is not required. and the user can assume that TDS and $M_{d}$ are equal to 0 for Eqs $(6)$ and (7) for Extracts 3 through 10.

9.7 Quantitatively transfer the damp solid from the filter back to the original extraction vessel. including the filter. Use extraction huid. prepared as described in 9.3. from a preweighed wash bottle to assist in this transfer and to nnse the filtration device. No more than 500 of rinse solution should be used. Use the smallest amount of rinse solution possible to achieve a thorough transfer. Using tweezers or a similar device. recover the filter and rinse the adhering solid into the extraction vessel with rinse extraction fluid from the pre-weighed wash bottle. Do not leave the filter in the extraction vessel. Reweigh the wash bottle to determine the amount of rinse solution used in the transfer. Record this value as $\|_{\mathrm{F}}$. Weigh the extraction vessel following the transfer described above. and record this value as $M_{r}$. The extraction vessel may be sealed until a feasible time for the addition of new extraction fluid. This is to enable filtration during the next sequence at a reasonable time during the day. If the slurry is stored for longer than $6 \mathrm{~h}$ in the extraction vessel prior to the addition of new extraction fluid. the data generated by analysis of the extracts should be plotted to check for perturbation of the data curve.

\section{Calculation}

10.1 Calculate the TDS. in milligrams per gram of the filtrate. using the following equation:

$$
\operatorname{TDS}=\left(.1 / t_{\mathrm{sc}}-.1 \mathrm{gs}_{\mathrm{s}}\right) /(10 \mathrm{~g})
$$

where:

$. M_{\mathrm{st}}=$ mass of the crucible and dried solids. mg. and

$M_{c}=$ mass of the crucible. $\mathrm{mg}$.

10.2 Calculate the mass of the solid. in grams. lost through dissolution..$/ /_{s}$ using the following equation:

$$
\left.H_{d}=\operatorname{TDS}\right)\left(. H_{f}\right)(0.001)
$$

where:

$u_{f}=$ mass of filtrate collected in that extraction. g. and

$\|_{t}=$ mass lost through dissolution.

10.3 Calculate the mass of the solid. in grams. corrected for TDS remaining for the next extraction step. $H_{s}$. using the following equation:

$$
M_{\mathrm{s}}=. M_{s}-1-H_{d}
$$

where:

$. b_{s}{ }^{2-1}=$ mass of the solid extracted in the current extraction step. g.

: vote 12-For example. in beginning the first extraction. $1 \mathrm{I}_{\mathrm{f}}^{-1}$ will equal $100 \mathrm{~g}$ and to calculate the mass of solid remaining for the second

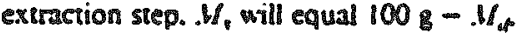

10.4 Calculate the combined mass of the solid and the residual liquid in the extraction vessel. M $_{\mathrm{s}}$, using the following equation:

$$
M_{s t}=M_{r}-M_{s f}-M_{R}
$$

10.5 Calculate the mass of liquid adhering to the solids in the extraction vessel. $4 \%$. using the following equation:

$$
I_{l}=. H_{s l}-. I_{s}
$$

10.6 Calculate the mass, in grams. of extraction fluid to be added to the extraction vessel. extraction fluid mass (EFM). using the following equation:

$$
\mathrm{EFM}=\left[\left(H_{s}\right)(20)\right]-. H_{l}-H_{n}
$$

10.7 Add the amount of extraction fluid. EFM. determined in 10.6 to the extraction vessel and repeat the 
procedures described in 9.4 .3 through 10.7 so that ten - extractions are conducted in sequence.

Vore 13-This procedure assumes that the amount of waste trapped in the filters after nusing is negligible.

10.8 Analyze the extracts for specific constiuents or properties. or use the extracts for hiological testing procedures as desired. using appropriate ASTM test methods. Where no appropriate ASTM test methods exist. other test methods may be used and recorded in the repor. Whether visible phase separation during storage of the exiracis occurs or not. appropriate mixing should be used to ensure the homogeneity of the extracts prior to their use in such analyses or testing.

10.9 Compensation for Curry:O1er-For each constituent in each of the extracts generated in the extraction sequence. the contribution to concentration from the residual liquid from the previous extraction step. $C_{p}$ can be calculated using the following equation:

$$
\left.C_{i}=\left[M / 1 / 201 M^{a-1}\right]\right]\left[C_{3}\right]
$$

where:

$C_{8}=$ concentration of the constituent in the filtrate from the previous extraction step.

$M_{h}=. M_{\text {f }}$ from the previous exiracion siep. and

$\|_{s}{ }^{-1}=$ mass of solid extracted in the current extraction step (see Note 12).

\section{Definitions of Variables}

11.1 The following variables must be determined when performing the sequential batch extraction procedure:

11.1.1 Solids Cuntent Determinatun:

.1 = mass of sample after drying in the determination of solids content of the waste to be extracied.

$B=$ original mass of the sample prior to dring in the determination of solids content of the waste to be exiracted. g. and

$S=$ solids content of the waste to be extracted. $g / g$.

11.1.2 First Evtraction Step:

$M=$ mass of as-received waste added to the extraction vessel to vield $100 \mathrm{~g}$ (weighed to $\pm 0.1 \mathrm{~g}$ ) of solid on a dry weight basis for the first extraction step. g.

$M_{a}=$ mass of extraction fuid to be added for the first step in the extraction procedure. $g$. and

$M_{s 1}=$ mass of moisture in the sample to be extracted in the first exiraction step. $\mathrm{g}$.

11.1.3 TDS Determinatuon:

TDS = total dissolved solids content of the filtrate. $\mathrm{mg} / \mathrm{g}$.

$M$. = mass of the crucible to be used in the TDS determination. mg. and

$M_{s}=$ mass of the crucible and dried solids in the TDS determination. mg.

11.1.4 Extraction Sequence:

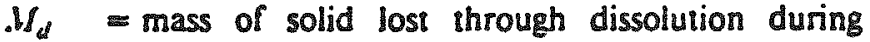
extraction. 8.

.1. = mass of hitrate collected in that extraction. $g$.

$M_{s}=$ mass of solid remaining for the nexi extraction step. g.

$M_{s}{ }^{-1}=$ mass of solid extracied in the current extraction step. g.

$. I_{y}=$ mass of the empty extraction vessel. $g$.

${ }_{{ }}{ }_{R}=$ mass of the rinse solution. $g$.
. solution. solid and moisture in the solid. and solid and liquid left in the extraction vessel atter transter to the liltering device. $g$.

$. I_{v}=$ combined mass of solid and residual liquid in the extraction vessel following transter of the moist sample cake back to the extraction vessel, $g$.

. ion vessel following ranster of the moist sample cake back to the extraction ressel. g. and

EFM = mass of extraction lluid to be added for the nexi extraction step. $g$.

11.1.5 Compensation ior Carri-Over:

$C_{f}=$ contribution io a constituent's concentration in the curren step from the residual liquid of the previous extraction step. mg/L.

$M_{f_{1}}=M_{1}$ from the previous extraction step. $g$.

$M^{s-1}=$ mass of solid extracted in the current step. $g$. and

$C_{1}=$ concentration of constituent in the filtrate from the previous extracion step. $\mathrm{mg} / \mathrm{L}$.

\section{Report}

12.1 Repon the following intormation:

12.1.1 Source of information concerning the $\mathrm{pH}$ value of the precipiation in the geographic region of interest (see Note 1):

12.1.2 Source of the waste. date of sampling, methods of sampling and sample preservition. storage conditions, han. dling procedures. and length of ime between sample collection and extraction:

12.1.3 Description of the waste. including its physical characteristics and particle size. if known (9.1):

12.1.4 Solids content (9.2):

12.1.5 Mass of solid uaste on a dri weight basis extracied. if other than $100 \mathrm{~g}(8.1 .8)$ :

12.1.6 pH of the extraction huid used for each extraction sequence. and as wash solution tor each transfer:

12.1.7 Time and temperature used in the determination of solids content and TDS:

12.1.8 Agitation temperature and time:

12.1.9 Filter pore size used and filter composition:

12.1.10 Use of a prefilier. pretilier pore size. and composition:

:2.1.11 Observations of changes in the test material or leaching solution (9.4.4):

12.1.12 Storage of the solid with rinse solution in the exiraction vessel for any period longer than $6 \mathrm{~h}$ :

$12.1 .13 \mathrm{pH}$ before and after intration. and the results of specific analyses calculated in appropriate units and correcied for carry-over. if necessan: and

12.1.14 Dates on which sequential batch extraction was stared and completed. presenation used for extracts. and dates of analyses.

Note 14-Fig 2 presents a summary report format for recording some of the experimental uata. and Fig. 3 is a detailed iaboratory workshet that may be helplul in periorming the test method.

\section{Precision and Bias}

13.1 Precision-Data on the precision of this test method will be generated in an upcoming collaborative study of the test method. Practice D 2777 will be used to evaluate the data statistically. 


\section{(7) D 5284}

Sample Number:

\begin{tabular}{|c|c|c|c|c|c|c|c|c|c|c|}
\hline $\begin{array}{l}\text { In Generaung } \\
\text { Extraet Number }\end{array}$ & $\begin{array}{c}\text { TOS } \\
\text { (mg/g) }\end{array}$ & $\begin{array}{l}M_{0} \\
(g)\end{array}$ & $\begin{array}{l}M \\
(g)\end{array}$ & $\begin{array}{l}M_{3} \\
(g)\end{array}$ & $\begin{array}{l}M_{0} \\
(g)\end{array}$ & $\begin{array}{l}M \\
(g)\end{array}$ & $M_{(g)}$ & $M_{a}$ & $\begin{array}{l}M \\
\text { (g) }\end{array}$ & $\begin{array}{l}\text { EFM } \\
(g)\end{array}$ \\
\hline \multicolumn{11}{|l|}{1} \\
\hline \multicolumn{11}{|l|}{2} \\
\hline \multicolumn{11}{|l|}{3} \\
\hline \multicolumn{11}{|l|}{4} \\
\hline \multicolumn{11}{|l|}{5} \\
\hline \multicolumn{11}{|l|}{6} \\
\hline \multicolumn{11}{|l|}{7} \\
\hline \multicolumn{11}{|l|}{8} \\
\hline \multicolumn{11}{|l|}{9} \\
\hline 10 & & & & & & & & & & \\
\hline
\end{tabular}

FiG. 2 Sequential Iatch Procedure Data Sheet

13.2 Bias-Determination of the bias of this test method is not possible. as no standard reference material exists. Information concerning analytical bias will be obtained from the analysis of standard solutions in the upcoming collaborative study on the test method.

\section{Keywords}

14.1 acidic precipitation: extract: extraction nuid: leaching: sequential batch extraction: waste leaching technique 
Sample:

Sohds Content Determinaton:

Dish 1 mass after dryng:

Dish 2 mass atter drying:

Sample i mass belore dryng (B I)

Sample 2 mass belore dryng (8. 2 ):

Drying temperature:

Drying wne:

Sample I mass after oryung (A I):

Sample 2 mass after oryng (A 2):

Solids Content 1 ( $S$ 1): $S=A=1 / B=$

Solids Content 2 (S*2): $S=A=2 / B=$

Average Solids Content (S) =

\section{Extraction : \\ Mass of emply extraction vessel $\left(M_{4}\right)$ :}

Mass of sample to be extracted (M): $M=100 / 5=$

Mass of moisture in the sample to be extracted $\left(M_{3 m:}: M_{9 w}=M-100=\right.$

Mass of extraction fiud to be added $\left(M_{e n}\right): M_{10}=(20)(100)-M_{w}=$

pH of extrecton flud:

Extraction une and temperature:

Filter type and pore-size:

Mass of fitrate collected $\left(M_{1}\right)$ :

pH of extract:

Mass of wash botule before transier:

Mass of extraction vassel after solud transter back $\left(M_{v}\right)$ :

Mass of wash botile atter iransfer:

Mass of rinse solution used in transfer $\left(M_{M}\right)$ :

Total Oissolved Solids Content of Extract 1

Crucible 1 mass $\left(M_{c} 1\right)$ :

Crucible 2 mass $\left(M_{c}\right.$ \%):

Subsample 1 mass:

Subsample 2 mass:

Dryng remperature:

Drying ime:

Crucble 1 phus residue mass $\left(M_{2 e}\right.$ \#1):

Crucible 2 plus residue mass $\left(M_{2 c}\right.$ :2):

Total Dissolved Solids Content (TOS):

TOS $1=\left(M_{\mathrm{zg}}+1-M_{\mathrm{c}}+1\right) /(10 \mathrm{~g})$

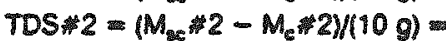

Average TOS value:

Mass of solid lost through dissolvton $\left(M_{a}\right): M_{a}=(T D S)\left(M_{1}\right)(0.001)$

$$
M_{\infty}=
$$

Mass of solid remanung for second extracton step $\left(M_{2}\right): M_{g}=100-M_{a}$

$$
M_{3}=
$$

Mass of solid and residual iqud in the extraction vessel $\left(M_{M}\right)$ :

$M_{s}=M_{v}-M_{v}-M_{A}$

$M=$

Mass of liquid adhenng to solids in the extraction vessel (MJ:

$M_{i}=M_{2}-M_{2}$

$M_{1}=$

Mass of extraction fluid to add for the second extraction step (EFM):

$E F M\left(M_{0}\right)(20)-M_{1}-M_{\text {F }}$

EFM

FIC. 3 Sequential Batch Extraction Laboratory Wortshest 
Sequential Extraction Labortory Worthel-Continued

Sample:

Extraction $(2-10)$

PM of extraction finud:

Extraction ume and temperature:

Filter type and pore-size:

Mass of fitrate contected (M):

PM of extract:

Mass of wash bort before ipansfer:

Mass of extraction vessel ather solvd transter back $\left(M_{v}\right)$ :

Mass of wash botrle after transter:

Mass of rinse solution used in iransfer $\left(M_{\text {a }}\right)$ :

Total Dissolved Solids Content of Extract (if necessary)

Crucible 1 mass $\left(M_{c} 1\right)$ :

Crucible 2 mass $\left(M_{e}\right.$ 赫):

Subsample 1 mass:

Subsample \#2 mass:

Orying remperature:

Dryng tume:

Cruable 1 plus residue mass $\left(M_{2 c}\right.$ 1):

Crucible 2 plus residue mass $\left(M_{24}\right.$ 2):

Total Dissolved Solids Content (TDS):

TOS产 $1=\left(M_{2 e} 1-M_{c}\right.$

TDS $2=\left(M_{s c}=2-M_{E} \$ 2\right)(10 g)=$

Average TOS valuo

Mass of solid losi through dissolution $\left(M_{d}\right): M_{x}=(T D S)\left(M_{q}\right)(0.001)$

$$
M_{a}=
$$

Mass of solid remaining for next extraction step $\left(M_{2}\right): M_{5}=M_{8}^{e-1}-M_{0}$

$$
\mathrm{M}_{3}=
$$

Mass of solid and residual liquid in the extraction vesset ( $M_{24}$ ):

$$
\begin{aligned}
& M_{s t}=M_{v}-M_{w}-M_{9} \\
& M_{g a}=
\end{aligned}
$$

Mass of liquid achenng to solds in the extraction vessel (M):

$$
\begin{aligned}
& M_{1}=M_{s s}-M_{s} \\
& M_{1} \equiv
\end{aligned}
$$

Mass of extrecton fluid to add for the next extractron step (EFM):

$$
E F M=\left(M_{2}\right)(20)-M_{1}-M_{\text {M }}
$$

$E F M=$

FIG. 3 Continus 


\section{APPENDIX}

\section{(Nonmandatory Information)}

\section{I1. AGITATION TECHNIQLES AND RATE AND LIQLID/SOLID RATIO}

XI.I The agitation rate. equipment. and liquid/solid ratio specified in this test method may influence the results on cerain solid wastes significantly. and it may not be adequate for certain solid wastes. such as monolithic. solidified. or organic wastes.

X1.2 The possible effects of varving the agitation techniques and rate include degree of mixing. rate of release of constituents. and particle abrasion effects. The precision of this test method may also be inlluenced.

X1.3 The possible effects of varving the liquid/solid ratio include degree of mixing. rate of release of constituents land possible concentration effects. depending on availability). and particle abrasion effects.

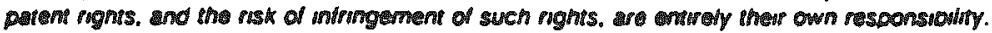

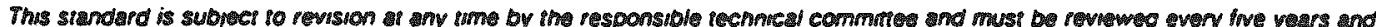

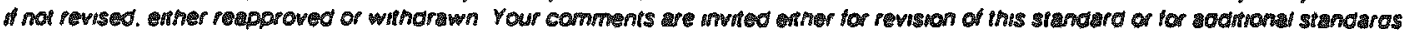

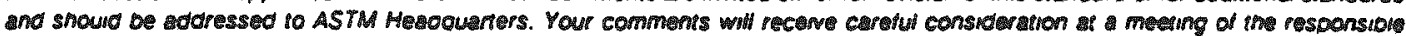

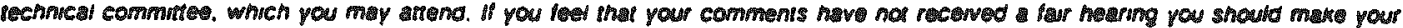
Ww's known to the ASTM Commme on Sincarcs. 1916 Rece St. Phutwetphis, PA 19103. 
APPENDIX $G$

Precision and Bias Statements to be Added to ASTM Method D5284-92 


\section{Precision and Bias ${ }^{10}$}

\subsection{Precision:}

13.1.1 A collaborative study of this test method involving ten laboratories was conducted. A spray dryer waste from an innovative clean coal technology process and a composite mining waste were extracted ten times in duplicate using this test procedure. The spray dryer waste was extracted using an extraction fluid having a $\mathrm{pH}$ of $4.3 \pm 0.05$, and the composite mining waste was extracted using an extraction fluid having a pH of $5.0 \pm 0.05$. Nitrocellulose filters having a pore size of $0.45-\mu \mathrm{m}$ were used by the collaborative study participants for the filtering specified in section 9.5 . The resulting extracts were then analyzed for specific inorganic analytes. The collaborative study participants analyzed six analytical standards in triplicate to generate data for calculating analytical precision. These standards contained high, medium, and low concentrations of the elements of interest in the extracts. In addition, eight special samples were analyzed by the laboratories to generate data for evaluating the effects of filter pore size, and digestion versus nondigestion, on analytical values determined in the extracts. To prepare the special samples, extractions of four samples each of the spray dryer waste and the composite mining waste were performed using the first extraction step. Two extracts from each waste were generated by filtering the extraction slurries through $0.45-\mu \mathrm{m}$ pore-size filters, and two extracts from each waste were generated by filtering the extraction slurries through $0.8-\mu \mathrm{m}$ pore-size filters. One of the $0.45-\mu \mathrm{m}$ extracts and one of the $0.8-\mu \mathrm{m}$ extracts from each waste were digested using U.S. Environmental Protection Agency (EPA) Method 3010.11 Portions of the 0.8- $\mu \mathrm{m}$ and the $0.45-\mu \mathrm{m}$ filtrates and digestates were sent to the collaborative study participants for analysis. All of the participants in the study analyzed the regular extracts, analytical standards, and special analytical samples using inductively coupled plasma spectrometry. Two of the laboratories digested their extracts and analytical standards prior to analysis. The other participants did not digest the extracts and standards. Information on the specific methods used by the participants is available in Report PCN:3313.1.2 In the collaborative study, some of the laboratories teamed together so that eight data sets were generated by the participants. Two of the laboratories did not follow the study specifications, and as a result, six data sets were used to evaluate the precision of the extraction procedure. ASTM Practice D2777 was used as a guideline for the statistical evaluation of the data.

${ }^{10}$ Supporting data for the precision and bias information are available from ASTM Customer Service and may be obtained by requesting Report PCN:33-00000456.

${ }^{11}$ U.S. EPA, 1990, Method 3010: Acid Digestion of Aqueous Samples and Extracts for Total Metals for Analysis by Flame Atomic Absorption spectroscopy or Inductively Coupled Plasma spectroscopy. Test Methods for Evaluating Solid Waste: Physical/Chemical Methods (SW846), Vol, 1A, 3rd Ed. 
13.1.3 The data generated in this collaborative study are specific to the test materials used in the study, the elements of interest, the pH values of the extraction fluids used, and $0.45-\mu \mathrm{m}$ filter pore-size filtration. For other materials, elements, pH values, and filter types, these data may not apply.

13.1.4 The data generated in the collaborative study can be divided into three categories: (1) data from extraction of the test materials and analysis of the resulting extracts, which were used to determine the mean concentration for each element in each extract, $\overline{\mathrm{z}}$, and the total standard deviation of the extraction procedure-plus-analysis of the extracts, $s ;(2)$ data from analysis of the analytical standards, which were used to determine the mean concentration for each level of analytical standard, $\bar{x}_{a}$, and the standard deviation for analysis of the analytical standards, $\mathbf{s}_{\mathrm{a}} ;$ and $(3)$ data from analysis of the special analytical samples, which were used to calculate the mean concentration for each type of special sample and the standard deviation to evaluate the effects of filter pore size, and digestion versus nondigestion, on the analytical concentrations in the extracts.

13.1.5 Three types of precision can be determined from the data generated in the collaborative study. These are: the total standard deviation, s, which is described in section 13.1 .4 ; the analytical standard deviation, $s_{2}$, which is also described in section 13.1.4; and the estimated standard deviation of the extraction procedure, $s_{e}$, which represents the estimated error due to only the extraction method. The estimated multiple-laboratory standard deviation of the extraction procedure, $s_{e}$, for each element of interest in the two test materials was calculated using the equation:

$$
s_{e}=\left[s^{2}-s_{a}^{2}\right]^{1 / 2} \text {, }
$$

where $\mathbf{s}$ is the standard deviation of the extraction procedure-plus-analysis of the extract, and $s_{a}$ is the standard deviation for analysis of the analytical standard containing the concentration of the specific element closest to its concentration in the extract. These estimated multiplelaboratory values for the elements of interest in extracts 1, 3, 5, 7, and 10 of the spray dryer waste and composite mining waste, along with the mean concentration values, $\bar{x}$ and $\bar{x}_{a}$, are listed in Tables 1 and 2 . These data, for all ten of the extracts of the spray dryer waste and composite mining waste, are available in Report PCN:33-000004-56.

13.1.6 The three types of precision values discussed in sections 13.1 .4 and 13.1 .5 , total, analytical, and extraction procedure, can also be calculated based on a single operator. Calculations were performed to determine the total single-operator precision, so, the single-operator analytical standard deviation, $s_{o a}$, and the estimated single-operator precision of the extraction procedure, $s_{o e}$. The estimated single-operator precision of the extraction procedure was calculated using the equation:

$$
s_{o e}=\left[s_{0}^{2}-s_{o a}^{2}\right]^{1 / 2},
$$

where $s_{0}$ is the single-operator standard deviation of the extraction procedure-plus-analysis of the extract, and $s_{\text {oa }}$ is the single-operator standard deviation for analysis of the analytical standard containing the concentration of the specific element closest to its concentration in the extract. The estimated single-operator precisions of the extraction procedure for the elements of interest in extracts 1, 3, 5, 7, and 10 of the spray dryer waste and composite mining waste are listed in Tables 3 and 4. 
These values, for all ten of the extracts of the spray dryer waste and composite mining waste, are available in Report $\mathrm{PCN}: 33-000004-56$.

13.1.7 It was not economically practical to determine analytical precision using actual extracts of the wastes because of the extensive number of analyses that would have been required. The analytical standards were, therefore, used to determine analytical precision. Calculation of the standard deviation of the extraction procedure can provide only an approximation because the analytical standards do not contain the specific matrix resulting from the interaction of the extraction fluid and solid waste. In addition, the analytical standards are limited to three concentration levels for each element. To calculate the precision of the extraction procedure for a particular element, the analytical standard deviation for analysis of the analytical standard containing the concentration of the element closest to its concentration in the extract was used. For some of the extracts, the elemental concentration in the extract varies significantly from the element's closest concentration in the analytical standards. Because of the way in which the total precision and analytical precision were determined, in some cases, the analytical standard deviation values, $s_{a}$ and $s_{o a}$, are larger than the total standard deviation values, $s$ and $s_{0}$. In these cases, the precision of the extraction procedure can not be determined.

13.1.8 The estimated precision of this sequential batch extraction procedure varies somewhat with the concentration of aluminum, barium, silicon, sodium, and strontium in the spray dryer waste extracts according to Figures 1 to 5 , respectively, and the estimated precision of the sequential batch extraction procedure varies somewhat with the concentration of calcium, magnesium, manganese, and silicon in the composite mining waste extracts according to Figures 6 to 9 , respectively. Figures 1 to 9 are plots of the calculated percent relative standard deviation of the extraction method versus the mean concentration of the constituent in the extract. The threedimensional bar graphs are helpful in examining trends and interpreting the data. As expected, the relative precisions of the single-operator values are better than those of the multiple-operator values, and the single-operator relative precisions are encouragingly good. Plots for the other elements of interest in the spray dryer waste and composite mining waste can be found in Report PCN:33-000004-56.

13.1.9 Collaborative study data from analysis of the special analytical samples show that filter pore size, $0.45-\mu \mathrm{m}$ versus $0.8-\mu \mathrm{m}$, and digestion versus nondigestion, affect certain elemental concentrations determined in the extracts of the spray dryer waste and composite mining waste. The data also show that these effects are waste and element specific. Data from analysis of the special analytical samples are available in Report PCN:33$000004-56$.

13,2 Bias-Determination of the extraction bias for this test method is not possible, as no standard reference material exists. However, calculations using the collaborative study data were performed to evaluate analytical bias. Calculations were performed according to ASTM Practice D2777.

13.2.1 In the collaborative study, the level of analytical accuracy for determining certain concentrations of silicon, sodium, strontium, chromium. and barium in the dilute acid solution standards (prepared using $60 / 40$ weight percent sulfuric/nitric acid solution) having a $\mathrm{pH}$ of $4.3 \pm 0.05$ was not as good as desired based on the criterion given in ASTM Practice D2777. For the levels of aluminum, boron, and calcium in these standards, the analytical 
accuracy was acceptable based on the 02777 criterion. The level of analytical accuracy for determining certain concentrations of calcium, silicon, zinc, barium, lead, and magnesium in the dilute acid solution standards (prepared using 60/40 weight percent sulfuric/nitric acid solution) having a $\mathrm{pH}$ of $5.0 \pm 0.05$ was not as good as desired based on the criterion given in ASTM Practice D2777. For the levels of manganese in these standards, the analytical accuracy was acceptable based on the same criterion. Data and additional information concerning these evaluations are available in Report PCN:33-000004-56.

13.3 Comparative data evaluation:

13.3.1 Calculations using the collaborative study data were performed to evaluate the effect of extracting the test materials using deionized, distilled water versus the acidic extraction fluids. Data and information concerning these evaluations are available in Report PCN:33-000004-56. Elemental solubility in sequential batch water extracts versus acid solution extracts depends on the solution chemistry of the extraction slurries. As a result, the effect of using an acidic extraction fluid versus water depends on the material being tested, the $\mathrm{pH}$ of the extraction fluid, the element or parameter(s) of interest, and the extraction number in the sequence of the sequential batch extraction. 
Table 1. Estimated Multiple-Laboratory Precision of the Sequential Batch Eutraction Method Using Acidic Extraction Fluid for the Spray Dryer waste, $\mu \mathrm{g} / \mathrm{g}$

\begin{tabular}{|c|c|c|c|c|c|c|c|c|}
\hline Extract 1 & $\underset{\text { num }}{\text { Alumi- }}$ & Barium & Boron & $\begin{array}{l}\text { Cal- } \\
\text { cium }\end{array}$ & $\begin{array}{l}\text { Chro- } \\
\text { mium }\end{array}$ & $\begin{array}{c}\text { sili- } \\
\text { con }\end{array}$ & Sodium & $\begin{array}{c}\text { stron- } \\
\text { tium }\end{array}$ \\
\hline $\begin{array}{l}\bar{x} \\
\bar{x}_{a} \\
\mathbf{s} \\
s_{a} \\
s_{e}\end{array}$ & $\begin{array}{l}25.5 \\
29 \\
3.2 \\
3 \\
1\end{array}$ & $\begin{array}{r}14 \\
7 \\
7 \\
4 \\
6\end{array}$ & $\begin{array}{l}1.8 \\
4 \\
0.4 \\
3 \\
----^{2}\end{array}$ & $\begin{array}{r}3.755 \\
3.927 \\
261 \\
182 \\
187\end{array}$ & $\begin{array}{l}2.8 \\
2.8 \\
0.3 \\
0.2 \\
0.2\end{array}$ & $\begin{array}{r}181 \\
171 \\
15 \\
9 \\
12\end{array}$ & $\begin{array}{r}3,515 \\
3,669 \\
342 \\
397 \\
--{ }^{-} a\end{array}$ & $\begin{array}{r}214 \\
188 \\
18 \\
10 \\
15\end{array}$ \\
\hline
\end{tabular}

Extract 3

\begin{tabular}{|c|c|c|c|c|c|c|c|}
\hline $\begin{array}{l}\bar{x} \\
\bar{x} \\
s_{a} \\
s_{a} \\
s_{e}\end{array}$ & $\begin{array}{r}108 \\
118 \\
10 \\
9 \\
4\end{array}$ & $\begin{array}{r}45 \\
50 \\
19 \\
4 \\
19\end{array}$ & $\begin{array}{l}2.3 \\
4 \\
0.9 \\
3 \\
--\infty\end{array}$ & $\begin{array}{r}2.795 \\
2.611 \\
111 \\
212 \\
---a\end{array}$ & $\begin{array}{r}106 \\
107 \\
8 \\
8 \\
0\end{array}$ & $\begin{array}{r}100 \\
116 \\
11 \\
28 \\
---2 a\end{array}$ & $\begin{array}{r}61.9 \\
46.7 \\
5.3 \\
2.7 \\
4.6\end{array}$ \\
\hline
\end{tabular}

Extract 5

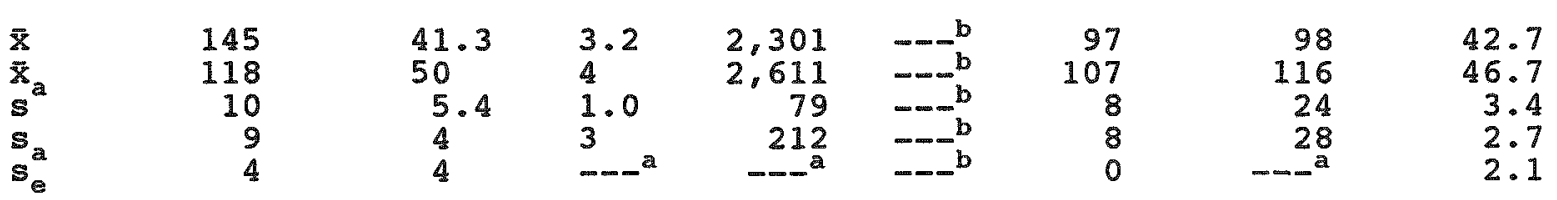

Extract 7

\begin{tabular}{|c|c|c|c|c|c|c|c|c|}
\hline $\begin{array}{l}\bar{x} \\
\bar{x}_{a} \\
s^{2} \\
s_{a} \\
\mathbf{s}_{e}\end{array}$ & $\begin{array}{r}164 \\
172 \\
14 \\
11 \\
9\end{array}$ & $\begin{array}{l}26.8 \\
31 \\
2.4 \\
5 \\
---^{a}\end{array}$ & $\begin{array}{l}4.5 \\
4 \\
1.0 \\
3 \\
---^{a}\end{array}$ & $\begin{array}{r}2,119 \\
1,983 \\
79 \\
149 \\
---^{a}\end{array}$ & $\begin{array}{l}--{ }_{-b}^{b} \\
---b \\
---b \\
---b \\
--b\end{array}$ & $\begin{array}{l}80.1 \\
75 \\
6.8 \\
6 \\
3\end{array}$ & $\begin{array}{r}50 \\
48 \\
11 \\
28 \\
--2 a\end{array}$ & $\begin{array}{l}26.8 \\
19 \\
1.7 \\
1 \\
1\end{array}$ \\
\hline
\end{tabular}

Extract 10

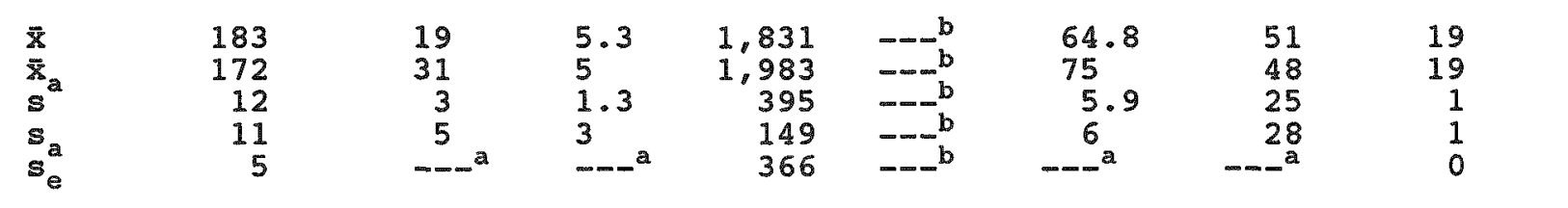

a The multiple-laboratory standard deviation of the extraction procedure can not be determined. See section 13.1.7.

b Values for extracts 3-10 can not be calculated because the data sets contain "less-than" values. 
Table 2. Estimated Muliple-Laboratory Precision of the sequential Batch Extraction Method Using Acidic Extraction Fluid for the Composite Mining Waste, $\mu \mathrm{g} / \mathrm{g}$

\begin{tabular}{|c|c|c|c|c|c|c|c|}
\hline Extract 1 & Barium & Calcium & Lead & Magnesium & Manganese & silicon & zinc \\
\hline $\begin{array}{l}\overline{\mathrm{x}} \\
\overline{\mathrm{x}} \\
\mathrm{s}^{\mathrm{a}} \\
\mathrm{s}_{\mathbf{a}} \\
\mathbf{s}_{\mathrm{e}}^{\mathrm{a}}\end{array}$ & $\begin{array}{l}1.9 \\
3 \\
0.3 \\
4 \\
-. .--^{a}\end{array}$ & $\begin{array}{r}1,815 \\
1,614 \\
238 \\
110 \\
211\end{array}$ & $\begin{array}{r}63 \\
91 \\
8 \\
4 \\
7\end{array}$ & $\begin{array}{r}107 \\
102 \\
12 \\
5 \\
11\end{array}$ & $\begin{array}{r}36.7 \\
39.9 \\
5.5 \\
1.7 \\
5.2\end{array}$ & $\begin{array}{r}55 \\
44 \\
21 \\
3 \\
21\end{array}$ & $\begin{array}{r}297 \\
312 \\
35 \\
11 \\
33\end{array}$ \\
\hline
\end{tabular}

Extract 3

\begin{tabular}{|c|c|c|c|c|c|c|c|}
\hline $\begin{array}{l}\bar{z}_{\mathbf{z}} \\
\overrightarrow{\mathbf{x}}_{\mathrm{a}} \\
\mathbf{s} \\
\mathbf{s}_{\mathrm{a}} \\
\mathbf{s}_{\mathrm{e}}\end{array}$ & $\begin{array}{l}2.3 \\
3 \\
0.3 \\
4 \\
----^{2}\end{array}$ & $\begin{array}{r}448 \\
622 \\
64 \\
42 \\
48\end{array}$ & $\begin{array}{l}--{ }^{b} \\
--{ }^{b} \\
--{ }^{b} \\
---^{b} \\
--^{b}\end{array}$ & $\begin{array}{r}12 \\
14 \\
2 \\
1 \\
2\end{array}$ & $\begin{array}{l}3.6 \\
6.1 \\
1.3 \\
0.3 \\
1.3\end{array}$ & $\begin{array}{r}35 \\
34 \\
6 \\
3 \\
5\end{array}$ & $\begin{array}{c}13 \\
3.6 \\
10 \\
4.8 \\
9\end{array}$ \\
\hline
\end{tabular}

Extract 5

\begin{tabular}{|c|c|c|c|c|c|}
\hline $\begin{array}{l}6 \\
7 \\
2 \\
5 \\
-\end{array}$ & $\begin{array}{r}268 \\
248 \\
16 \\
11 \\
12\end{array}$ & $\begin{array}{l}---^{b} \\
--^{b} \\
---^{b} \\
--^{b} \\
--^{b}\end{array}$ & $\begin{array}{r}10 \\
14 \\
2 \\
1 \\
2\end{array}$ & $\begin{array}{l}0.65 \\
1.0 \\
0.15 \\
0.1 \\
0.1\end{array}$ & $\begin{array}{r}20 \\
13 \\
3 \\
2 \\
2\end{array}$ \\
\hline
\end{tabular}

Extract 7

$\bar{x}$
$\bar{x}_{a}$
$\mathbf{s}$
$\mathbf{s}_{a}$
$\mathbf{s}_{e}$

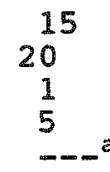

Extract 10

15
20
1
5
-
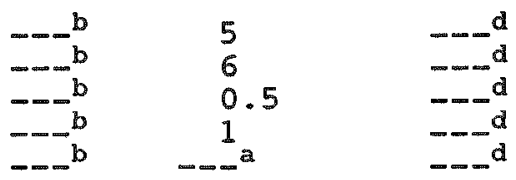

$\begin{array}{rr}14 & -\ldots c \\ 13 & -\cdots c \\ 2 & -\cdots c \\ 2 & -\cdots c \\ 0 & -\cdots c\end{array}$
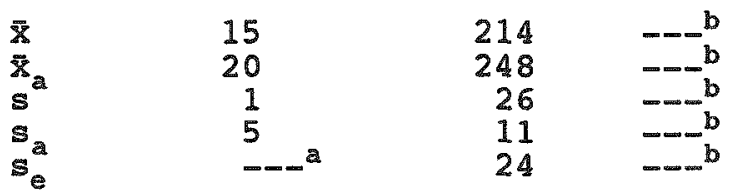

5
6
2
1
2

$---\frac{d}{d}$
$---\frac{d}{d}$
$-\infty-\infty$

$\begin{aligned} 14 & --{ }^{c} \\ 13 & --c \\ 3 & --c \\ 2 & ---^{c}\end{aligned}$

a The multiple-laboratory standard deviation of the extraction procedure can not be determined. See section 13.1.7.

b Values for extracts $3-10$ can not be calculated because the data sets contain "less-than" values.

c Values for extracts $4-10$ can not be calculated because the data sets contain "less-than" values.

d Values for extracts 6-10 can not be calculated because the data sets contain "less-than" values. 
Table 3. Estimated Single-operator precision of the sequential Batch Extraction Method Using Mcidic Extraction Fluid for the Spray Dryer Waste, $\mu \mathrm{g} / \mathrm{g}$

\begin{tabular}{|c|c|c|c|c|c|c|c|c|}
\hline Extract 1 & $\begin{array}{c}\text { Alumi- } \\
\text { num }\end{array}$ & Barium & Boron & $\begin{array}{l}\text { Cal- } \\
\text { cium }\end{array}$ & $\begin{array}{l}\text { Chro- } \\
\text { mium }\end{array}$ & $\begin{array}{c}\text { sili- } \\
\text { con }\end{array}$ & Sodium & $\begin{array}{l}\text { stron- } \\
\text { tium }\end{array}$ \\
\hline $\begin{array}{l}\bar{x} \\
\bar{x} \\
s_{0}^{a} \\
s_{o a}^{o} \\
s_{o e}\end{array}$ & $\begin{array}{l}25.5 \\
29 \\
1.8 \\
1 \\
1\end{array}$ & $\begin{array}{c}14 \\
7 \\
1 \\
0.1 \\
1\end{array}$ & $\begin{array}{l}1.8 \\
4 \\
0.3 \\
0.2 \\
0.2\end{array}$ & $\begin{array}{r}3,755 \\
3,927 \\
154 \\
120 \\
97\end{array}$ & $\begin{array}{l}2.8 \\
2.8 \\
0.2 \\
0.1 \\
0.2\end{array}$ & $\begin{array}{r}181 \\
171 \\
6 \\
8 \\
--^{a}\end{array}$ & $\begin{array}{r}3,515 \\
3,669 \\
50 \\
161 \\
---^{a}\end{array}$ & $\begin{array}{r}214 \\
188 \\
6 \\
3 \\
5\end{array}$ \\
\hline
\end{tabular}

Extract 3

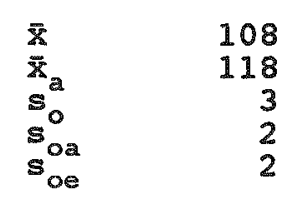

108
118
3
2
2

\begin{tabular}{|c|c|c|c|c|}
\hline $\begin{array}{r}45 \\
50 \\
2 \\
1 \\
2\end{array}$ & $\begin{array}{l}2.3 \\
4 \\
0.6 \\
0.2 \\
0.6\end{array}$ & $\begin{array}{r}2,795 \\
2,611 \\
55 \\
62 \\
-\quad-2\end{array}$ & 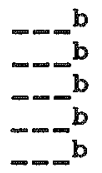 & $\begin{array}{r}106 \\
107 \\
2 \\
3 \\
--\infty\end{array}$ \\
\hline
\end{tabular}

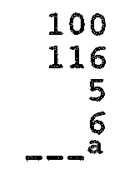

61.9

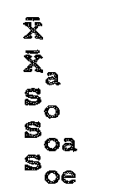

145
118
2
2
0

$41 \cdot 3$
50
1.2
$\frac{1}{1}$

$\begin{array}{lr}3.2 & 2,301 \\ 4 & 2,611 \\ 0.5 & 37 \\ 0.2 & 62 \\ 0.5 & --.\end{array}$

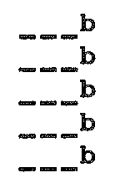

97
107
3
3
0

98
116
5
6
--2

42.7

46.7

$$
\begin{aligned}
& 1.5 \\
& 1 \\
& 1
\end{aligned}
$$

Extract 7

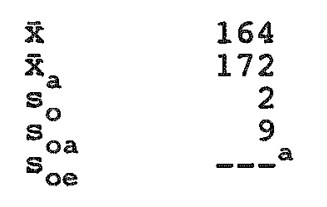

$$
\begin{array}{llrlc}
26.8 & 4.5 & 2,119 & -\ldots b & 80.1 \\
31 & 4 & 1,983 & --b & 75 \\
0.5 & 0.4 & 32 & --b & 2.6 \\
0.5 & 0.2 & 43 & --b & 3 \\
0 & 0.3 & ---\frac{b}{b} & --\infty b & ---^{a}
\end{array}
$$

50
48
2
3
--2

26.8

\begin{tabular}{|c|c|c|c|c|c|c|c|c|}
\hline $\begin{array}{l}\bar{x} \\
\bar{x} \\
\mathbf{s}_{a} \\
\mathbf{s}_{o a}^{0} \\
\mathbf{s}_{\text {oe }}\end{array}$ & $\begin{array}{r}183 \\
172 \\
2 \\
9 \\
---^{a}\end{array}$ & $\begin{array}{l}19 \\
31 \\
1 \\
0.5 \\
1\end{array}$ & $\begin{array}{l}5.3 \\
5 \\
0.5 \\
0.3 \\
0.4\end{array}$ & $\begin{array}{r}1,831 \\
1,983 \\
185 \\
43 \\
180\end{array}$ & $\begin{array}{l}-b b \\
---b \\
---b \\
--b \\
--b\end{array}$ & $\begin{array}{l}64.8 \\
75 \\
3.5 \\
3 \\
2\end{array}$ & $\begin{array}{r}51 \\
48 \\
4 \\
3 \\
3\end{array}$ & $\begin{array}{l}19 \\
19 \\
1 \\
0.2 \\
1\end{array}$ \\
\hline
\end{tabular}

Extract 10

a The single-operator standard deviation of the extraction procedure can not be determined. See section 13.1.7.

b Values for extracts $3-10$ can not be calculated because the data sets contain "less-than" values. 
Table 4. Estimated single-operator precision of the sequential Batch Extraction Method Using Acidic Extraction Fluid for the composite Mining Waste, $\mu \mathrm{g} / \mathrm{g}$

\begin{tabular}{|c|c|c|c|c|c|c|c|}
\hline Extract 1 & Barium & Calcium & Lead & Magnesium & Manganese & silicon & zinc \\
\hline $\begin{array}{l}\bar{x} \\
\bar{x}_{a} \\
s_{0}^{a} \\
s_{o a} \\
s_{o e}\end{array}$ & $\begin{array}{l}1.9 \\
3 \\
0.2 \\
0.2 \\
0\end{array}$ & $\begin{array}{r}1,815 \\
1,614 \\
139 \\
33 \\
135\end{array}$ & $\begin{array}{r}63 \\
91 \\
3 \\
3 \\
0\end{array}$ & $\begin{array}{r}107 \\
102 \\
7 \\
1 \\
7\end{array}$ & $\begin{array}{r}36.7 \\
39.9 \\
1.1 \\
0.5 \\
1.0\end{array}$ & $\begin{array}{r}55 \\
44 \\
2 \\
1 \\
2\end{array}$ & $\begin{array}{r}297 \\
312 \\
31 \\
6 \\
30\end{array}$ \\
\hline
\end{tabular}

Extract 3

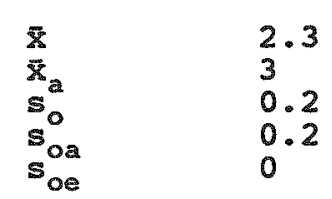

$\begin{array}{rll}448 & ---_{b}^{b} & 12 \\ 622 & ---b & 14 \\ 22 & ---b & 1 \\ 9 & ---b & 0.4 \\ 20 & ---b & 1\end{array}$

3.6
6.1
0.3
0.2
0.2

35
34
3
1
3

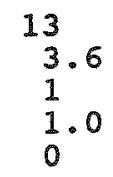

Extract 5

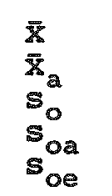

$$
\begin{aligned}
& 6 \\
& 7 \\
& 1 \\
& 0.05 \\
& 1
\end{aligned}
$$

$\begin{aligned} 268 & --b \\ 248 & --b \\ 14 & ---b \\ 5 & --b \\ 13 & ---b\end{aligned}$

10
14
1
0.4
1

$$
\begin{aligned}
& 0.65 \\
& 1.0 \\
& 0.12 \\
& 0.03
\end{aligned}
$$

Extract 7
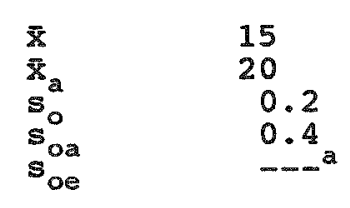

$\begin{aligned} 228 & --b \\ 248 & --b \\ 9 & --b \\ 5 & ---b \\ 7 & --b\end{aligned}$

5
6
0.3
0.2
0.2

\begin{tabular}{|c|c|c|c|c|c|c|c|}
\hline $\begin{array}{l}\overline{\bar{x}_{0}} \\
\bar{x}_{a} \\
\mathbf{s}_{0} \\
\mathbf{s}_{02} \\
\mathbf{s}_{00}\end{array}$ & $\begin{array}{l}15 \\
20 \\
1 \\
0.4 \\
1\end{array}$ & $\begin{array}{r}214 \\
248 \\
12 \\
5 \\
11\end{array}$ & $\begin{array}{l}-b b \\
---b \\
--b \\
--b \\
---b \\
=--b\end{array}$ & $\begin{array}{l}5 \\
6 \\
0.3 \\
0.2 \\
0.2\end{array}$ & $\begin{array}{l}---^{d} d \\
---d \\
---d \\
---d \\
---^{d} d\end{array}$ & $\begin{array}{r}14 \\
13 \\
1 \\
1 \\
0\end{array}$ & $\begin{array}{l}--^{c} \\
--{ }^{c} \\
--{ }^{c} \\
--c \\
--{ }^{c}\end{array}$ \\
\hline
\end{tabular}

$-m-d$
$-\infty-\frac{d}{d}$
$---\frac{d}{d}$
$---d$

20
13
2
1
2

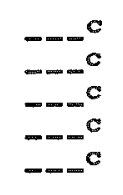

Extract 10

a The single-operator standard deviation of the extraction procedure can not be determined. See section 13.1 .7 .

b values for extracts $3-10$ can not be calculated because the data sets contain "less-than" values.

C Values for extracts $4-10$ can not be calculated because the data sets contain "less-than" values.

d Values for extracts $6-10$ can not be calculated because the data sets contain "less-than" values. 


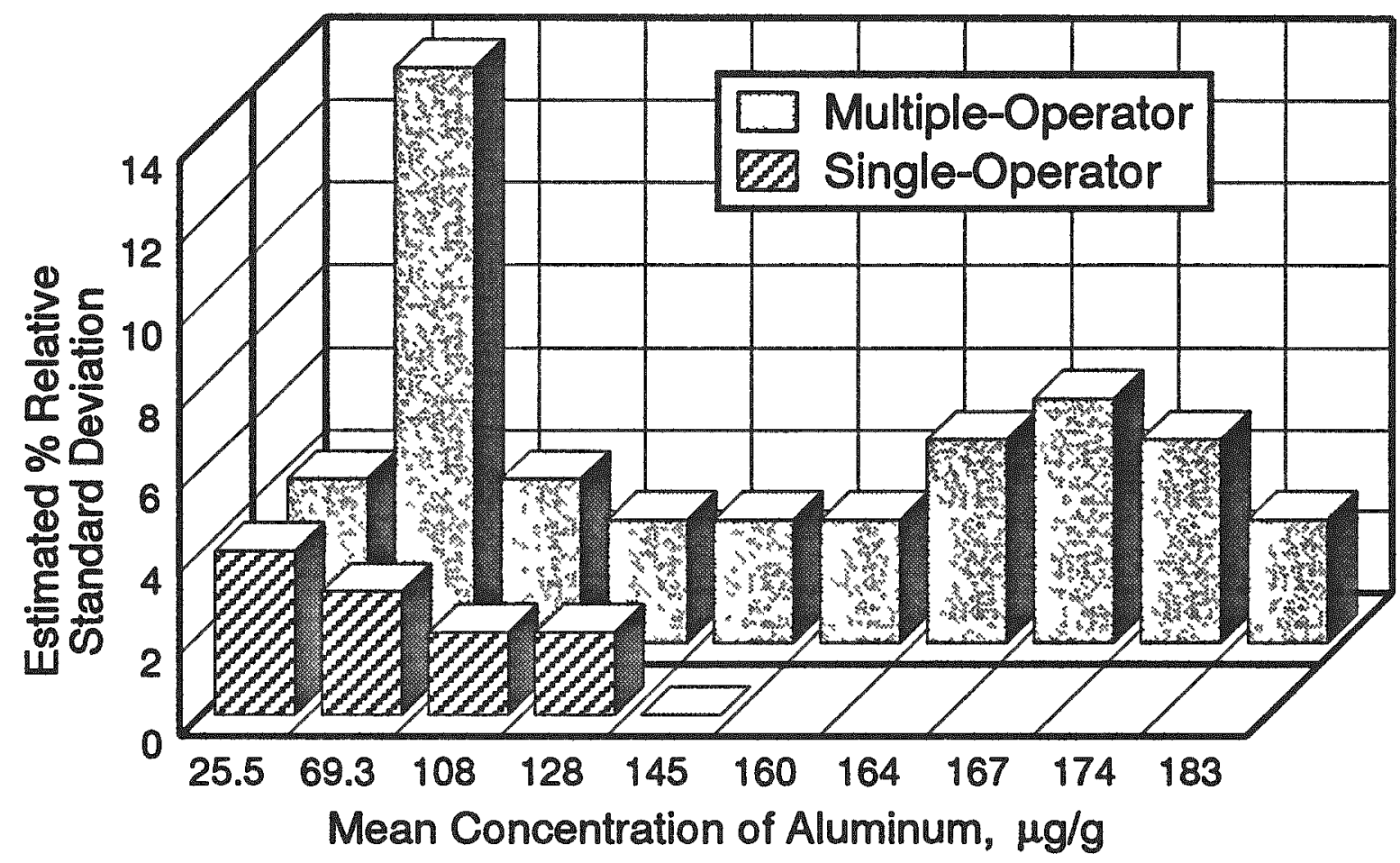

Figure 1. Estimated Precision of the Sequential Batch Extraction Method for Aluminum in the Spray Dryer Waste

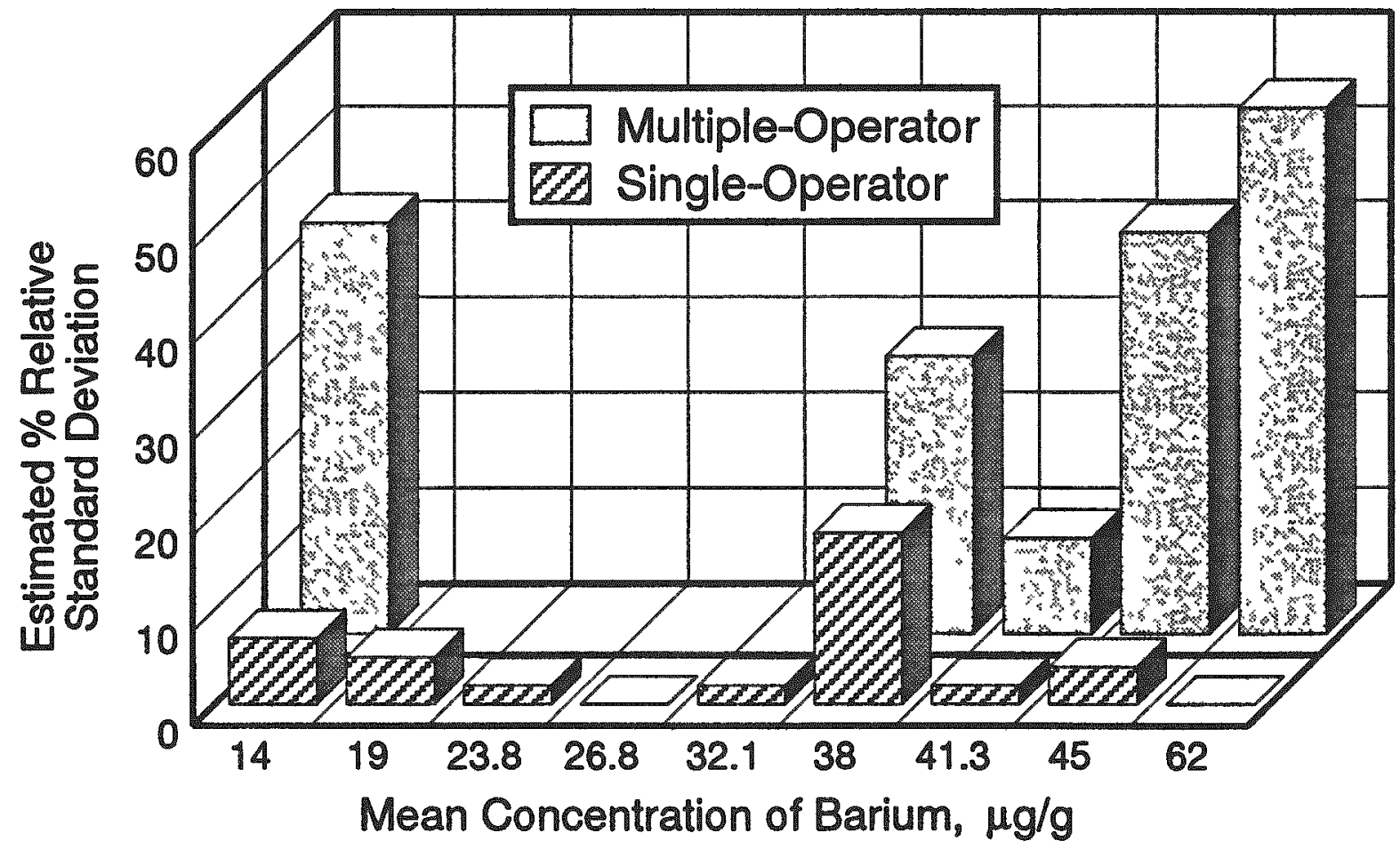

Figure 2. Estimated Precision of the Sequential Batch Extraction Method for Barium in the Spray Dryer Waste 


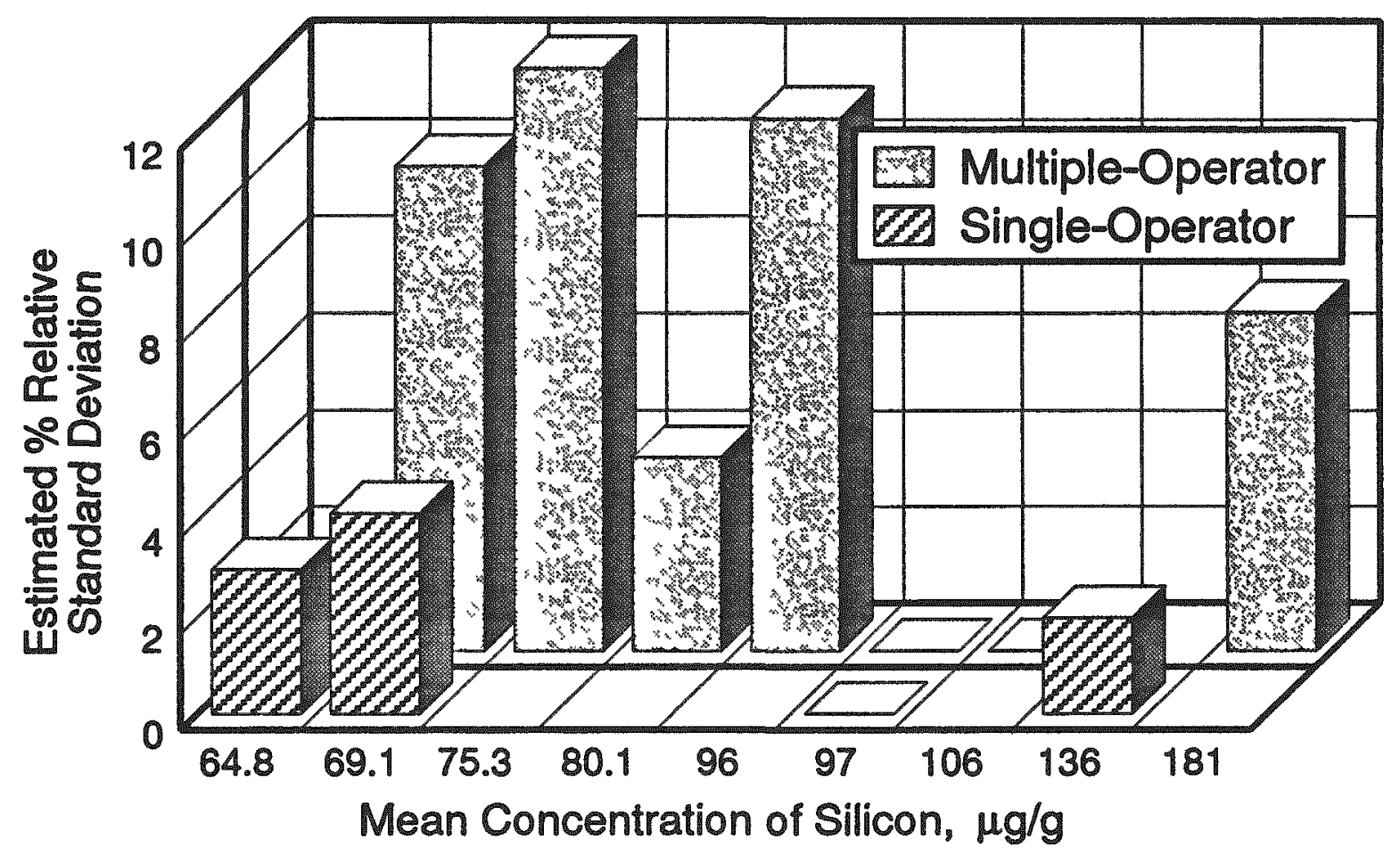

Figure 3. Estimated Precision of the Sequential Batch Extraction Method for Silicon in the Spray Dryer Waste

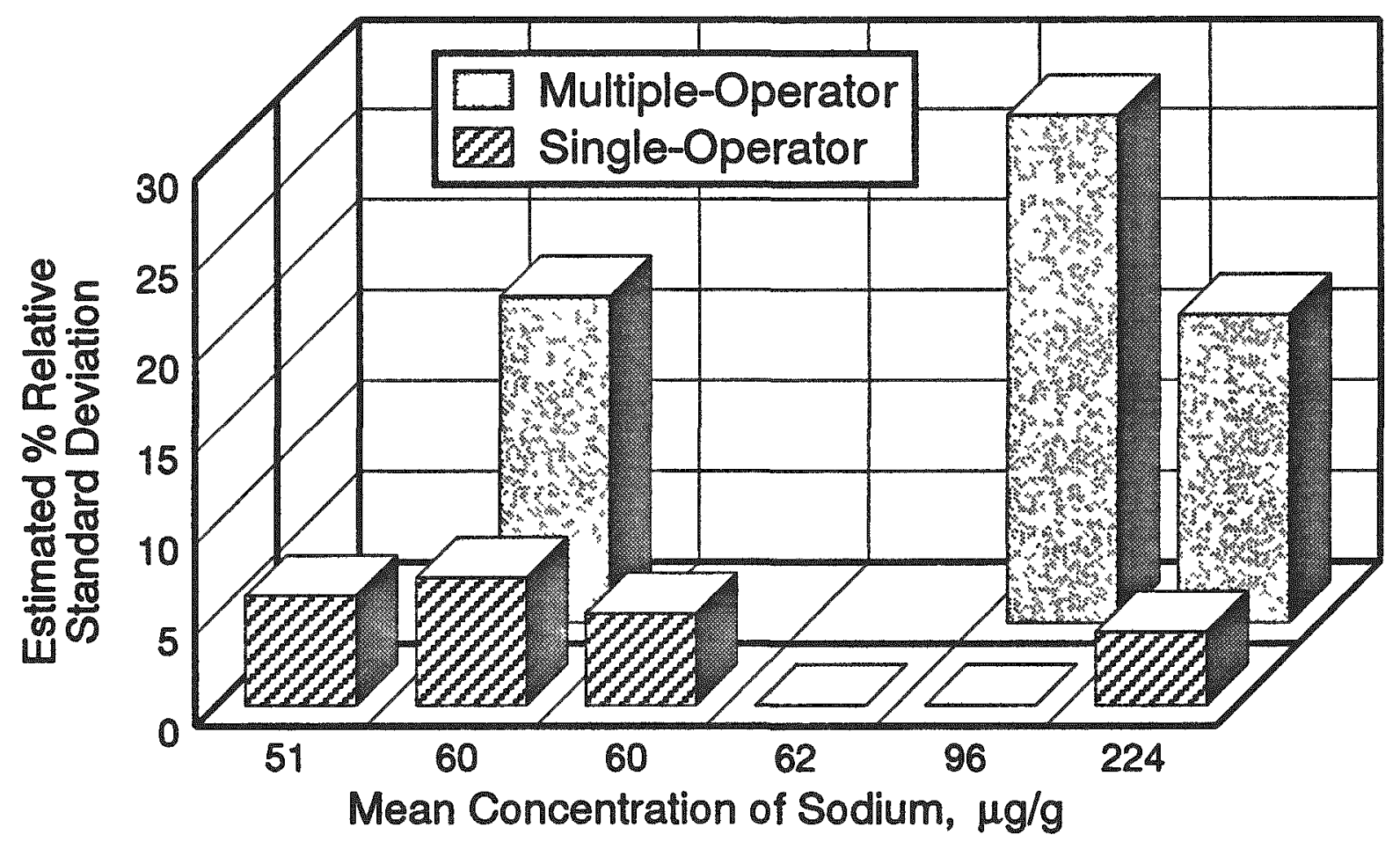

Figure 4. Estimated Precision of the Sequential Batch Extraction Method for Sodium in the Spray Dryer Waste 


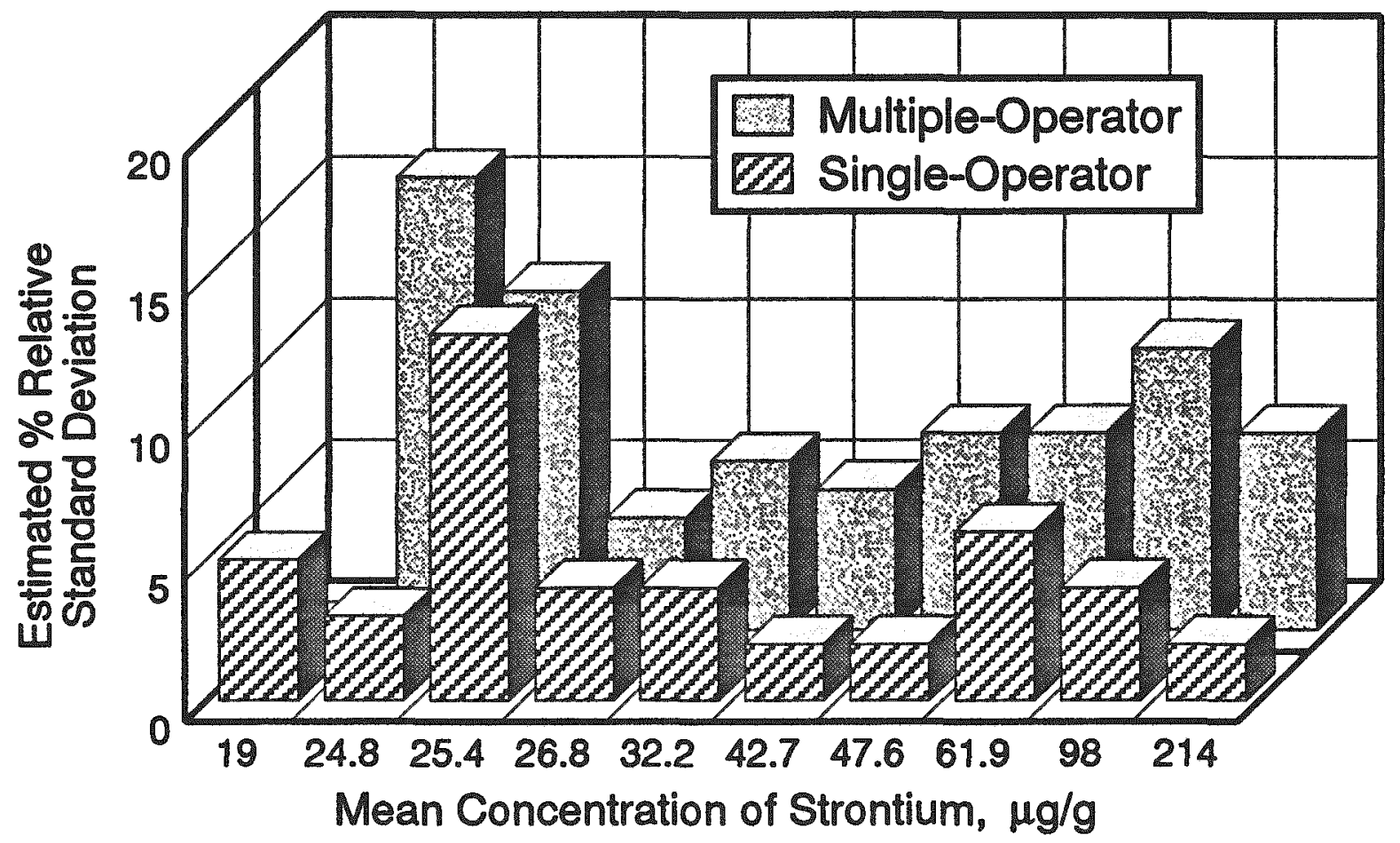

Figure 5. Estimated Precision of the Sequential Batch Extraction Method for Strontium in the Spray Dryer Waste

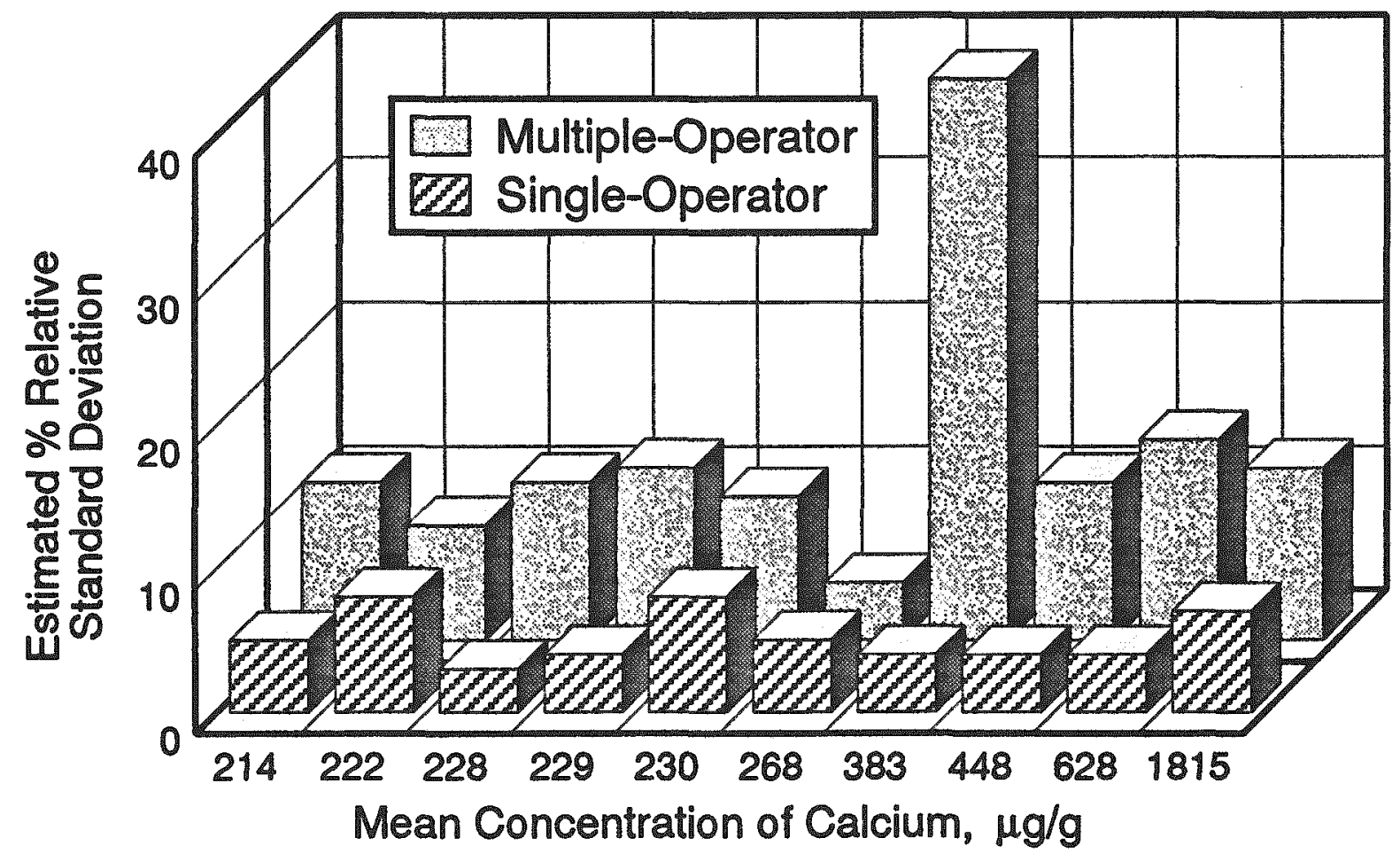

Figure 6. Estimated Precision of the Sequential Batch Extraction Method for Calcium in the Composite Mining Waste 


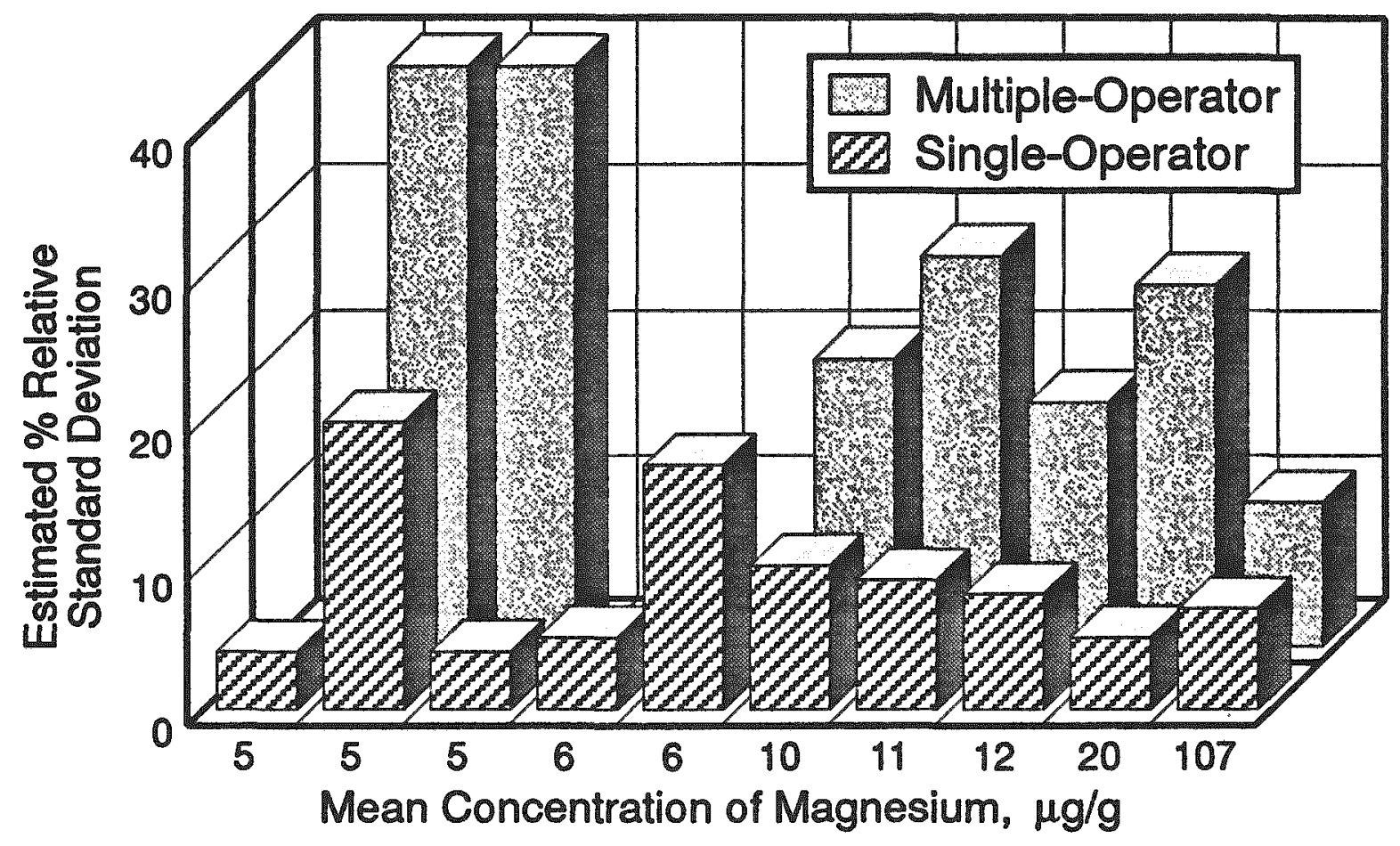

Figure 7. Estimated Precision of the Sequential Batch Extraction Method for Magnesium in the Composite Mining Waste

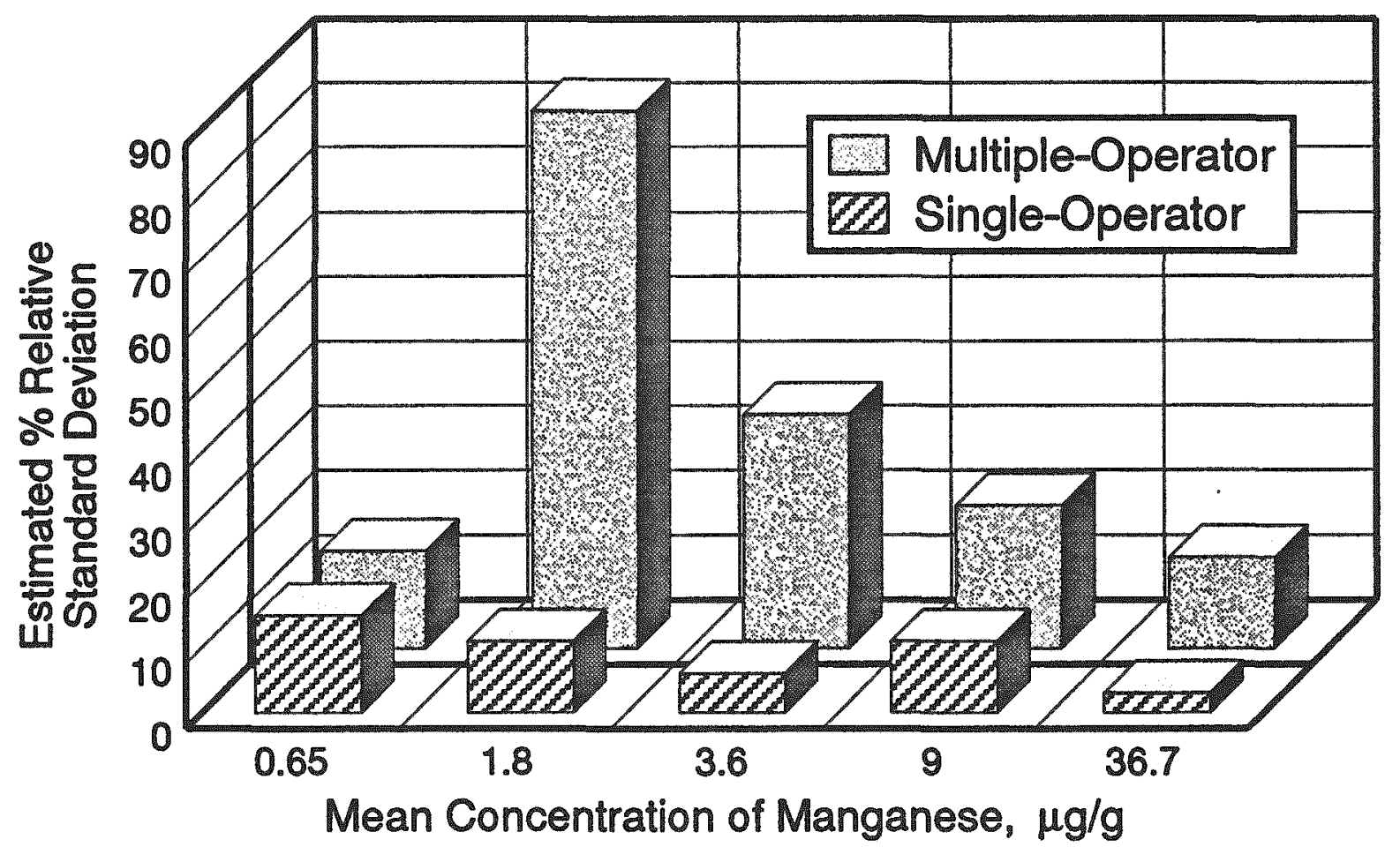

Figure 8. Estimated Precision of the Sequential Batch Extraction Method for Manganese in the Composite Mining Waste 


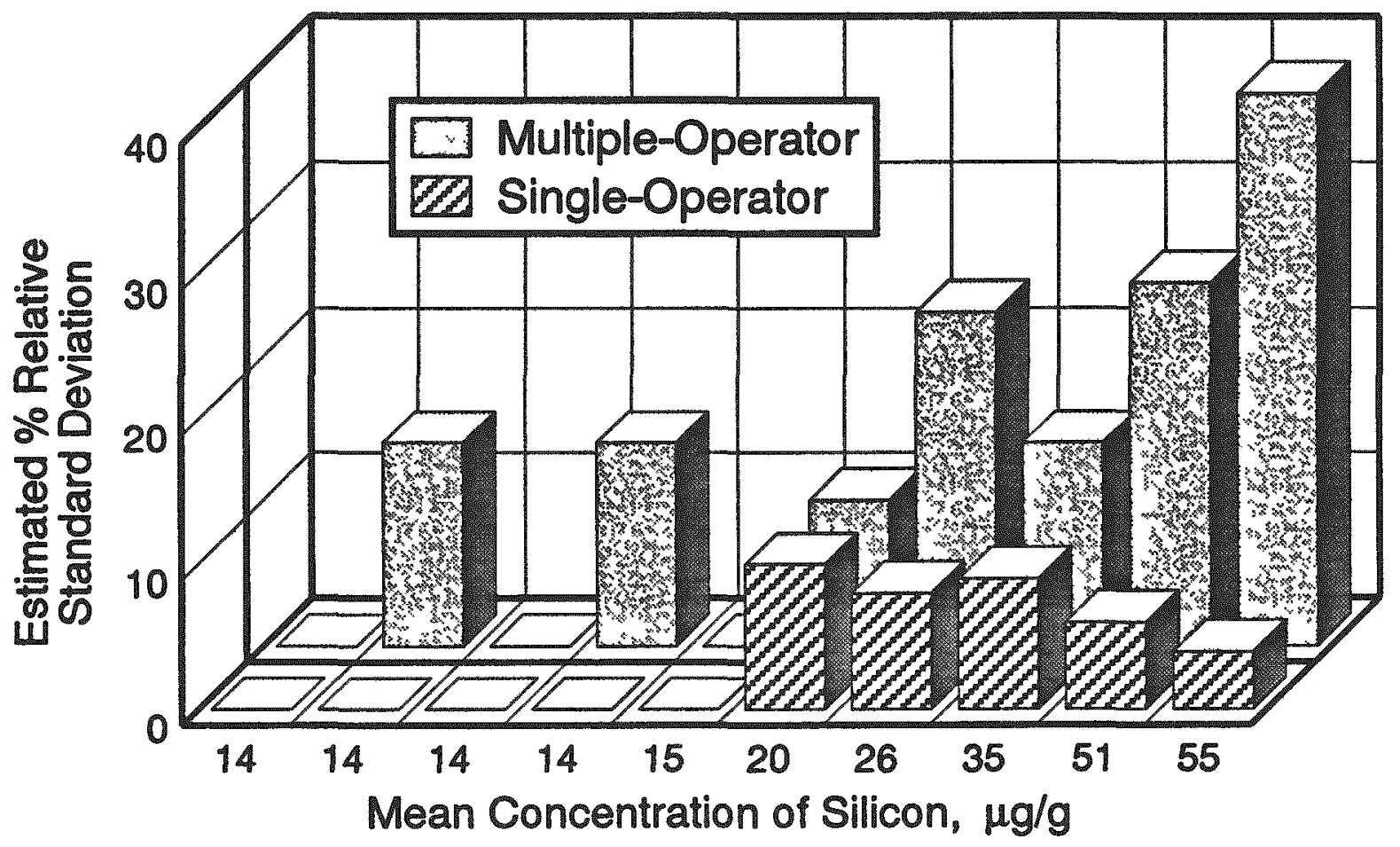

Figure 9. Estimated Precision of the Sequential Batch Extraction Method for Silicon in the Composite Mining Waste 


This cover slock is $30 \%$ post-consumer waste and $30 \%$ pre-consumer waste, and is recyclable. 